\title{
OPTIMAL CONTROL OF ATMOSPHERIC AIR INJECTION FOR ENHANCED OIL RECOVERY
}

\author{
By
}

\begin{abstract}
Shahrad Khodaei Booran
BEng in Chemical Engineering Co-op, Ryerson University, Toronto, 2011

MASc in Chemical Engineering, Ryerson University, Toronto, 2013
\end{abstract}

A dissertation presented to

Ryerson University

\author{
in partial fulfillment of the \\ requirements for the degree of \\ Doctor of Philosophy
}

in the program of

Chemical Engineering

Toronto, Ontario, Canada, 2017

(O)Shahrad Khodaei Booran, 2017 


\section{Author's Declaration}

I hereby declare that I am the sole author of this dissertation. This is a true copy of the dissertation, including any required final revisions, as accepted by my examiners.

I authorize Ryerson University to lend this dissertation to other institutions or individuals for the purpose of scholarly research.

I further authorize Ryerson University to reproduce this dissertation by photocopying or by other means, in total or in part, at the request of other institutions or individuals for the purpose of scholarly research.

I understand that my dissertation may be made electronically available to the public. 


\title{
OPTIMAL CONTROL OF ATMOSPHERIC AIR INJECTION FOR ENHANCED OIL RECOVERY \\ Doctor of Philosophy
}

2017

Shahrad Khodaei Booran

Chemical Engineering

Ryerson University

\begin{abstract}
Gas-based enhanced oil recovery (EOR) processes rely on the injection of gases such as carbon dioxide, nitrogen, and natural gas into heavy oil reservoirs to reduce inherent oil viscosity. Although these processes are very promising, they face the problem of limited and costly gas supply.
\end{abstract}

This study investigates the conditions, specifically temperature variation, under which freely available air at low temperatures, low pressures, and non-reactive environments for heavy oil recovery. To that end, preliminary experiments are carried out to demonstrate the possibility of beneficial effects of air temperature variation with time. Furthermore, this research aims to utilize the theory of optimal control to determine optimal air temperature versus time function to maximize the heavy oil recovery. For this purpose, the conditions necessary for optimal control are derived and utilized in a computational algorithm. 
The preliminary experiments are executed by injecting air into a lab-scale heavy oil reservoir at different pressures (0.169-0.514 $\mathrm{MPa}$ absolute) and temperatures in the range of $25-90^{\circ} \mathrm{C}$. Reservoirs of four different permeabilities (40-427 Darcy) are used in experiments. When air is injected with a periodic temperature variation between $90^{\circ} \mathrm{C}$ and $75^{\circ} \mathrm{C}$ that has an average of $78^{\circ} \mathrm{C}$, the recovery is increased from $58.2 \%$ to $69.1 \%$ of the original-oil-in-place (OOIP) in comparison to that using constant temperature air injection at the maximum temperature of $90^{\circ} \mathrm{C}$. That is a considerable improvement of oil recovery by $18.6 \%$.

Furthermore, utilizing optimal control the optimal interfacial temperature versus time (control policy) is determined between $90^{\circ} \mathrm{C}$ and $82^{\circ} \mathrm{C}$, which registers $20.66 \%$ increase in the oil recovery in comparison to that at the constant temperature of $90^{\circ} \mathrm{C}$. The accuracy of optimal control is experimentally validated. The results show that the average relative difference between the predicted heavy oil recovery and the experimental value is a low value of $1.82 \%$. 


\section{Acknowledgements}

I wish to express my sincere gratitude to Dr. Simant R. Upreti, and Dr. Farhad Ein-Mozaffari for their kind guidance, supervision, and suggestions throughout the entire period of my studies.

I am very grateful for the invaluable assistance of faculty members and technologists in the Chemical Engineering Department of Ryerson University, especially those provided by Ali Hemmati, Daniel Boothe, and Tondar Tajrobehkar during the equipment manufacture and system setup.

I would also like to thank Goretti Praticante and Louise Lichacz in the Department of Chemical Engineering. 


\section{Dedication}

Dedicated to my mother for her love and support throughout my life. 


\section{Table of Contents}

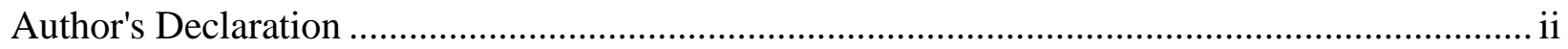

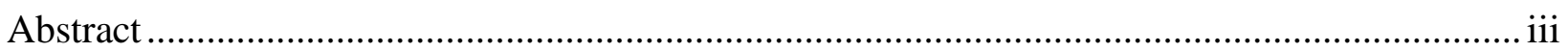

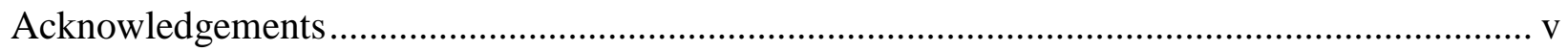

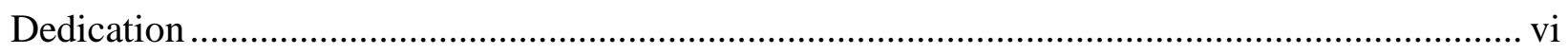

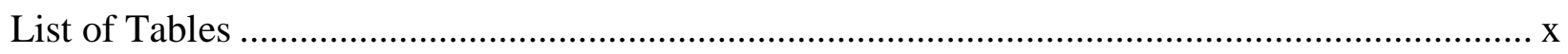

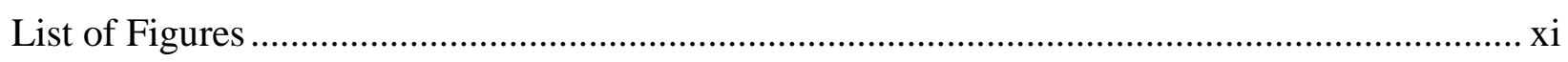

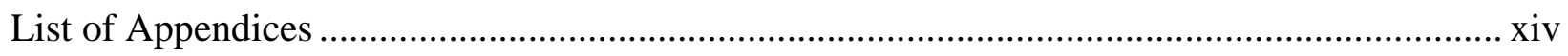

List of Symbols and Nomenclature .............................................................................

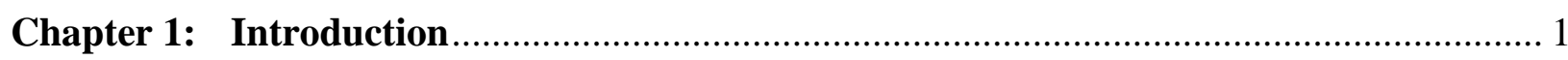

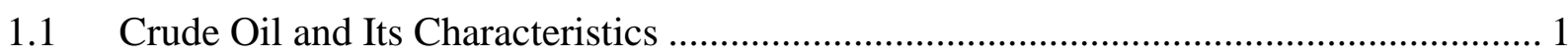

1.2 Crude Oil and Energy Source................................................................................ 2

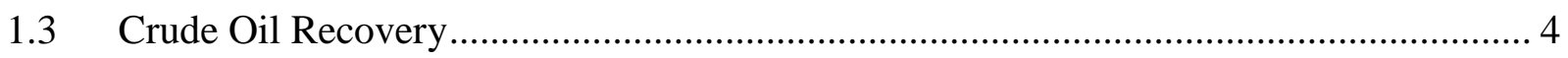

1.4 Heavy Oil and Bitumen and Enhanced Oil Recovery ............................................. 5

1.5 Challenges and Research Objectives.................................................................... 9

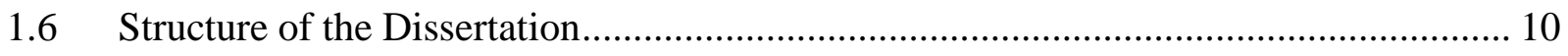

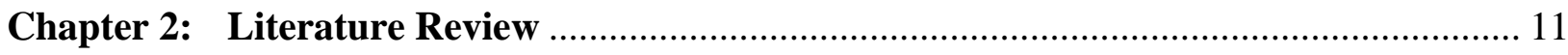

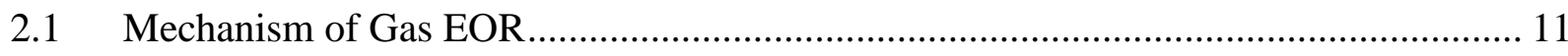

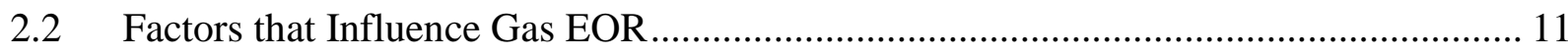

2.2.1 Heavy Oil Viscosity and Gas EOR ............................................................. 12

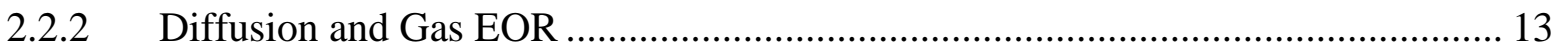

2.2.3 Dispersion and Gas EOR ............................................................................. 15

2.2.4 Permeability of the Reservoir and Gas EOR ................................................... 15

2.3 Optimal Control and Enhanced Oil Recovery...................................................... 16

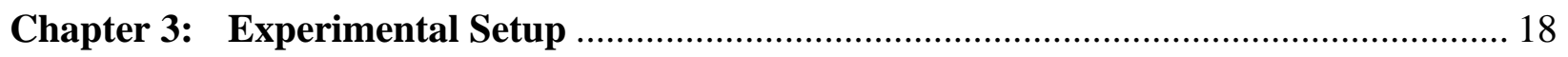

3.1 Air Injection Experimental Setup...................................................................... 18

3.2 Experimental Procedure ....................................................................................... 22

3.3 Physical Model Preparation ..................................................................................... 24

3.3.1 Permeability of Physical Model..................................................................... 25

3.4 Solvent Gas Solubility and Live Oil Density ....................................................... 26

Interfacial Solvent Concentration Setup ............................................................. 27

3.5.1 Experimental Procedure and Interfacial Solvent Concentration.......................... 28

3.6 Experimental Results Repeatability ................................................................... 30 


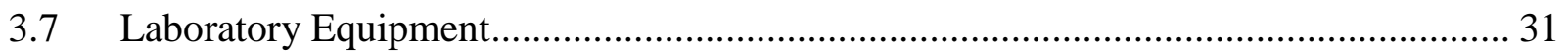

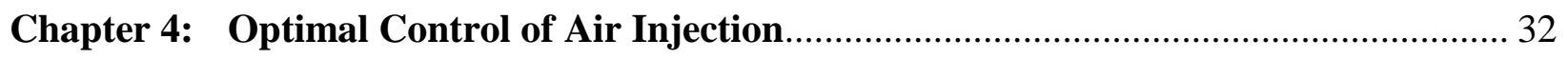

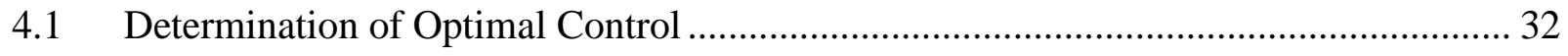

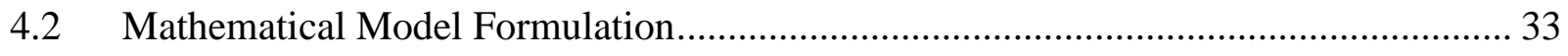

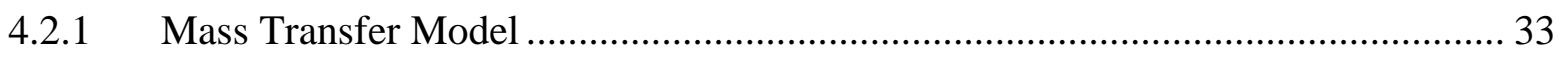

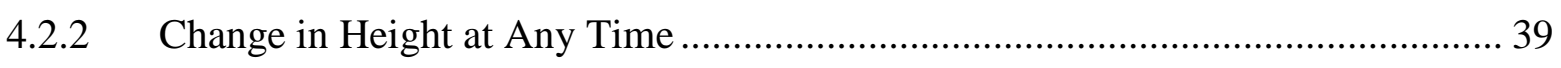

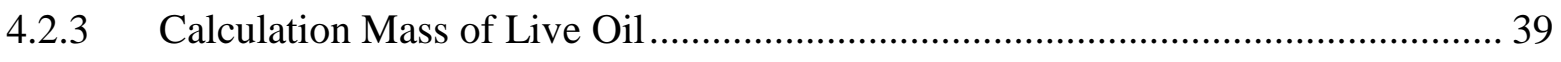

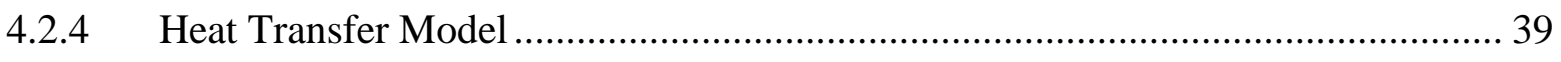

$4.3 \quad$ Formulation of Optimal Control Problem................................................................... 42

4.4 Necessary Conditions for the Optimal Control ……………...................................... 45

4.5 Implementation of the Improvement Method ........................................................... 57

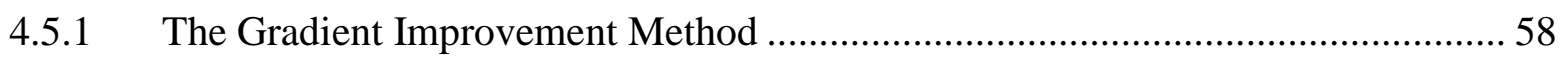

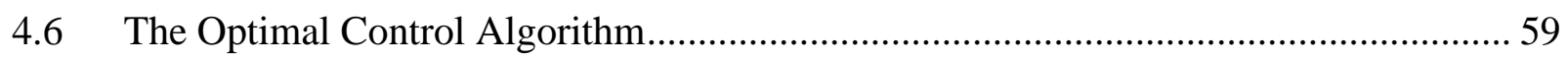

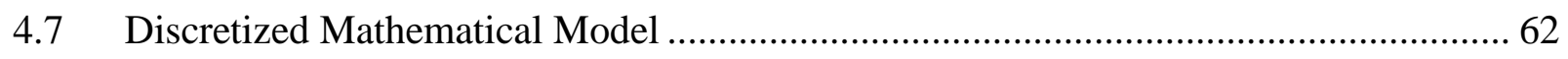

4.7.1 Finite Difference for conversion of PDE to ODE for Heat Transfer ........................ 62

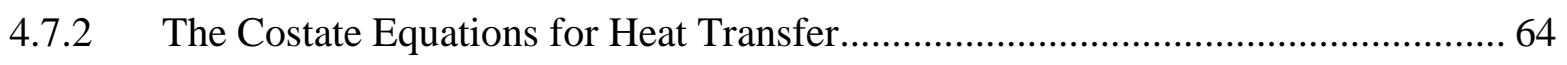

4.7.3 Finite Difference for conversion of PDE to ODE for Mass Transfer ...................... 66

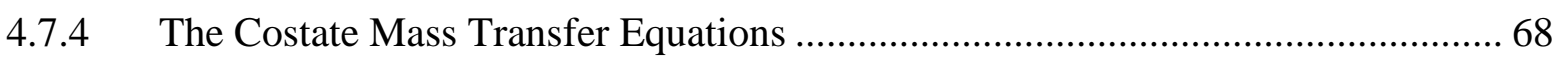

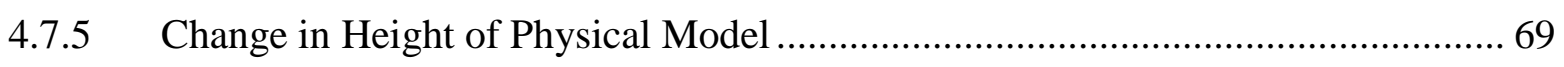

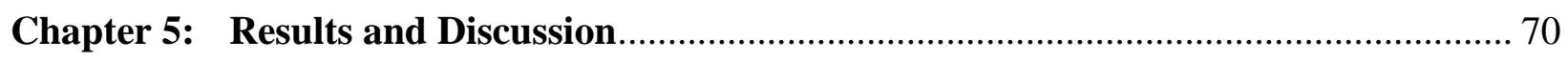

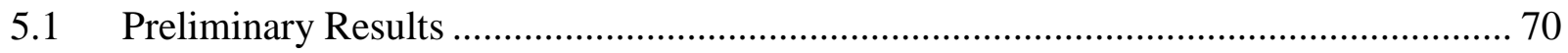

5.1.1 Effect of Permeability on Heavy Oil Recovery ................................................... 70

5.1.2 Effect of Pressure on Heavy Oil Recovery ………………….............................. 73

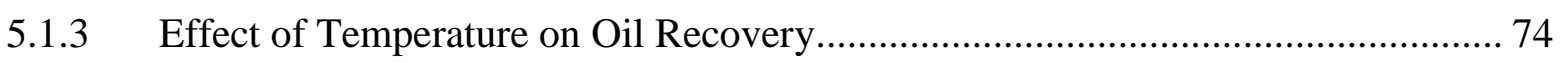

5.1.3.1 Effect of Air Temperature in Isothermal Experiments......................................... 74

5.1.3.2 Effect of Variation in the Air Temperature ……............................................... 78

5.1.4 Effect of Varying Temperature with Different Permeabilities ............................... 81

5.1.5 Absence of Chemical Reactions .......................................................................... 85

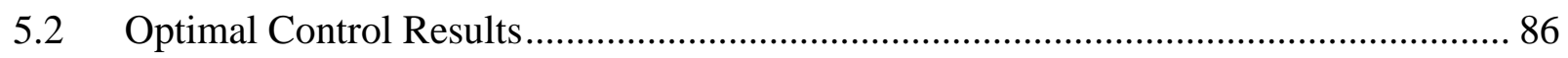

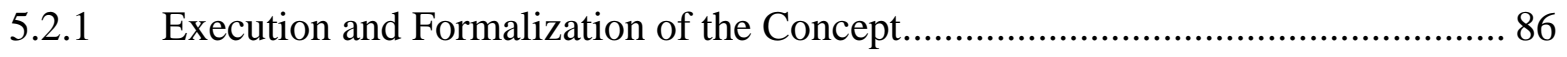

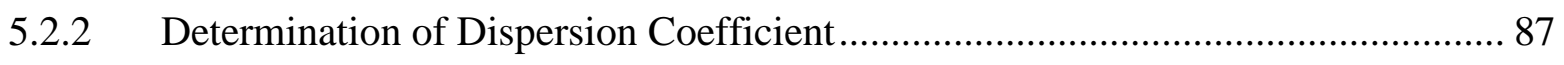

5.2.3 Optimal Control Policy for Air Interfacial Temperature ………………............... 92

5.2.4 Validation of Optimal Control Policy .............................................................. 100

5.2.5 Enhancement of Heavy Oil Recovery ................................................................. 101 
5.2.6 Permeability Effect on Optimal Policy ........................................................ 102

5.2.7 Sensitivity Analyses of the Model Parameters ................................................. 107

5.2.8 Potential for Field Scale .................................................................................. 110

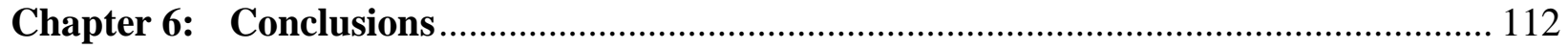

Appendix A: Porous Medium Permeability Calculation .............................................. 114

Appendix B: Solubility and Live Oil Density ........................................................... 115

Appendix C: Preliminary Experimental Results for Nitrogen Injection ......................... 117

Appendix D: Dispersion Coefficient Calculation for Nitrogen Injection ........................ 126

Appendix E: Optimal Control Policy for Nitrogen Interfacial Temperature ................... 128

Appendix F: Permeability Effect on Optimal Policy for Nitrogen Injection ................... 132

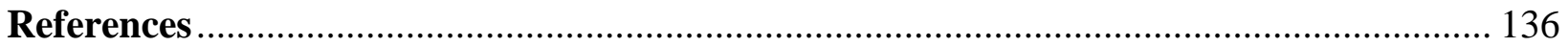




\section{List of Tables}

Table 1: Classification of crude oil according to the API degree ........................................... 2

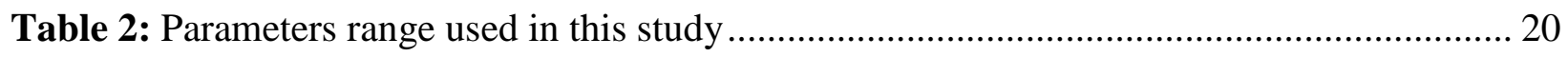

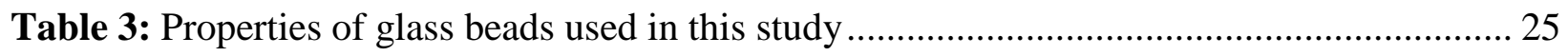

Table 4: Parameters for calculation of permeability for glass bead size 0.248-0.210 mm ......... 26

Table 5: Temperature versus interfacial concentration for air at pressure of $0.514 \mathrm{MPa}$........... 29

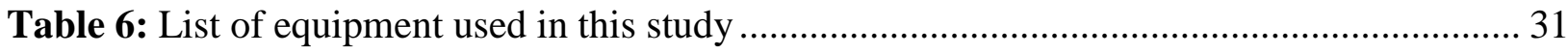

Table 7: Dissolved air in heavy oil at constant temperature $\left(25^{\circ} \mathrm{C}\right)$ and different pressures ....... 74

Table 8: Live oil viscosity and dissolved air fraction in oil at constant pressure of $0.514 \mathrm{MPa}$ and

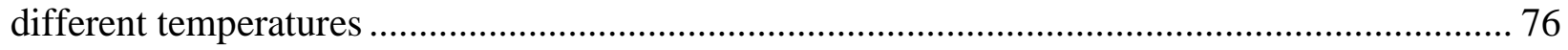

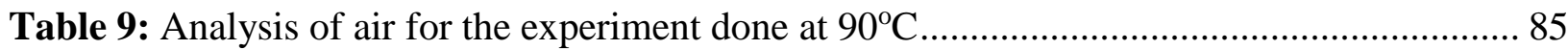

Table 10: Simulation model parameters of the mathematical model (Pressure 0.514 MPa, 204

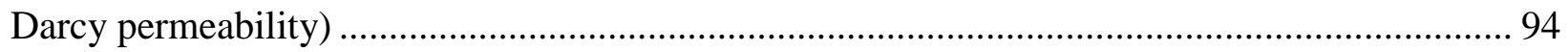

Table 11: Comparison of maximum objective functional obtained from different initial interfacial

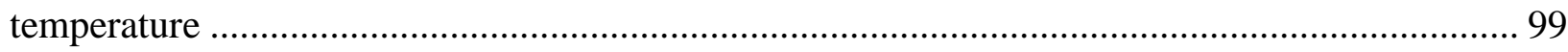

Table 12: Change in maximum objective functional by variation in parameters ................... 109

Table 13: Oil recoveries obtained in previous studies with different solvents ....................... 111

Table 14: Calculated air solubility and live oil density at $25^{\circ} \mathrm{C}$ and 204 Darcy .................... 115

Table 15: The result of live oil viscosity, live oil density, and air solubility at different temperatures 


\section{List of Figures}

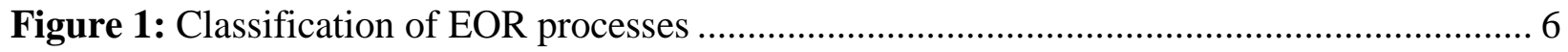

Figure 2: Schematic diagram of the experimental setup. The list of equipment in order are: 1-gas flow meter, 2-valve, 3-load cell, 4- pressure valve, 5- physical model, 6- thermometer, 7- funnel, 8-collection tube, 9- viscometer, 10- flash tank, and 11-water columns ............................... 21

Figure 3: Picture of Air Injection Experimental Set .......................................................... 23

Figure 4:Schematic of solvent-heavy oil system for the measurement of interfacial solvent

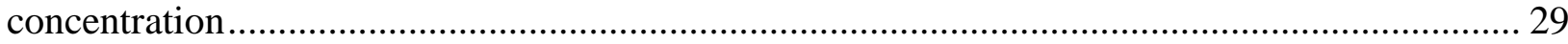

Figure 5: Cylindrical Model and Node Distribution......................................................... 38

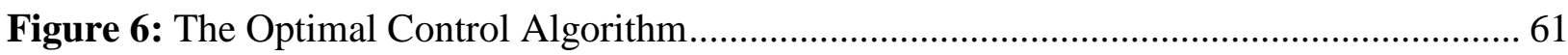

Figure 7: Oil recovery of air injection versus time for different permeabilities at $0.514 \mathrm{MPa}$

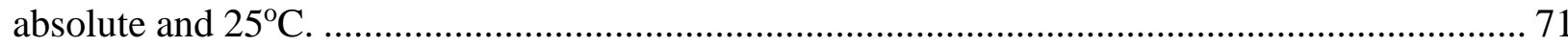

Figure 8: Variation of production rate of air injection with model permeability at $0.514 \mathrm{MPa}$

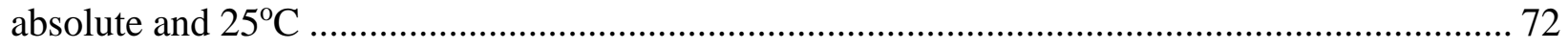

Figure 9: Oil recovery versus time at $25^{\circ} \mathrm{C}$ air temperature, 204 Darcy model permeability, and

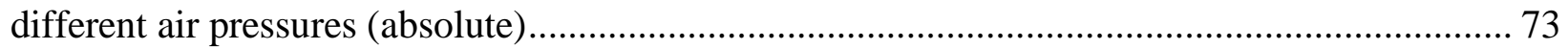

Figure 10: Oil recovery versus time at $0.514 \mathrm{MPa}$ absolute air pressure, 204 Darcy model

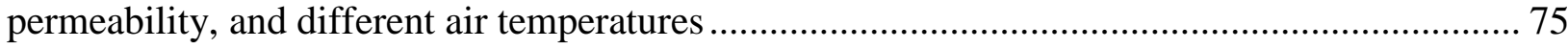

Figure 11: Correlation between viscosity and dissolved air mass fraction at $0.514 \mathrm{MPa}$ absolute and 204 Darcy permeability of the physical model ....................................................... 77

Figure 12: Oil recovery versus time at $0.514 \mathrm{MPa}$ absolute air pressure, 204 Darcy model permeability, and constant $\left(90^{\circ} \mathrm{C}\right)$ as well as periodically varying air temperature in the range, 50$90^{\circ} \mathrm{C}$.

Figure 13: Oil recovery versus time at $0.514 \mathrm{MPa}$ absolute air pressure, 204 Darcy model permeability, and constant $\left(90^{\circ} \mathrm{C}\right)$ as well as periodically varying air temperature in the range, $75-90^{\circ} \mathrm{C}$

Figure 14: Oil recovery versus time at $0.514 \mathrm{MPa}$ absolute air pressure, constant $\left(90^{\circ} \mathrm{C}\right)$ as well as periodically varying air temperature in the range, $75-90^{\circ} \mathrm{C}$, and 40 Darcy model permeability

Figure 15: Oil recovery versus time at $0.514 \mathrm{MPa}$ absolute air pressure, constant $\left(90^{\circ} \mathrm{C}\right)$ as well as periodically varying air temperature in the range, $75-90^{\circ} \mathrm{C}$, and 87 Darcy model permeability

Figure 16: Oil recovery versus time at $0.514 \mathrm{MPa}$ absolute air pressure, constant $\left(90^{\circ} \mathrm{C}\right)$ as well as periodically varying air temperature in the range, $75-90^{\circ} \mathrm{C}$, and 427 Darcy model permeability 
Figure 17: Dispersion coefficient function of air injection in heavy oil (Pressure 0.514 MPa;

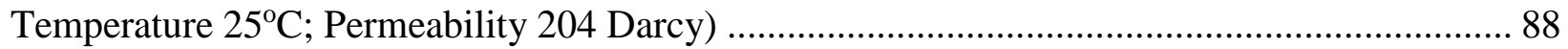

Figure 18: Objective functional versus iteration number for dispersion coefficient at air pressure $0.514 \mathrm{MPa}$, temperature $25^{\circ} \mathrm{C}$, and permeability 204 Darcy 90

Figure 19: Dispersion coefficient function of air injection in heavy oil at different temperatures $\left(25^{\circ} \mathrm{C}, 50^{\circ} \mathrm{C}, 75^{\circ} \mathrm{C}\right.$, and $90^{\circ} \mathrm{C}$ ) (Pressure $0.514 \mathrm{MPa}$; Permeability 204 Darcy) ....................... 91

Figure 20: Maximum dispersion coefficient of air at different temperatures ........................... 92

Figure 21: The interfacial air temperature $T_{\text {int }}(t)$ at different iterations on an initial guess of the constant interfacial temperature of $90^{\circ} \mathrm{C}$. 95

Figure 22: Objective functional versus iteration number for air injection (204 Darcy and Pressure $0.514 \mathrm{MPa}$ ). 96

Figure 23: The interfacial solvent temperature $\mathrm{T}_{\text {int }}(\mathrm{t})$ at different iterations on initial guess of periodic temperature variation between $90^{\circ} \mathrm{C}$ and $75^{\circ} \mathrm{C}$ after $20 \mathrm{~min}$ 97

Figure 24: Objective functional versus iteration number for air injection (204 Darcy and Pressure $0.514 \mathrm{MPa})$ 98

Figure 25: Experimental and calculated mass of live oil produced with time for air injection (Pressure 0.514 MPa, 204 Darcy) ............................................................................. 101

Figure 26: The interfacial air temperature $T_{\text {int }}(t)$ at physical model permeability of 427 Darcy and air pressure of $0.514 \mathrm{MPa}$ 103

Figure 27: Objective functional versus iteration number for air injection (427 Darcy and Pressure $0.514 \mathrm{MPa})$....... 104

Figure 28: Objective functional versus iteration number for air injection (87 Darcy and Pressure $0.514 \mathrm{MPa})$. 105

Figure 29: Objective functional versus iteration number for air injection (40 Darcy and Pressure $0.514 \mathrm{MPa})$. 106

Figure 30: Experimental and calculated heavy oil recovery at three different medium permeability 107

Figure 31: Variation of viscosity with air temperature at $0.514 \mathrm{MPa}$ absolute and 204 Darcy permeability of the physical model 116

Figure 32: Oil recovery versus time for different permeabilities at $0.514 \mathrm{MPa}$ absolute and $25^{\circ} \mathrm{C}$. (Solvent: Nitrogen) 118

Figure 33: Variation of production rate with model permeability at pressure of $0.514 \mathrm{MPa}$ absolute and temperature of $25^{\circ} \mathrm{C}$ (Solvent: Nitrogen) 119

Figure 34: Oil recovery versus time at $25^{\circ} \mathrm{C}$ nitrogen temperature, 204 Darcy model permeability, and different air pressures (absolute). 120

Figure 35: Oil recovery versus time at $0.514 \mathrm{MPa}$ absolute nitrogen pressure, 204 Darcy model permeability, and different nitrogen temperatures.... 121 
Figure 36: Oil recovery versus time at $0.514 \mathrm{MPa}$ absolute Nitrogen pressure, 204 Darcy model permeability, and constant $\left(90^{\circ} \mathrm{C}\right)$ as well as periodically varying nitrogen temperature in the range, $75-90^{\circ} \mathrm{C}$

Figure 37: Oil recovery versus time at $0.514 \mathrm{MPa}$ absolute nitrogen pressure, constant $\left(90^{\circ} \mathrm{C}\right)$ as well as periodically varying air temperature in the range, $75-90^{\circ} \mathrm{C}$, and 427 Darcy model

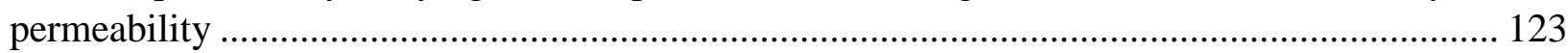

Figure 38: Oil recovery versus time at $0.514 \mathrm{MPa}$ absolute nitrogen pressure, constant $\left(90^{\circ} \mathrm{C}\right)$ as well as periodically varying air temperature in the range, $75-90^{\circ} \mathrm{C}$, and 87 Darcy model

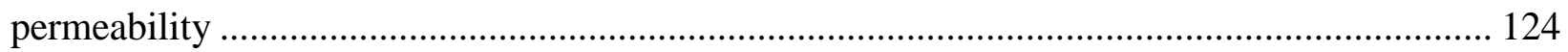

Figure 39: Oil recovery versus time at $0.514 \mathrm{MPa}$ absolute nitrogen pressure, constant $\left(90^{\circ} \mathrm{C}\right)$ as well as periodically varying air temperature in the range, $75-90^{\circ} \mathrm{C}$, and 40 Darcy model

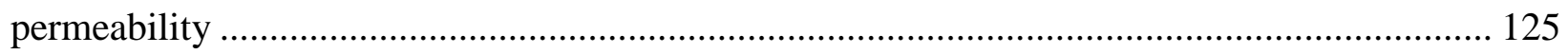

Figure 40: Dispersion coefficient function of nitrogen in heavy oil at different temperatures $\left(25^{\circ} \mathrm{C}\right.$, $50^{\circ} \mathrm{C}, 75^{\circ} \mathrm{C}$, and $90^{\circ} \mathrm{C}$ ) (Pressure $0.514 \mathrm{MPa}$; Permeability $204 \mathrm{Darcy}$ ) ................................ 126

Figure 41: Nitrogen objective functional versus iteration number (Pressure 0.514 MPa; Permeability 204 Darcy)

Figure 42: The nitrogen interfacial temperature $T_{\text {int }}(t)$ at different iterations of the optimal control algorithm under an initial guess of the constant temperature of $90^{\circ} \mathrm{C}$ 128

Figure 43: Nitrogen objective functional versus iteration number (204 Darcy and Pressure 0.514 $\mathrm{MPa})$.

Figure 44: Experimental and calculated mass of live oil produced with time (Pressure 0.514 MPa, 204 Darcy, Solvent Nitrogen)..... 131

Figure 45: Nitrogen objective functional versus iteration number (427 Darcy and Pressure 0.514 $\mathrm{MPa})$. 132

Figure 46: Nitrogen objective functional versus iteration number (87 Darcy and Pressure 0.514 $\mathrm{MPa})$.

Figure 47: Nitrogen objective functional versus iteration number (40 Darcy and Pressure 0.514 $\mathrm{MPa})$.

Figure 48: Experimental and calculated heavy oil recovery using Nitrogen at three different medium permeability 135 


\section{List of Appendices}

Appendix A: Porous Medium Permeability Calculation .................................................. 114

Appendix B: Solubility and Live Oil Density .............................................................. 115

Appendix C: Preliminary Experimental Results for Nitrogen Injection ............................... 117

Appendix D: Dispersion Coefficient Calculation for Nitrogen Injection .............................. 126

Appendix E: Optimal Control Policy for Nitrogen Interfacial Temperature.......................... 128

Appendix F: Permeability Effect on Optimal Policy for Nitrogen Injection ......................... 132 


\section{List of Symbols and Nomenclature}

\begin{tabular}{|c|c|}
\hline$A$ & area, $\mathrm{m}^{2}$ \\
\hline$C_{\mathrm{p}}$ & specific heat capacity, joules $/ \mathrm{g}{ }^{\circ} \mathrm{C}$ \\
\hline$D(\omega, T)$ & dispersion coefficient of solvent in media, $\mathrm{m}^{2} / \mathrm{s}$ \\
\hline$D_{\mathrm{p}}$ & diameter of the particle, $\mathrm{m}$ \\
\hline$g$ & gravity, $\mathrm{m} / \mathrm{s}^{2}$ \\
\hline$I$ & objective functional \\
\hline$J$ & augmented objective functional \\
\hline$K$ & permeability of the medium, Darcy \\
\hline$K_{\mathrm{r}}$ & relative permeability of the medium \\
\hline$k$ & thermal conductivity coefficient \\
\hline$m$ & cumulative mass of produced live oil, $\mathrm{g}$ \\
\hline$P$ & pressure, $\mathrm{MPa}$ \\
\hline$Q$ & volumetric fluid flow rate through the media, $\mathrm{m}^{3} / \mathrm{s}$ \\
\hline$r$ & distance along the radial direction, $\mathrm{m}$ \\
\hline$R$ & radius of the cylindrical medium, $\mathrm{m}$ \\
\hline$S$ & $\begin{array}{l}\text { finite differential surface area along r-direction, } \\
\mathrm{m}^{2}\end{array}$ \\
\hline$T$ & temperature, ${ }^{\circ} \mathrm{C}$ \\
\hline$T_{\text {int }}$ & $\begin{array}{l}\text { interfacial temperature of the solvent at the } \\
\text { solvent-heavy oil interface, }{ }^{\circ} \mathrm{C}\end{array}$ \\
\hline$t$ & time, $\min$ \\
\hline$t_{\mathrm{f}}$ & duration of the oil recovery experiment, $\min$ \\
\hline$V$ & volume of a finite element in the medium, $\mathrm{m}^{3}$ \\
\hline$v$ & Darcy velocity, $\mathrm{m} / \mathrm{s}$ \\
\hline$z$ & distance along the vertical direction, $\mathrm{m}$ \\
\hline$Z$ & $\begin{array}{l}\text { height of heavy oil inside the medium at a given } r \\
\text { and } t, m\end{array}$ \\
\hline$Z_{\mathrm{o}}$ & initial height, $\mathrm{m}$ \\
\hline
\end{tabular}


Greek Symbols

$\begin{array}{cl}\phi & \text { porosity of the medium } \\ \lambda_{1}, \lambda_{2}, \lambda_{3} & \text { costate variables } \\ \mu & \text { viscosity of the live oil, } \mathrm{mPa} . \mathrm{s} \\ \mu_{o} & \text { live oil viscosity coefficient, } \mathrm{kg} / \mathrm{m} . \mathrm{s} \\ \rho & \text { live oil density, } \mathrm{kg} / \mathrm{m}^{3} \\ \omega & \text { mass fraction of gas solvent in heavy oil } \\ \omega_{\text {int }} & \text { mass fraction of the gas solvent at the solvent- } \\ & \text { heavy oil interface } \\ \pi & \text { pi value }\end{array}$

Abbreviations

API

ASP

BFGS

CSS

EOR

PDE

MEOR

NMR

OOIP

ODE

SAGD

Vapex
American Petroleum Institute

Alkali-Surfactant-Polymer

Broyden-Fletcher-Goldfarb-Shanno Method

Cyclic Steam Stimulation

Enhanced Oil Recovery

Partial Differential Equation

Microbial Enhanced Oil Recovery

Nuclear Magnetic Resonance Spectroscopy

Original-oil-in-place

Ordinary Differential Equation

Steam Assisted Gravity Drainage

Vapor extraction 


\section{Chapter 1: Introduction}

\subsection{Crude Oil and Its Characteristics}

In general, crude oil is a mixture of liquid hydrocarbons of varying degree of volatility. It also contains some nitrogen, sulfur, and oxygen. ${ }^{1}$ Although, the percentage of each component may vary widely in different locations. Regardless of variations, however, almost all crude oil range from 82 to 87 percent carbon by weight and 12 to 15 percent hydrogen by weight. ${ }^{2}$

Crude oils are typically classified according to their viscosities and API (American Petroleum Institute) gravities, which is defined based on the ratio of the weight of equal volumes of a crude oil and pure water at standard conditions. Table 1 shows the grades of the crude oils based on API gravity. Furthermore, the types of crude oil reservoirs are conventional and unconventional based on their viscosities and API gravities. The viscosity of conventional oil reservoirs is lower than 100 centipoise $(\mathrm{cP})$ with API gravity of $20^{\circ}$ or greater, while unconventional oil reservoirs have the viscosity higher than $100 \mathrm{cP}$ with API gravity of $20^{\circ}$ or less. $^{2}$

Conventional oil reservoirs typically contain the highest quality, lightest oil, which can flow from underground reservoirs with comparative ease. On the other hand, unconventional oil reservoirs that have a high viscosity $\left(10^{4}-10^{6} \mathrm{cP}\right.$ or even higher $)$ are often tar-like, and require external forces for oil recovery. Some examples of unconventional oil reservoirs are oil shale, bitumen, heavy and extra-heavy crude oils, and deep-sea oils. ${ }^{3}$ 
Table 1: Classification of crude oil according to the API degree

\begin{tabular}{cc} 
Crude oil classifications & API degree $\left(^{(}\right)$ \\
\hline medium crude oil & higher than 31.1 \\
\hline medium crude oil & 31.1 to 22.3 \\
\hline heavy crude oil & 22.3 to 10 \\
\hline extra-heavy crude oil & Below 10
\end{tabular}

Typically, most heavy oil and natural bitumen are thought to be expelled from source rocks as light or medium oil and later converted to heavier components by bacterial degradation in subsurface reservoirs. ${ }^{4,5,6}$. Heavy oils are asphaltic, dense and viscous oils having an API gravity of between $10^{\circ}$ and $20^{\circ}$ API and a viscosity greater than $100 \mathrm{cP}$. Bitumen or tar sands share similar attributes to heavy oil but are yet more dense and viscous with API gravity less than $10^{\circ}$ API and viscosities usually greater than $10,000 \mathrm{cP}^{7}$

\subsection{Crude Oil and Energy Source}

With 90 million barrels of oil consumed worldwide daily, conventional oil reserves are being drawn down rapidly. New sources of hydrocarbon are required to supplement the decline of traditionally produced light crudes with low viscosity. Alternative sources of hydrocarbon energy include coal, shale production of oil and gas, heavy oil/bitumen and oil shale. ${ }^{8}$ The high viscosity of these non-conventional crude oils demand more energy intensive operations not only for their production and upgrading but also for transportation and consequently they are costlier to extract. However, yet, with estimated reservoirs of $3.3960 \times 10^{3}$ billion barrels of heavy oil (i.e., Venezuela in the Orinoco heavy-oil belt) ${ }^{9}$ and $5.5050 \times 10^{3}$ billion barrels of bitumen (i.e., Alberta, Canada), 
they represent alternative ample hydrocarbon resources for the oil production at an economicallyviable scale. ${ }^{10}$

The heavy oil and bitumen reservoirs in Canada, in particular, are one of the largest hydrocarbon resources in the world. ${ }^{2}$ Alberta's tar sand resides in three deposits (Athabasca, Cold Lake, and Peace River) and covers an area equal to the size of the province of New Brunswick. ${ }^{8,11}$ There are estimated 1.7-2.5 trillion barrels of bitumen in place in Alberta's oil sands. Canada's recoverable oil resource is second only to Saudi Arabia's.

At current production rates, resources from Alberta's tar sands could supply Canada's energy needs for more than 500 years, or the total world needs for up to 15 years and about $39 \%$ of Canada's total oil production is from oil sands. ${ }^{2}$

Currently, approximately 1.3 million barrels are produced per day and the production is expected to grow to three million bpd by $2020 .^{8,11}$ This fact indicates that heavy oil and bitumen reservoirs have tremendous potential for meeting the ever-increasing demands of energy and useful petroleum products. However, as it was mentioned previously, the main difficulty in the heavy oil and bitumen recovery is their immobility under reservoir conditions due to their high viscosity $\left(10^{4}-10^{6} \mathrm{cP}\right.$ or even higher). ${ }^{12}$

Thus, the active production of heavy oil and bitumen demands specific technological solutions that are economical as well as environmentally benign..$^{8,11,12}$ 


\subsection{Crude Oil Recovery}

In general, the production of crude oil from an underground reservoir can be classified in three terms: primary, secondary, and tertiary production.

The initial production of crude oil from an underground reservoir is accomplished by the use of natural reservoir energy. This type of production is termed primary products. Sources of natural reservoir energy that lead to major products include the swelling of reservoir fluids, the release of solution gas as the reservoir pressure declines, nearby communicating aquifers, and gravity drainage. ${ }^{11,13}$ However, when the natural reservoir energy declines, it becomes necessary to augment the natural energy with an external source, such as injection of fluids, either natural gas or water. The use of this injection scheme is called a secondary recovery operation. ${ }^{13}$ When water injection is used as the secondary recovery process, the process is referred to as water flooding.

The main purpose of either natural gas injection or a water injection process is to re-pressurize the reservoir and then to maintain the reservoir at a high pressure. ${ }^{11,13}$ Similarly, natural gases can be injected into the oil reservoir to maintain reservoir pressure. ${ }^{13}$ The recovery efficiency of a water flood is largely a function of the sweep efficiency of the flood and the ratio of the oil and water viscosities.

Sweep efficiency is a measure of how well the water has come in contact with the available pore space in the oil bearing zone. When an injected water is much less viscous than the oil it is meant to displace, the water could begin to finger, or channel, through the reservoir. This is referred to as viscous fingering and leads to significant bypassing of residual oil and lower flooding 
efficiencies. This bypassing of residual oil is an important issue in applying enhanced oil recovery techniques as well as in water flooding. ${ }^{8,11,13}$

Tertiary recovery processes were developed for application in situations in which secondary processes had become ineffective. However, the same tertiary processes were also considered for reservoir applications for which secondary recovery techniques were not used because of low recovery potential.

The tertiary recovery processes are also known as Enhanced Oil Recovery (EOR) processes. EOR processes attempt to create favorable conditions for improving oil recovery through (i) swelling of oil, (ii) increase in capillary number, (iii) reduction of the oil viscosity, (iv) reduction of the interfacial tension between the displacing fluid and the oil, (v) reduction of capillary forces in the reservoir, and (vi) alternation of the reservoir rock wettability. ${ }^{3,14}$

\subsection{Heavy Oil and Bitumen and Enhanced Oil Recovery}

Enhanced oil recovery (EOR) processes are used to recover heavy oil or reduce residual oil from reservoirs when oil cannot be either produced at all or improved economically using primary and secondary recovery processes. ${ }^{1,15}$

In general, EOR processes are classified into four main categories, thermal EOR, chemical EOR, gas EOR, and microbial EOR. ${ }^{3}$ Figure 1 represents four types of EOR processes, which are typically defined by the nature of the fluid injected into the reservoir. 


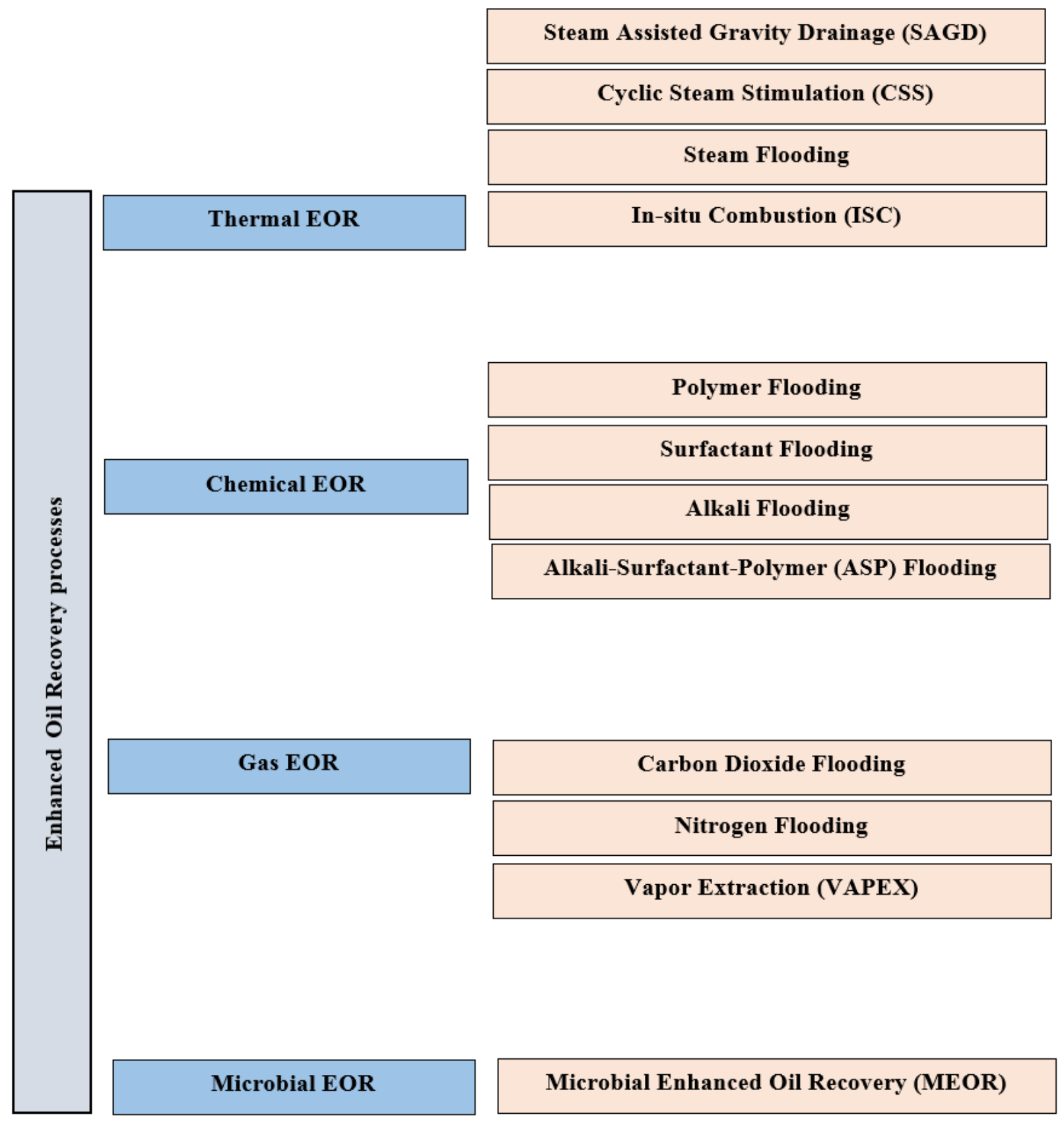

Figure 1: Classification of EOR processes

The total world oil production from EOR is about $3.5 \%$ of the total daily oil production of 85 million barrels. ${ }^{14}$ The majority of EOR oil production is from thermal methods contributing about 2 million barrels of oil per day. ${ }^{14}$ Thermal EOR relates to processes that require thermal energy 
through hot water, steam, or air injection to decrease the viscosity of heavy oil and bitumen. ${ }^{16}$ Thermal EOR process, particularly Steam Assisted Gravity Drainage or SAGD is the most common in-situ process presently used for heavy oil and bitumen recovery due to its simplicity and high recovery efficiency. It is noteworthy that SAGD accounts for $25-75 \%$ of the OOIP. ${ }^{8,17}$ For example, Alberta in 2009 produced 664,000 barrels per day of heavy oil using SAGD. ${ }^{11}$ However, thermal processes also face some inherent limitations. ${ }^{8,11,18}$ In thermal EOR, the steam is generated by burning natural gases; thus the cost of producing steam is very sensitive to increases in the price of natural gas. Furthermore, steam generation facilities are required and accounted for about $30 \%$ of the capital cost in SAGD. Also, steam production requires a significant source of water, usually from lake or aquifer. Additionally, most thermal EOR processes have high heat loss energy intensity and have large negative impacts on the environment. ${ }^{8,11}$ Despite well-insulated, the heat loss is inevitable, through the well casing, to the overburden and ground water below the reservoir. Moreover, greenhouse gas emissions and effluent water disposal pose significant challenges for these processes. ${ }^{2,8,11}$ It is important to note that over the last decade researchers have combined different EOR processes to enhance the production yield or reduce energy consumption or negative environmental impacts. One of the examples is Steam-Solvent processes.

Thermal EOR is followed by gas-based EOR, which contributes about a two-thirds of a million barrels of oil per day. ${ }^{14}$ Gas-based enhanced oil recovery (EOR) processes rely on the injection of gases such as carbon dioxide, nitrogen, and natural gas into heavy oil reservoirs to reduce inherent oil viscosity. The presence of a dissolved gas in the oil enhances the recovery by (i) creating a saturated displacement front by swelling oil in reservoir pores, and (ii) reducing the interfacial tension at the interface between the oil and water. ${ }^{12,19}$ As of today, carbon dioxide injection has 
gained considerable attention for oil recovery, especially in the US. ${ }^{20,21}$ In 2006 , about $37 \%$ of oil production in the US was contributed by carbon dioxide-based EOR, due to the availability of cheap carbon dioxide from natural sources. ${ }^{22}$ Some of the advantages of carbon dioxide are its high solubility in oil and availability from large and inexpensive natural gas sources. ${ }^{3,22-25}$ It has been reported that carbon dioxide EOR is economically attractive at oil prices around \$20 US per barrel. ${ }^{26,27}$ However, carbon dioxide injection faces many challenges including: (i) solvent loss caused by pipelines corrosion (ii) long start-up times and low initial rates oil recovery, (iii) high cost of capture and storage of carbon dioxide from air or other sources excluding natural sources, power plants or industrial sources at the current time..$^{3,22,23,28}$

The oil production from chemical EOR is estimated about a third of a million barrels of oil per day. Chemical EOR is a flooding process where chemical agents like polymer, surfactant, alkali or any combination [alkali-surfactant-polymer (ASP)] are used to either reduce the interfacial tension between the oil and the injected fluid or to improve the volumetric sweep efficiency. ${ }^{29,30}$ Polymers such as polyacrylamide and polysaccharides are used to improve sweep efficiency. ${ }^{29,31}$ The application of chemical flooding declined sharply in the US after 1988 due to rising prices of chemical flooding agents primarily surfactant. ${ }^{29,31,33}$ ASP flooding has received considerable attention in China in the last decade. ${ }^{29}$ Reservoir characteristics is another factor that limits the applicability of chemical EOR. For example, the reservoir features such as temperature, depth, salinity, and pH highly impact on the performance of chemical EOR. ${ }^{2}$

The heavy oil recovery using microbial injection process though is quite small due to lack of complete understanding of the mechanisms involved in microbial injection process and the impact 
of each device on different reservoir conditions ${ }^{35}$ It may be noted that EOR projects are strongly influenced by economics and crude oil prices. Therefore, since oil prices are expected to rise substantially in the future, EOR processes would become more economical to use. ${ }^{22,26}$

\subsection{Challenges and Research Objectives}

Among the non-thermal EOR processes, the important ones are the gas EOR processes. Over the years, many researchers have conducted various lab-scale experiments by using pure or mixture hydrocarbon gases (i.e., propane, butane, and hexane) injection for heavy oil recovery.

However, some of the major limitations of gas EOR are high costs and limited supplies of gas solvents, and solvent losses from oil reservoirs. Therefore, we have to look for those solvents for heavy oil recovery that are readily available at low costs, and are environmentally friendly.

Based on aforementioned considerations, atmospheric air injection is an potentially viable process

for heavy oil recovery because of its free availability. ${ }^{34,35}$ As a result, the first objective of this study is to investigate the applicability of freely atmospheric air as a solvent for heavy oil recovery under moderate and non-reactive temperature and pressure conditions in a solvent-assisted gravity drainage process.

For this purpose, experiments are performed with a lab-scale reservoir model and air injection at sufficiently low temperatures $\left(25^{\circ} \mathrm{C}-90^{\circ} \mathrm{C}\right)$ and pressures $(0.169-0.514 \mathrm{MPa})$ (absolute) to preclude any hydrocarbon oxidation. 
The second objective of this study is to prove experimentally that oil production can be increased by suitable varying air injection temperature with time. The air interfacial temperature versus time is considered as a control function.

The third objective of this study is to develop a robust mathematical model of the experimental recovery process, utilize it to determine the optimal control function, and validate the function.

\subsection{Structure of the Dissertation}

This dissertation is organized into six chapters as follows.

Chapter 1: This chapter provides a brief background of crude oil and different processes of heavy oil recovery. Also, the research objectives and organization of the text are introduced.

Chapter 2: In this chapter, literature review is provided on gas EOR and the impact of different factors (i.e., viscosity) on gas EOR performance. This chapter also presents optimal control applications for enhanced oil recovery.

Chapter 3: The experimental methods and procedures are explained in this chapter.

Chapter 4: This chapter presents optimal control of the air-based gas EOR. The optimal control is formulated. The necessary conditions are derived for the maximum of the heavy oil recovery. An optimal control algorithm is developed to determine optimal air interfacial temperature versus time policy to maximize oil production.

Chapter 5: In this chapter, the experimental and numerical simulation results are presented, analyzed and discussed in details.

Chapter 6: This chapter summarizes the contributions of this research, and identifies recommendations for future work. 


\section{Chapter 2: Literature Review}

As previously mentioned, gas EOR processes rely on the injection of gases such as carbon dioxide into heavy oil reservoirs to reduce native oil viscosity. The following sections discuss in detail about the mechanism of gas EOR, the factors that impact heavy oil recovery, and optimal control application for enhanced oil recovery.

\subsection{Mechanism of Gas EOR}

The mechanism of gas EOR is based on the phenomenon of viscosity reduction of heavy oil in the presence of the absorbed solvent (in this case atmospheric air injected), which is predominantly nitrogen. ${ }^{36,37}$ The absorbed solvent facilitates oil recovery by reducing the interfacial tension between the oil and water, and creating a saturated displacement front by swelling oil in the reservoir pores. As a result, the heavy oil reservoirs become mobile and flow under gravity for easy recovery. ${ }^{12}$ Furthermore, the gas solvent mixes with the heavy oil phase through combined phenomena of molecular diffusion, viscosity reduction, capillary action, gravitational flow, and surface renewal. The net effect is dispersion, which cannot be described by solvent diffusivity alone. $^{38}$

\subsection{Factors that Influence Gas EOR}

Some of the relevant parameters and factors that impact gas EOR, such as viscosity, diffusion, dispersion, and permeability of the heavy oil reservoir are discussed next. 


\subsubsection{Heavy Oil Viscosity and Gas EOR}

As indicated before, one of the biggest challenges of heavy oil recovery is the high viscosity of heavy oil that does not flow spontaneously and cannot be produced efficiently with conventional primary and secondary oil recovery processes. ${ }^{1,15}$ In gas EOR, the gaseous solvent injection results in viscosity reduction. The viscosity of heavy oil and bitumen is a strong function of temperature in comparison with pressure, but while gases are dissolved in heavy oil and bitumen, pressure can influence the viscosity. ${ }^{1,15}$ In general, heavy oil viscosity is the function of temperature, the concentration of dissolved gases, pressure, and asphaltene. Lederer has reported that the correlation for dependence of viscosity on the solvent concentration as ${ }^{39}$

$\mu_{\text {mix }}=\mu_{s}^{f s} \mu_{B}^{f B}$

where

$f_{B}=\frac{\gamma C_{B}}{\gamma C_{B}+C_{S}} ; f_{s}=1-f_{B}$

where $\gamma$ is a weight factor having a value between zero and unity, $f_{B}$ is a weighted fraction of the more viscous component, $\mu_{B}$ and $\mu_{s}$ are the viscosities (Pa.s) of bitumen and the injected solvent respectively, and $C_{B}$ and $C_{S}$ are the volume fraction of bitumen and solvent, respectively. ${ }^{39}$ Das and Butler (1996) used the two parameters viscosity and temperature, and their correlation as given below: ${ }^{40}$

$\log _{10} \log _{10}(\mu+0.7)=b_{1}+b_{2} \log _{10} T$

where $\mu$ is the viscosity of Peace River bitumen (Pa.s), T is the absolute temperature $\left({ }^{\circ} \mathrm{K}\right)$, and $\mathrm{b}_{1}$ and $b_{2}$ values are 9.5235 and -3.5723 , respectively. ${ }^{40}$ Jin (1999) also developed the empirical 
correlation between the viscosity of produced oil with butane solvent concentration and the correlation was: ${ }^{41}$

$\mu\left(C_{s}\right)=16609 C_{s}^{-2.12}$

$\mu\left(\omega_{s}\right)=0.0094655 \omega_{s}^{-2.12}$

where $\mu$ is the heavy oil or bitumen viscosity in poise, $C_{s}$ is the volume fraction of butane in the heavy oil, and $\omega_{s}$ is the mass fraction of butane in the heavy oil. ${ }^{41}$

\subsubsection{Diffusion and Gas EOR}

Solvent gas diffusion in the heavy oil and bitumen is the primary reason for viscosity reduction, and hence it affects the production rate, ${ }^{40}$ which makes diffusion phenomenon most important in gas EOR process. Diffusivity of injected gas solvent (i.e., air) can be determined by either experimental methods or by empirical correlations. ${ }^{42}$ The experimental methods are classified under direct and indirect method. In the direct method, there would be a compositional analysis of heavy oil sample extracted at different times. Indirect methods are classified into two categories:

1. Based on property change such as volume, pressure, solute volatilization rate, the position of the gas-light interface, etc.

2. Diffusivity measurement from self-diffusion coefficients which are measured by Nuclear Magnetic Resonance Spectroscopy (NMR). ${ }^{38}$

Hayduk and Cheng (1971) gave a relationship between diffusivity and solvent viscosity: ${ }^{43}$

$D=\alpha \mu^{-\beta}$ 
where $\alpha$ and $\beta$ are constants for each diffusive substance. The diffusivity of propane and butane in the Peace River bitumen were estimated using an indirect method by Das and Butler (1996). ${ }^{40}$ They obtained the optimized constant values $\alpha$ and $\beta$ in the Hayduk and Cheng (1971) correlation, for propane and butane to measure the diffusion coefficients. The empirical relationship for butane determined as:

$D_{s}=4.1300 \times 10^{-10} \mu^{-0.46}$

Regarding to propane, the correlation was found as:

$D_{s}=1.3060 \times 10^{-9} \mu^{-0.46}$

Upreti and Mehrotra $(2000,2002)$ estimated the diffusivity of carbon dioxide $\left(\mathrm{CO}_{2}\right)$, methane $\left(\mathrm{CH}_{4}\right)$, ethane $\left(\mathrm{C}_{2} \mathrm{H}_{6}\right)$, and nitrogen $\left(\mathrm{N}_{2}\right)$ in Athabasca bitumen, in the range of $25^{\circ} \mathrm{C}$ to $90^{\circ} \mathrm{C}$ at 4 and $8 \mathrm{MPa}$. They used an indirect non-intrusive pressure decay experimental method to find the diffusivity of these gases as a function of gas concentration in bitumen. They developed the following correlation for average gas diffusivity: ${ }^{12,19,42}$

$\ln D=d_{o}+d_{1}(T+273.15)$

They observed that diffusivity is a function of gas concentration in bitumen, and at a given gas concentration and pressure, the diffusivity increases with temperature. Lastly, their results indicate that gas diffusivity increases with pressure at a given temperature and gas concentration. 


\subsubsection{Dispersion and Gas EOR}

Diffusion is a special case of dispersion where the fluid is stationary. Hence, the dispersion is a combined effect of diffusion and convective motion. At a macroscopic scale, this convective transport is Darcy's law describes a porous media. The variation in the reservoir properties results in a macroscopic dispersion. When the fluids are moving through porous media, the dispersion coefficient increases due to convective mixing and the dispersion may be higher than that due to diffusion alone. ${ }^{44}$ Dispersion in porous media comprises concentration gradient in both longitudinal and transverse to the direction of solute-solvent flow. ${ }^{19,42}$

\subsubsection{Permeability of the Reservoir and Gas EOR}

In general, the reservoir volumes consist of sandstone which is considered as high porous and high permeable media. Porosity is a measurement of the fraction of bulk volume occupied by accessible pore space. Equation (10) shows the relationship between porosity and the volume fraction.

Permeability describes the fluid conductivity through porous media. Permeability is directly related to the porosity, and it depends on the porous connectivity and the size of the porous volumes. Permeability can be calculated by Darcy's law which was developed semi-empirically by Darcy in the $19^{\text {th }}$ century for single phase flows and in the 20th century for multiphase flows. ${ }^{45}$ The permeability coefficient depends on both material and the fluid properties. The greater the $K$ value is, the higher will the flow rate be. ${ }^{46}$ Darcy's law is expressed by:

Porosity $(\varnothing)=\frac{\text { Volume of the void space }\left(V_{V}\right)}{\text { Total or bulk volume of the rock (or glass bead) }\left(V_{T}\right)}$ 
$Q=\frac{K \Delta P}{\eta \Delta L} A$

where $Q$ is the flow rate, $K$ is the permeability coefficient, $\Delta P$ is the pressure difference, $A$ is the cross-sectional area of the flow, $\eta$ is the fluid viscosity, and $\Delta L$ is the flow length. The permeability is expressed in Darcy or millidarcy, and most of the oil reservoirs are in the range of ten to several hundreds of millidarcy.

\subsection{Optimal Control and Enhanced Oil Recovery}

An optimal control is a function that optimizes the performance of a system changing with time, space, or any other independent variable. ${ }^{48}$ As a subject, optimal control is a superset of optimization that deals with the determination control functions for a given system that will optimize a particular performance criterion subject to constraints describing the system dynamics.

Optimal control has applications in many different fields, including aerospace, process control, and engineering. Only simple optimal control problems were solved before the 1950s. The revolution of the digital computers in the 1950s allowed the application of optimal control theory and methods to complex problems. Many applications of optimal control theory to enhanced oil recovery methods were developed. ${ }^{48}$

For example, optimal control was used to determine the concentration-dependent dispersion of propane in vapour extraction of heavy oil. ${ }^{48}$ In more detail, the necessary conditions for the minimum were fundamentally derived, utilizing optimal control. A computational algorithm is formulated to calculate the propane dispersion function simultaneously with the propane-heavy oil 
interfacial mass fraction. The results show that dispersion of propane is a unimodal function of its concentration in heavy oil, and lies in the range, $0.50000 \times 10^{-5}-7.9930 \times 10^{-5} \mathrm{~m}^{2} / \mathrm{s}$.

In a similar case, optimal control was utilized to enhance the oil production of vapour extraction using propane and butane injection pressure versus time as the control function. The results showed the optimal policy successfully lead to a $20-35 \%$ increase in the experimental oil production with propane and butane as pure solvents, and heavy oil of $14,500 \mathrm{mPa} \cdot \mathrm{s}$ viscosity in lab scale reservoirs of 25 and $45 \mathrm{~cm}$ heights, and 204 Darcy permeability. Lastly, according to the results, the experimental oil production values from the optimal policy were within $\pm 5 \%$ of those predicted by the optimal control algorithm. ${ }^{49}$

Until now, many researchers have done different experiments, and mathematical modeling to have a better understanding of transport mechanism in gas EOR as well as recognize critical parameters to increase the heavy oil recovery performance (control functions such as dispersion coefficient and solvent injection pressure versus time). ${ }^{48,49,60}$

However, to the best of our knowledge, no one has applied optimal control policy by utilizing solvent interfacial temperature versus time as a control function to maximize oil production in gas EOR. 


\section{Chapter 3: Experimental Setup}

The sections below describe experimental setups and procedures followed in this study to investigate the impacts of atmospheric air injection on heavy oil recovery. The first experimental setup is used for determination of the effects of permeability, air pressure, and air temperature on heavy oil recovery. The second setup is used for determining the concentration of air at the heavy oil interphase during air injection process. This setup is used to gather the data at different temperatures.

\subsection{Air Injection Experimental Setup}

Figure 2 shows the schematic of the experimental setup used to recover heavy oil from a cylindrical reservoir model by injecting air at different temperatures and pressures. This setup allows to generate heavy oil production data with constant and varying air temperature with time. The setup consists of a cylindrical pressure vessel with an internal diameter of $15 \mathrm{~cm}$ and height of $80 \mathrm{~cm}$. A resistance temperature detector (Rugged Transition Joint Probe, Newport Electronics, CA, US) and a pressure transducer (Voltage Output Pressure Transducer, PX01C1-200G5T, Omega Inc., Canada) are provided to measure, respectively, the air temperature and pressure inside the vessel. Also, a digital thermometer (VWR traceable digital thermometer with recorder output, Mississauga, Ontario, Canada) is installed to record the interfacial temperature of the physical model.

Inside the vessel and suspended from a load cell (Miniature Load Cell Model 31, Honeywell Sensotec, OH, US) is a physical reservoir model (of $3 \mathrm{~cm}$ radius and $25 \mathrm{~cm}$ length), which is 
brought into contact with air during an experiment. The load cell tracks the decrease in the mass of the physical model with time as the oil is produced. The live oil production was recorded online every 5 seconds with data acquisition system from the load cell, which exhibited reduction in the weight of the model. The physical models are prepared from Fort Kent heavy oil (supplied by Saskatchewan Research Council, Alberta, Canada) of viscosity $14,500 \mathrm{mPa} . \mathrm{s}$ at $20^{\circ} \mathrm{C}$ and glass beads (BALLOTINI® Impact Beads, Potters Industries LLC, PA, US) packed together in a cylindrical stainless steel wire mesh. The models are homogeneous with a porosity around 0.38 and have different permeability values, namely, 40, 87, 204, and 427 Darcy.

A flow meter (In-line flow meters, FL46302, Omega Inc., QC, Canada) monitors the flow of air to the pressure vessel. To maintain a desired air pressure inside the pressure vessel at any time two pressure control valves (PV101-10V, Omega Inc., QC, Canada) are used, respectively, at the air entrance and the air exit. The air inside the pressure vessel is heated using a heating tape (Heat tape with the controller, HTWC 101, Omega Inc., QC, Canada) wrapped around the pressure vessel. The pressure vessel is covered with an aluminum wrap to minimize the heat loss to the surrounding. All instruments are connected via a data acquisition system to a computer and interfaced with LabView version 7.1 software. To be able to cool down the air temperature inside the vessel, cold water is circulated through a copper coil (40 inches long and with 0.25 -inch tube diameter) wrapped around the vessel.

To collect the heavy oil recovered in an experiment, the pressure vessel has a small funnel connected directly to a calibrated collection tube. A load cell (Mid Range Precision Miniature Load Cell model 31, Honeywell Sensotec, Columbus, $\mathrm{OH}$ ) is attached to the calibrated collection tube. A digital thermometer (VWR $®$ Traceable $®$ Digital Thermometer with Recorder Output, ON, 
Canada) upstream of the collection tube helps to monitor and record the temperature in the pressure vessel. A collection tube sends the recovered oil to a viscometer (VISCO PRO 2000, Cambridge Viscosity, Inc., MA, US). The recovered oil is then directed to a stainless steel flash tank of $300 \mathrm{~cm}^{3}$ capacity.

The tank is heated to $70^{\circ} \mathrm{C}$ for 30 minutes with the help of the heating tape to separate the dissolved air from the oil. This air is then routed to two water columns in series, which are used to measure the amount of dissolved air in oil.

The columns are made of acrylic and have capacities $2.6000 \times 10^{3} \mathrm{~cm}^{3}$ and $2.9000 \times 10^{3} \mathrm{~cm}^{3}$, respectively. The first column is filled with water. The second column is calibrated and used to collect the water displaced from the first column when the air is separated. Table 2 shows the range of parameters used in this study. The temperature above $90^{\circ} \mathrm{C}$ was avoided to preclude any hydrocarbon oxidation (please see Section 5.1.5). The range was selected based on the recent research on gas EOR. ${ }^{48-50}$

Table 2: Parameters range used in this study

\begin{tabular}{cc} 
parameter & range \\
\hline temperature & $25-90^{\circ} \mathrm{C}$ \\
\hline pressure & $0.169-0.514 \mathrm{MPa}$ absolute \\
\hline permeability & $40-427$ Darcy
\end{tabular}




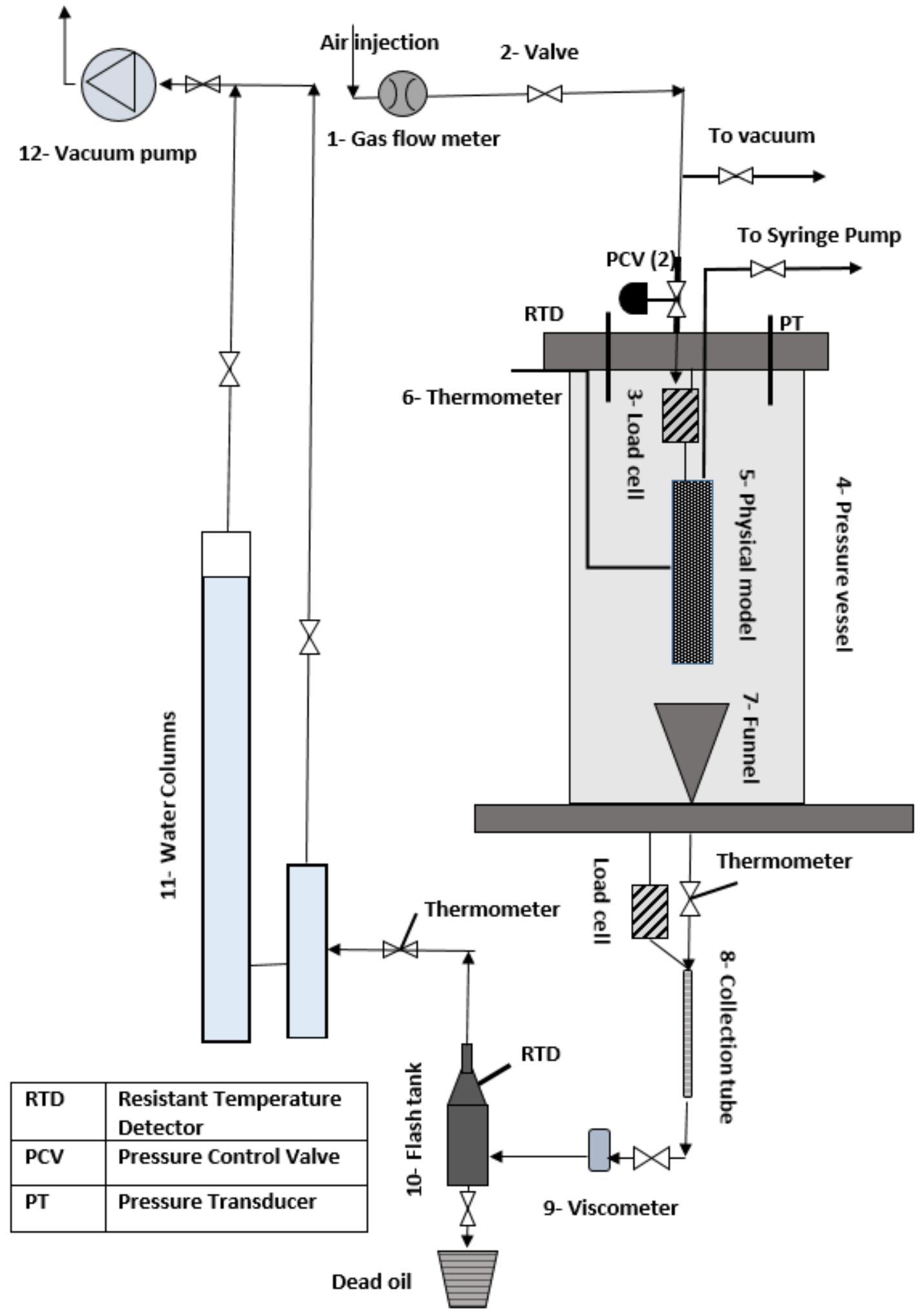

Figure 2: Schematic diagram of the experimental setup. The list of equipment in order are: 1-gas flow meter, 2-valve, 3-load cell, 4- pressure valve, 5- physical model, 6- thermometer, 7-funnel, 8-collection tube, 9- viscometer, 10- flash tank, and 11-water columns 


\subsection{Experimental Procedure}

Before starting each experiment, the air was purged from the pressure vessel by applying vacuum close to $0.1 \mathrm{MPa}$ (absolute) using a vacuum pump. Then the atmospheric air was injected into the pressure vessel at different pressures $(0.169,0.286,0.403$ and $0.514 \mathrm{MPa}$ absolute) and temperatures $\left(25-90^{\circ} \mathrm{C}\right)$.

The run time for each experiment was 430 minutes (a little over seven hours). At the start of each experiment, the initial air flow rate to the pressure vessel was set at $2.5 \mathrm{~L} / \mathrm{min}$ and then gradually decreased to $0.13 \mathrm{~L} / \mathrm{min}$.

The penetration of air into the physical model eventually led to the production of "live oil," which drained out from the bottom of the model by gravity and accumulated in the collection tube. After a certain amount of live oil had been collected in the collection tube, the live oil was directed through the viscometer to the flash separation tank preheated to $70^{\circ} \mathrm{C}$ for about 30 minutes to separate the air dissolved in the oil.

As the separated air moved from the flash tank to the first water column, the water level of the second column started to rise. The increase in the level was recorded. The valve on the top of the first column was then opened to release the air to the fume hood. The air-free "dead oil" from the flash tank was weighed and recorded. At the end of an experiment, the air flow was shut, and the pressure vessel was vented to atmosphere. A photo of the experimental setup is shown in Figure 3. 


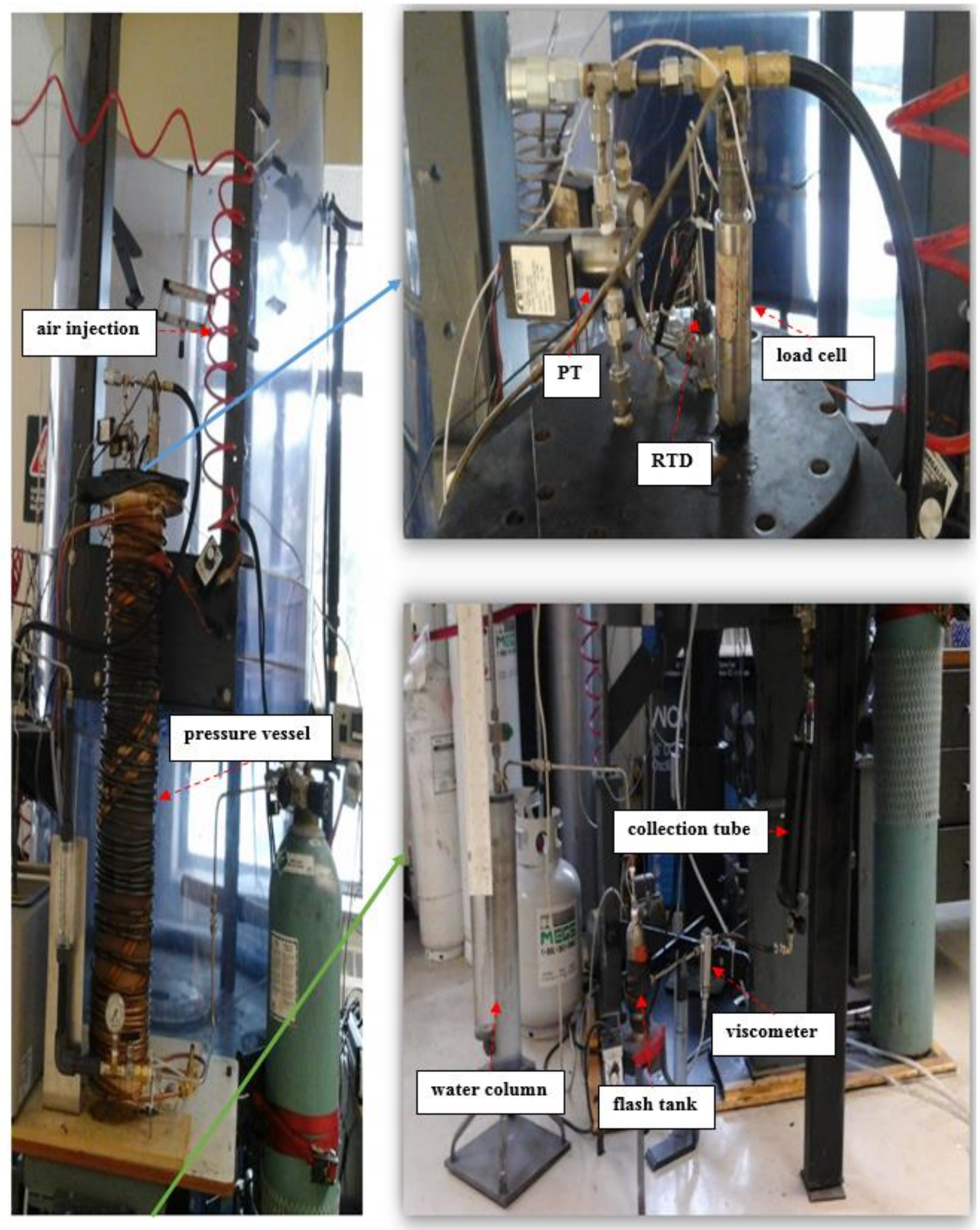

Figure 3: Picture of Air Injection Experimental Set 


\subsection{Physical Model Preparation}

The physical model was made of heavy oil of a viscosity of $14,500 \mathrm{mPa} . \mathrm{s}$ at $20^{\circ} \mathrm{C}$. Oil-saturated porous medium with glass beads of known permeability was packed in a cylindrical wire mesh of $3 \mathrm{~cm}$ radius and $25 \mathrm{~cm}$ length. The oil packing was prepared using a known mass of heavy oil, $602.86 \mathrm{~g}$, which was placed in a temperature-controlled heater. The heavy oil was heated for 30 minutes at $70^{\circ} \mathrm{C}$, for sufficient reduction in oil viscosity to promote glass beads mixing. Glass beads were gradually added to the heated heavy oil to ensure proper mixing without trapping air bubbles.

After heavy oil was thoroughly mixed with the glass beads, the saturated mixture was packed into the cylindrical wire mesh physical model. The saturated mixture was slowly poured inside the cylindrical wire mesh as a thin layer, and allowed to settle under gravity. Then another layer oil was added. This process was repeatedly to fill the wire mesh. This method of preparing oilsaturated beads ensured that the medium was homogenous, and did not trap any air. After the entire mesh was packed, it was weighed and left at room temperature for one day to reach thermal equilibrium (at about $23^{\circ} \mathrm{C}$ room temperature) prior to the experiments.

Porosity is a measurement of the fraction of bulk volume occupied by accessible pore space. The porosity of the packing of different glass beads was measured by the imbibition or saturation method. A cylindrical model was filled with the glass beads and weighed. The cylindrical model was flooded with water from the bottom until it is saturated. The cylindrical model filled with water was again weighed. The porosity of glass beads in the cylindrical model was determined from the amount of water in the cylindrical model. Table 3 lists the properties of the glass beads 
used for the physical model preparation. Knowing the density of the glass beads and density of the heavy oil, the porosity of the physical model was measured to be 0.38 .

Table 3: Properties of glass beads used in this study

\begin{tabular}{ccc} 
size range $(\mathrm{mm})$ & porosity & permeability (Darcy) \\
\hline $0.84000-0.59400$ & 0.38500 & 427 \\
\hline $0.59400-0.41900$ & 0.38000 & 204 \\
\hline $0.41900-0.24800$ & 0.37800 & 87 \\
\hline $0.24800-0.21000$ & 0.37600 & 40
\end{tabular}

\subsubsection{Permeability of Physical Model}

We prepared physical models of different permeabilities to investigate the impact of permeability on heavy oil recovery. The procedure of permeability measurement was adopted by El-Haj (2007). ${ }^{71}$ To measure the permeability of the porous medium made of heavy oil and glass beads, a vertical acrylic cylinder of $6 \mathrm{~cm}$ internal diameter and $21 \mathrm{~cm}$ height was used. The cylinder had two ports, one at the air entrance and the other for the air exit. A differential pressure transducer (Differential Pressure Transducer, PX409, Omega Inc., QC, Canada) was used to connect the inlet and outlet ports to measure the pressure difference across the media as air passed through the porous medium. The flow rate of air at the outlet was measured by a flow meter (In-line flow meters, FL46302, Omega Inc., QC, Canada). The permeability of the porous medium is given by ${ }^{49}$ $K=\frac{Q \mu \Delta x}{A \Delta P} \times 1.01325 \times 10^{12}$ 
where $Q$ is the volumetric fluid flow rate through the media, $A$ is the cross-section area of fluid flow through the medium, $\mu$ is the dynamic viscosity of the fluid, and $\Delta P$ is the pressure difference across the medium of thickness $\Delta x$. The values of these parameters are listed in Table 4 for glass beads of size in the range $0.248-0.210 \mathrm{~mm}$. With these values, Equation (12) yields the permeability of 40 Darcy.

Table 4: Parameters for calculation of permeability for glass bead size $0.248-0.210 \mathrm{~mm}$

\begin{tabular}{cc} 
parameter & value \\
\hline$Q\left(\mathrm{~m}^{3} / \mathrm{s}\right)$ & $1.66001 \times 10^{-5}$ \\
\hline$\mu$ at $20^{\circ} \mathrm{C}(\mathrm{Pa} . \mathrm{s})$ & $1.8401 \times 10^{-5}$ \\
\hline$\Delta x(\mathrm{~m})$ & 0.21000 \\
\hline$A\left(\mathrm{~m}^{2}\right)$ & $2.82000 \times 10^{-3}$ \\
\hline$\Delta P(\mathrm{~Pa})$ & 577.00
\end{tabular}

\subsection{Solvent Gas Solubility and Live Oil Density}

As previously mentioned, the collected live oil was delivered to stainless steel flash separation tank $\left(300 \mathrm{~cm}^{3}\right)$ to separate the solvent gas from oil by heating the flash tank to $70^{\circ} \mathrm{C}$. After a particular time, the amounts of absorbed gas transfer to gas measuring columns initially filled with water, and the residual oil (deal oil) is collected and measured.

The mass of the solvent gas was determined as follows. A known amount of live oil was transferred to the flash tank and heated at maintained temperature of $70^{\circ} \mathrm{C}$ to ensure proper flashing of the gas. 
The flashed gas was delivered to the first water column. Wherein, the water was displaced resulting in a rise of the water level in the second water column. After 10 minutes the differential pressure reading approached zero with no more displacement in the second water column. The displaced volume of water determined the gas volume. The valve on the top of the first water column was opened to vent the gas, and the net amount of dead oil was collected by opening the valve at the bottom of the flash tank. By knowing the amount of air dissolved in oil, the solvent gas-free oil (dead oil) weight, and the volume of the live oil, the solubility of the solvent gas was determined as well as the live oil density using the following formulas:

$\omega=\frac{m_{\text {gas }}}{m_{\text {gas }}+m_{\text {oil }}}$

$\rho=\frac{m_{\text {gas }}+m_{\text {oil }}}{V_{\text {Live oil }}}$

where $\omega$ is the dissolved mass fraction of the solvent gas, $m_{\text {gas }}$ is the mass of liberate solvent gas, $m_{\text {oil }}$ is the mass of the dead oil, $\rho$ is the live oil density, and $V_{\text {Live oil }}$ is the volume of live oil. A sample of air solubility and live oil density calculations is presented in Appendix B.

\subsection{Interfacial Solvent Concentration Setup}

This experimental setup was designed to determine the air interfacial concentration at different pressures and temperatures. This innovative part consists of a high-pressure vessel, syringe pump, water bath, data acquisition system, and capillary tubes for the sample oil collection. It is important to mention that the purpose of these data is to derive the empirical correlation between the interfacial mass fraction of air $\left(\omega_{\text {int }}\right)$ and the interfacial temperature of air $\left(T_{\text {int }}\right)$. This correlation furnishes the boundary condition of the mass transfer model. 


\subsubsection{Experimental Procedure and Interfacial Solvent Concentration}

Figure 4 shows the experimental setup for determination of atmospheric air concentration at the heavy oil interfacial. Before starting lab experiment, the air was purged from the pressure vessel by applying vacuum close to $0.1 \mathrm{MPa}$ (absolute) using a vacuum pump. Then, the pressure vessel was filled with $25 \mathrm{~g}$ of heavy oil of viscosity 14,500 mPa.s. The filling up the pressure vessel was done slowly to avoid entrapment of air within heavy oil layers. After thoroughly sealing the pressure vessel it was placed in a water bath. The objective of the water bath was to maintain the temperature at a set temperature. The top end of the vessel was connected to the syringe pump and the capillary tube that was used to take the oil sample from the top most layers, very close to the heavy oil surface (about one millimeter) into a sealed collection tube. The run time for each test was 430 minutes (a little over seven hours).

At the start of each experiment, the initial air flow rate to the pressure vessel was set at $0.13 \mathrm{~L} / \mathrm{min}$. We have also installed a digital thermometer (VWR Traceable Digital Thermometer with recorder output, Mississauga, Ontario, Canada) to record the interfacial temperature of the heavy oil. After collecting the live oil inside a sealed sample tube, the sample was weighted. Then the tube was open to atmosphere and heated to around $70^{\circ} \mathrm{C}$ to release the dissolved and residual air in the oil. After flushing the solvent gas out the tube the dead oil was weighed. From the mass balance, air mass fraction in the oil was calculated. The experiments were performed at different injection pressures and temperatures. The solvent concentration thus obtained is the interfacial concentration used in this study. 
Table 5 lists the interfacial air mass fraction at different temperatures at the constant pressure of $0.514 \mathrm{MPa}$.

Table 5: Temperature versus interfacial concentration for air at pressure of $0.514 \mathrm{MPa}$

\begin{tabular}{cc} 
temperature $\left({ }^{\circ} \mathbf{C}\right)$ & air mass fraction $\left(\omega_{\text {int }}\right)$ \\
\hline 25 & 0.015601 \\
\hline 50 & 0.061102 \\
\hline 75 & 0.16603 \\
\hline 90 & 0.46301
\end{tabular}

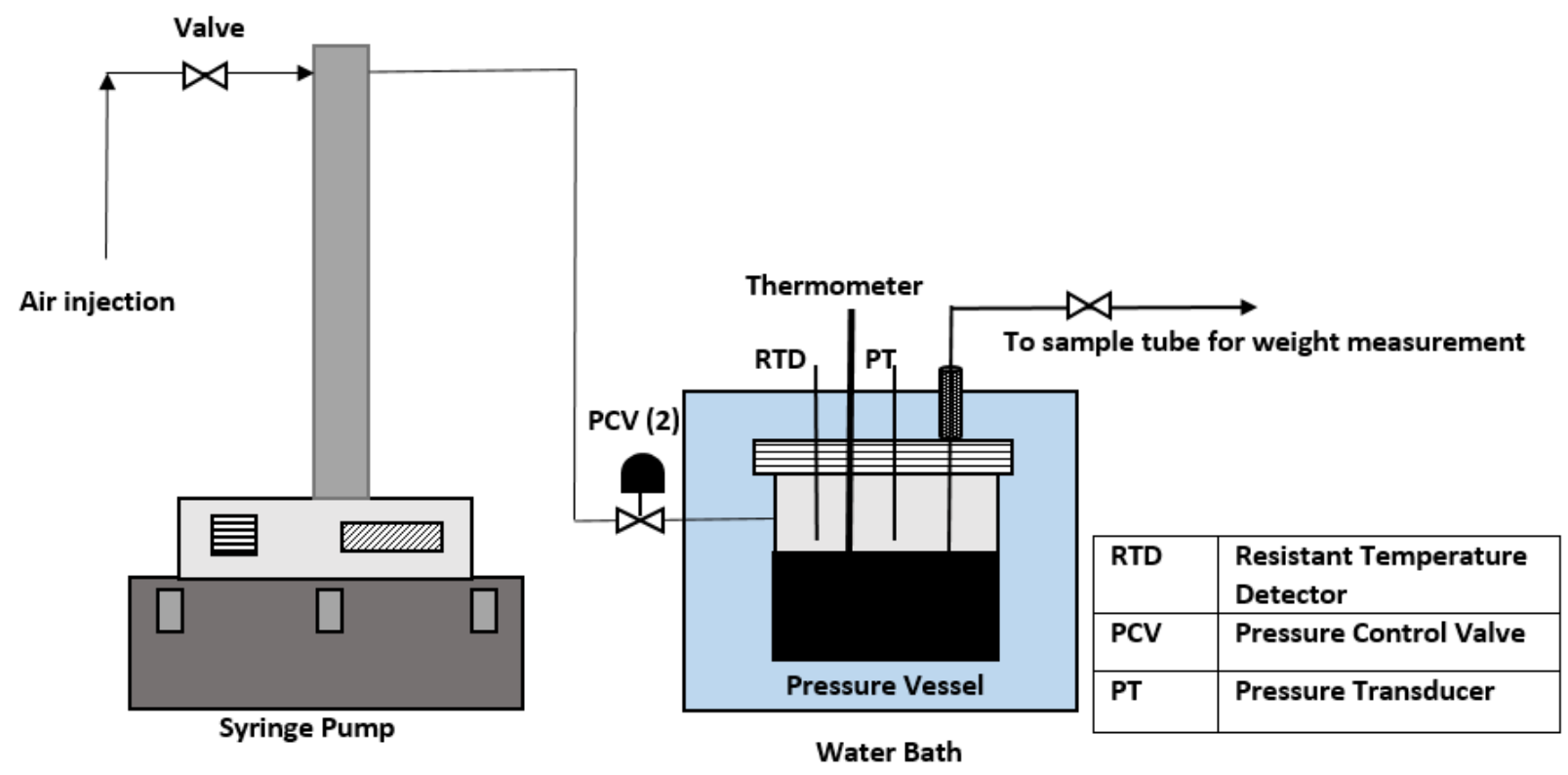

Figure 4:Schematic of solvent-heavy oil system for the measurement of interfacial solvent concentration 


\subsection{Experimental Results Repeatability}

The accuracy of this study was based on data collected from load cell weight change during the experiments. Therefore, it was essential to calibrate the load cell at the start of each experiment and compare its readings with scalar at the beginning and end of each experiment.

Also, the second load cell was used to have a better control of heavy oil recovery during each

experiment. Lastly, the relative errors between the oil recovery from the repeated experiments were found to be in the range of $1.3-1.5 \%$. 


\subsection{Laboratory Equipment}

A list of the instrumentation and laboratory equipment used in this study is shown in Table 6 .

Table 6: List of equipment used in this study

accuracy

instrument

information

range

(Error \%)

\begin{tabular}{|c|c|c|c|}
\hline $\begin{array}{c}\text { Resistance Temperature } \\
\text { Detector }\end{array}$ & $\begin{array}{c}\text { Rugged transition joint } \\
\text { probe, Newport Electronics, } \\
\text { Santa Ana, CA }\end{array}$ & $\begin{array}{c}0-230^{\circ} \mathrm{C} \\
0-2500 \text { (psig) }\end{array}$ & $\begin{array}{c}0.1 \% \text { (Full } \\
\text { scale) }\end{array}$ \\
\hline Pressure Transducer & $\begin{array}{c}\text { Voltage output pressure } \\
\text { transducer, PX01C1-200G5T, } \\
\text { Omega, Inc., } \\
\text { Laval, Quebec, Canada }\end{array}$ & $\begin{array}{l}-46-121^{\circ} \mathrm{C} \\
0-200(p s i g)\end{array}$ & $0.05 \%$ \\
\hline Load Cell & $\begin{array}{c}\text { Mid Range Precision } \\
\text { Miniature Load Cell } \\
\text { model } 31, \text { Honeywell } \\
\text { Sensotec, Columbus, OH, } \\
\text { USA }\end{array}$ & $\begin{array}{l}1000 \mathrm{~g} \text { to } 1000 \mathrm{lb} \\
-53-121^{\circ} \mathrm{C}\end{array}$ & $\begin{array}{l}0.15 \% \\
\text { (of full load) }\end{array}$ \\
\hline Flow Meter & $\begin{array}{c}\text { In-line flow meters, FL46302, } \\
\text { Omega, Inc., Laval, } \\
\text { Quebec, Canada }\end{array}$ & $\begin{array}{c}-10-65^{\circ} \mathrm{C} \\
150 \mathrm{psig}(\max )\end{array}$ & $5 \%$ \\
\hline Pressure Control Valve & $\begin{array}{l}\text { PV101-10V, Omega Inc., } \\
\text { Laval, Quebec, Canada }\end{array}$ & $\begin{array}{c}-10-50^{\circ} \mathrm{C} \\
200 \mathrm{psig}(\max )\end{array}$ & $0.5 \%$ \\
\hline Heating Tape & $\begin{array}{l}\text { Heat tape with the controller, } \\
\text { HTWC 101, Omega, Inc., } \\
\text { Laval, Quebec, Canada }\end{array}$ & $232^{\circ} \mathrm{C}(\max )$ & $5 \%$ \\
\hline Digital thermometer & $\begin{array}{c}\text { VWR traceable digital } \\
\text { thermometer with recorder } \\
\text { output, Mississauga, Ontario, } \\
\text { Canada }\end{array}$ & $-50-1300^{\circ} \mathrm{C}$ & $0.3 \%$ \\
\hline Viscometer & $\begin{array}{c}\text { VISCO PRO 2000, } \\
\text { Cambridge Viscosity, Inc., } \\
\text { Medford, MA, USA }\end{array}$ & $0.2-20,000 \mathrm{cP}$ & $\begin{array}{l}1.0 \% \text { (of full } \\
\text { scale) }\end{array}$ \\
\hline $\begin{array}{c}\text { Differential Pressure } \\
\text { Transducer }\end{array}$ & $\begin{array}{l}\text { differential pressure } \\
\text { transducer, PX409, Omega, } \\
\text { Inc., Laval, Quebec, Canada }\end{array}$ & $\begin{array}{l}-45-121^{\circ} \mathrm{C} \\
0-200(\mathrm{psig})\end{array}$ & $0.08 \%$ \\
\hline $\begin{array}{l}\text { Gas Chromatography } \\
\text { Mass Spectrometry } \\
\text { with a thermal } \\
\text { conductivity detector }\end{array}$ & $\begin{array}{c}\text { Perkin Elmer Autosystem XL } \\
\text { GCMS/TCD, Woodbridge, } \\
\text { Ontario, Canada }\end{array}$ & $1.0-1200 \mathrm{u}(\mathrm{amu})$ & $\begin{array}{l} \pm 0.1 \mathrm{~m} / \mathrm{z} \\
\text { mass } \\
\text { accuracy }\end{array}$ \\
\hline
\end{tabular}




\section{Chapter 4: Optimal Control of Air Injection}

This chapter presents the optimal control of air injection gas EOR to maximize heavy oil recovery using air interfacial temperature versus time as a control function. For this purpose, the necessary conditions for maximum heavy oil recovery are derived based on detailed heat and mass transfer model of the gas EOR experiments carried out in this study. The model enables the determination of oil production, and the change in the height of the physical model with time. A computational algorithm is developed to compute the optimal policy.

\subsection{Determination of Optimal Control}

Optimal control principles help us to determine the policies that optimize some performance criterion, subject to the constraints imposed by the physical nature of the underlying process. The principles are based on the classical calculus of variations. Even for modest processes, the application of optimal control principles generates highly nonlinear differential equations that require the use of numerical techniques for the solution. ${ }^{48}$

In this study, we use the interfacial air temperature versus time as the control function. The determination of optimal control is based on the mathematical model of the experimental process. It is important to note that the model comprising of mass and heat transfer balances is a set of highly non-linear partial differential equations, and poses a moving boundary problem. Its analytical solution is not possible. Therefore, the optimal control problem is solved numerically

after deriving and satisfying the necessary conditions for optimal control. ${ }^{48}$ A computational algorithm is implemented in $\mathrm{C}++$ language is used to solve the optimal control problem. 


\subsection{Mathematical Model Formulation}

As mentioned earlier, we develop a detailed mass and heat transfer models of the air injection process for heavy oil recovery based on previous studies. The assimilation of heat transfer is done for the first time in this work. The details are as follows.

\subsubsection{Mass Transfer Model}

A mathematical model below is to describe the mass transfer process in the experiments mentioned above. The assumptions of the model developed are as follows:

1. The flow of the live oil along the vertical direction is governed by Darcy's law in the porous medium of the lab-scale heavy oil reservoirs used in this work

Justification: The live oil flow is a creeping flow since Reynold's numbers (Re) calculated for the live oil flow are less than unity.

2. The diffusion of the air takes place along the radial direction only. diffusion in the vertical direction is neglected.

Justification: Diffusion occurs in the radial direction over a large surface area along the circumference of the cylindrical model. In contrast, diffusion in the vertical direction occurs over a much smaller area at the top of the cylindrical model. Moreover, convection is dominant in the vertical direction.

3. The porous medium has uniform porosity and permeability

Justification: The oil was mixed uniformly with glass beads and packed in a cylindrical stainless steel wire mesh. Thus, each model has a uniform permeability, and no heterogeneities exist (please see Section 3.3).

4. The density of the live oil is assumed constant. 
Justification: A sensitivity analysis (see Section 5.2.7) shows that the small variation $( \pm 5 \%)$ in the live oil density $(\rho)$, the change in maximum objective functional in comparison to base value at 204 Darcy permeability and air pressure of $0.514 \mathrm{MPa}$ (absolute) is insignificant.

5. There were no chemical reactions as the absorption of the solvent gas in heavy oil is purely a physical phenomenon. (please see Section 5.1.5)

The unsteady state mass balance for the air in a cylindrical differential element is given by:

$\left(\begin{array}{c}\text { Accumulation of the } \\ \text { solvent mass over a } \\ \text { finite time interval } \Delta \mathrm{t}\end{array}\right)=\left(\begin{array}{c}\text { Rate of the solvent } \\ \text { mass input along } \\ r \text { and } z \\ \text { direction }\end{array}\right)-\left(\begin{array}{c}\text { Rate of the solvent } \\ \text { mass output along } \\ \mathrm{r} \text { and z direction }\end{array}\right)$

As per our assumption, the penetration of the air takes along r-direction. The transport of the air along r-direction is given by Fick's first law:

$\left(\begin{array}{c}\text { Rate of the solvent } \\ \text { mass input along } \\ r \\ \text { direction }\end{array}\right)-\left(\begin{array}{c}\text { Rate of the solvent } \\ \text { mass output along } \\ r \\ \text { direction }\end{array}\right)=\left(J_{g} S\right)_{r}-\left(J_{g} S\right)_{r+\Delta r}$

where $J_{g}$ is the mass flux of the air, and is given by:

$J_{g}=-\rho D \frac{\partial \omega}{\partial r}$

The mass transfer of the live oil along z-direction is governed by the Darcy flow. $D$ is undermined air dispersion in the porous media which is a function of both concentration and temperature. The dispersion of the solvent gas along $\mathrm{z}$-direction is negligible while bulk is moving: 


$$
\left(\begin{array}{c}
\text { Rate of the solvent } \\
\text { mass input along } \\
\mathrm{z} \\
\text { direction }
\end{array}\right)-\left(\begin{array}{c}
\text { Rate of the solvent } \\
\text { mass output along } \\
\mathrm{z} \\
\text { direction }
\end{array}\right)=(v A \rho \omega)_{z}-(v A \rho \omega)_{z+\Delta z}
$$

Substituting Equations (16) and (18) into (15) results in the following unsteady state mass balance for the solvent gas equation

$\frac{\delta}{\delta t}(V \varnothing \rho \omega)=\left[(v A \rho \omega)_{z}+\left(J_{g} S\right)_{r}\right]-\left[(v A \rho \omega)_{z+\Delta z}+\left(J_{g} S\right)_{r+\Delta r}\right]$

In the equation above, $\varnothing$ is the medium porosity, $V$ is the finite differential volume, $S$ is the finite differential surface area along r-direction, and $\mathrm{A}$ is the finite differential cross-sectional area along z-direction are given by:

$V=2 \pi r \Delta r \Delta z$

$S=2 \pi r \Delta z$

$A=2 \pi r \Delta r$

By substituting the Equations (17), (20), (21), and (22) into Equation (19) yields the following unsteady state partial differential equation.:

$\frac{\partial \omega}{\partial t}=\frac{\partial D}{\partial \omega}\left(\frac{\partial \omega}{\partial r}\right)^{2}+D\left[\frac{1}{r} \frac{\partial \omega}{\partial r}+\frac{\partial^{2} \omega}{\partial r^{2}}\right]-\frac{1}{\varnothing}\left[\omega \frac{\partial v}{\partial \omega} \cdot \frac{\partial \omega}{\partial z}+v \frac{\partial \omega}{\partial z}\right]$

In the above equation, $\omega$ is the mass fraction of air and $v$ is the Darcy velocity of the live oil within porous media along z-direction given by: 
$v=\frac{K_{r} K \rho g}{\mu}$

where $\mu$ is the viscosity of live oil, $\rho$ is the density of the live oil, $K_{r}$ is the relative permeability, $K$ is the medium permeability and $g$ is gravity. Since the porous medium is saturated only with heavy oil, the effective permeability equals the absolute permeability, and then the relative permeability is equal to unity.

The following is the correlation used for $\mu:^{49}$

$\mu=\mu_{o} \omega^{-2}$

where $\mu_{o}$ is the live oil viscosity coefficient, calculated from experimentally obtained live oil viscosity and solvent gas solubility mass fraction.

By putting the above expressions in Equation (23), the mass balance equation over finite element can be written as:

$\frac{\partial \omega}{\partial t}=\left(\frac{\partial D}{\partial \omega}\right) \cdot\left(\frac{\partial \omega}{\partial r}\right)^{2}+\frac{D}{r}\left(\frac{\partial \omega}{\partial r}\right)+D\left(\frac{\partial^{2} \omega}{\partial r^{2}}\right)-3 \alpha \frac{\omega^{2}}{\phi}\left(\frac{\partial \omega}{\partial z}\right)$

where $\alpha=\frac{K K_{r} \rho g}{\mu_{o}}$

$\underline{\text { Initial and Boundary Conditions for the Mass Transfer }}$

Initially, there was no air inside the packing. However, at all time, its surface has the air concentration equal to its equilibrium saturation concentration under prevailing temperature and pressure. There is no production of live oil at the beginning, so the initial height of the physical model of the heavy oil sample is $Z_{o}$. 
Thus the initial conditions at $\mathrm{t}=0$ are:

$$
\begin{aligned}
& \omega=\left\{\begin{array}{cl}
0, & \forall 0<z<Z_{o}, \forall 0 \leq r<R \\
\omega_{\text {int }}(0), & \text { at } z \in\left\{0, Z_{0}\right\} \forall 0 \leq r<R ; \text { and at } r=R \text { and } 0 \leq z \leq Z_{o}
\end{array}\right. \\
& \mathrm{Z}=\mathrm{Z}_{0} \forall 0 \leq r \leq R
\end{aligned}
$$

It is important to note that $r$ is the radius measured radially outward from the center vertical axis of the physical model and $z$ is the vertical distance measured upward from the bottom of the model as it shown in Figure 5.

Furthermore, the boundary conditions at $t \geq 0$ are:

$\omega=\omega_{\text {int }}(t)$ at $r=R \quad \forall 0 \leq z \leq Z(r, t)$ and $z \in\{0, Z(r, t)\} \forall 0 \leq r<R$

where $Z(r, t)$ is the height of the heavy oil in the physical model at a given $r$ and $t$. Furthermore, due to symmetric of the physical model, we have the following:

$\frac{\partial \omega}{\partial r}=0 \forall 0 \leq z \leq Z(0, t)$ and $r=0$ 


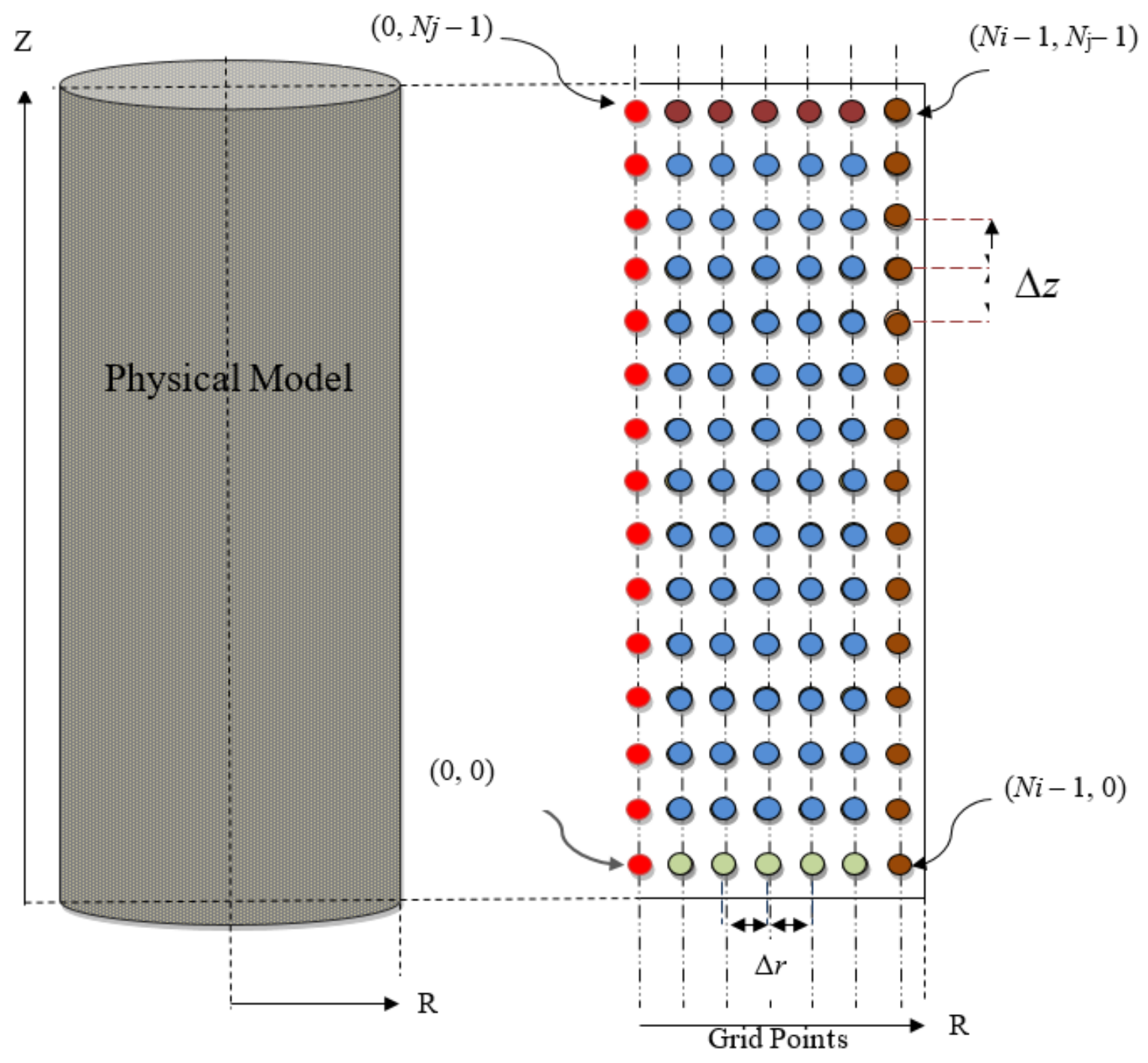

- Grid Points at Axis

Intermediate Grid Points at the Lower Boundary

Right most Grid Points

Intermediate Grid Points

Intermediate Grid Points at the Upper Boundary

Figure 5: Cylindrical Model and Node Distribution 


\subsubsection{Change in Height at Any Time}

The production of oil reduces the height $Z(t, r)$ of the bitumen in the packed medium. The change in the height with time at any radial location is given by the negative of Darcy velocity at the bottom of the physical model as:

$\frac{\partial Z}{\partial t}=\frac{-v_{o}(t, r, 0)}{\emptyset}=-\frac{K_{r} K \rho g}{\mu \emptyset}$

where $v_{o}(t, r, 0)$ is the Darcy velocity of the produced oil at a given $r$, and averaged over the differential volume, $2 \pi \mathrm{rdrdz}$ at the bottom, that is, at $Z=0$. It is important to note that The solventheavy oil interface at the top of the physical model moves down and the height of the bitumen, $Z(t, r)$, decreases with time due to live oil drainage. Thus, we have a moving boundary problem, which is described by Equation (30).

\subsubsection{Calculation Mass of Live Oil}

The cumulative mass of produced live oil at any time was calculated from multiplying differential volume with live oil density and medium porosity. For a calculated change in height mass of live oil produced was obtained by integrating the multiple from 0 to $R$ as:

$m_{c a l}=2 \pi \rho \emptyset \int_{0}^{R}\left(Z_{o}-Z(r, t)\right) r d r$

\subsubsection{Heat Transfer Model}

The mathematical below is for the heat transfer process for the porous media in the experiments as mentioned earlier. The assumptions of the model developed are as follows:

1. The flow of the live oil along the vertical direction is governed by Darcy's law in a porous medium; Negligible radioactive effects. 
Justification: The live oil flow is a creeping flow since Reynold's numbers (Re) calculated for the live oil flow are less than unity.

2. The porous medium has uniform porosity and permeability.

Justification: As previously mentioned, the oil was mixed uniformly with glass beads and packed in a cylindrical stainless steel wire mesh. Thus, each model has a uniform permeability, and no heterogeneities exist.

3. The density of the live oil is assumed constant.

Justification: As previously mentioned, sensitivity analysis shows that the small variation $( \pm 5 \%)$ in the live oil density $(\rho)$, the change in the maximum objective functional in comparison to base value at 204 Darcy permeability, and air pressure of $0.514 \mathrm{MPa}$ (absolute) is insignificant.

4. There are no chemical reactions in the process (please see Section 5.1.5).

5. The specific heat capacity $\left(C_{\mathrm{p}}\right)$ and the thermal conductivity coefficient $(k)$ are assumed constant.

Justification: Sensitivity analyses (see Section 5.2.7) show that the small variation ( \pm $5 \%)$ in the specific heat capacity $\left(C_{\mathrm{p}}\right)$ and the thermal conductivity coefficient $(k)$, the percentage changes in maximum objective functional in comparison to base value at 204 Darcy permeability and air pressure of $0.514 \mathrm{MPa}$ (absolute) are insignificant.

Similar to the mass transfer model, the heat transfer model of the lab-scale reservoir involving heat conduction is given by Equation (32)

$\emptyset\left(\rho C_{p}\right) \frac{\partial T}{\partial t}+\rho C_{p}\left(v \frac{\partial T}{\partial z}\right)=\varnothing k\left[\frac{1}{r} \frac{\partial}{\partial r}\left(r \frac{\partial T}{\partial r}\right)+\frac{\partial^{2} T}{\partial z^{2}}\right]$

where $C_{\mathrm{p}}$ is the specific heat capacity and $k$ is the thermal conductivity coefficient. 
Simplification the equation above, we finally obtain:

$\frac{\partial T}{\partial t}=\left(\frac{k}{\rho C_{p}}\right)\left[\frac{1}{r} \frac{\partial}{\partial r}\left(r \frac{\partial T}{\partial r}\right)+\frac{\partial^{2} T}{\partial z^{2}}\right]-\frac{v}{\varnothing} \frac{\partial T}{\partial z}$

or

$\frac{\partial T}{\partial t}=\gamma\left[\frac{1}{r} \frac{\partial T}{\partial r}+\frac{\partial^{2} T}{\partial r^{2}}\right]+\gamma\left[\frac{\partial^{2} T}{\partial z^{2}}\right]-\alpha \frac{\omega^{2}}{\emptyset}\left[\frac{\partial T}{\partial z}\right]$

by letting $\gamma=\frac{k}{\rho C_{p}}$

\section{$\underline{\text { Initial and Boundary Conditions for the Heat Transfer }}$}

Similar to mass transfer, we are required to apply initial and boundary conditions for heat transfer equation. The initial conditions at $t=0$ are as follows:

$T=\left\{\begin{array}{cl}0, & \forall 0<z<Z_{o}, \forall 0 \leq r<R \\ T_{\text {int }}, & \text { at } z \in\left\{0, Z_{0}\right\} \forall 0 \leq r<R ; \text { and at } r=R \text { and } 0 \leq z \leq Z_{o}\end{array}\right.$

In more detail, at the initial time, there is no air inside the packing. Although, the physical model surface has the interfacial temperature equal to its equilibrium interfacial temperature under prevailing temperature and pressure. Lastly, due to the fact that there is no production of live oil at the beginning. The initial height of the physical model of the heavy oil sample is $Z_{o}$.

The boundary conditions at $\mathrm{t} \geq 0$ are

$T=T_{\text {int }}$ at $r=R \quad \forall 0 \leq z \leq Z(r, t)$ and $z \in\{0, Z(r, t)\} \forall 0 \leq r<R$

Furthermore, due to the symmetry of the physical model:

$\frac{\partial T}{\partial r}=0 \quad \forall 0 \leq z \leq Z(0, t)$ and $r=0$ 
In the next step, an optimal control problem will be formulated to determine the desired interfacial temperature versus time $\left[T_{\mathrm{int}}(t)\right]$ function that minimizes the difference between the modelpredicted and the experimentally measured values of the cumulative live oil produced.

The role of interfacial temperature is significant in gas EOR, particularly since air mass fraction dissolve inside heavy oil media is a strong function of temperature $\left[\omega_{\text {int }}=\omega_{\text {int }}\left(T_{\text {int }}\right)\right]$.

\subsection{Formulation of Optimal Control Problem}

The objective of this problem is to maximize the heavy oil recovery using atmospheric air by using the interfacial temperature versus time as the control function. The latter translates to $T_{\text {int }}$ versus time in the optimal control problem as follows

Mathematically, the problem is to maximize objective functional $I$ which is written as:

$I=\int_{0}^{t_{f}} m d t$

where, $m$ is the cumulative mass of produced live oil and $t_{f}$ is the duration of the oil recovery experiment. With the help of Equation (31), the objective functional becomes;

$I=\beta \int_{0}^{t_{f}} \int_{0}^{R}\left(Z_{o}-Z(r, t)\right) r d r d t$

where $\beta=2 \pi \rho \emptyset$.

The maximum of $I$ is subject to Equations (26), (30), and (34), which can be expressed as, respectively: 
$G_{1}(t, r, z)=-\frac{\partial T}{\partial t}+f_{1}=0$

where

$f_{1}=\gamma\left[\frac{1}{r} \frac{\partial T}{\partial r}+\frac{\partial^{2} T}{\partial r^{2}}\right]+\gamma\left[\frac{\partial^{2} T}{\partial z^{2}}\right]-\alpha \frac{\omega^{2}}{\emptyset}\left[\frac{\partial T}{\partial z}\right]$

Furthermore, we have:

$G_{2}(t, r, z)=-\frac{\partial \omega}{\partial t}+f_{2}=0$

where

$f_{2}=\left(\frac{\partial D}{\partial \omega}\right) \cdot\left(\frac{\partial \omega}{\partial r}\right)^{2}+\frac{D}{r}\left(\frac{\partial \omega}{\partial r}\right)+D\left(\frac{\partial^{2} \omega}{\partial r^{2}}\right)-3 \alpha \frac{\omega^{2}}{\phi}\left(\frac{\partial \omega}{\partial z}\right)$

Lastly, we have:

$H(t, r)=-\frac{\partial Z}{\partial t}+f_{3}=0$

where,

$f_{3}=\frac{-v(t, r, 0)}{\emptyset}=\frac{-\alpha[\omega(t, r, 0)]^{2}}{\emptyset}$

The above equation was determined by substituting Equation (25) into Equation (24) for viscosity, and also knowing that $\alpha=\frac{K K_{r} \rho g}{\mu_{o}}$.

Equations (40), (42), and (44) are the constraints for the objective functional $I$. These constraints are highly non-linear partial differential equations. Therefore, to solve the optimal control problem, three unterminated costate variables $\lambda_{1}(t, r, z), \lambda_{2}(t, r, z)$, and $\lambda_{3}(t, r)$ are introduced into Equation (40) to obtain the following augmented objective functional: 
$J=I+\int_{0}^{t_{f}} \int_{0}^{R} \int_{0}^{Z} \lambda_{1}(t, r, z) G_{1}(t, r, z) d z d r d t+\int_{0}^{t_{f}} \int_{0}^{R} \int_{0}^{Z} \lambda_{2}(t, r, z) G_{2}(t, r, z) d z d r d t+$

$\int_{0}^{t_{f}} \int_{0}^{R} \lambda_{3}(t, r) H(t, r) d r d t$

It is important to note that in the equation above, Lagrange multipliers (sometimes called adjoint or costate variables) are used. Costate variables are used to ensure that constraints given by the equations of the simulation model are satisfied. The costate variables $\lambda_{1}(t, r, z), \lambda_{2}(t, r, z)$, and $\lambda_{3}(t, r)$ are introduced to form the augmented functional by adjoining the constraining relations to $I$. Note that for any $\lambda_{1}, \lambda_{2}$, and $\lambda_{3}, J=I$ if the constraints are satisfied.

The Lagrange multiplier corresponding to the heat transfer model (i.e., the constraint defined by the partial differential equation for the change in temperature) is the change in the augmented objective functional due to the relaxation in the constraint. ${ }^{47}$ Other Lagrange multipliers can be similarly interpreted.

By substituting Equations (39), (40), (42), and (44) into Equation (46), we get:

$J=\beta \int_{0}^{t_{f}} \int_{0}^{R}\left(Z_{o}-Z\right) r d r d t+\int_{0}^{t_{f}} \int_{0}^{R} \int_{0}^{Z} \lambda_{1}\left[-\frac{\partial T}{\partial t}+\gamma\left[\frac{1}{r} \frac{\partial T}{\partial r}+\frac{\partial^{2} T}{\partial r^{2}}\right]+\gamma\left[\frac{\partial^{2} T}{\partial z^{2}}\right]-\right.$

$\left.\alpha \frac{\omega^{2}}{\emptyset}\left[\frac{\partial T}{\partial z}\right]\right] d z d r d t+\int_{0}^{t_{f}} \int_{0}^{R} \int_{0}^{Z} \lambda_{2}\left[-\frac{\partial \omega}{\partial t}+\left(\frac{\partial D}{\partial \omega}\right) \cdot\left(\frac{\partial \omega}{\partial r}\right)^{2}+\frac{D}{r}\left(\frac{\partial \omega}{\partial r}\right)+D\left(\frac{\partial^{2} \omega}{\partial r^{2}}\right)-\right.$

$\left.3 \alpha \frac{\omega^{2}}{\phi}\left(\frac{\partial \omega}{\partial z}\right)\right] d z d r d t+\int_{0}^{t_{f}} \int_{0}^{R} \lambda_{3}\left[-\frac{\partial z}{\partial t}+f_{3}\right] d r d t$

where $\lambda_{1}(t, r, z), \lambda_{2}(t, r, z)$, and $\lambda_{3}(t, r)$ are the costate variables. The maximum of $I$ subjected to constraints is equivalent to the maximization of $J$. 


\subsection{Necessary Conditions for the Optimal Control}

The necessary conditions for the optimum of $J$ stem from $\delta J=0$. Therefore, we can proceed it by deriving the expression for $\delta J$ as follows:

$\delta J=\delta I+\int_{0}^{t_{f}} \int_{0}^{R} \int_{0}^{Z}\left(G_{1} \delta \lambda_{1}+\lambda_{1} \delta G_{1}\right) d z d r d t+\int_{0}^{t_{f}} \int_{0}^{R} \int_{0}^{Z}\left(G_{2} \delta \lambda_{2}+\lambda_{2} \delta G_{2}\right) d z d r d t+$

$\int_{0}^{t_{f}} \int_{0}^{R}\left(H \delta \lambda_{3}+\lambda_{2} \delta H\right) d r d t$

By expansion of $\delta G_{1}, \delta G_{2}$, and $\delta H$ in the Equation above, it leads to:

$\delta J=-\beta \int_{0}^{t_{f}} \int_{0}^{R} r \delta Z d r d t-\int_{0}^{t_{f}} \int_{0}^{R} \int_{0}^{Z} \lambda_{1} \frac{\partial(\delta T)}{\partial t} d z d r d t+\int_{0}^{t_{f}} \int_{0}^{R} \int_{0}^{Z} \lambda_{1} \frac{\partial\left(f_{1}\right)}{\partial T} \delta T d z d r d t+$

$\int_{0}^{t_{f}} \int_{0}^{R} \int_{0}^{Z} \lambda_{1} \frac{\partial\left(f_{1}\right)}{\partial T_{r}} \delta T_{r} d z d r d t+\int_{0}^{t_{f}} \int_{0}^{R} \int_{0}^{Z} \lambda_{1} \frac{\partial\left(f_{1}\right)}{\partial T_{r r}} \delta T_{r r} d z d r d t+\int_{0}^{t_{f}} \int_{0}^{R} \int_{0}^{Z} \lambda_{1} \frac{\partial\left(f_{1}\right)}{\partial T_{z}} \delta T_{z} d z d r d t+$

$\int_{0}^{t_{f}} \int_{0}^{R} \int_{0}^{Z} \lambda_{1} \frac{\partial\left(f_{1}\right)}{\partial T_{z z}} \delta T_{z z} d z d r d t-\int_{0}^{t_{f}} \int_{0}^{R} \int_{0}^{Z} \lambda_{2} \frac{\partial(\delta \omega)}{\partial t} d z d r d t+\int_{0}^{t_{f}} \int_{0}^{R} \int_{0}^{Z} \lambda_{2} \frac{\partial\left(f_{2}\right)}{\partial \omega} \delta \omega d z d r d t+$

$\int_{0}^{t_{f}} \int_{0}^{R} \int_{0}^{Z} \lambda_{2} \frac{\partial\left(f_{2}\right)}{\partial \omega_{z}} \delta \omega_{z} d z d r d t+\int_{0}^{t_{f}} \int_{0}^{R} \int_{0}^{Z} \lambda_{2} \frac{\partial\left(f_{2}\right)}{\partial \omega_{r}} \delta \omega_{r} d z d r d t+\int_{0}^{t_{f}} \int_{0}^{R} \int_{0}^{Z} \lambda_{2} \frac{\partial\left(f_{2}\right)}{\partial \omega_{r r}} \delta \omega_{r r} d z d r d t-$

$\int_{0}^{t_{f}} \int_{0}^{R} \lambda_{3} \frac{\partial(\delta Z)}{\partial t} d r d t+\int_{0}^{t_{f}} \int_{0}^{R} \lambda_{3} \delta f_{3} d r d t+\int_{0}^{t_{f}} \int_{0}^{R} \int_{0}^{Z} G_{1} \delta \lambda_{1} d z d r d t+$

$\int_{0}^{t_{f}} \int_{0}^{R} \int_{0}^{Z} G_{2} \delta \lambda_{2} d z d r d t+\int_{0}^{t_{f}} \int_{0}^{R} H \delta \lambda_{3} d r d t=0$

where

$T_{r}=\frac{\partial T}{\partial r}, T_{z}=\frac{\partial T}{\partial z}, T_{r r}=\frac{\partial^{2} T}{\partial r^{2}}, T_{z z}=\frac{\partial^{2} T}{\partial z^{2}}$

and

$\omega_{r}=\frac{\partial \omega}{\partial r}, \omega_{z}=\frac{\partial \omega}{\partial z}, \omega_{r r}=\frac{\partial^{2} \omega}{\partial r^{2}}$ 


\section{Simplification of $\delta J$ using Integration by Parts}

Applying integration by parts of the second integral of Equation (49), it leads to:

$\int_{0}^{t_{f}} \int_{0}^{R} \int_{0}^{Z} \lambda_{1} \frac{\partial(\delta T)}{\partial t} d z d r d t=\left.\int_{0}^{R} \int_{0}^{Z} \lambda_{1} \delta T\right|_{0} ^{t_{f}} d z d r-\int_{0}^{t_{f}} \int_{0}^{R} \int_{0}^{Z} \frac{\partial \lambda_{1}}{\partial t} \delta T d z d r d t$

By applying the initial conditions into the first integral on the right hand side in Equation (52). Because the initial temperature of solvent is fixed, $\delta T(0, r, z)$ is zero for all $r$ and $z$. Now, if we specify:

$$
\lambda_{1}\left(t_{f}, r, z\right)=0,0 \leq r \leq R \text { and } 0 \leq z \leq Z_{0}
$$

Therefore, Equation (52) can be written as:

$\int_{0}^{t_{f}} \int_{0}^{R} \int_{0}^{Z} \lambda_{1} \frac{\partial(\delta T)}{\partial t} d z d r d t=-\int_{0}^{t_{f}} \int_{0}^{R} \int_{0}^{Z} \frac{\partial \lambda_{1}}{\partial t} \delta T d z d r d t$

By applying then boundary conditions into the fourth part of the integral Equation (49), we can obtain the following equation:

$\int_{0}^{t_{f}} \int_{0}^{R} \int_{0}^{Z} \lambda_{1}\left(\frac{\partial f_{1}}{\partial T_{r}} \delta T_{r}\right) d z d r d t=\left.\int_{0}^{t_{f}} \int_{0}^{Z} \lambda_{1} \frac{\partial f_{1}}{\partial T_{r}} \delta T\right|_{0} ^{R} d z d t-\int_{0}^{t_{f}} \int_{0}^{R} \int_{0}^{Z} \frac{\partial}{\partial r}\left(\lambda_{1} \frac{\partial f_{1}}{\partial T_{r}} \delta T\right) d z d r d t$

Considering that $\delta T$ is arbitrary at $r=0$ and the fact that all additive terms of $\delta J$ must be individually zero, it follows that:

$$
\lambda_{1}(t, 0, z)=0 \forall t: 0 \leq t \leq t_{f} \text { and } 0 \leq z \leq Z
$$


Which it yields to:

$\int_{0}^{t_{f}} \int_{0}^{R} \int_{0}^{Z} \lambda_{1}\left(\frac{\partial f_{1}}{\partial T_{r}} \delta T_{r}\right) d z d r d t=\int_{0}^{t_{f}} \int_{0}^{Z} \lambda_{1} \frac{\partial f_{1}}{\partial T_{r}} \delta T(t, R, z) d z d t-$

$\int_{0}^{t_{f}} \int_{0}^{R} \int_{0}^{Z} \frac{\partial}{\partial r}\left(\lambda_{1} \frac{\partial f_{1}}{\partial T_{r}} \delta T\right) d z d r d t$

Applying integration by part to the fifth integral of Equation (49), we have obtained the following equation:

$\int_{0}^{t_{f}} \int_{0}^{R} \int_{0}^{Z} \lambda_{1}\left(\frac{\partial f_{1}}{\partial T_{r r}} \delta T_{r r}\right) d z d r d t=\left.\int_{0}^{t_{f}} \int_{0}^{Z} \lambda_{1} \frac{\partial f_{1}}{\partial T_{r r}} \delta T_{r}\right|_{0} ^{R} d z d t-$

$\int_{0}^{t_{f}} \int_{0}^{R} \int_{0}^{Z} \frac{\partial}{\partial r}\left(\lambda_{1} \frac{\partial f_{1}}{\partial T_{r r}}\right) \delta T_{r} d z d r d t$

By applying integration by part to the second integral of Equation (56) with respect to r-results in:

$\int_{0}^{t_{f}} \int_{0}^{R} \int_{0}^{Z} \lambda_{1}\left(\frac{\partial f_{1}}{\partial T_{r r}}\right) \delta T_{r} d z d r d t=\left.\int_{0}^{t_{f}} \int_{0}^{Z} \lambda_{1} \frac{\partial f_{1}}{\partial T_{r r}} \delta T_{r}\right|_{0} ^{R} d z d t-\left.\int_{0}^{t_{f}} \int_{0}^{Z} \frac{\partial}{\partial r}\left(\lambda_{1} \frac{\delta f_{1}}{\delta T_{r r}}\right) \delta T\right|_{0} ^{R} d z d t+$

$\int_{0}^{t_{f}} \int_{0}^{R} \int_{0}^{Z} \frac{\partial^{2}}{\partial r^{2}}\left(\lambda_{1} \frac{\partial f_{1}}{\partial T_{r r}}\right) \delta T d z d r d t$

Considering that $\delta T$ is arbitrary at $r=R$ and the fact that all additive terms of $\delta J$ must be individually zero, it follows that

$\lambda_{1}(t, R, z)=0,0 \leq t \leq t_{f}, 0 \leq z \leq Z$

In addition, that $T_{r}$ is zero at $r=0$ because of the symmetric geometry of the physical model around the z-axis. This makes Equation (56) to be simplified further as:

$\int_{0}^{t_{f}} \int_{0}^{R} \int_{0}^{Z} \lambda_{1}\left(\frac{\partial f_{1}}{\partial T_{r r}} \delta T_{r r}\right) d z d r d t=-\int_{0}^{t_{f}} \int_{0}^{Z} \frac{\partial \lambda_{1}}{\partial r}\left(\frac{\partial f_{1}}{\partial T_{r r}}\right) \partial T_{r}(t, R, z) d z d t+$

$\int_{0}^{t_{f}} \int_{0}^{R} \int_{0}^{Z} \frac{\partial^{2}}{\partial r^{2}}\left(\lambda_{1} \frac{\partial f_{1}}{\partial T_{r r}}\right) \delta T d z d r d t$

Applying integration by parts of the sixth integral of Equation (49), we obtain: 
$\int_{0}^{t_{f}} \int_{0}^{R} \int_{0}^{Z} \lambda_{1} \frac{\partial f_{1}}{\partial T_{z}} \delta T_{z} d z d r d t=\left.\int_{0}^{t_{f}} \int_{0}^{R} \lambda_{1} \frac{\partial f_{1}}{\partial T_{z}} \delta T_{z}\right|_{0} ^{Z} d r d t-\int_{0}^{t_{f}} \int_{0}^{R} \int_{0}^{Z} \frac{\partial}{\partial z}\left(\lambda_{1} \frac{\partial f_{1}}{\partial T_{z}}\right) \partial T_{z} d z d r d t$

By applying the boundary conditions into the first integral of Equation (60) we obtain the following equation:

$$
\begin{aligned}
& \left(-\left.\lambda_{1} \frac{\partial f_{1}}{\partial T_{z}} \delta T\right|_{Z}+\left.\lambda_{1} \frac{\partial f_{1}}{\partial T_{z}} \delta T\right|_{0}\right) d z d t \\
& T=T(t) \text { at } z=0 \text { and } z=Z(r, t) \forall 0 \leq r<R
\end{aligned}
$$

Therefore, Equation (61) becomes:

$\int_{0}^{t_{f}} \int_{0}^{R} \int_{0}^{Z} \lambda_{1} \frac{\partial f_{1}}{\partial T_{z}} \delta T_{z} d z d r d t=\int_{0}^{t_{f}} \int_{0}^{R} \lambda_{1} \frac{\partial f_{1}}{\partial T_{z}} \delta T(t, r, Z) d r d t-$

$\int_{0}^{t_{f}} \int_{0}^{R} \lambda_{1} \frac{\partial f_{1}}{\partial T_{z}} \delta T(t, r, 0) d r d t-\int_{0}^{t_{f}} \int_{0}^{R} \int_{0}^{Z} \frac{\partial}{\partial z}\left(\lambda_{1} \frac{\delta f_{1}}{\delta T_{z}}\right) \partial T d z d r d t$

Applying integration by part of the seventh integral of Equation (49):

$\int_{0}^{t_{f}} \int_{0}^{R} \int_{0}^{Z} \lambda_{1} \frac{\partial f_{1}}{\partial T_{z z}} \delta T_{z z} d z d r d t=\left.\int_{0}^{t_{f}} \int_{0}^{R} \lambda_{1} \frac{\partial f_{1}}{\partial T_{z z}} \delta T\right|_{0} ^{Z} d r d t-\int_{0}^{t_{f}} \int_{0}^{R} \int_{0}^{Z} \frac{\partial}{\partial z}\left(\lambda_{1} \frac{\partial f_{1}}{\partial T_{z Z}}\right) \partial T_{z} d z d r d t$

Applying the integration by part to the second integral of Equation (63):

$$
\begin{aligned}
& \int_{0}^{t_{f}} \int_{0}^{R} \int_{0}^{Z} \lambda_{1}\left(\frac{\partial f_{1}}{\partial T_{z Z}}\right) \partial T_{z} d z d r d t=\left.\int_{0}^{t_{f}} \int_{0}^{R} \lambda_{1} \frac{\partial f_{1}}{\partial T_{z Z}} \delta T\right|_{0} ^{Z} d r d t-\left.\int_{0}^{t_{f}} \int_{0}^{R} \frac{\partial}{\partial z}\left(\lambda_{1} \frac{\partial f_{1}}{\partial T_{z Z}}\right) \delta T\right|_{0} ^{Z} d r d t+ \\
& \int_{0}^{t_{f}} \int_{0}^{R} \int_{0}^{Z} \frac{\partial^{2}}{\partial z^{2}}\left(\lambda_{1} \frac{\partial f_{1}}{\partial T_{z z}}\right) \partial T d z d r d t
\end{aligned}
$$

As a result, Equation (63) can be written as: 
$\int_{0}^{t_{f}} \int_{0}^{R} \int_{0}^{Z} \lambda_{1} \frac{\partial f_{1}}{\partial T_{z Z}} \delta T_{z Z} d z d r d t=\int_{0}^{t_{f}} \int_{0}^{R} \lambda_{1} \frac{\partial f_{1}}{\partial T_{z z}} \delta T(t, r, Z) d r d t-\int_{0}^{t_{f}} \int_{0}^{R} \lambda_{1} \frac{\partial f_{1}}{\partial T_{z Z}} \delta T(t, r, 0) d r d t-$ $\int_{0}^{t_{f}} \int_{0}^{R} \frac{\partial}{\partial z}\left(\lambda_{1} \frac{\partial f_{1}}{\partial T_{z z}}\right) \delta T(t, r, Z) d r d t+\int_{0}^{t_{f}} \int_{0}^{R} \frac{\partial}{\partial z}\left(\lambda_{1} \frac{\partial f_{1}}{\partial T_{z z}}\right) \delta T(t, r, 0) d r d t+$

$\int_{0}^{t_{f}} \int_{0}^{R} \int_{0}^{Z} \frac{\partial^{2}}{\partial z^{2}}\left(\lambda_{1} \frac{\partial f_{1}}{\partial T_{z z}}\right) \partial T d z d r d t$

Applying the eighth of integration from Equation (49):

$\int_{0}^{t_{f}} \int_{0}^{R} \int_{0}^{Z} \lambda_{2} \frac{\partial(\delta \omega)}{\partial t} d z d r d t=\left.\int_{0}^{R} \int_{0}^{Z} \lambda_{2} \delta \omega\right|_{0} ^{t_{f}} d z d r-\int_{0}^{t_{f}} \int_{0}^{R} \int_{0}^{Z} \frac{\partial \lambda_{2}}{\partial t} \delta \omega d z d r d t$

Because initial mass fraction of the solvent gas $\delta \omega(0, r, z)$ is zero for all $\mathrm{r}$ and $\mathrm{z}$. Now, if we specify:

$$
\lambda_{2}\left(t_{f}, r, z\right)=0 \quad \forall r: 0 \leq r \leq R \text { and } \quad \forall 0 \leq z \leq Z_{o}
$$

Therefore, Equation (66) becomes:

$\int_{0}^{t_{f}} \int_{0}^{R} \int_{0}^{Z} \lambda_{2} \frac{\partial(\delta \omega)}{\partial t} d z d r d t=-\int_{0}^{t_{f}} \int_{0}^{R} \int_{0}^{Z} \frac{\partial \lambda_{2}}{\partial t} \delta \omega d z d r d t$

Applying integration by parts of the tenth of Equation (49) with respect to z, we can obtain

$\int_{0}^{t_{f}} \int_{0}^{R} \int_{0}^{Z} \lambda_{2} \frac{\partial f_{2}}{\partial \omega_{z}} \delta \omega_{z} d z d r d t=\left.\int_{0}^{t_{f}} \int_{0}^{R} \lambda_{2} \frac{\partial f_{2}}{\delta \omega_{z}} \delta \omega\right|_{0} ^{Z} d r d t-\int_{0}^{t_{f}} \int_{0}^{R} \int_{0}^{Z} \frac{\partial}{\partial z}\left(\lambda_{2} \frac{\partial f_{2}}{\partial \omega_{z}}\right) \delta \omega d z d r d t$

Or, it can be simplified into:

$\int_{0}^{t_{f}} \int_{0}^{R} \int_{0}^{Z} \lambda_{2} \frac{\partial f_{2}}{\partial \omega_{z}} \delta \omega_{z} d z d r d t=\int_{0}^{t_{f}} \int_{0}^{R} \lambda_{2} \frac{\partial f_{2}}{\delta \omega_{z}} \delta \omega(t, r, Z) d r d t+\int_{0}^{t_{f}} \int_{0}^{R} \lambda_{2} \frac{\partial f_{2}}{\delta \omega_{z}} \delta \omega(t, r, 0) d r d t-$ $\int_{0}^{t_{f}} \int_{0}^{R} \int_{0}^{Z} \frac{\partial}{\partial z}\left(\lambda_{2} \frac{\partial f_{2}}{\partial \omega_{z}}\right) \delta \omega d z d r d t$

Applying integration by parts eleventh integral of Equation (49) we obtain the following equation: 
$\int_{0}^{t_{f}} \int_{0}^{R} \int_{0}^{Z} \lambda_{2}\left(\frac{\partial f_{2}}{\partial \omega_{r}} \delta \omega_{r}\right) d z d r d t=\left.\int_{0}^{t_{f}} \int_{0}^{Z} \lambda_{2} \frac{\partial f_{2}}{\delta \omega_{r}} \delta \omega\right|_{0} ^{R} d z d t-\int_{0}^{t_{f}} \int_{0}^{R} \int_{0}^{Z} \frac{\partial}{\partial r}\left(\lambda_{2} \frac{\partial f_{2}}{\partial \omega_{r}}\right) \delta \omega d z d r d t$

Considering that $\delta \omega$ is arbitrary at $r=0$ and the fact that all additive terms of $\delta J$ must be individually zero (Equation 49), it follows that

$$
\lambda_{2}(t, 0, z)=0 \quad \forall t: 0 \leq t \leq t_{f} \text { and } \forall 0 \leq z \leq Z_{o}
$$

Therefore, Equation (70) becomes:

$\int_{0}^{t_{f}} \int_{0}^{R} \int_{0}^{Z} \lambda_{2}\left(\frac{\partial f_{2}}{\partial \omega_{r}} \delta \omega_{r}\right) d z d r d t=\int_{0}^{t_{f}} \int_{0}^{Z} \lambda_{2} \frac{\partial f_{2}}{\delta \omega_{r}} \delta \omega(t, R, z) d z d t-$

$\int_{0}^{t_{f}} \int_{0}^{R} \int_{0}^{Z} \frac{\partial}{\partial r}\left(\lambda_{2} \frac{\partial f_{2}}{\partial \omega_{r}}\right) \delta \omega d z d r d t$

Applying integration by parts to the twelfth integral of Equation (49), we can obtain the following equation:

$\int_{0}^{t_{f}} \int_{0}^{R} \int_{0}^{Z} \lambda_{2}\left(\frac{\partial f_{2}}{\partial \omega_{r r}} \delta \omega_{r r}\right) d z d r d t=\left.\int_{0}^{t_{f}} \int_{0}^{Z} \lambda_{2} \frac{\partial f_{2}}{\partial \omega_{r r}} \delta \omega\right|_{0} ^{R} d z d t-$

$\int_{0}^{t_{f}} \int_{0}^{R} \int_{0}^{Z} \frac{\partial}{\partial r}\left(\lambda_{2} \frac{\partial f_{2}}{\partial \omega_{r r}}\right) \delta \omega_{r} d z d r d t$

Applying integration by parts to the second integral of Equation (72) with respect to $r$ results in:

$\int_{0}^{t_{f}} \int_{0}^{R} \int_{0}^{Z}\left(\lambda_{2} \frac{\partial f_{2}}{\partial \omega_{r r}}\right) \delta \omega_{r} d z d r d t=\left.\int_{0}^{t_{f}} \int_{0}^{Z} \lambda_{2} \frac{\partial f_{2}}{\partial \omega_{r r}} \delta \omega_{r}\right|_{0} ^{R} d z d t-\left.\int_{0}^{t_{f}} \int_{0}^{Z} \frac{\partial}{\partial r}\left(\lambda_{2} \frac{\partial f_{2}}{\partial \omega_{r r}}\right) \delta \omega\right|_{0} ^{R} d z d t+$

$\int_{0}^{t_{f}} \int_{0}^{R} \int_{0}^{Z} \frac{\partial^{2}}{\partial r^{2}}\left(\lambda_{2} \frac{\partial f_{2}}{\partial \omega_{r r}}\right) \delta \omega d z d r d t$

Considering that $\delta \omega$ is arbitrary at $r=R$ and the fact that all the additive terms of $\delta J$ must be individually zero (Equation (49)), it follows that:

$$
\lambda_{2}(t, R, z)=0,0 \leq t \leq t_{f}, 0 \leq z \leq Z
$$


In addition, $\omega_{r}$ is zero at $r=0$ because of symmetry around the z-axis of the cylindrical physical model that makes Equation (73) get simplified to:

$\int_{0}^{t_{f}} \int_{0}^{R} \int_{0}^{Z} \lambda_{2}\left(\frac{\partial f_{2}}{\partial \omega_{r r}} \delta \omega_{r r}\right) d z d r d t=-\int_{0}^{t_{f}} \int_{0}^{Z} \frac{\partial \lambda_{2}}{\partial r}\left(\frac{\partial f_{2}}{\partial \omega_{r r}}\right) \delta \omega(t, R, z) d z d t+$

$\int_{0}^{t_{f}} \int_{0}^{R} \int_{0}^{Z} \frac{\partial^{2}}{\partial r^{2}}\left(\lambda_{2} \frac{\partial f_{2}}{\partial \omega_{r r}}\right) \delta \omega d z d r d t$

Applying integration by parts to the thirteenth integral of Equation (49), we obtain

$\int_{0}^{t_{f}} \int_{0}^{R} \lambda_{3}\left(\frac{\partial(\delta z)}{\partial t}\right) d r d t=\left.\int_{0}^{R} \lambda_{3} \delta z\right|_{0} ^{t_{f}} d r-\int_{0}^{t_{f}} \int_{0}^{R} \frac{\partial \lambda_{3}}{\partial t} \delta Z d r d t$

Using the fact that $\delta Z$ is zero at $t=0$ because the initial height is specified as:

$$
\lambda_{3}\left(t_{f}, r\right)=0 \quad \forall 0 \leq r \leq R
$$

As a result, Equation (75) becomes:

$\int_{0}^{t_{f}} \int_{0}^{R} \lambda_{3}\left(\frac{\partial(\delta z)}{\partial t}\right) d r d t=-\int_{0}^{t_{f}} \int_{0}^{R} \frac{\partial \lambda_{3}}{\partial t} \delta Z d r d t$

The last integral of Equation (49):

$\int_{0}^{t_{f}} \int_{0}^{R} \lambda_{3} \delta f_{3} d r d t=\int_{0}^{t_{f}} \int_{0}^{R} \lambda_{3} \frac{\partial f_{3}}{\partial \omega} \delta \omega(t, r, 0) d r d t$

By substituting of the simplified into Equation (49) and rearrange, we obtain the final equation of variation of $\delta J$, which is written as:

$\delta J=-\int_{0}^{t_{f}} \int_{0}^{R} \beta r \delta z d r d t+\int_{0}^{t_{f}} \int_{0}^{R} \int_{0}^{Z} \frac{\partial \lambda_{1}}{\partial t} \delta T d z d r d t+\int_{0}^{t_{f}} \int_{0}^{R} \int_{0}^{Z} \lambda_{1} \frac{\partial f_{1}}{\partial T} \delta T d z d r d t+$

$\int_{0}^{R} \int_{0}^{Z} \lambda_{1} \frac{\partial f_{1}}{\partial T_{r}} \delta T(t, R, z) d z d t-\int_{0}^{t_{f}} \int_{0}^{R} \int_{0}^{Z} \frac{\delta}{\delta r}\left(\lambda_{1} \frac{\partial f_{1}}{\partial T_{r}} \delta T\right) d z d r d t-$

$\int_{0}^{t_{f}} \int_{0}^{Z} \frac{\partial \lambda_{1}}{\partial r}\left(\frac{\partial f_{1}}{\partial T_{r r}}\right) \partial T(t, R, z) d z d t+\int_{0}^{t_{f}} \int_{0}^{R} \int_{0}^{Z} \frac{\partial^{2}}{\partial r^{2}}\left(\lambda_{1} \frac{\partial f_{1}}{\partial T_{r r}}\right) \partial T d z d r d t+$ 
$\int_{0}^{t_{f}} \int_{0}^{R} \lambda_{1} \frac{\partial f_{1}}{\partial T_{z}} \delta T(t, r, Z) d r d t-$

$\int_{0}^{t_{f}} \int_{0}^{R} \lambda_{1} \frac{\partial f_{1}}{\partial T_{z}} \delta T(t, r, 0) d r d t-\int_{0}^{t_{f}} \int_{0}^{R} \int_{0}^{Z} \frac{\partial}{\partial z}\left(\lambda_{1} \frac{\partial f_{1}}{\partial T_{z}}\right) \partial T d z d r d t+$

$\int_{0}^{t_{f}} \int_{0}^{R} \lambda_{1} \frac{\partial f_{1}}{\partial T_{z z}} \delta T(t, r, Z) d r d t-\int_{0}^{t_{f}} \int_{0}^{R} \lambda_{1} \frac{\partial f_{1}}{\partial T_{z Z}} \delta T(t, r, 0) d r d t-$

$\int_{0}^{t_{f}} \int_{0}^{R} \frac{\partial}{\partial z}\left(\lambda_{1} \frac{\partial f_{1}}{\partial T_{z z}}\right) \delta T(t, r, Z) d r d t+\int_{0}^{t_{f}} \int_{0}^{R} \frac{\partial}{\partial z}\left(\lambda_{1} \frac{\partial f_{1}}{\partial T_{z z}}\right) \delta T(t, r, 0) d r d t+$

$\int_{0}^{t_{f}} \int_{0}^{R} \int_{0}^{Z} \frac{\partial^{2}}{\partial z^{2}}\left(\lambda_{1} \frac{\partial f_{1}}{\partial T_{z z}}\right) \partial T d z d r d t+\int_{0}^{t_{f}} \int_{0}^{R} \int_{0}^{Z} \frac{\partial \lambda_{2}}{\partial t} \delta \omega d z d r d t+\int_{0}^{t_{f}} \int_{0}^{R} \int_{0}^{Z} \lambda_{2} \frac{\partial f_{2}}{\partial \omega} \delta \omega d z d r d t+$

$\int_{0}^{t_{f}} \int_{0}^{R} \lambda_{2} \frac{\partial f_{2}}{\partial \omega_{z}} \delta \omega(t, r, Z) d r d t+\int_{0}^{t_{f}} \int_{0}^{R} \lambda_{2} \frac{\partial f_{2}}{\partial \omega_{z}} \delta \omega(t, r, 0) d r d t-$

$\int_{0}^{t_{f}} \int_{0}^{R} \int_{0}^{Z} \frac{\partial}{\partial z}\left(\lambda_{2} \frac{\partial f_{2}}{\partial \omega_{z}}\right) \delta \omega d z d r d t+\int_{0}^{t_{f}} \int_{0}^{Z} \lambda_{2} \frac{\partial f_{2}}{\partial \omega_{r}} \delta \omega(t, R, z) d z d t-$

$\int_{0}^{t_{f}} \int_{0}^{R} \int_{0}^{Z} \frac{\partial}{\partial r}\left(\lambda_{2} \frac{\partial f_{2}}{\partial \omega_{r}}\right) \delta \omega d z d r d t-\int_{0}^{t_{f}} \int_{0}^{Z} \frac{\partial \lambda_{2}}{\partial r}\left(\frac{\partial f_{2}}{\partial \omega_{r r}}\right) \delta \omega(t, R, z) d z d t+$

$\int_{0}^{t_{f}} \int_{0}^{R} \int_{0}^{Z} \frac{\partial^{2}}{\partial r^{2}}\left(\lambda_{2} \frac{\partial f_{2}}{\partial \omega_{r r}}\right) \delta \omega d z d r d t+\int_{0}^{t_{f}} \int_{0}^{R} \frac{\partial \lambda_{3}}{\partial t} \delta Z d r d t+\int_{0}^{t_{f}} \int_{0}^{R} \lambda_{3} \frac{\partial f_{3}}{\partial \omega} \delta \omega(t, r, 0) d r d t+$

$\int_{0}^{t_{f}} \int_{0}^{R} \int_{0}^{Z} G_{1} \delta \lambda_{1} d z d r d t+\int_{0}^{t_{f}} \int_{0}^{R} \int_{0}^{Z} G_{2} \delta \lambda_{2} d z d r d t+\int_{0}^{t_{f}} \int_{0}^{R} \int_{0}^{Z} H \delta \lambda_{3} d z d r d t=0$

Simplification of Equation (78):

$\delta J=\int_{0}^{t_{f}} \int_{0}^{R}\left[-\beta r+\frac{\partial \lambda_{3}}{\partial t}\right] \delta Z d r d t+\int_{0}^{t_{f}} \int_{0}^{R} \int_{0}^{Z}\left[\frac{\partial \lambda_{2}}{\partial t}+\lambda_{2} \frac{\partial f_{2}}{\partial \omega}-\frac{\partial}{\partial z}\left(\lambda_{2} \frac{\partial f_{2}}{\partial \omega_{z}}\right)-\frac{\partial}{\partial r}\left(\lambda_{2} \frac{\partial f_{2}}{\partial \omega_{r}}\right)+\right.$

$\left.\frac{\partial^{2}}{\partial r^{2}}\left(\lambda_{2} \frac{\partial f_{2}}{\partial \omega_{r r}}\right)\right] \delta \omega d z d r d t+\int_{0}^{t_{f}} \int_{0}^{R} \lambda_{2} \frac{\partial f_{2}}{\partial \omega_{z}} \delta \omega(t, r, Z) d r d t-\int_{0}^{t_{f}} \int_{0}^{R}\left[\lambda_{2} \frac{\partial f_{2}}{\partial \omega_{z}}-\right.$

$\left.\lambda_{3} \frac{\partial f_{3}}{\partial \omega}\right] \delta \omega(t, r, 0) d r d t+\int_{0}^{t_{f}} \int_{0}^{Z}\left[\lambda_{2} \frac{\partial f_{2}}{\partial \omega_{r}}+\frac{\partial \lambda_{2}}{\partial r}\left(\frac{\partial f_{2}}{\delta \omega_{r r}}\right)\right] \delta \omega(t, R, z) d z d t+\int_{0}^{t_{f}} \int_{0}^{R} \int_{0}^{Z}\left[+\frac{\delta \lambda_{1}}{\delta t}+\right.$

$\left.\lambda_{1} \frac{\partial f_{1}}{\partial T}-\frac{\partial}{\partial z}\left(\lambda_{1} \frac{\partial f_{1}}{\partial T_{z}}\right)+\frac{\partial^{2}}{\partial z^{2}}\left(\lambda_{1} \frac{\partial f_{1}}{\partial T_{z z}}\right)+\frac{\partial^{2}}{\partial r^{2}}\left(\lambda_{1} \frac{\partial f_{1}}{\partial T_{r r}}\right)-\frac{\partial}{\partial r}\left(\lambda_{1} \frac{\partial f_{1}}{\partial T_{r}}\right)\right] \delta T d z d r d t+\int_{0}^{t_{f}} \int_{0}^{R}\left[\lambda_{1} \frac{\partial f_{1}}{\partial T_{z}}+\right.$

$\left.\lambda_{1} \frac{\partial f_{1}}{\partial T_{z z}}-\frac{\partial}{\partial z}\left(\lambda_{1} \frac{\partial f_{1}}{\partial T_{z z}}\right)\right] \delta T(t, r, Z) d r d t-\int_{0}^{t_{f}} \int_{0}^{R}\left[\lambda_{1} \frac{\partial f_{1}}{\partial T_{z}}+\lambda_{1} \frac{\partial f_{1}}{\partial T_{z z}}-\right.$ 
$\left.\frac{\partial}{\partial z}\left(\lambda_{1} \frac{\partial f_{1}}{\partial T_{z z}}\right)\right] \delta T(t, r, 0) d r d t+\int_{0}^{t_{f}} \int_{0}^{Z}\left[\lambda_{1} \frac{\partial f_{1}}{\partial T_{r}}+\frac{\partial \lambda_{1}}{\partial r}\left(\frac{\partial f_{1}}{\partial T_{r r}}\right)\right] \delta T(t, R, z) d z d t+$

$\int_{0}^{t_{f}} \int_{0}^{R} \int_{0}^{Z} G_{1} \delta \lambda_{1} d z d r d t+\int_{0}^{t_{f}} \int_{0}^{R} \int_{0}^{Z} G_{2} \delta \lambda_{2} d z d r d t+\int_{0}^{t_{f}} \int_{0}^{R} \int_{0}^{Z} H \delta \lambda_{3} d z d r d t=0$

Note that

$\delta \omega(t, R, z)=\delta \omega(t, r, 0)=\delta \omega(t, r, Z)=\delta \omega_{\text {int }}(t)$

$\delta T(t, R, z)=\delta T(t, r, 0)=\delta T(t, r, Z)=\delta T_{\text {int }}(t)$

Lastly, since the interfacial air mass fraction is a strong function of temperature $\left(T_{\text {int }}\right)$ with time, we can write the following condition (Pleas see Section 3.5):

$\omega_{\text {int }}=\omega_{\text {int }}\left(T_{\text {int }}\right)$

which represent the functionality of interfacial concentration to temperature at $r=R$. As a result, the equation above can be written as:

$\delta \omega_{\text {int }}=\frac{\partial \omega_{\text {int }}}{\partial T_{\text {int }}} \times \delta T_{\text {int }}$

By applying Equations (80) and (81), we can re-write Equation (79) as:

$\delta J=\int_{0}^{t_{f}} \int_{0}^{R}\left[-\beta r+\frac{\partial \lambda_{3}}{\partial t}\right] \delta Z d r d t+\int_{0}^{t_{f}} \int_{0}^{R} \int_{0}^{Z}\left[\frac{\partial \lambda_{2}}{\partial t}+\lambda_{2} \frac{\partial f_{2}}{\partial \omega}-\frac{\partial}{\partial z}\left(\lambda_{2} \frac{\partial f_{2}}{\partial \omega_{z}}\right)-\frac{\partial}{\partial r}\left(\lambda_{2} \frac{\partial f_{2}}{\partial \omega_{r}}\right)+\right.$

$\left.\frac{\partial^{2}}{\partial r^{2}}\left(\lambda_{2} \frac{\partial f_{2}}{\partial \omega_{r r}}\right)\right] \delta \omega d z d r d t+\int_{0}^{t_{f}} \int_{0}^{R} \lambda_{2} \frac{\partial f_{2}}{\partial \omega_{z}} \delta \omega_{\text {int }} d r d t-\int_{0}^{t_{f}} \int_{0}^{R}\left[\lambda_{2} \frac{\partial f_{2}}{\partial \omega_{z}}-\lambda_{3} \frac{\partial f_{3}}{\partial \omega}\right] \delta \omega_{\text {int }} d r d t+$

$\int_{0}^{t_{f}} \int_{0}^{Z}\left[\lambda_{2} \frac{\partial f_{2}}{\partial \omega_{r}}+\frac{\partial \lambda_{2}}{\partial r}\left(\frac{\partial f_{2}}{\delta \omega_{r r}}\right)\right] \delta \omega_{\text {int }} d z d t+\int_{0}^{t_{f}} \int_{0}^{R} \int_{0}^{Z}\left[+\frac{\delta \lambda_{1}}{\delta t}+\lambda_{1} \frac{\partial f_{1}}{\partial T}-\frac{\partial}{\partial z}\left(\lambda_{1} \frac{\partial f_{1}}{\partial T_{z}}\right)+\right.$

$\left.\frac{\partial^{2}}{\partial z^{2}}\left(\lambda_{1} \frac{\partial f_{1}}{\partial T_{z z}}\right)+\frac{\partial^{2}}{\partial r^{2}}\left(\lambda_{1} \frac{\partial f_{1}}{\partial T_{r r}}\right)-\frac{\partial}{\partial r}\left(\lambda_{1} \frac{\partial f_{1}}{\partial T_{r}}\right)\right] \delta T d z d r d t+\int_{0}^{t_{f}} \int_{0}^{R}\left[\lambda_{1} \frac{\partial f_{1}}{\partial T_{z}}+\lambda_{1} \frac{\partial f_{1}}{\partial T_{z z}}-\right.$

$\left.\frac{\partial}{\partial z}\left(\lambda_{1} \frac{\partial f_{1}}{\partial T_{z z}}\right)\right] \delta T_{\text {int }} d r d t-\int_{0}^{t_{f}} \int_{0}^{R}\left[\lambda_{1} \frac{\partial f_{1}}{\partial T_{z}}+\lambda_{1} \frac{\partial f_{1}}{\partial T_{z z}}-\frac{\partial}{\partial z}\left(\lambda_{1} \frac{\partial f_{1}}{\partial T_{z z}}\right)\right] \delta T_{\text {int }} d r d t+\int_{0}^{t_{f}} \int_{0}^{Z}\left[\lambda_{1} \frac{\partial f_{1}}{\partial T_{r}}+\right.$ 
$\left.\frac{\partial \lambda_{1}}{\partial r}\left(\frac{\partial f_{1}}{\partial T_{r r}}\right)\right] \delta T_{\text {int }} d z d t+\int_{0}^{t_{f}} \int_{0}^{R} \int_{0}^{Z} G_{1} \delta \lambda_{1} d z d r d t+$

$\int_{0}^{t_{f}} \int_{0}^{R} \int_{0}^{Z} G_{2} \delta \lambda_{2} d z d r d t+\int_{0}^{t_{f}} \int_{0}^{R} \int_{0}^{Z} H \delta \lambda_{3} d z d r d t=0$

By rearranging Equation (84), it leads to:

$$
\begin{aligned}
& \delta J=\underbrace{\left(\int_{0}^{t_{f}} \int_{0}^{R}\left[-\beta r+\frac{\partial \lambda_{3}}{\partial t}\right] \delta Z d r d t\right)}_{\delta J 1}+ \\
& \underbrace{\left(\int_{0}^{t_{f}} \int_{0}^{R} \int_{0}^{Z}\left[\frac{\partial \lambda_{2}}{\partial t}+\lambda_{2} \frac{\partial f_{2}}{\partial \omega}-\frac{\partial}{\partial z}\left(\lambda_{2} \frac{\partial f_{2}}{\partial \omega_{z}}\right)-\frac{\partial}{\partial r}\left(\lambda_{2} \frac{\partial f_{2}}{\partial \omega_{r}}\right)+\frac{\partial^{2}}{\partial r^{2}}\left(\lambda_{2} \frac{\partial f_{2}}{\partial \omega_{r r}}\right)\right] \delta \omega d z d r d t\right)}_{\delta J 2}+ \\
& \underbrace{\left(\begin{array}{c}
\int_{0}^{t_{f}} \int_{0}^{R} \lambda_{2} \frac{\partial f_{2}}{\partial \omega_{z}} \delta \omega_{\text {int }} d r d t-\int_{0}^{t_{f}} \int_{0}^{R}\left[\lambda_{2} \frac{\partial f_{2}}{\partial \omega_{z}}-\lambda_{3} \frac{\partial f_{3}}{\partial \omega}\right] \delta \omega_{\text {int }} d r d t \\
+\int_{0}^{t_{f}} \int_{0}^{Z}\left[\lambda_{2} \frac{\partial f_{2}}{\partial \omega_{r}}+\frac{\partial \lambda_{2}}{\partial r}\left(\frac{\partial f_{2}}{\delta \omega_{r r}}\right)\right] \delta \omega_{\text {int }} d z d t
\end{array}\right)}_{\delta J 3}+ \\
& \underbrace{\left(\begin{array}{c}
\int_{0}^{t_{f}} \int_{0}^{R} \int_{0}^{Z}+\frac{\delta \lambda_{1}}{\delta t}+\lambda_{1} \frac{\partial f_{1}}{\partial T}-\frac{\partial}{\partial z}\left(\lambda_{1} \frac{\partial f_{1}}{\partial T_{z}}\right)+\frac{\partial^{2}}{\partial z^{2}}\left(\lambda_{1} \frac{\partial f_{1}}{\partial T_{z Z}}\right) \\
+\frac{\partial^{2}}{\partial r^{2}}\left(\lambda_{1} \frac{\partial f_{1}}{\partial T_{r r}}\right)-\frac{\partial}{\partial r}\left(\lambda_{1} \frac{\partial f_{1}}{\partial T_{r}}\right) \delta T d z d r d t
\end{array}\right)+}_{\delta J 4}+ \\
& \underbrace{\left(\begin{array}{c}
\int_{0}^{t_{f}} \int_{0}^{R}\left[\lambda_{1} \frac{\partial f_{1}}{\partial T_{z}}+\lambda_{1} \frac{\partial f_{1}}{\partial T_{z z}}-\frac{\partial}{\partial z}\left(\lambda_{1} \frac{\partial f_{1}}{\partial T_{z z}}\right)\right] \delta T_{\text {int }} d r d t-\int_{0}^{t_{f}} \int_{0}^{R}\left[\lambda_{1} \frac{\partial f_{1}}{\partial T_{z}}+\lambda_{1} \frac{\partial f_{1}}{\partial T_{z z}}-\frac{\partial}{\partial z}\left(\lambda_{1} \frac{\partial f_{1}}{\partial T_{z z}}\right)\right] \delta T_{\text {int }} d r d t+ \\
\int_{0}^{t_{f}} \int_{0}^{Z}\left[\lambda_{1} \frac{\partial f_{1}}{\partial T_{r}}+\frac{\partial \lambda_{1}}{\partial r}\left(\frac{\partial f_{1}}{\partial T_{r r}}\right)\right] \delta T_{i n t} d z d t
\end{array}\right)+}_{\delta J 5} \\
& \underbrace{\left(\int_{0}^{t_{f}} \int_{0}^{R} \int_{0}^{Z} G_{1} \delta \lambda_{1} d z d r d t\right)}_{\delta J 6}+\underbrace{\left(\int_{0}^{t_{f}} \int_{0}^{R} \int_{0}^{Z} G_{2} \delta \lambda_{2} d z d r d t\right)}_{\delta J 7}+\underbrace{\left(\int_{0}^{t_{f}} \int_{0}^{R} \int_{0}^{Z} H \delta \lambda_{3} d z d r d t\right)}_{\delta J 8}=0
\end{aligned}
$$

Assuming that the involved function is sufficiently differentiable, $\delta J$ is zero if all the individual in Equation (85) is zero.

The elimination of the first, second, and fourth terms by defining the variational derivative with respect to $T_{\text {int }}$ and $\omega_{\text {int }}$ :

$$
\frac{\partial \lambda_{3}}{\partial t}=\beta r=2 \pi \rho \emptyset r
$$

$$
0 \leq t \leq t_{f} \text { and } 0 \leq r \leq R
$$

Next, we obtain: 
$\frac{\partial \lambda_{2}}{\partial t}=-\lambda_{2} \frac{\partial f_{2}}{\partial \omega}+\frac{\partial}{\partial z}\left(\lambda_{2} \frac{\partial f_{2}}{\partial \omega_{z}}\right)+\frac{\partial}{\partial r}\left(\lambda_{2} \frac{\partial f_{2}}{\partial \omega_{r r}}\right)-\frac{\partial^{2}}{\partial r^{2}}\left(\lambda_{2} \frac{\partial f_{2}}{\partial \omega_{r r}}\right)$

where

$f_{2}=\left(\frac{\partial D}{\partial \omega}\right) \cdot\left(\frac{\partial \omega}{\partial r}\right)^{2}+\frac{D}{r}\left(\frac{\partial \omega}{\partial r}\right)+D\left(\frac{\partial^{2} \omega}{\partial r^{2}}\right)-3 \alpha \frac{\omega^{2}}{\phi}\left(\frac{\partial \omega}{\partial z}\right)$

Thus, Equation (87) can be written as:

$\frac{\partial \lambda_{2}}{\partial t}=-\frac{3 \alpha}{\emptyset} \omega^{2} \frac{\partial \lambda_{2}}{\partial z}+\frac{\partial \lambda_{2}}{\partial r} \frac{D}{r}-\lambda_{2} \frac{D}{r^{2}}-\frac{\partial^{2} \lambda_{2}}{\partial r^{2}}$

$$
0 \leq t \leq t_{f}, 0 \leq r \leq R,, 0 \leq z \leq Z
$$

Lastly, we have:

$\frac{\partial \lambda_{1}}{\partial t}=-\lambda_{1} \frac{\partial f_{1}}{\partial T}+\frac{\partial}{\partial z}\left(\lambda_{1} \frac{\partial f_{1}}{\partial T_{z}}\right)-\frac{\partial^{2}}{\partial z^{2}}\left(\lambda_{1} \frac{\partial f_{1}}{\partial T_{z z}}\right)-\frac{\partial^{2}}{\partial r^{2}}\left(\lambda_{1} \frac{\partial f_{1}}{\partial T_{r r}}\right)+\frac{\partial}{\partial r}\left(\lambda_{1} \frac{\partial f_{1}}{\partial T_{r}}\right)$

where

$f_{1}=\gamma\left[\frac{1}{r} \frac{\partial T}{\partial r}+\frac{\partial^{2} T}{\partial r^{2}}\right]+\gamma\left[\frac{\partial^{2} T}{\partial z^{2}}\right]-\alpha \frac{\omega^{2}}{\emptyset}\left[\frac{\partial T}{\partial z}\right]$

As a result,

$\frac{\partial \lambda_{1}}{\partial t}=\frac{\partial \lambda_{1}}{\partial z}\left[-\alpha \frac{\omega^{2}}{\varnothing}\right]-\frac{\partial^{2} \lambda_{1}}{\partial z^{2}}[\gamma]-\frac{\partial^{2} \lambda_{1}}{\partial r^{2}}[\gamma]+\frac{\partial \lambda_{1}}{\partial r}\left[\frac{\gamma}{r}\right]$

$$
0 \leq t \leq t_{f}, 0 \leq r \leq R,, 0 \leq z \leq Z
$$

We also need to eliminate the rest of terms in Equation (85), i.e.,

$\left.\int_{0}^{R}\left[\lambda_{2} \frac{\partial f_{2}}{\partial \omega_{z}}\right]_{Z}-\left[\lambda_{2} \frac{\partial f_{2}}{\partial \omega_{z}}-\lambda_{3} \frac{\partial f_{3}}{\partial \omega}\right]_{z=0}\right] d r+\int_{0}^{Z}\left[\lambda_{2} \frac{\partial f_{2}}{\partial \omega_{r}}-\frac{\partial \lambda_{2}}{\partial r} \frac{\partial f_{3}}{\partial \omega_{r r}}\right]_{r=R} d z=\frac{\partial J}{\partial \omega_{\text {int }}}$ 
The above term needs to be zero to make $\partial J$ zero. The above equation is the stationarity condition, and the term is called the variation derivative of $J$ with respect to $\omega_{\text {int }}$.

Equation (91) can be simplified by using Equations (43) and (45)

$$
\begin{aligned}
& \left.\frac{\partial J}{\partial \omega_{\text {int }}}=\int_{0}^{R}\left[-3 \alpha \lambda_{2} \frac{\omega^{2}}{\emptyset}\right]_{z=Z}-\left[-3 \alpha \lambda_{2} \frac{\omega^{2}}{\emptyset}+\frac{2 \lambda_{3} \alpha \omega}{\emptyset}\right]_{Z=0}\right] d r+\int_{0}^{Z}\left[\lambda_{2} \frac{\partial D}{\partial \omega} \frac{\partial \omega}{\partial r}+\lambda_{2} \frac{D}{r}-\right. \\
& \left.\frac{\partial \lambda_{2}}{\partial r} D\right]_{r=R} d z=0
\end{aligned}
$$

$$
0 \leq t \leq t_{f}
$$

similarly:

$\frac{\partial J}{\partial T_{i n t}}=\int_{0}^{R}\left[\lambda_{1} \frac{\partial f_{1}}{\partial T_{z}}+\lambda_{1} \frac{\partial f_{1}}{\partial T_{z z}}-\frac{\partial}{\partial z}\left(\lambda_{1} \frac{\partial f_{1}}{\partial T_{z z}}\right)\right]_{z=Z}-\left[\lambda_{1} \frac{\partial f_{1}}{\partial T_{z}}+\lambda_{1} \frac{\partial f_{1}}{\partial T_{z z}}-\frac{\partial}{\partial z}\left(\lambda_{1} \frac{\partial f_{1}}{\partial T_{z z}}\right)\right]_{z=0} d r+$

$\int_{0}^{Z}\left[\lambda_{1} \frac{\partial f_{1}}{\partial T_{r}}+\frac{\partial \lambda_{1}}{\partial r}\left(\frac{\partial f_{1}}{\partial T_{r r}}\right)\right]_{r=R} d z$

Equation (93) can be simplified by using Equation (41)

$$
\begin{gathered}
\int_{0}^{R}\left[\lambda_{1}\left(-\alpha \frac{\omega^{2}}{\varnothing}\right)+\lambda_{1}(\gamma)-\frac{\partial \lambda_{1}}{\partial z}(\gamma)\right]_{z=Z}-\left[\lambda_{1}\left(-\alpha \frac{\omega^{2}}{\varnothing}\right)+\lambda_{1}(\gamma)-\frac{\partial \lambda_{1}}{\partial z}(\gamma)\right]_{z=0} d r+\int_{0}^{Z}\left[\lambda_{1}\left(\frac{\gamma}{r}\right)+\right. \\
\left.\frac{\partial \lambda_{1}}{\partial r}(\gamma)\right]_{r=R} d z=\frac{\partial J}{\partial T_{\text {int }}} \\
0 \leq t \leq t_{f}
\end{gathered}
$$

Parallel to Equation (91), Equation (94) needs to be zero to help make $\partial J$ zero. The equation above is the stationarity condition, and the term is called the variation derivative of $J$ with respect to $T_{\text {int }}$.

Therefore, $\frac{\partial J}{\partial \omega_{\text {int }}}$ is the variational derivative $\mathrm{J}$ with respect to $\omega_{\text {int }}$ resulting to:

$G_{2}=0$ and $H=0$ 
Similarly, $\frac{\partial J}{\partial T_{\mathrm{int}}(t)}$ is the variational derivative $\mathrm{J}$ with respect to $T_{\mathrm{int}}(t)$ resulting to:

$G_{1}=0$

Lastly, according to Equation (83), we can combine the Equations (92) and (94). Therefore:

$$
\begin{aligned}
& \frac{\partial J}{\partial T_{\text {int }}}=\left[\int_{0}^{R}\left[\lambda_{1}\left(-\alpha \frac{\omega^{2}}{\emptyset}\right)+\lambda_{1}(\gamma)-\frac{\partial \lambda_{1}}{\partial z}(\gamma)\right]_{Z=Z}-\left[\lambda_{1}\left(-\alpha \frac{\omega^{2}}{\emptyset}\right)+\lambda_{1}(\gamma)-\frac{\partial \lambda_{1}}{\partial z}(\gamma)\right]_{Z=0} d r+\right. \\
& \int_{0}^{Z}\left[\lambda_{1}\left(\frac{\gamma}{r}\right)+\frac{\partial \lambda_{1}}{\partial r}(\gamma)\right]_{r=R} d z+\int_{0}^{R}\left[\left[-3 \alpha \lambda_{2} \frac{\omega^{2}}{\emptyset}\right]_{z=Z}-\left[-3 \alpha \lambda_{2} \frac{\omega^{2}}{\emptyset}+2 \alpha \lambda_{3} \frac{\omega}{\emptyset}\right]_{z=0}\right] d r+ \\
& \left.\int_{0}^{Z}\left[\lambda_{2} \frac{\partial D}{\partial \omega} \frac{\partial \omega}{\partial r}+\lambda_{2} \frac{D}{r}-\frac{\partial \lambda_{2}}{\partial r} D\right]_{r=R} d z\right]=0
\end{aligned}
$$

For the $J$ function to be optimal, the necessary conditions given by Equation (95) must be satisfied along with Equations (26), (30), (34), and the costate equations. If the $J$ function is not optimal, then a correction in the gradient direction ensures an improvement in the objective function. It worth to mention that discretization of $\left[T_{\text {int }}(t)\right]$, and cubic splines were used to obtain $\left[T_{\text {int }}(t)\right]$ when solving the mathematical modeling.

\subsection{Implementation of the Improvement Method}

The search direction was set by the variation derivative $J_{T \text { int }}$ given by:

$$
\begin{aligned}
& \frac{\partial J}{\partial T_{\text {int }}}=J_{T_{\text {int }}}=\left[\int_{0}^{R}\left[\lambda_{1}\left(-\alpha \frac{\omega^{2}}{\emptyset}\right)+\lambda_{1}(\gamma)-\frac{\partial \lambda_{1}}{\partial z}(\gamma)\right]_{z=Z}-\left[\lambda_{1}\left(-\alpha \frac{\omega^{2}}{\emptyset}\right)+\lambda_{1}(\gamma)-\right.\right. \\
& \left.\frac{\partial \lambda_{1}}{\partial z}(\gamma)\right]_{z=0} d r+\int_{0}^{Z}\left[\lambda_{1}\left(\frac{\gamma}{r}\right)+\frac{\partial \lambda_{1}}{\partial r}(\gamma)\right]_{r=R} d z+\int_{0}^{R}\left[\left[-3 \alpha \lambda_{2} \frac{\omega^{2}}{\emptyset}\right]_{z=Z}-\left[-3 \alpha \lambda_{2} \frac{\omega^{2}}{\emptyset}+\right.\right. \\
& \left.\left.\left.\frac{2 \lambda_{3} \alpha \omega}{\emptyset}\right]_{z=0}\right] d r+\int_{0}^{Z}\left[\lambda_{2} \frac{\partial D}{\partial \omega} \frac{\partial \omega}{\partial r}+\lambda_{2} \frac{D}{r}-\frac{\partial \lambda_{2}}{\partial r} D\right]_{r=R} d z\right]=0
\end{aligned}
$$


The gradient correction $J_{T_{\text {int }}}$ was expressed in a finite-difference form along $r$ and $z$ directions to be utilized in the simulation. The values of differential changes are time-averaged before their usage for the gradient correction in $\left(T_{\mathrm{int}}\right)$ by the Broyden-Fletcher-Goldfarb-Shanno (BFGS) method. The iterative improvement in the value of $\left(T_{\mathrm{int}}\right)$ is given by:

$T_{\mathrm{int}, i+1}=T_{\mathrm{int}, i}-\beta_{i} \cdot J_{T_{\mathrm{int}}}$

where $\beta_{i}$ is the optimal step length along the search direction in the $i^{t h}$ iteration determined by the BFGS algorithm.

\subsubsection{The Gradient Improvement Method}

A gradient method was adopted for the iterative solution of this optimal control problem. In more detail, the maximum local rate of increase in the value of a function with respect to its variables is in the direction to its gradient. This progress is called the steepest ascent direction. Equation (48) is used to set the search path to find the maximum of $J .^{48,49}$

Quasi-Newton methods are the most favored of the methods that use a gradient of a function. These methods compute a search direction utilizing first order gradient information. Newton's methods make use of the second order information (Hessian matrix) of a function. Hessian matrix is calculated numerically, which involves a significant amount of computations. ${ }^{48,49}$

Quasi-Newton methods avoid the calculation of exact Hessian by generating approximate Hessian matrix using an appropriate updating technique. Among different Quasi-Newton methods, the BFGS method is consider as one of the most efficient method. ${ }^{48,49}$ In more detail, BFGS method 
is a computational algorithm to find an optimal solution for unconstrained nonlinear problems. It utilizes first order gradient information to generate the approximate Hessian matrix. ${ }^{48}$

\subsection{The Optimal Control Algorithm}

Figure 6 shows the schematic of the computational algorithm that was utilized to determine $T_{\text {int }}$ versus time. Programmed in $\mathrm{C}++$ language, the developed algorithm was implemented on Itanium 2/ Intel Itanium processor (64 bit, $1.5 \mathrm{GHz}, 15.9 \mathrm{~GB}$ of RAM) with Intel C++ compiler.

Lastly, we reduced the computation time from 2 weeks to 5 days by scaling the dependent variables of the differential equations. The following are the steps of the computational algorithm, which improves $\left[T_{\text {int }}(t)\right]$ in order to maximize $J$ or $I$, i.e., oil production:

(i) Provide the initial guess for interface temperature function $\left[T_{\mathrm{int}}(t)\right]$ as vector of discrete values is the time domain, $0-\mathrm{t}_{\mathrm{f}}$.

a. Initial guess of $T_{\mathrm{int}}$ is very important, and it needs a considerable attention (Please see Section 5.2.3)

(ii) Integrate the mass, heat, and change of height equations, respectively, i.e., Equations (26), (30), and (34) use the initial and control function to obtain the values of state variables $T(t, r, z), \omega(t, r, z)$, and $Z(t, r)$ at each node(t,r,z). Remember to save the values of state variables at the discretized points.

(iii) Evaluate the objective functional use the values of control functions, and state variables. Again save the objective functional value. 
(iv) Integrate costate equations backward using final conditions, i.e., Equations (86), (88), and (90) using final condition, the control function, and saved values of state variables. Save the values of costate variables at the discretized point.

(v) Improve of $\left[T_{\text {int }}(\mathrm{t})\right]$ by using the gradient correction $\left[J_{T_{\text {int }}}(\mathrm{t})\right]$ given by Equation (95).

(vi) Repeat computation, and by updating $T_{\text {int }}(\mathrm{t})$, until there is no further improvement in the objective functional value. At this point, the control function has reached its local optimal condition. 
Initial Guess of the Function $T_{\text {int }}(t)$

Integrate forward:

$$
\begin{gathered}
\frac{\partial T}{\partial t}=\gamma\left[\frac{1}{r} \frac{\partial T}{\partial r}+\frac{\partial^{2} T}{\partial r^{2}}\right]+\gamma\left[\frac{\partial^{2} T}{\partial z^{2}}\right]-\alpha \frac{\omega^{2}}{\emptyset}\left[\frac{\partial T}{\partial z}\right] \\
\frac{\partial Z}{\partial t}=\frac{-v_{o}(r)}{\emptyset} \\
\frac{\partial \omega}{\partial t}=\left(\frac{\partial D}{\partial \omega}\right) \cdot\left(\frac{\partial \omega}{\partial r}\right)^{2}+\frac{D}{r}\left(\frac{\partial \omega}{\partial r}\right)+D\left(\frac{\partial^{2} \omega}{\partial r^{2}}\right)-3 \alpha \frac{\omega^{2}}{\phi}\left(\frac{\partial \omega}{\partial z}\right)
\end{gathered}
$$

:

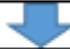

Save

$T(t, r, z), \omega(t, r, z)$ and $Z(t, r)$

\section{Calculate $I$}

Did objective

functional improved!?

\section{Yes}

Integrate backward:

$$
\begin{gathered}
\frac{\partial \lambda_{1}}{\partial t}=\frac{\partial \lambda_{1}}{\partial z}\left[-\alpha \frac{\omega^{2}}{\emptyset}\right]-\frac{\partial^{2} \lambda_{1}}{\partial z^{2}}[\gamma]-\frac{\partial^{2} \lambda_{1}}{\partial r^{2}}[\gamma]+\frac{\partial \lambda_{1}}{\partial r}\left[\frac{\gamma}{r}\right] \frac{\partial \lambda_{2}}{d t} \\
\frac{\partial \lambda_{2}}{\partial t}=-\frac{3 \alpha}{\emptyset} \omega^{2} \frac{\partial \lambda_{2}}{\partial z}+\frac{\partial \lambda_{2}}{\partial r} \frac{D}{r}-\lambda_{2} \frac{D}{r^{2}}-\frac{\partial^{2} \lambda_{2}}{\partial r^{2}} \\
\frac{\partial \lambda_{3}}{\partial t}=\beta r=2 \pi \rho \emptyset r
\end{gathered}
$$

Get $\lambda_{1}(t, r, z), \lambda_{2}(t, r, z)$ and $\lambda_{3}(t, r)$ for each node with respect to time

$$
\text { Improve } T_{\text {int }}(t) \text { using } \frac{\partial J}{\partial T_{\text {int }}(t)}
$$

Figure 6: The Optimal Control Algorithm 


\subsection{Discretized Mathematical Model}

Given that Equations (26), (30), and (34) are partial differential equations (PDEs), there finitedifferenced forms along $r$ and $z$ directions were obtained, which were sets of ordinary differential equations (ODEs). As it can be seen in Figure 5, the cylindrical model is divided into equi-spaced grid points of numbers $\mathrm{N}_{r}$ and $\mathrm{N}_{z}$, respectively, along the $r$ and $z$ directions. An adaptive step size control method is used in the algorithm to achieve the accuracy in the solution with minimum computational effort. ${ }^{48,49}$

\subsubsection{Finite Difference for conversion of PDE to ODE for Heat Transfer}

$\frac{\partial T}{\partial t}=\gamma\left[\frac{1}{r} \frac{\partial T}{\partial r}+\frac{\partial^{2} T}{\partial r^{2}}\right]+\gamma\left[\frac{\partial^{2} T}{\partial z^{2}}\right]-\alpha \frac{\omega^{2}}{\emptyset}\left[\frac{\partial T}{\partial z}\right]$

For Intermediate Grid Points:

for $0<i<\left(N_{i}-1\right)$ and $0<j<\left(N_{j}-1\right)$

$\frac{\partial T_{i, j}}{\partial t}=(\gamma)\left[\frac{1}{r_{i}} \frac{T_{i+1, j}-T_{i-1, j}}{2 \Delta r}+\frac{T_{i+1, j}-2 T_{i, j}+T_{i-1, j}}{\Delta r^{2}}\right]+\gamma\left[\frac{T_{i, j+1}-2 T_{i, j}+T_{i, j-1}}{\Delta z^{2}}\right]-\alpha \frac{\omega^{2}}{\emptyset}\left[\frac{T_{i, j+1}-T_{i, j-1}}{2 \Delta z}\right]$

For Axial Grid Points:

When $i=0$ for $0<j<\left(N_{j}-1\right)$

$\frac{\partial T_{0, j}}{\partial t}=\gamma\left[\frac{T_{0, j+1}-2 T_{0, j}+T_{0, j-1}}{\Delta z^{2}}\right]-\alpha \frac{\omega^{2}}{\emptyset}\left[\frac{T_{0, j+1}-T_{0, j-1}}{2 \Delta z_{i}}\right]$

When $i=0$ and $j=0$;

$\frac{\partial T_{0,0}}{\partial t}=\gamma\left[\frac{T_{0,1}-2 T_{0,0}+T_{i n t}}{\Delta z^{2}}\right]-\alpha \frac{\omega^{2}}{\emptyset}\left[\frac{T_{0,1}-T_{\text {int }}}{2 \Delta z_{0}}\right]$

When $i=0$ and $j=\left(N_{j}-1\right)$

$\frac{\partial T_{0, N \mathrm{j}-1}}{\partial t}=\gamma\left[\frac{T_{\mathrm{int}}-2 T_{0, N j-1}+T_{0, N j-2}}{\Delta z^{2}}\right]-\alpha \frac{\omega^{2}}{\emptyset}\left[\frac{T_{\mathrm{int}}-T_{0, N j-2}}{2 \Delta z_{0}}\right]$ 
For Right Most Grid Points:

When $i=\left(N_{i}-1\right)$ and $j=0$

$\frac{\partial T_{N i-1,0}}{\partial t}=(\gamma)\left[\frac{1}{r_{N i-1}} \frac{T_{i n t}-T_{N i-2,0}}{2 \Delta r}+\frac{T_{(t)}-2 T_{N i-1,0}+T_{N i-2,0}}{\Delta r^{2}}\right]+\delta\left[\frac{T_{N i-1,1}-2 T_{N i-1,0}+T_{i n t}}{\Delta z^{2}}\right]-$ $\alpha \frac{\omega^{2}}{\emptyset}\left[\frac{T_{N i-1,1}-T_{i n t}}{2 \Delta z_{N i-1}}\right]$

When $i=\left(N_{i}-1\right)$

$\frac{\partial T_{N i-1, j}}{\partial t}=(\gamma)\left[\frac{1}{r_{N i-1}} \frac{T_{i n t}-T_{N i-2, j}}{2 \Delta r}+\frac{T_{(t)}-2 T_{N i-1, j}+T_{N i-2, j}}{\Delta r^{2}}\right]+\gamma\left[\frac{T_{N i-1, j+1}-2 T_{N i-1, j}+T_{N i-1, j-1}}{\Delta z^{2}}\right]-$ $\alpha \frac{\omega^{2}}{\emptyset}\left[\frac{T_{N i-1, j+1}-T_{N i-1, j-1}}{2 \Delta z_{N i-1}}\right]$

When $i=\left(N_{i}-1\right) \& j=\left(N_{j}-1\right)$

$\frac{\partial T_{N i-1, N j-1}}{\partial t}=(\gamma)\left[\frac{1}{r_{N i-1}} \frac{T_{i n t}-T_{N i-2, N j-1}}{2 \Delta r}+\frac{T_{i n t}-2 T_{N i-1, N j-1}+T_{N i-2, N j-1}}{\Delta r^{2}}\right]+$

$\gamma\left[\frac{T_{i n t}-2 T_{N i-1, n j-1}+T_{N i-1, N j-2}}{\Delta z^{2}}\right]-\alpha \frac{\omega^{2}}{\emptyset}\left[\frac{T_{i n t}-T_{N i-1, N j-2}}{2 \Delta z_{N i-1}}\right]$

For Lower Most Intermediate Grid Points:

When $0<i<\left(N_{r}-1\right)$ and $j=0$

$\frac{\partial T_{i, 0}}{\partial t}=(\gamma)\left[\frac{1}{r_{i}} \frac{T_{i+1,0}-T_{i-1,0}}{2 \Delta r}+\frac{T_{i+1,0}-2 T_{i, 0}+T_{i-1,0}}{\Delta r^{2}}\right]+\gamma\left[\frac{T_{i, 1}-2 T_{i, 0}+T_{i n t}}{\Delta z^{2}}\right]-\alpha \frac{\omega^{2}}{\emptyset}\left[\frac{T_{i, 1}-T_{i n t}}{2 \Delta z_{i}}\right]$

For Upper Most Intermediate Grid Points:

When $0<i<\left(N_{r}-1\right)$ and $j=N_{z}-1$

$\frac{\partial T_{i, N j-1}}{\partial t}=(\gamma)\left[\frac{1}{r_{i}} \frac{T_{i+1, N j-1}-T_{i-1, N j-1}}{2 \Delta r}+\frac{T_{i+1, N j-1}-2 T_{i, N j-1}+T_{i-1, N j-1}}{\Delta r^{2}}\right]+\gamma\left[\frac{T_{i n t}-2 T_{i, N j-1}+T_{i, N j-2}}{\Delta z^{2}}\right]-$

$\alpha \frac{\omega^{2}}{\emptyset}\left[\frac{T_{i n t}-T_{i, N j-2}}{2 \Delta z_{i}}\right]$

where $T_{i, j}$ is the temperature of air at the node, $(i, j)$ corresponding to the coordinate, $r$ and $z$. The distance between grid points are $\Delta r$ and $\Delta z_{i}$ respectively along $r$ and $z$ directions. $\Delta r$ is constant and given by: 


$$
\Delta r=\frac{R}{N_{r}}
$$

But $\Delta z_{i}$ varies with time along $r$ direction, and is given by:

$$
\Delta z_{i}=\frac{z_{i}}{N_{z}+1}
$$

\subsubsection{The Costate Equations for Heat Transfer}

The ordinary differential equations of costate equations for each corresponding grid point can be written as:

For Intermediate Grid Points:

for $0<i<\left(N_{i}-1\right)$ and $0<j<\left(N_{j}-1\right)$

$\frac{\partial \lambda_{i, j}}{\partial t}=(\gamma)\left[\frac{1}{r_{i}} \frac{\lambda_{i+1, j}-\lambda_{i-1, j}}{2 \Delta r}+\frac{\lambda_{i+1, j}-2 \lambda_{i, j}+\lambda_{i-1, j}}{\Delta r^{2}}\right]+\gamma\left[\frac{\lambda_{i, j+1}-2 \lambda_{i, j}+\lambda_{i, j-1}}{\Delta z^{2}}\right]-\alpha \frac{\omega^{2}}{\varnothing}\left[\frac{\lambda_{i, j+1}-\lambda_{i, j-1}}{2 \Delta z}\right]$

For Axial Grid Points:

When $i=0$ for $0<j<\left(N_{j}-1\right)$

$\frac{\partial \lambda_{0, j}}{\partial t}=0$

When $i=0$ and $j=0$;

$\frac{\partial \lambda_{0,0}}{\partial t}=0$

When $i=0$ and $j=\left(N_{j}-1\right)$

$\frac{\partial \lambda_{0, N j-1}}{\partial t}=0$

For Right Most Grid Points:

When $i=\left(N_{i}-1\right)$ and $j=0$ 
$\frac{\partial \lambda_{N i-1,0}}{\partial t}=(\gamma)\left[\frac{1}{r_{N i-1}} \frac{-\lambda_{N i-2,0}}{2 \Delta r}+\frac{-2 \lambda_{N i-1,0}+\lambda_{N i-2,0}}{\Delta r^{2}}\right]+\gamma\left[\frac{\lambda_{N i-1,1}-2 \lambda_{N i-1,0}}{\Delta z^{2}}\right]-\alpha \frac{\omega^{2}}{\emptyset}\left[\frac{\lambda_{N i-1,1}}{2 \Delta z_{N i-1}}\right]$

When $i=\left(N_{i}-1\right)$

$\frac{\partial \lambda_{N i-1, j}}{\partial t}=(\gamma)\left[\frac{1}{r_{N i-1}} \frac{-\lambda_{N i-2, j}}{2 \Delta r}+\frac{-2 \lambda_{N i-1, j}+\lambda_{N i-2, j}}{\Delta r^{2}}\right]+\gamma\left[\frac{\lambda_{N i-1, j+1}-2 \lambda_{N i-1, j}+\lambda_{N i-1, j-1}}{\Delta z^{2}}\right]-$

$\alpha \frac{\omega^{2}}{\emptyset}\left[\frac{\lambda_{N i-1, j+1}-\lambda_{N i-1, j-1}}{2 \Delta z_{N i-1}}\right]$

When $i=\left(N_{i}-1\right)$ and $j=\left(N_{j-1}\right)$

$\frac{\partial \lambda_{n i-1, n j-1}}{\partial t}=(\gamma)\left[\frac{1}{r_{N i-1}} \frac{-\lambda_{N i-2, N j-1}}{2 \Delta r}+\frac{-2 \lambda_{N i-1, N j-1}+\lambda_{N i-2, N j-1}}{\Delta r^{2}}\right]+\gamma\left[\frac{-2 \lambda_{N i-1, N j-1}+\lambda_{N i-1, N j-2}}{\Delta z^{2}}\right]-$

$\alpha \frac{\omega^{2}}{\emptyset}\left[\frac{-\lambda_{N i-1, N j-2}}{2 \Delta z_{N i-1}}\right]$

For Lower Most Intermediate Grid Points:

When $0<i<\left(N_{r}-1\right)$ and $j=0$

$\frac{\partial \lambda_{i, 0}}{\partial t}=(\gamma)\left[\frac{1}{r_{i}} \frac{\lambda_{i+1,0}-\lambda_{i-1,0}}{2 \Delta r}+\frac{\lambda_{i+1,0}-2 \lambda_{i, 0}+\lambda_{i-1,0}}{\Delta r^{2}}\right]+\gamma\left[\frac{\lambda_{i, 1}-2 \lambda_{i, 0}}{\Delta z^{2}}\right]-\alpha \frac{\omega^{2}}{\emptyset}\left[\frac{\lambda_{i, 1}}{2 \Delta z_{i}}\right]$

For Upper Most Intermediate Grid Points:

When $0<i<\left(N_{r}-1\right)$ and $j=N_{z}-1$

$\frac{\partial \lambda_{i, N j-1}}{\partial t}=(\gamma)\left[\frac{1}{r_{i}} \frac{\lambda_{i+1, N j-1}-\lambda_{i-1, N j-1}}{2 \Delta r}+\frac{\lambda_{i+1, N j-1}-2 \lambda_{i, N j-1}+\lambda_{i-1, N j-1}}{\Delta r^{2}}\right]+\gamma\left[\frac{-2 \lambda_{i, N j-1}+\lambda_{i, N j-2}}{\Delta z^{2}}\right]-$

$\alpha \frac{\omega^{2}}{\emptyset}\left[\frac{-\lambda_{i, N j-2}}{2 \Delta z_{i}}\right]$ 


\subsubsection{Finite Difference for conversion of PDE to ODE for Mass Transfer}

$\frac{\partial \omega}{\partial t}=\left(\frac{\partial D}{\partial \omega}\right) \cdot\left(\frac{\partial \omega}{\partial r}\right)^{2}+\frac{D}{r}\left(\frac{\partial \omega}{\partial r}\right)+D\left(\frac{\partial^{2} \omega}{d r^{2}}\right)-3 \alpha \frac{\omega^{2}}{\phi}\left(\frac{\partial \omega}{\partial z}\right)$

For Intermediate Grid Points:

for $0<i<\left(N_{i}-1\right)$ and $0<j<\left(N_{j}-1\right)$

$\frac{\partial \omega_{i, j}}{\partial t}=D\left[\frac{1}{r_{i}} \frac{\omega_{i+1, j}-\omega_{i-1, j}}{2 \Delta r}+\frac{\omega_{i+1, j}-2 \omega_{i, j}+\omega_{i-1, j}}{\Delta r^{2}}\right]+\frac{\partial D}{\partial \omega}\left[\frac{\omega_{i+1, j}-\omega_{i-1, j}}{2 \Delta r}\right]^{2}-\frac{\alpha}{\emptyset} 3 \omega_{i, j}{ }^{2}\left[\frac{\omega_{i, j+1}-\omega_{i, j-1}}{2 \Delta z_{i}}\right]$

For Axial Grid Points:

When $i=0$ and $0<j<\left(N_{j}-1\right)$

$\frac{\partial \omega_{o, j}}{\partial t}=-\frac{\alpha}{\varnothing} 3 \omega_{0, j}^{2}\left[\frac{\omega_{0, j+1}-\omega_{0, j-1}}{2 \Delta z_{i}}\right]$

When $i=0$ and $j=0$

$\frac{\partial \omega_{0,0}}{\partial t}=-\frac{\alpha}{\varnothing} 3 \omega_{0,0}^{2}\left[\frac{\omega_{0,1}-\omega_{\text {int }}}{2 \Delta z_{0}}\right]$

When $i=0$ and $j=\left(N_{j-}-1\right)$

$\frac{\partial \omega_{0, N j-1}}{\partial t}=-\frac{\alpha}{\emptyset} 3 \omega_{0, N j-1}^{2}\left[\frac{\omega_{i n t}-\omega_{0, N j-2}}{2 \Delta z_{0}}\right]$

For Right Most Grid Points:

When $i=\left(N_{i}-1\right)$ and $j=0$

$\frac{\partial \omega_{N i-1,0}}{\partial t}=D\left[\frac{1}{r_{N i-1}} \frac{\omega_{i n t}-\omega_{N i-2,0}}{2 \Delta r}+\frac{\omega_{i n t}-2 \omega_{N i-1,0}+\omega_{N i-2,0}}{\Delta r^{2}}\right]+\frac{\partial D}{\partial \omega}\left[\frac{\omega_{i n t}-\omega_{N i-2,0}}{2 \Delta r}\right]^{2}-$ $\frac{\alpha}{\emptyset} 3 \omega_{N i-1,0}^{2}\left[\frac{\omega_{N i-1,1}-\omega_{i n t}}{2 \Delta z_{N i-1}}\right]$

When $i=\left(N_{i}-1\right)$ 
$\frac{\partial \omega_{N i-1, j}}{\partial t}=D\left[\frac{1}{r_{N i-1}} \frac{\omega_{i n t}-\omega_{N i-2, j}}{2 \Delta r}+\frac{\omega_{i n t}-2 \omega_{N i-1, j}+\omega_{N i-2, j}}{\Delta r^{2}}\right]+\frac{\partial D}{\partial \omega}\left[\frac{\omega_{i n t}-\omega_{N i-2, j}}{2 \Delta r}\right]^{2}-$

$\frac{\alpha}{\varnothing} 3 \omega_{N i-1, j}^{2}\left[\frac{\omega_{N i-1, j+1}-\omega_{n i-1, j-1}}{2 \Delta z_{N i-1}}\right]$

When $i=\left(N_{i}-1\right)$ and $j=\left(N_{j}-1\right)$

$\frac{\partial \omega_{N i-1, N j-1}}{\partial t}=D\left[\frac{1}{r_{n i-1}} \frac{\omega_{i n t}-\omega_{N i-2, N j-1}}{2 \Delta r}+\frac{\omega_{i n t}-2 \omega_{N i-1, N j-1}+\omega_{N i-2, N j-1}}{\Delta r^{2}}\right]+\frac{\partial D}{\partial \omega}\left[\frac{\omega_{i n t}-\omega_{N i-2, N j-1}}{2 \Delta r}\right]^{2}-$

$\frac{\alpha}{\emptyset} 3 \omega_{N i-1, N j-1}^{2}\left[\frac{\omega_{i n t}-\omega_{N i-1, N j-2}}{2 \Delta z_{N i-1}}\right]$

For Lower Most Intermediate Grid Points:

When $0<i<\left(N_{r}-1\right)$ and $j=0$

$\frac{\partial \omega_{i, 0}}{\partial t}=D\left[\frac{1}{r_{i}} \frac{\omega_{i+1,0}-\omega_{i-1,0}}{2 \Delta r}+\frac{\omega_{i+1,0}-2 \omega_{i, 0}+\omega_{i-1,0}}{\Delta r^{2}}\right]+\frac{\partial D}{\partial \omega}\left[\frac{\omega_{i+1,0}-\omega_{i-1,0}}{2 \Delta r}\right]^{2}-\frac{\alpha}{\emptyset} 3 \omega_{i, 0}^{2}\left[\frac{\omega_{i, 1}-\omega_{i n t}}{2 \Delta z_{i}}\right]$

For Upper Most Intermediate Grid Points:

When $0<i<\left(N_{r}-1\right)$ and $j=N_{z^{-}}-1$

$\frac{\partial \omega_{i, N j-1}}{\partial t}=D\left[\frac{1}{r_{i}} \frac{\omega_{i+1, N j-1}-\omega_{i-1, N j-1}}{2 \Delta r}+\frac{\omega_{i+1, N j-1}-2 \omega_{i, N j-1}+\omega_{i-1, N j-1}}{\Delta r^{2}}\right]+\frac{\partial D}{\partial \omega}\left[\frac{\omega_{i+1, N j-1}-\omega_{i-1, N j-1}}{2 \Delta r}\right]^{2}-$

$\frac{\alpha}{\emptyset} 3 \omega_{i, N j-1}^{2}\left[\frac{\omega_{i n t}-\omega_{i, N j-2}}{2 \Delta z_{i}}\right]$

where $\omega_{i, j}$ is the mass fraction of atmospheric air at the node, $(i, j)$ corresponding to the coordinate, $r$ and $z$. 


\subsubsection{The Costate Mass Transfer Equations}

The ordinary differential equations of costate equations written for the corresponding grid points are as follows:

For Intermediate Grid points:

for $0<i<\left(N_{i}-1\right)$ and $0<j<\left(N_{j}-1\right)$

$\frac{\partial \lambda_{i, j}}{\partial t}=D\left[\frac{1}{r_{i}} \frac{\lambda_{i+1, j}-\lambda_{i-1, j}}{2 \Delta r}-\frac{\lambda_{i+1, j}-2 \lambda_{i, j}+\lambda_{i-1, j}}{\Delta r^{2}}\right]+D\left[\frac{\lambda_{i, j}}{r_{i}^{2}}\right]-\frac{\alpha}{\varnothing} 3 \omega_{i, j}{ }^{2}\left[\frac{\lambda_{i, j+1}-\lambda_{i, j-1}}{2 \Delta z_{i}}\right]$

For Axial Grid Points:

When $i=0$ and $0<j<\left(N_{j}-1\right)$

$\frac{\partial \lambda_{0,0}}{\partial t}=\frac{\partial \lambda_{0, j}}{\partial t}=\frac{\partial \lambda_{0, N Z-1}}{\partial t}=0$

For the Right Most Grid Points:

When $i=\left(N_{i}-1\right)$ and $j=0$

$\frac{\partial \lambda_{N i-1,0}}{\partial t}=D\left[\frac{1}{r_{N i-1}} \frac{-\lambda_{N i-2,0}}{2 \Delta r}-\frac{-2 \lambda_{N i-1,0}+\lambda_{N i-2,0}}{\Delta r^{2}}\right]-D\left[\frac{\lambda_{N i-1,0}}{r_{N i-1}^{2}}\right]-\frac{\alpha}{\varnothing} 3 \lambda_{N i-1,0}{ }^{2}\left[\frac{\lambda_{N i-1,1}}{2 \Delta z_{N i-1}}\right]$

When $i=\left(N_{i}-1\right)$ and $0<j<\left(N_{z}-1\right)$

$\frac{\partial \lambda_{N i-1, j}}{\partial t}=D\left[\frac{1}{r_{N i-1}} \frac{-\lambda_{N i-2, j}}{2 \Delta r}-\frac{-2 \lambda_{N i-1, j}+\lambda_{N i-2, j}}{\Delta r^{2}}\right]-D\left[\frac{\lambda_{N i-1,0}}{r_{N i-1}^{2}}\right]-\frac{\alpha}{\phi} 3 \omega_{N i-1, j}{ }^{2}\left[\frac{\lambda_{N i-1, j}-\lambda_{N i-1, j-1}}{2 \Delta z_{N i-1}}\right]$

When $i=\left(N_{i}-1\right)$ and $j=\left(N_{j}-1\right)$

$\frac{\partial \lambda_{N i-1, N Z-1}}{\partial t}=D\left[\frac{1}{r_{N i-1}} \frac{-\lambda_{N i-2, N j-1}}{2 \Delta r}-\frac{-2 \lambda_{N i-1, N j-1}+\omega_{N i-2, N j-1}}{\Delta r^{2}}\right]-D\left[\frac{\lambda_{N i-1, N j-1}}{r_{N i-1}^{2}}\right]-$

$\frac{\alpha}{\varnothing} 3 \lambda_{N i-1, N j-1}{ }^{2}\left[\frac{-\lambda_{N i-1, N j-2}}{2 \Delta z_{N i-1}}\right]$

For Lower Most Intermediate Grid Points:

When $0<i<\left(N_{r}-1\right)$ and $j=0$ 
$\frac{\partial \lambda_{i, 0}}{\partial t}=D\left[\frac{1}{r_{i}} \frac{\lambda_{i+1,0}-\lambda_{i-1,0}}{2 \Delta r}+\frac{\lambda_{i+1,0}-2 \lambda_{i, 0}+\lambda_{i-1,0}}{\Delta r^{2}}\right]-D\left[\frac{\lambda_{i, 0}}{r_{i}^{2}}\right]-\frac{\alpha}{\emptyset} 3 \lambda_{i, 0}^{2}\left[\frac{\lambda_{i, 1}}{2 \Delta z_{i}}\right]$

For Upper Most Intermediate Grid Points:

When $0<i<\left(N_{r^{-}}-1\right)$ and $j=N_{z^{-}}-1$

$\frac{\partial \lambda_{i, N j-1}}{\partial t}=D\left[\frac{1}{r_{i}} \frac{\lambda_{i+1, N j-1}-\lambda_{i-1, N j-1}}{2 \Delta r}+\frac{\lambda_{i+1, N j-1}-2 \lambda_{i, N j-1}+\lambda_{i-1, N j-1}}{\Delta r^{2}}\right]-D\left[\frac{\lambda_{i, N j-1}}{r_{i}^{2}}\right]-$

$\frac{\alpha}{\emptyset} 3 \lambda_{i, N j-1}^{2}\left[\frac{-\lambda_{i, N j-2}}{2 \Delta z_{i}}\right]$

\subsubsection{Change in Height of Physical Model}

Change in the height of the physical model at any time instance was calculated by averaging the mass fraction value for the exposed nodes to solve gas at the bottom of the model.

The second set of ordinary differential equations were written as:

The change in height for $0 \leq i<\left(N_{r}-1\right)$ is given by:

$\frac{\partial z_{i}}{\partial t}=\frac{-v_{i, 0}}{\emptyset}=-3 \frac{\alpha}{\emptyset}\left[\frac{\omega_{i, 0}-\omega_{i+1,0}+2 \omega_{\mathrm{int}}}{4}\right]^{2}$

For $i=N_{r}-1$

$\frac{\partial Z_{N i-1}}{\partial t}=\frac{-v_{N i-1,0}}{\varnothing}=-3 \frac{\alpha}{\varnothing}\left[\frac{\omega_{N i-1,0}+3 \omega_{i n t}}{4}\right]^{2}$ 


\section{Chapter 5: Results and Discussion}

This chapter presents the results obtained from the experiments carried out using the experimental setups. We examine the impact of permeability, pressure, and temperature of atmospheric air injection on heavy oil recovery. Next, the results obtained from the application of optimal control are assessed, and the optimal control policy is experimentally validated.

\subsection{Preliminary Results}

The sections below present the results of different experiments carried out to recover heavy oil from the physical reservoir models using atmospheric air injection. First, we did experiments with four different reservoir model permeabilities of 40, 87, 204 and 427 Darcy. Next, we performed experiments with four different pressures of $0.169,0.286,0.403$ and $0.514 \mathrm{MPa}$ absolute at $25^{\circ} \mathrm{C}$ and with 204 Darcy model permeability. Lastly, we did experiments with various air temperatures in the range $25-90^{\circ} \mathrm{C}$ with (i) constant air temperature, and (ii) periodic air temperature variations with time. The maximum temperature of the air was $90^{\circ} \mathrm{C}$ in the experiments. No chemical reaction took place in the experiments according to the analysis of effluent air, reservoir model temperature, and live oil viscosity (please see Section 5.1.5). We have also carried experiments using injection of pure nitrogen gas (please see Appendix C-F)

\subsubsection{Effect of Permeability on Heavy Oil Recovery}

To determine the effect of permeability on oil recovery, experiments were carried out at air temperature and pressure of $25^{\circ} \mathrm{C}$ and $0.514 \mathrm{MPa}$, respectively, and different permeability values of 40, 87, 204, and 427 Darcy. The results are shown in Figure 7. It is observed that the recovery increases with permeability. This result is in close agreement with what is reported in the 


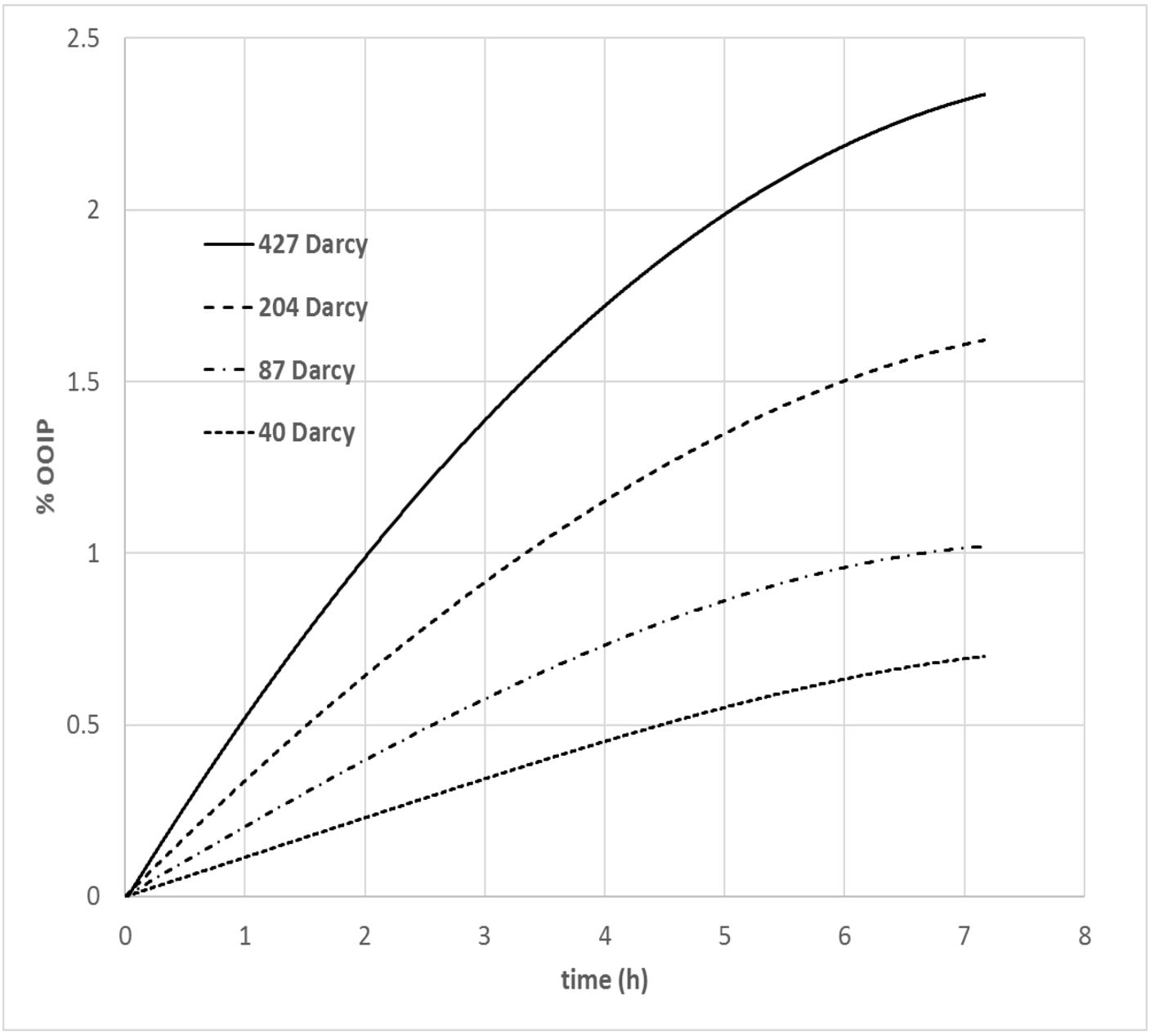

Figure 7: Oil recovery of air injection versus time for different permeabilities at $0.514 \mathrm{MPa}$ absolute and $25^{\circ} \mathrm{C}$.

literature $^{52,53}$ and is attributed to the fact that the diluted oil can drain due to gravity more easily through the physical model of higher permeability. ${ }^{26}$ The overall oil recovery in these experiments was found to increase from 0.70 to $2.33 \%$ of the OOIP as the permeability increased from 40 to 427 Darcy. Furthermore, the correlation between the production rate and permeability is shown in Figure 8 with the $r^{2}$ value of 0.9995 . The production rate $\left[\dot{m} \times 10^{3}(\mathrm{gram} / \mathrm{min})\right]$ and the physical model permeability $K$ (Darcy) is given by the following equation:

$\dot{m}=0.5251 K^{0.5133}$ 


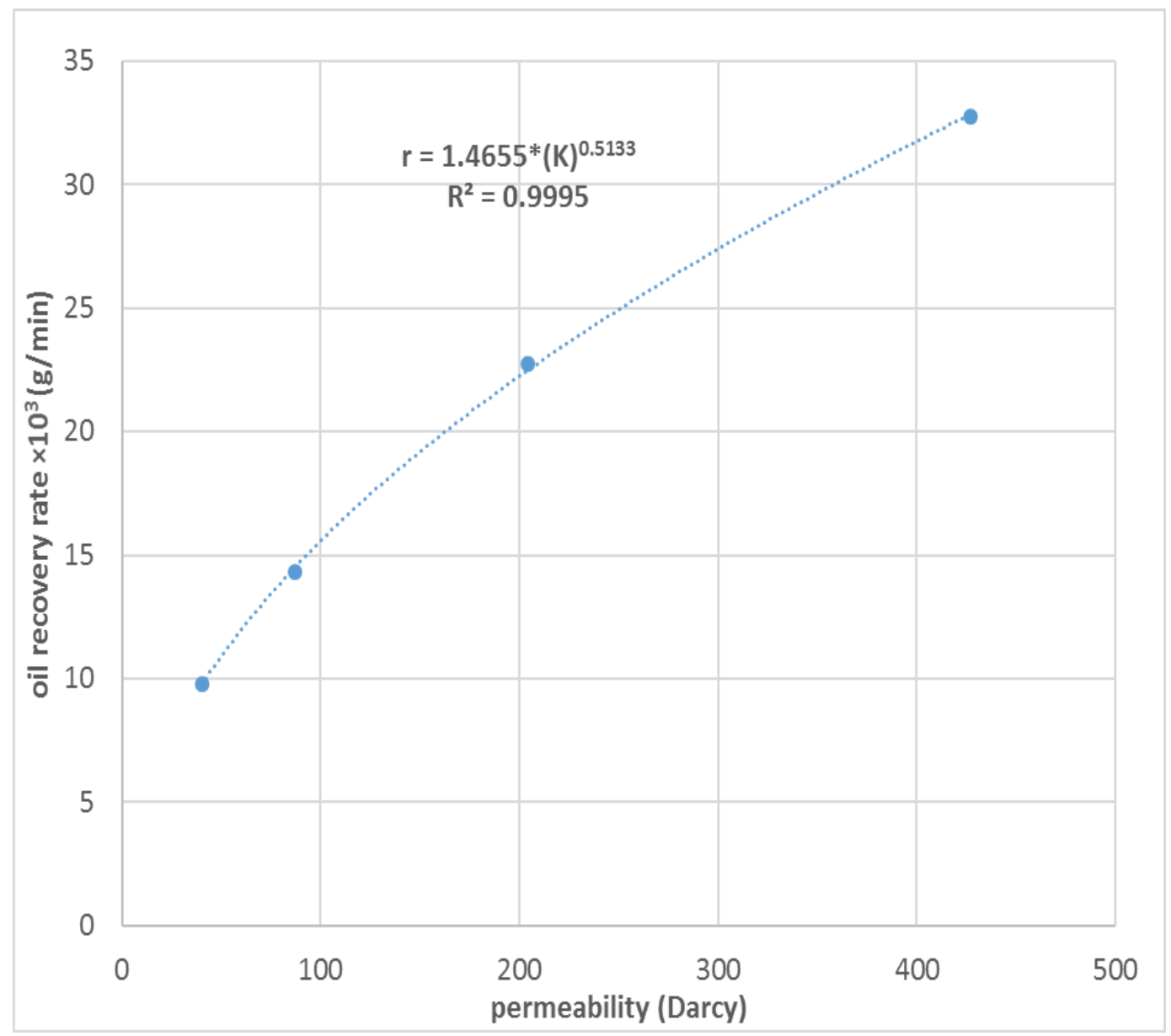

Figure 8: Variation of production rate of air injection with model permeability at $0.514 \mathrm{MPa}$ absolute and $25^{\circ} \mathrm{C}$

It is observed that the correlation between heavy oil production rate and the physical model permeability is neatly fitted by the power function. This result is in close agreement with what is reported in the literature. ${ }^{52,53}$ 


\subsubsection{Effect of Pressure on Heavy Oil Recovery}

Figure 9 shows the effect of air pressure on heavy oil recovery at a constant air temperature of $25^{\circ} \mathrm{C}$ and 204 Darcy model permeability. The results show that the heavy oil recovery increased with air pressure. The overall oil recovery rose from $0.77 \%$ to $1.60 \%$ of the OOIP as pressure was

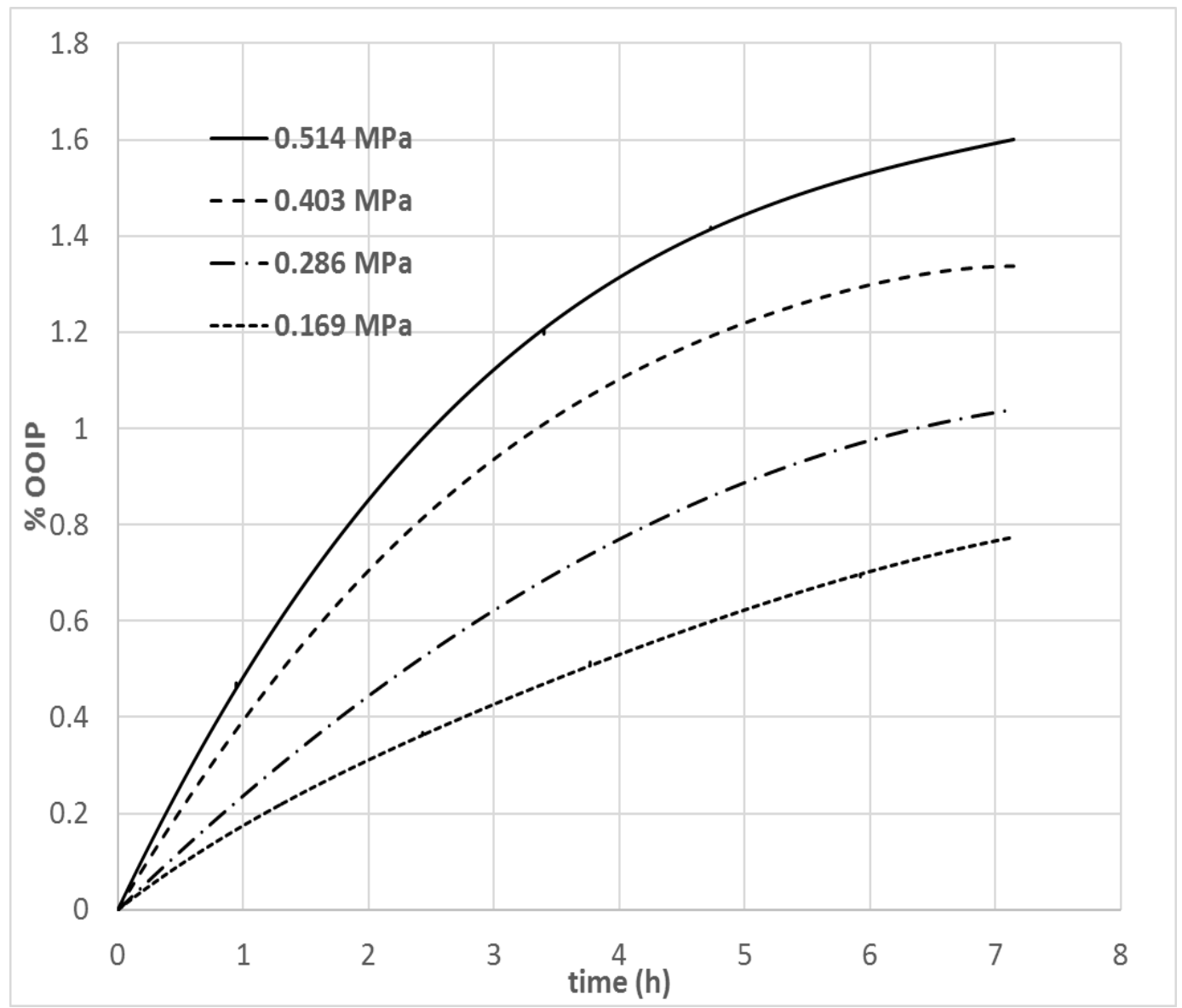

Figure 9: Oil recovery versus time at $25^{\circ} \mathrm{C}$ air temperature, 204 Darcy model permeability, and different air pressures (absolute)

increased from 0.169 to $0.514 \mathrm{MPa}$ absolute. The likely reason is the increase in the solubility of the injected air in heavy oil with pressure. It may be noted that the higher proportion of the air in the oil reduces the oil viscosity and facilitates its drainage from the reservoir. 
A similar experiment was conducted by using propane as a solvent and found that the oil recovery increased with pressure. ${ }^{50}$ Table 7 shows that the mass fraction of air dissolved in the heavy oil increased with pressure.

Table 7: Dissolved air in heavy oil at constant temperature $\left(25^{\circ} \mathrm{C}\right)$ and different pressures

\begin{tabular}{cccc}
$\begin{array}{c}\text { pressure } \\
(\mathrm{MPa} \text { absolute) }\end{array}$ & $\begin{array}{c}\text { dead oil } \\
\text { mass }(\mathrm{g})\end{array}$ & $\begin{array}{c}\text { collected air } \\
\text { mass }(\mathrm{g})\end{array}$ & $\begin{array}{c}\text { dissolved air } \\
\text { mass fraction }\end{array}$ \\
\hline 0.169 & 4.6710 & 0.035010 & 0.0074370 \\
\hline 0.286 & 6.2632 & 0.064030 & 0.010115 \\
\hline 0.403 & 8.0610 & 0.10601 & 0.012979 \\
\hline 0.514 & 9.6402 & 0.15302 & 0.015620
\end{tabular}

\subsubsection{Effect of Temperature on Oil Recovery}

To evaluate the impact of temperature on heavy oil recovery, we performed a number of experiments keeping the air temperature (i) constant, and (ii) periodically varying with time.

\subsubsection{Effect of Air Temperature in Isothermal Experiments}

Four experiments were carried out at constant air temperatures of $25,50,75$ and $90^{\circ} \mathrm{C}$ at $0.514 \mathrm{MPa}$ absolute air pressure with 204 Darcy permeability of the physical model. The results shown in Figure 10 indicate that the final oil recovery increased with temperature to a maximum of $41.2 \%$ of the OOIP at $90^{\circ} \mathrm{C}$.

Apart from the decrease in the oil viscosity with temperature, the increase in the oil recovery may be attributed to the increase in the diffusivity of air (mostly nitrogen) in heavy oil with 
temperature. ${ }^{19}$ Higher diffusivity causes greater uptake of air in the oil and brings about a considerable reduction in the oil viscosity, ${ }^{55}$ which promotes oil recovery. The phenomenon mentioned above is supported by the data in Table 8 . According to the table, the increase in temperature from $25^{\circ} \mathrm{C}$ to $90^{\circ} \mathrm{C}$ and the associated increase in the amount of dissolved air bring

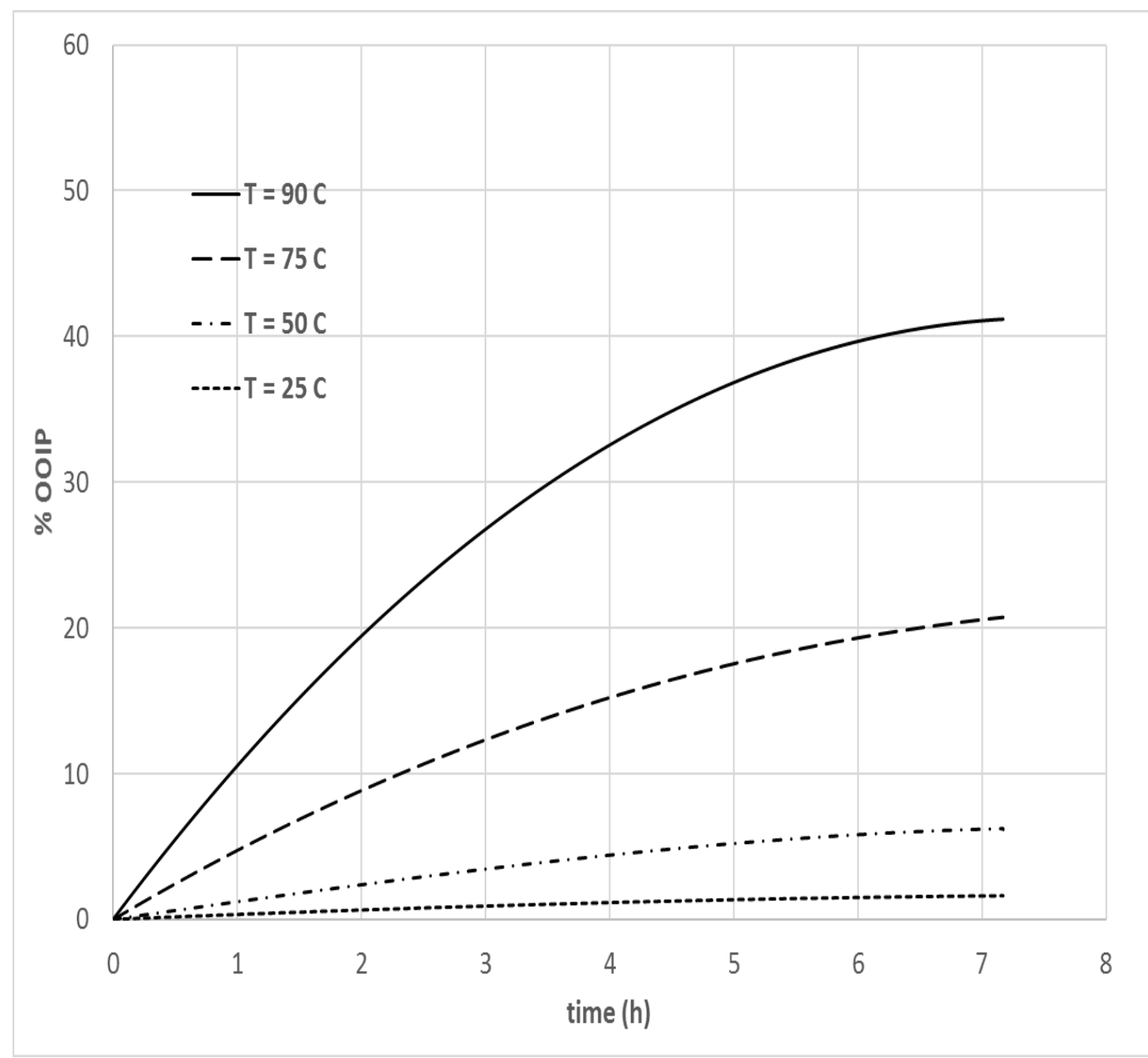

Figure 10: Oil recovery versus time at $0.514 \mathrm{MPa}$ absolute air pressure, 204 Darcy model permeability, and different air temperatures 
Table 8: Live oil viscosity and dissolved air fraction in oil at constant pressure of $0.514 \mathrm{MPa}$ and different temperatures

\begin{tabular}{ccc} 
temperature $\left({ }^{\circ} \mathrm{C}\right)$ & viscosity $(\mathrm{mPa} . \mathrm{s})$ & $\begin{array}{c}\text { dissolved air mass } \\
\text { fraction }\end{array}$ \\
\hline 25 & $11,500.00$ & 0.015601 \\
\hline 50 & 642.00 & 0.061102 \\
\hline 75 & 86.800 & 0.166030 \\
\hline 90 & 11.200 & 0.463010
\end{tabular}

about considerable viscosity reduction from 11,500 to $11.2 \mathrm{mPa} . \mathrm{s}$. The oil viscosity is related to the dissolved air mass fraction as (Figure 11):

$\mu=2.2451(\omega)^{-2.0}$

with the $r^{2}$ value of 0.9998 . A similar trend has been reported earlier for the dissolution of nitrogen and carbon dioxide in heavy oil. ${ }^{55}$ This correlation is used in the modeling simulation in this study. 


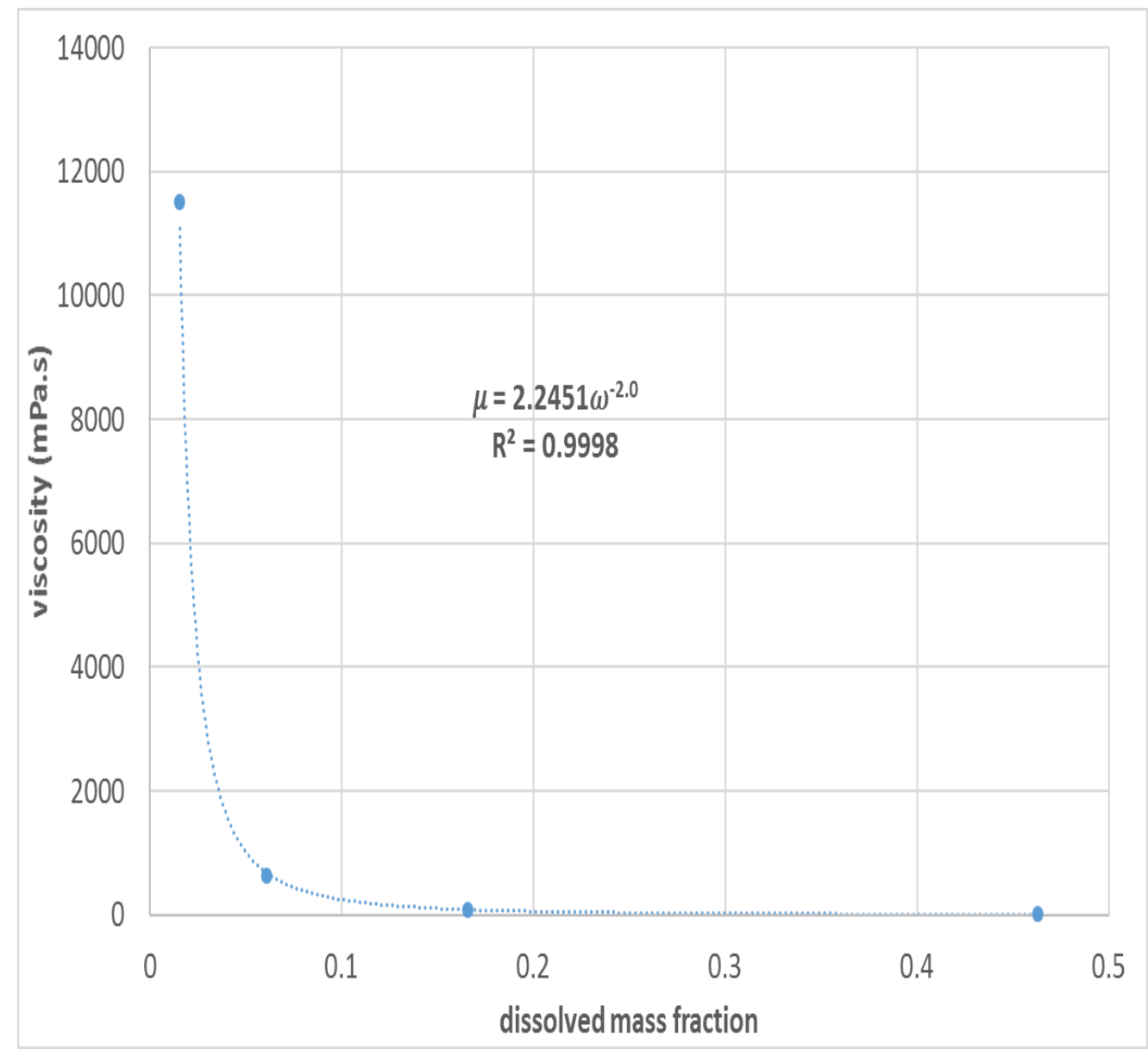

Figure 11: Correlation between viscosity and dissolved air mass fraction at $0.514 \mathrm{MPa}$ absolute and 204 Darcy permeability of the physical model

A comparison of Figures 9 and 10 shows that the increase in air temperature is significantly more conducive to heavy oil recovery than the increase in air pressure. This may be explained by the mass fraction of air found in the produced oil as listed in Tables 7 and 8, respectively, for different pressures and temperatures.

The rise in the dissolved air mass fraction is steeper with temperature than pressure. While the maximum value of the dissolved air mass fraction is 0.0156 at the maximum pressure, that value at the maximum temperature is 0.463 , which is about 30 times higher. The larger amount of air in 
the oil helps to reduce its viscosity to a greater extent and thus enhances its recovery. In addition to reducing the oil viscosity, the increase in temperature is expected to cause the gas-oil mixture to expand and help improve the oil recovery further. ${ }^{3,14}$

\subsubsection{Effect of Variation in the Air Temperature}

To study the effect of variation in the air temperature with time on oil recovery, we performed experiments with periodic variation of air temperature between $50^{\circ} \mathrm{C}$ and $90^{\circ} \mathrm{C}$. Figure 12 shows oil recovery at a constant air temperature of $90^{\circ} \mathrm{C}$ as well as with the periodic temperature variation having an average of $67^{\circ} \mathrm{C}$.

As seen from the figure, the periodic variation of air temperature enhances the recovery in comparison to constant air temperature $\left(90^{\circ} \mathrm{C}\right)$ by $8 \%$ with the final recovery of $44.5 \%$ of the OOIP. Compared to the recovery with constant air temperature, the recovery with the periodic air temperature variation is lower initially but eventually surpasses the former after six $\mathrm{h}$ at a "crossover point" as shown in the figure.

To further examine the impact of the periodic temperature variations with a higher minimum temperature, we performed experiments with periodic variation of air temperature between $75^{\circ} \mathrm{C}$ and $90^{\circ} \mathrm{C}$ as shown in Figure 13. As observed from the figure, in comparison to constant air temperature, the periodic air temperature variation enhanced oil recovery even more by $17.6 \%$ with the final value of $48.4 \%$ of the OOIP. 
It is interesting to note that the oil recovery corresponding to the periodic air temperature crossed over the constant temperature curve at a much earlier time (about four h earlier) than that in Figure 12. This may be attributed to the overall higher average air temperature of $78^{\circ} \mathrm{C}$ in Figure 13 compared to $67^{\circ} \mathrm{C}$ in Figure 12.

The increase in oil recovery with periodic air temperature may be ascribed to the corresponding periodic change in surface temperature of the physical model and the associated periodic reversal in the temperature gradient as well as the concentration gradient of air (through similar diffusivity and solubility changes ${ }^{19,42}$ ) within the model. This alteration of gradients boosts dispersion and leads to better mixing of air with the oil, thereby promoting its recovery. ${ }^{42}$ 


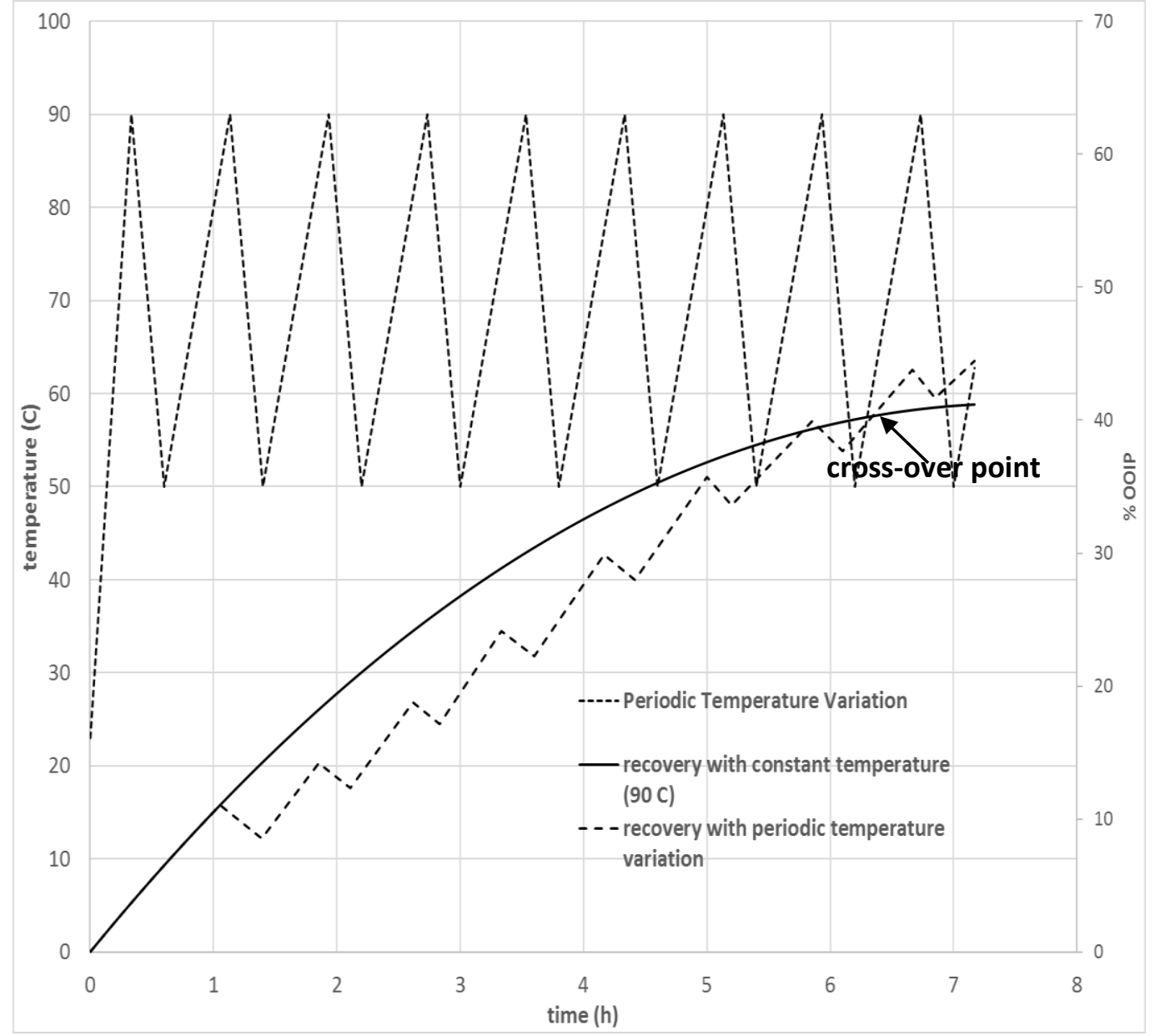

Figure 12: Oil recovery versus time at $0.514 \mathrm{MPa}$ absolute air pressure, 204 Darcy model permeability, and constant $\left(90^{\circ} \mathrm{C}\right)$ as well as periodically varying air temperature in the range, 50 $90^{\circ} \mathrm{C}$. 


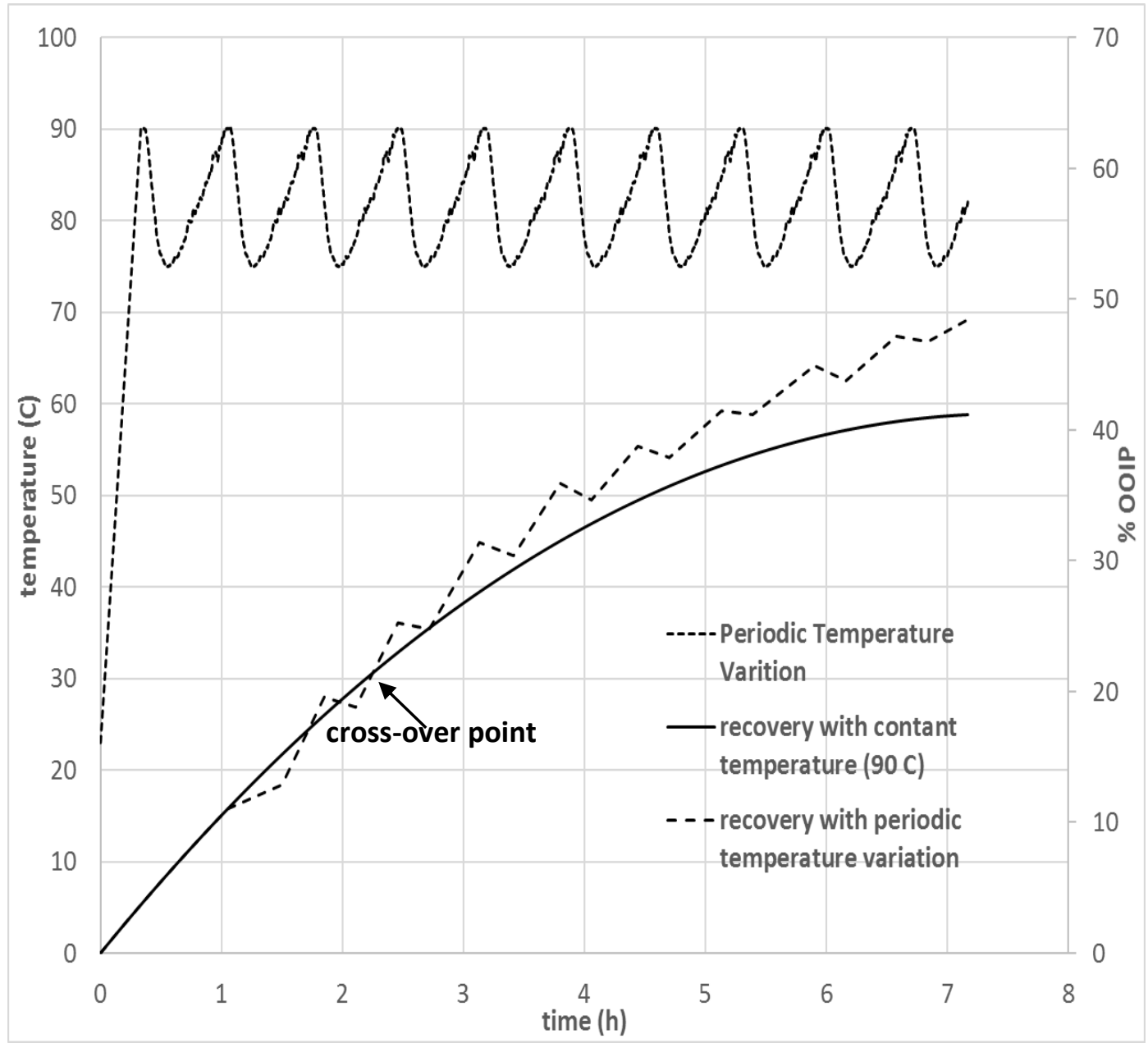

Figure 13: Oil recovery versus time at $0.514 \mathrm{MPa}$ absolute air pressure, 204 Darcy model permeability, and constant $\left(90^{\circ} \mathrm{C}\right)$ as well as periodically varying air temperature in the range, 75 $90^{\circ} \mathrm{C}$

It is important to note that to enable a better understanding of the impact of periodic temperature variation on heavy oil recovery, instantaneous recovery is plotted against cumulative recovery at the constant temperature of $90^{\circ} \mathrm{C}$ in Figures 12 and 13.

\subsubsection{Effect of Varying Temperature with Different Permeabilities}

The effect of periodic temperature variations between $75^{\circ} \mathrm{C}$ to $90^{\circ} \mathrm{C}$ on oil recovery was examined for different permeability values of 40, 87 and 427 Darcy. Figures 14-16 show the oil recovery 
versus time with periodic air temperature variations compared to a constant temperature $\left(90^{\circ} \mathrm{C}\right)$ at different physical model permeability. The results indicate that in all experiments, the oil recovery increased in periodic temperature variations compared to that at the constant temperature. As the permeability rose from 40 to 427 Darcy, the OOIP improved from $14.8 \%$ to $18.6 \%$, to the

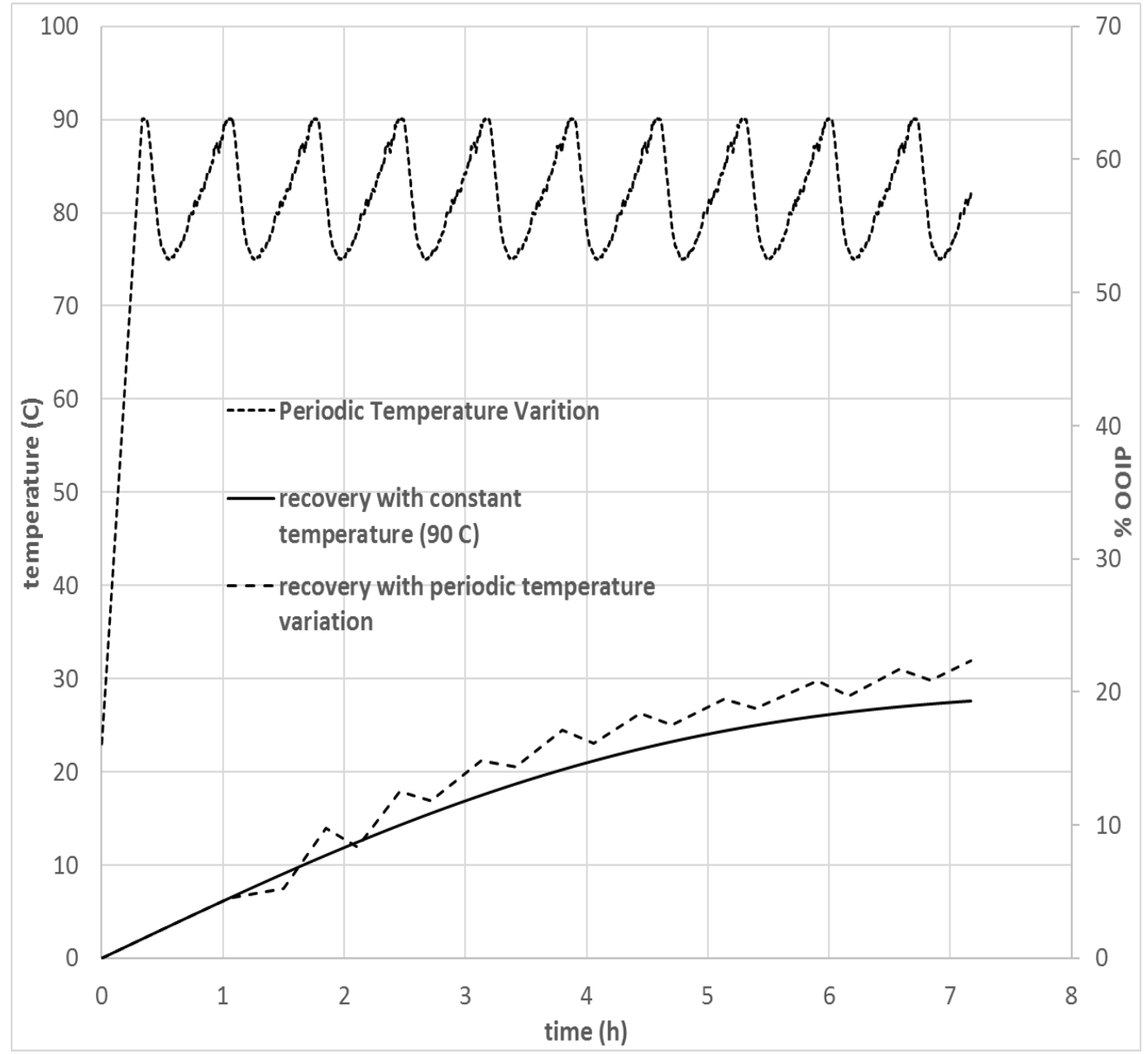

Figure 14: Oil recovery versus time at $0.514 \mathrm{MPa}$ absolute air pressure, constant $\left(90^{\circ} \mathrm{C}\right)$ as well as periodically varying air temperature in the range, $75-90^{\circ} \mathrm{C}$, and 40 Darcy model permeability

maximum of $69.1 \%$ OOIP. The oil recovery curves corresponding to periodic temperatures are observed to cross over the constant temperature oil recovery curves at about $2.2 \mathrm{~h}$, i.e. after one full cycle of temperature variation with time. 
Even though the permeability of the physical model increased tremendously, the oil recovery increased marginally. These results indicate that periodic temperature variation has a significantly influence on oil recovery than permeability.

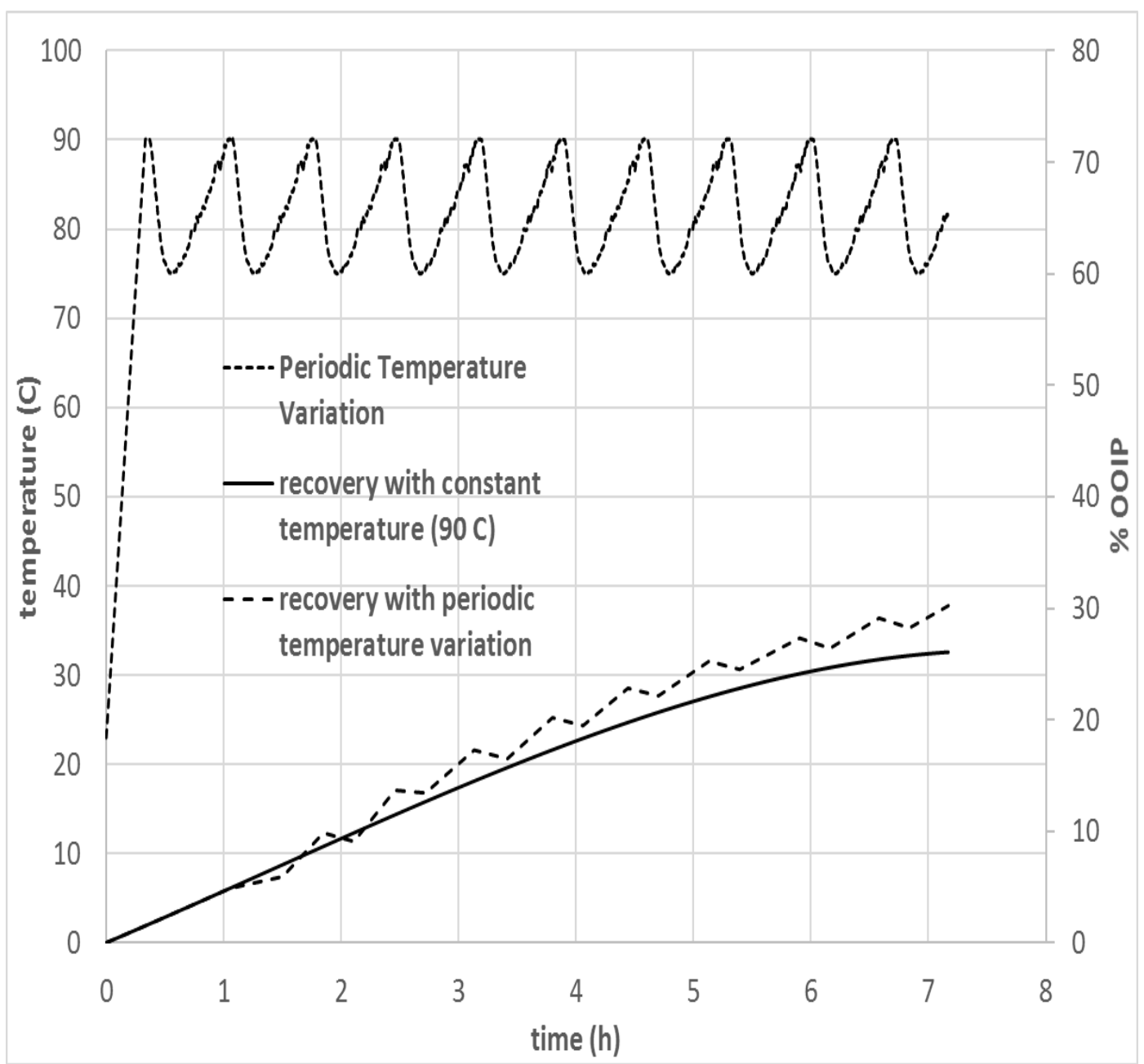

Figure 15: Oil recovery versus time at $0.514 \mathrm{MPa}$ absolute air pressure, constant $\left(90^{\circ} \mathrm{C}\right)$ as well as periodically varying air temperature in the range, $75-90^{\circ} \mathrm{C}$, and 87 Darcy model permeability 


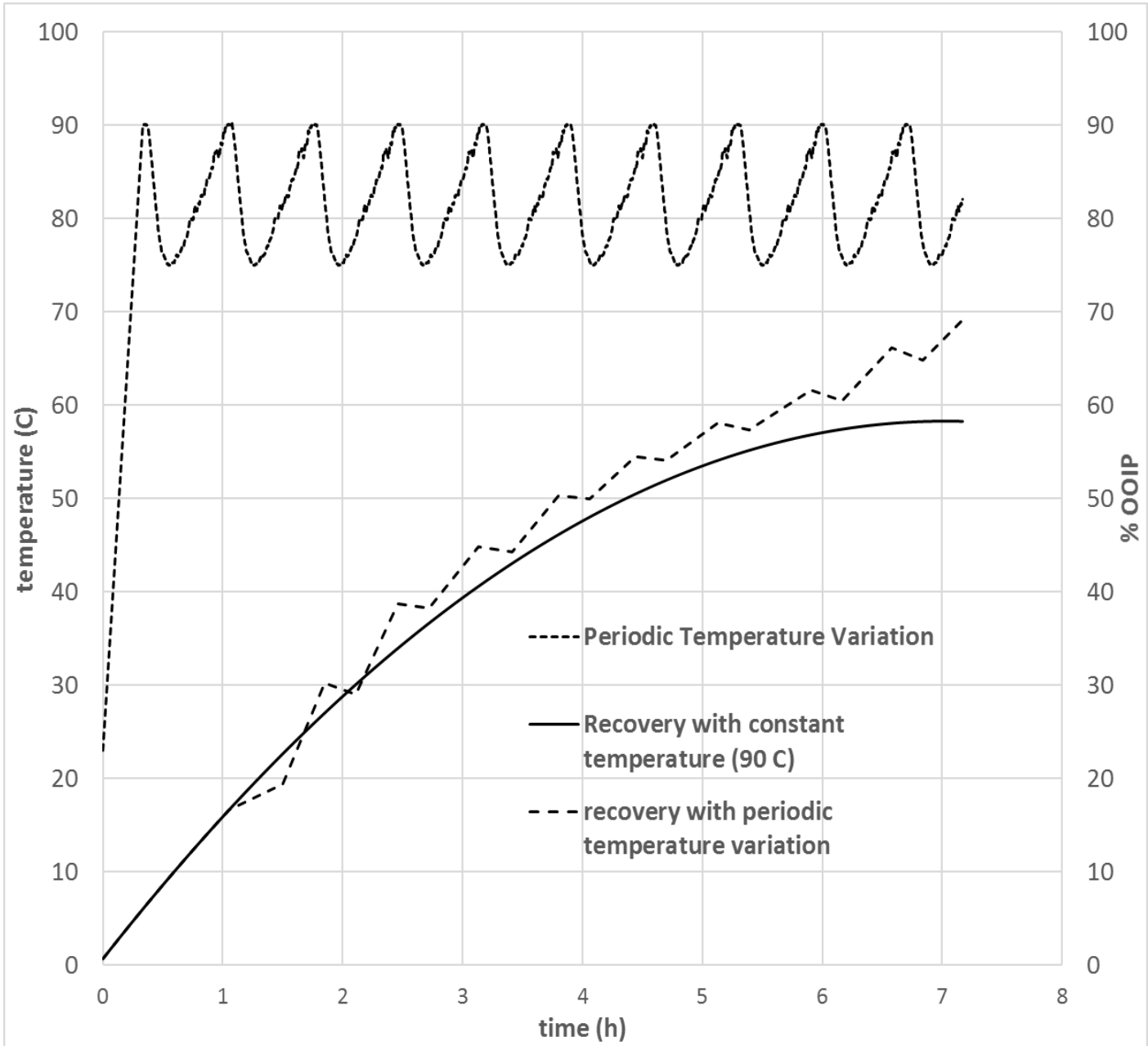

Figure 16: Oil recovery versus time at $0.514 \mathrm{MPa}$ absolute air pressure, constant $\left(90^{\circ} \mathrm{C}\right)$ as well as periodically varying air temperature in the range, $75-90^{\circ} \mathrm{C}$, and 427 Darcy model permeability

In summary, the results above indicate that the heavy oil recovery up to $69.1 \%$ of the OOIP can be achieved by utilizing freely available air under moderate temperature and pressure conditions in a non-reactive environment. The results at $0.514 \mathrm{MPa}$ air pressure show a considerable improvement in heavy oil recovery from $1.6 \%$ of the OOIP at $25^{\circ} \mathrm{C}$ air temperature to $69.1 \%$ of the OOIP with the periodic variation of air temperature between $75^{\circ} \mathrm{C}$ and $90^{\circ} \mathrm{C}$.

These findings are therefore very promising for heavy oil recovery using freely available air at low temperatures and pressures. 


\subsubsection{Absence of Chemical Reactions}

As previously mentioned, oxygen in the air reacts with hydrocarbons during in-situ combustion and generates carbon dioxide, water, and energy. To determine whether any reaction took place during the experiments of this study, we analyzed several gas effluent samples (using Perkin Elmer GCMS/TCD equipment for gas analysis) from experiments carried out at different constant temperatures, $0.514 \mathrm{MPa}$ absolute air pressure with 204 Darcy permeability of the physical model. In all cases, the air composition was found to be the same as that of the fresh air. Table 9 presents the air composition for the experiment at $90^{\circ} \mathrm{C}$. Furthermore, no sudden increase in the reservoir temperature was observed throughout the experiments. Lastly, the viscosity of the live oil decreased with temperature. As a result, it may be concluded that no chemical reactions occurred in the experiments done in this study. Lastly, the percentage errors between the component of air analysis from the repeated experiments were found to be in the range of $0.2-0.5 \%$.

Table 9: Analysis of air for the experiment done at $90^{\circ} \mathrm{C}$

\begin{tabular}{ccc}
\multirow{2}{*}{$\begin{array}{c}\text { Component } \\
\text { of air }\end{array}$} & \multicolumn{2}{c}{ mass fraction } \\
\cline { 2 - 3 } fresh air & effluent air \\
\hline oxygen & 0.21201 & 0.21201 \\
\hline nitrogen & 0.77502 & 0.77502 \\
\hline $\begin{array}{c}\text { carbon } \\
\text { dioxide }\end{array}$ & $4.6011 \times 10^{-4}$ & $4.6011 \times 10^{-4}$ \\
\hline
\end{tabular}

Until now, we have proved the concept that the heavy oil recovery can be achieved by utilizing freely available air under moderate temperature and pressure conditions in a non-reactive environment. In the following sections, the results of an extensive simulation study of atmospheric air injection will be described and discussed. 


\subsection{Optimal Control Results}

One of the primary objectives of this study is to formally develop and utilize a tool to obtain optimal control policy. As mentioned earlier, experiments were conducted using laboratory-scale physical model at different pressures $(0.169,0.286,0.403$ and $0.514 \mathrm{MPa}$ absolute) and temperatures in the range, $25-90^{\circ} \mathrm{C}$. Reservoirs of four different permeabilities $(40,87,204$, and 427 Darcy) are used in the experiments.

In this computational simulation, some parameters were measured experimentally, such as live oil viscosity, live oil density, the interfacial air temperature, and the interfacial mass fraction to increase the confidence in the results of the computational simulation.

\subsubsection{Execution and Formalization of the Concept}

To formalize the concept that the variation of air interfacial temperature versus time enhances the heavy oil recovery in our lab-scale system, we needed to accomplish the following steps:

1. theoretically determine optimal policy for air interfacial temperature versus time, which maximize heavy oil recovery

2. experimentally validate the optimal policy

It is important to note that the first step requires the knowledge on the correlation between dispersion coefficient and concentration and temperature that is used in the mathematical model of the process. 


\subsubsection{Determination of Dispersion Coefficient}

As previously mentioned, the gas dispersion coefficient is an important property used in the mathematical model utilized in the optimal control of gas EOR. The dispersion coefficient of air was determined using the technique developed earlier. This technique uses optimal control to determine optimal solvent dispersion versus concentration function. ${ }^{46}$

The optimal $[D(\omega, T)]$ of atmospheric air is plotted in Figure 17 at the injection pressure of 0.514 $\mathrm{MPa}$, temperature of $25^{\circ} \mathrm{C}$, and physical model permeability of 204 Darcy. It shows that the optimally determined $[D(\omega, T)]$ rises to a maximum value, and then drops toward the end. The highest value of atmospheric air dispersion is $2.5310 \times 10^{-5}\left(\mathrm{~m}^{2} / \mathrm{s}\right)$ at the corresponding air mass fraction of 0.33600 .

It is observed that the calculated dispersion value is about $37.47 \%$ lower compared to that with propane. ${ }^{46}$ This is due to the fact that propane has a higher diffusivity in comparison to atmospheric air, but this is still very encouraging given that air is freely available while hydrocarbon solvents, such as propane are costly and their use for oil recovery is almost impractical at present. 


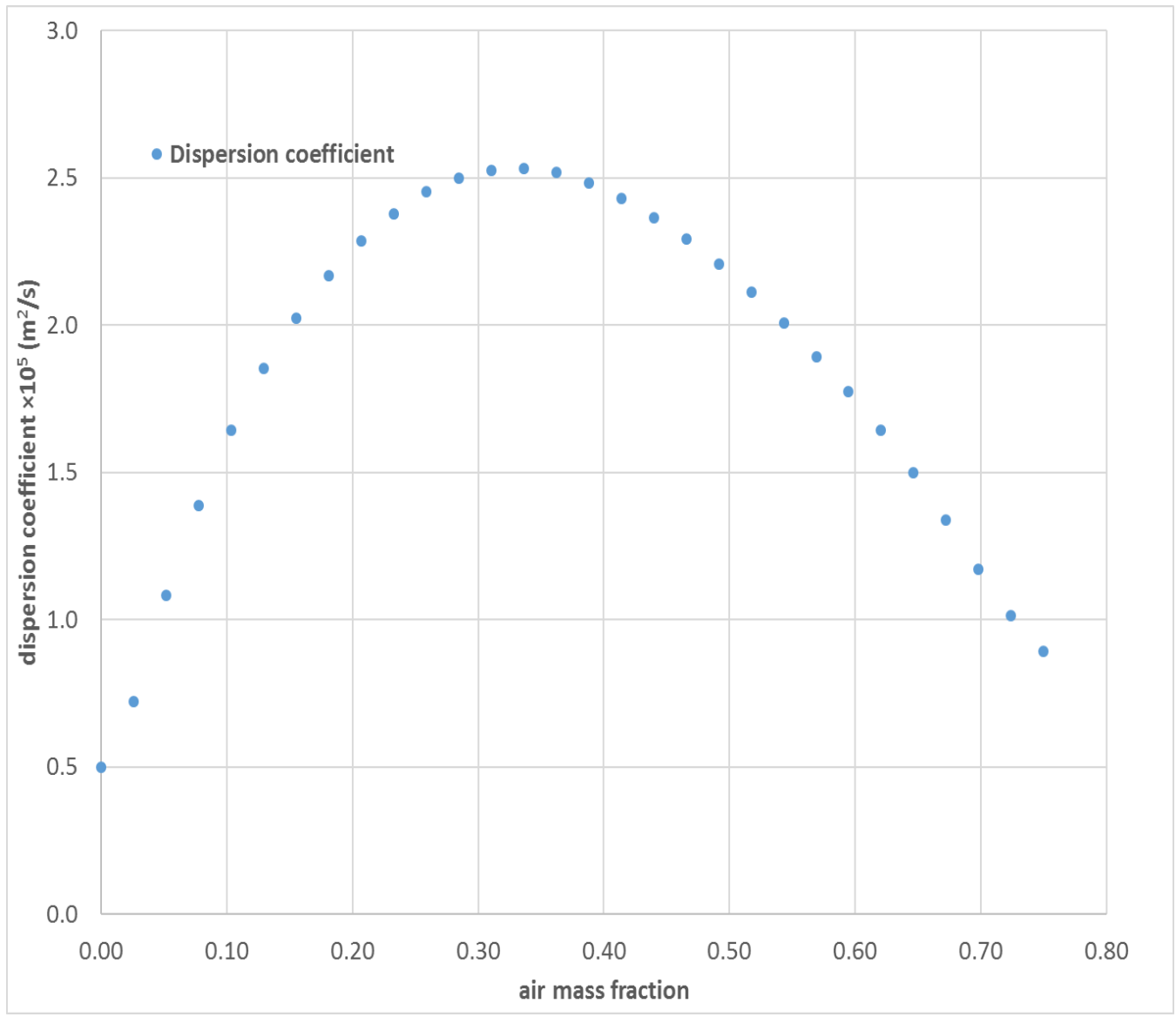

Figure 17: Dispersion coefficient function of air injection in heavy oil (Pressure 0.514 $\mathrm{MPa}$; Temperature $25^{\circ} \mathrm{C}$; Permeability 204 Darcy)

The change in optimal dispersion coefficient with air mass fraction may be due to the fact that initially when higher concentration gradients are present in the heavy oil, the dispersion of air molecules is higher. It decreases later on with a gradual reduction in the concentration gradients as more and more air molecules enter the porous medium because of dispersion. When this happens, the motion of air molecules is restricted by their abundance, thus decreasing the overall air dispersive flux. When the air diffusion flux and the convective flux are at their maximum, the dispersion coefficient value gains the highest value. Thus, at some intermediate stage, the dispersion coefficient is at its maximum. ${ }^{49}$ 
Moreover, under the set operational conditions, the objective functional decreased monotonically to the minimum as shown in Figure 18. The change was significant at the beginning, but the rate of improvement slowed down at final iterations. The final optimal function $[D(\omega, T)]$ was obtained in 31 iterations after which no further increase was observed.

We also determined the dispersion coefficient at different air temperatures of $50^{\circ} \mathrm{C}, 75^{\circ} \mathrm{C}$, and $90^{\circ} \mathrm{C}$. Figure 19 shows the optimally obtained dispersion coefficient of atmospheric air at different temperatures of $25^{\circ} \mathrm{C}, 50^{\circ} \mathrm{C}, 75^{\circ} \mathrm{C}$, and $90^{\circ} \mathrm{C}$. Similar to Figure 17, the value of $[D(\omega, T)]$ with respect to $50^{\circ} \mathrm{C}, 75^{\circ} \mathrm{C}$, and $90^{\circ} \mathrm{C}$ increase to a maximum value, and then drops toward the end. Lastly, the plot of maximum dispersion coefficient at different temperatures is shown in Figure 20.

It is important to note that the optimal dispersion coefficient values at different temperatures were used to compute the optimal control policy for atmospheric air injection versus time in this process. 


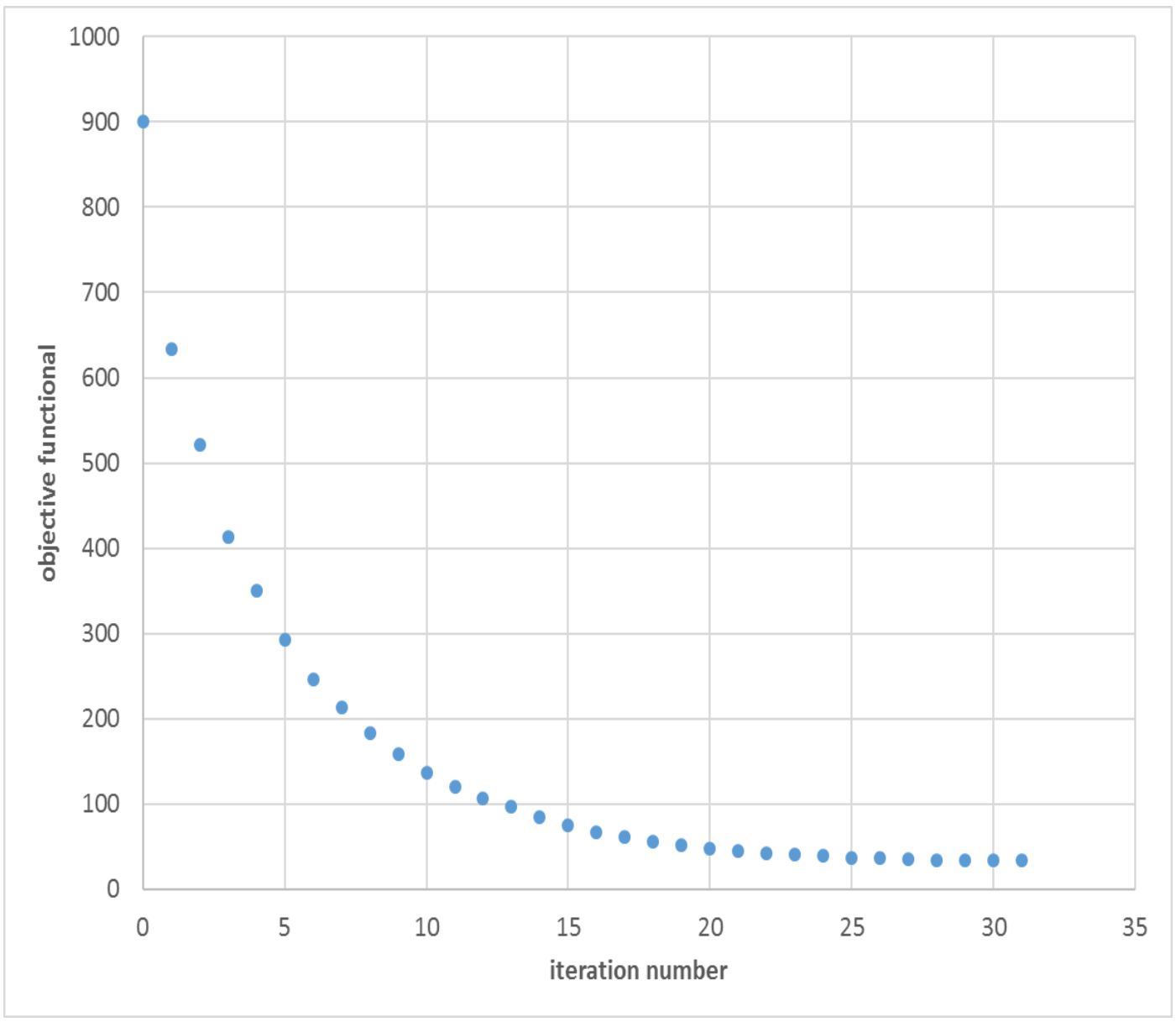

Figure 18: Objective functional versus iteration number for dispersion coefficient at air pressure $0.514 \mathrm{MPa}$, temperature $25^{\circ} \mathrm{C}$, and permeability 204 Darcy 


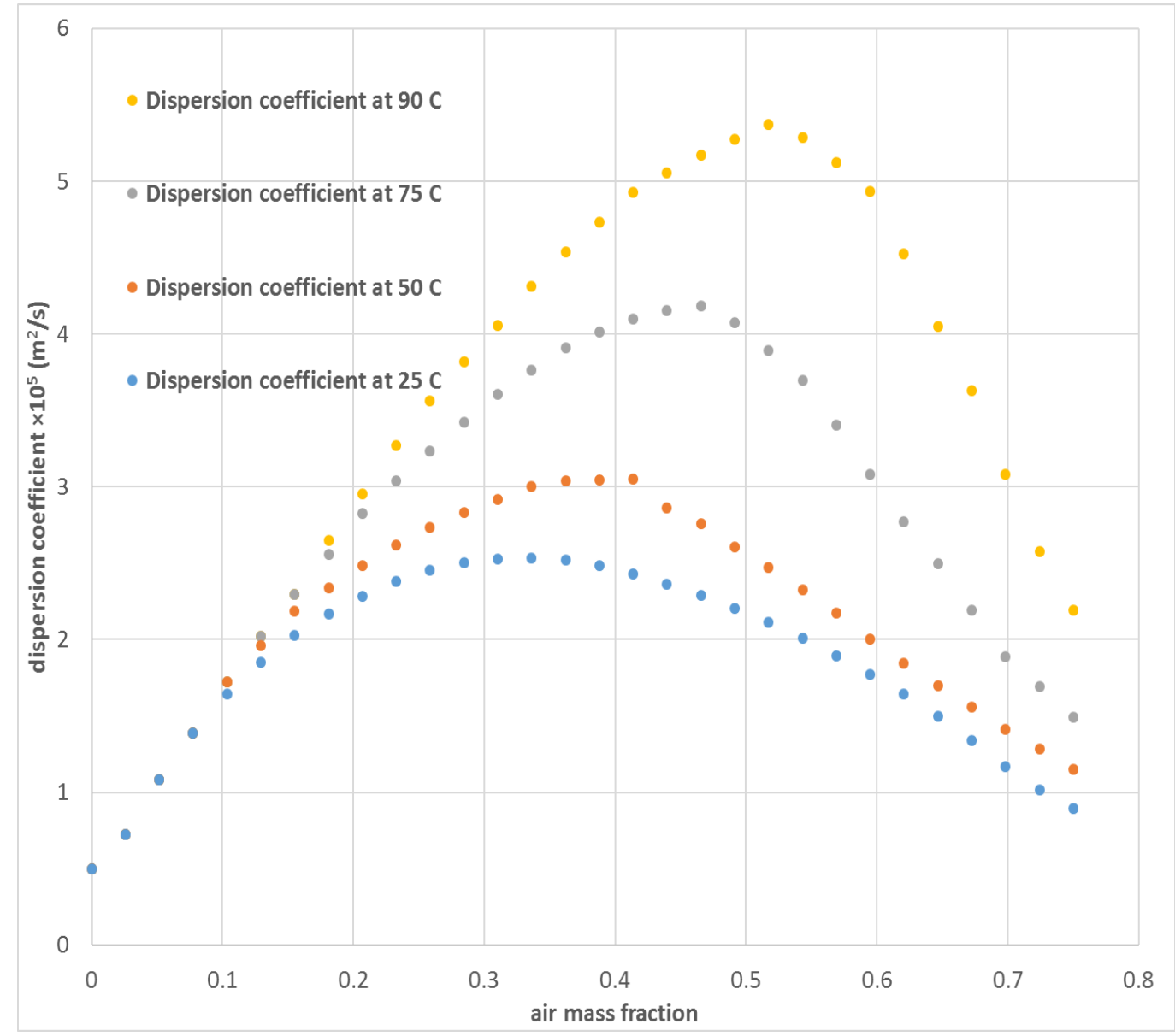

Figure 19: Dispersion coefficient function of air injection in heavy oil at different temperatures $\left(25^{\circ} \mathrm{C}, 50^{\circ} \mathrm{C}, 75^{\circ} \mathrm{C}\right.$, and $90^{\circ} \mathrm{C}$ ) (Pressure $0.514 \mathrm{MPa}$; Permeability 204 Darcy) 


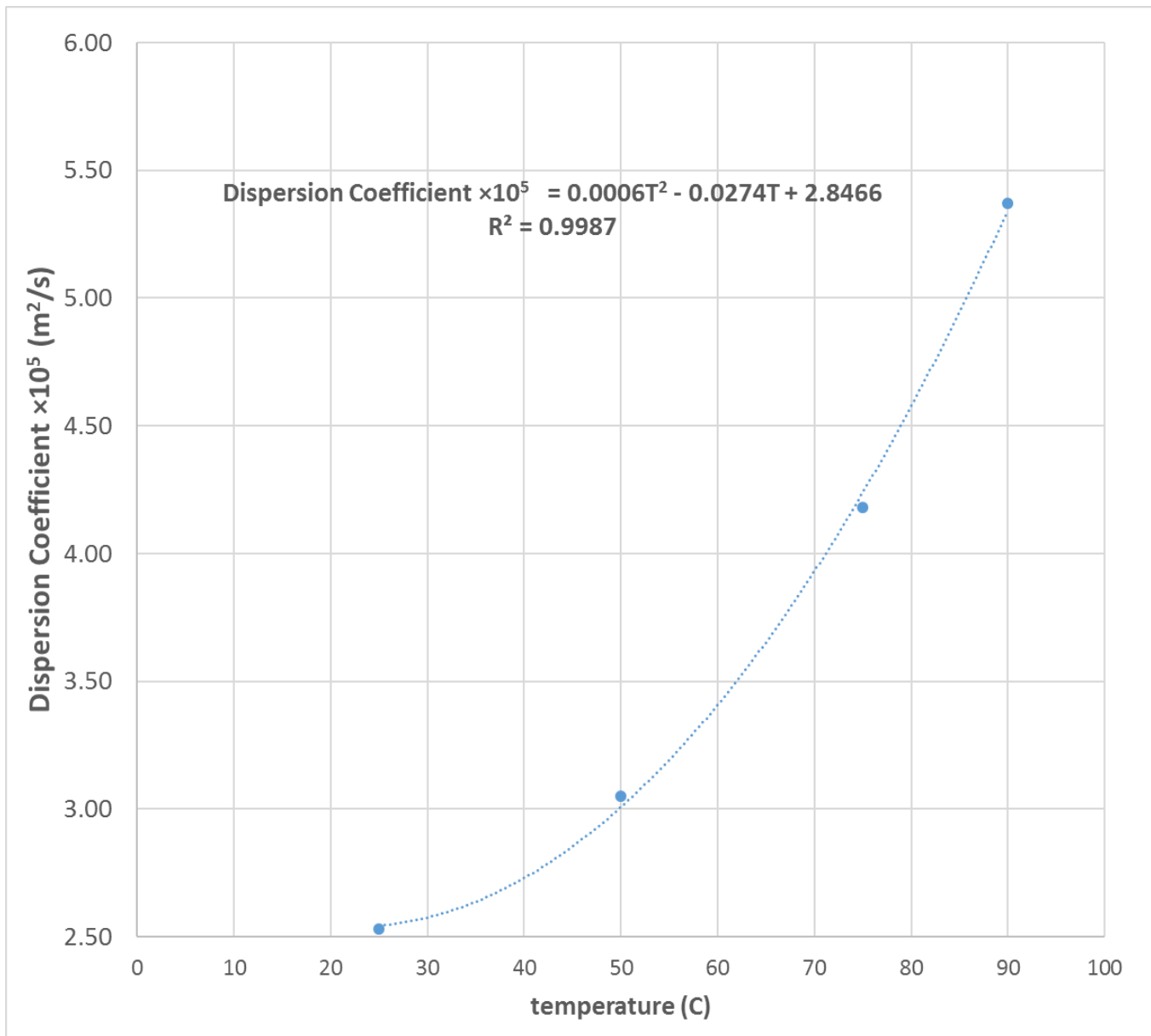

Figure 20: Maximum dispersion coefficient of air at different temperatures

\subsubsection{Optimal Control Policy for Air Interfacial Temperature}

The optimal control policy was obtained from the optimal control algorithm (See Section 4.6), where the interfacial temperature versus time, $\left[T_{\text {int }}(t)\right]$ is the control function of the process.

For a given set of operational conditions, the optimal air interfacial temperature policy is associated with the maximum heavy oil recovery calculated for the atmospheric air gas EOR. This policy is expected to yield enhanced experimental heavy oil recovery in agreement with a calculated 
counterpart. This agreement is cross-checked in the experimental validation in the second step as laid out earlier in Section 5.2.1.

Figure 21 shows the air interfacial temperature versus time policy for different iteration based on an initial guess of the constant interfacial temperature of $90^{\circ} \mathrm{C}$ at the pressure of $0.514 \mathrm{MPa}$ and physical media permeability of 204 Darcy for 165 minutes of operational time. It can be seen that as iterative increases, the interfacial temperature after 1-hour periodically starts decreasing from $90^{\circ} \mathrm{C}$ to $82^{\circ} \mathrm{C}$. It results in an iterative increasing of the objective functional companies to the maximum improvement in $\left[T_{\mathrm{int}}(t)\right]$. The objective functional increases monotonically to the highest value of 175.55 (Figure 22). The change is significant at the beginning, but the rate of improvement slows down at final iteration. The final optimal function $\left[T_{\text {int }}(t)\right]$ is obtained in 15 iterations. Table 10 lists the parameters used in the simulation of the mathematical model for the physical model of 204 Darcy permeability and the pressure of $0.514 \mathrm{MPa}$.

Lastly, the objective functional improvement over the constant temperature $\left(90^{\circ} \mathrm{C}\right)$ is about $20.66 \%$. Finally, we can see that after 60 min the air production with oscillations in interfacial temperature is much better than the steady maximum interfacial temperature (Figure 21). As it was stated in preliminary results, this result may be ascribed to the corresponding periodic change in surface temperature of the physical model and the associated periodic reversal in the temperature gradient as well as the concentration gradient of air (through corresponding diffusivity and solubility changes ${ }^{19,40}$ ) within the physical model. This alteration of gradients boosts dispersion and leads to better mixing of air with the oil, thereby promoting its recovery. ${ }^{42}$ 
Table 10: Simulation model parameters of the mathematical model (Pressure 0.514 MPa, 204 Darcy permeability)

\begin{tabular}{|c|c|c|}
\hline parameter & description & value \\
\hline$K_{r}$ & relative permeability & 1 \\
\hline$K$ & permeability & $2.0130 \times 10^{-6}\left(\mathrm{~cm}^{2}\right)$ \\
\hline$\rho$ & live oil density & $0.78450\left(\mathrm{~g} / \mathrm{cm}^{3}\right)$ \\
\hline$g$ & gravity & $3531600\left(\mathrm{~cm} / \mathrm{min}^{2}\right)$ \\
\hline$\varnothing$ & porosity & 0.38 \\
\hline$\mu_{o}$ & $\begin{array}{c}\text { live oil viscosity } \\
\text { coefficient }\end{array}$ & 1.3471 (g/cm.min) \\
\hline$k$ & $\begin{array}{c}\text { thermal conductivity } \\
\text { coefficient }\end{array}$ & $\begin{array}{c}0.055800 \\
\left(\mathrm{~J} / \mathrm{cm} \cdot \mathrm{min} .{ }^{\circ} \mathrm{C}\right)^{*}\end{array}$ \\
\hline$C_{p}$ & specific heat capacity & $2.1300\left(\mathrm{~J} / \mathrm{g} .{ }^{\circ} \mathrm{C}\right)^{* *}$ \\
\hline$N_{r}$ & $\begin{array}{c}\text { number of nodes in } \mathrm{r} \\
\text { direction }\end{array}$ & 20 \\
\hline$N_{z}$ & $\begin{array}{c}\text { number of nodes in } \mathrm{z} \\
\text { direction }\end{array}$ & 10 \\
\hline$h_{i}$ & initial step size & $10^{-5}$ \\
\hline$h_{\max }$ & maximum step size & 1 \\
\hline esp & accuracy of integration & $10^{-6}$ \\
\hline$R$ & radius of physical model & $3(\mathrm{~cm})$ \\
\hline$Z$ & height of physical model & $25(\mathrm{~cm})$ \\
\hline ITMAX & $\begin{array}{c}\text { maximum number of } \\
\text { iteration }\end{array}$ & 200 \\
\hline STPMX & $\begin{array}{l}\text { maximum step length for } \\
\text { line search }\end{array}$ & $10^{-2}$ \\
\hline
\end{tabular}

*, ** data provided from Saskatchewan Research Council, Alberta, Canada 


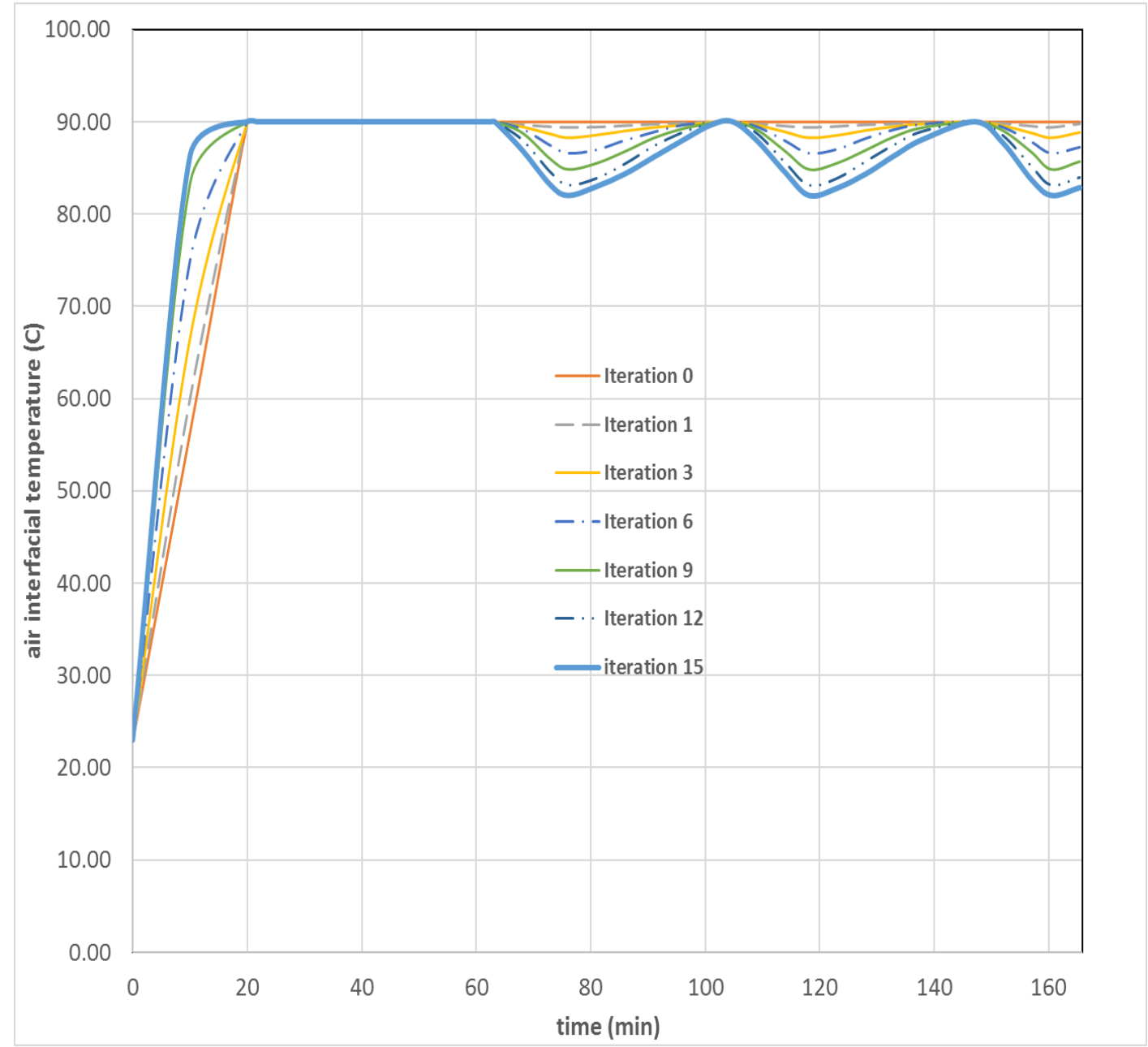

Figure 21: The interfacial air temperature $T_{\text {int }}(t)$ at different iterations on an initial guess of the constant interfacial temperature of $90^{\circ} \mathrm{C}$ 


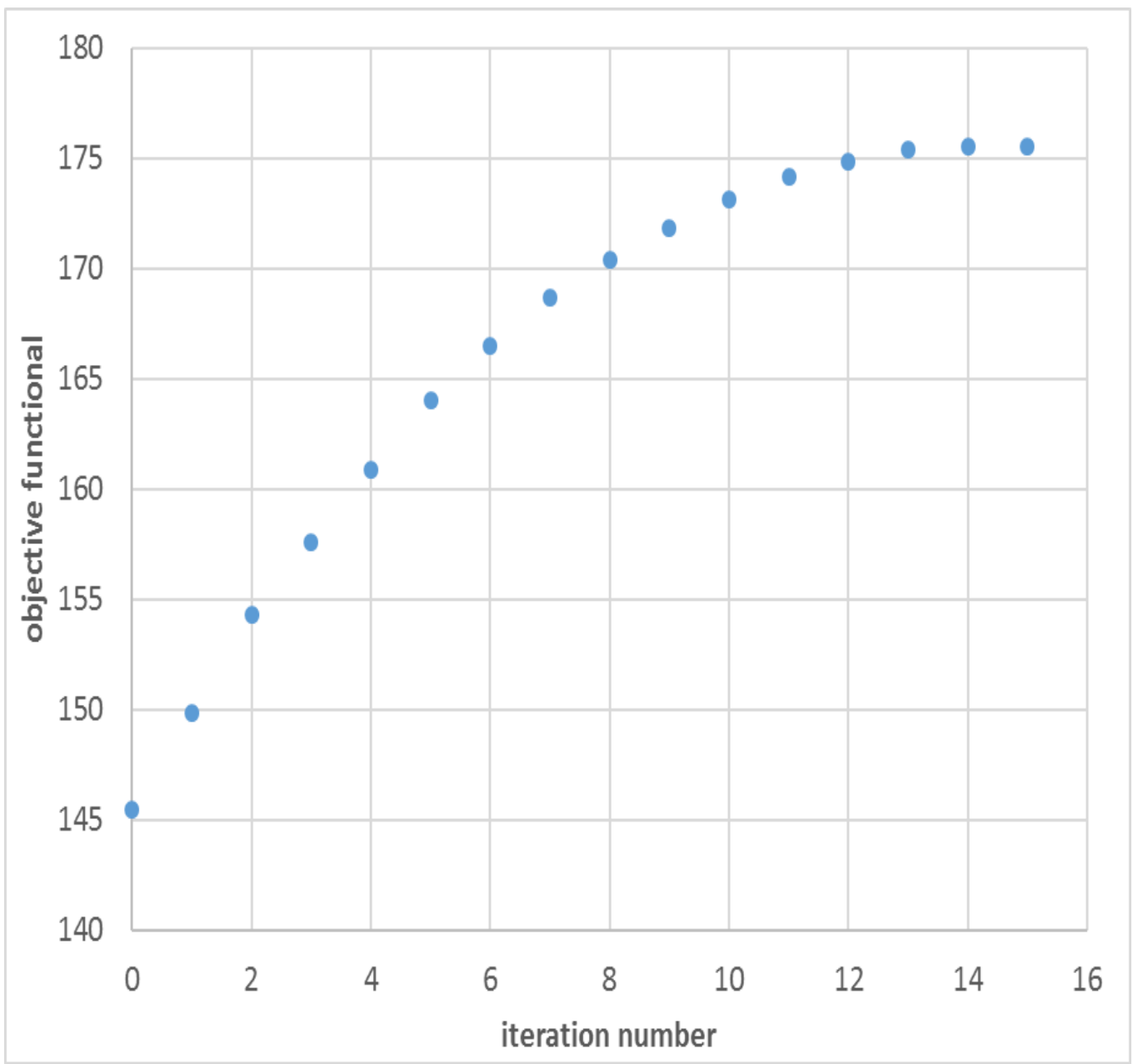

Figure 22: Objective functional versus iteration number for air injection (204 Darcy and Pressure $0.514 \mathrm{MPa})$

For a better understanding the impact of initial $T_{\text {int }}$ guess, we run the computation with a different initial guess to investigate its effect on the optimal objective functional. For a second trial, we have initiated a periodic temperature variation between $90^{\circ} \mathrm{C}$ to $75^{\circ} \mathrm{C}$ (Similar to Figure 13) as our initial guess of interfacial temperature $\left[T_{\mathrm{int}}(t)\right]$. Figure 23 shows the air interfacial temperature versus time policy on an initial guess of periodic temperature variation. According to the figure, for the first hour, the $\left[T_{\text {int }}(t)\right]$ iteratively increased to $90^{\circ} \mathrm{C}$, while after one hour the $\left[T_{\text {int }}(t)\right]$ iteratively periodically increased from $75^{\circ} \mathrm{C}$ to $82^{\circ} \mathrm{C}$. This resulted in an iterative increasing of the objective functional to the maximum value of 175.55 . Also, Figure 24 shows the objective function versus 
the iteration number with the highest value of 175.55 . The final optimal function $\left[T_{\operatorname{int}}(t)\right]$ was obtained in 12 iterations.

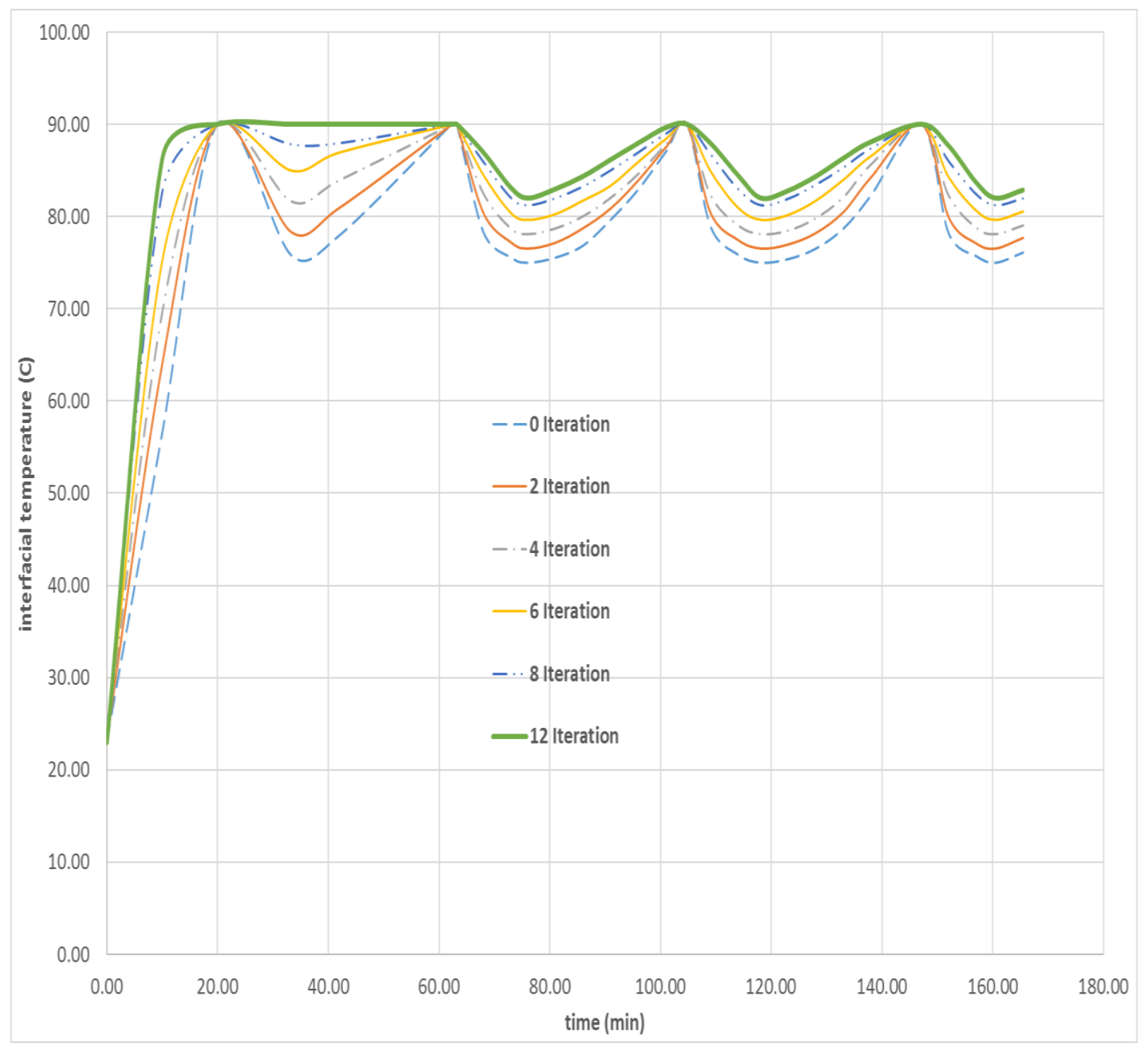

Figure 23: The interfacial solvent temperature $T_{\text {int }}(t)$ at different iterations on initial guess of periodic temperature variation between $90^{\circ} \mathrm{C}$ and $75^{\circ} \mathrm{C}$ after $20 \mathrm{~min}$

It is interesting to note that the comparison of Figures 21 and 23 shows that there is no oscillation in the optimal interfacial temperature before $60 \mathrm{~min}$. This indicates that the optimal oil production by that time was achieved at the maximum temperature of $90^{\circ} \mathrm{C}$. To double-check this observation, we performed a computational run for 60 min only at an initial guess of periodic temperature variation between $90^{\circ} \mathrm{C}$ and $75^{\circ} \mathrm{C}$. We observed the same pattern (Figure 23) that as iterative 


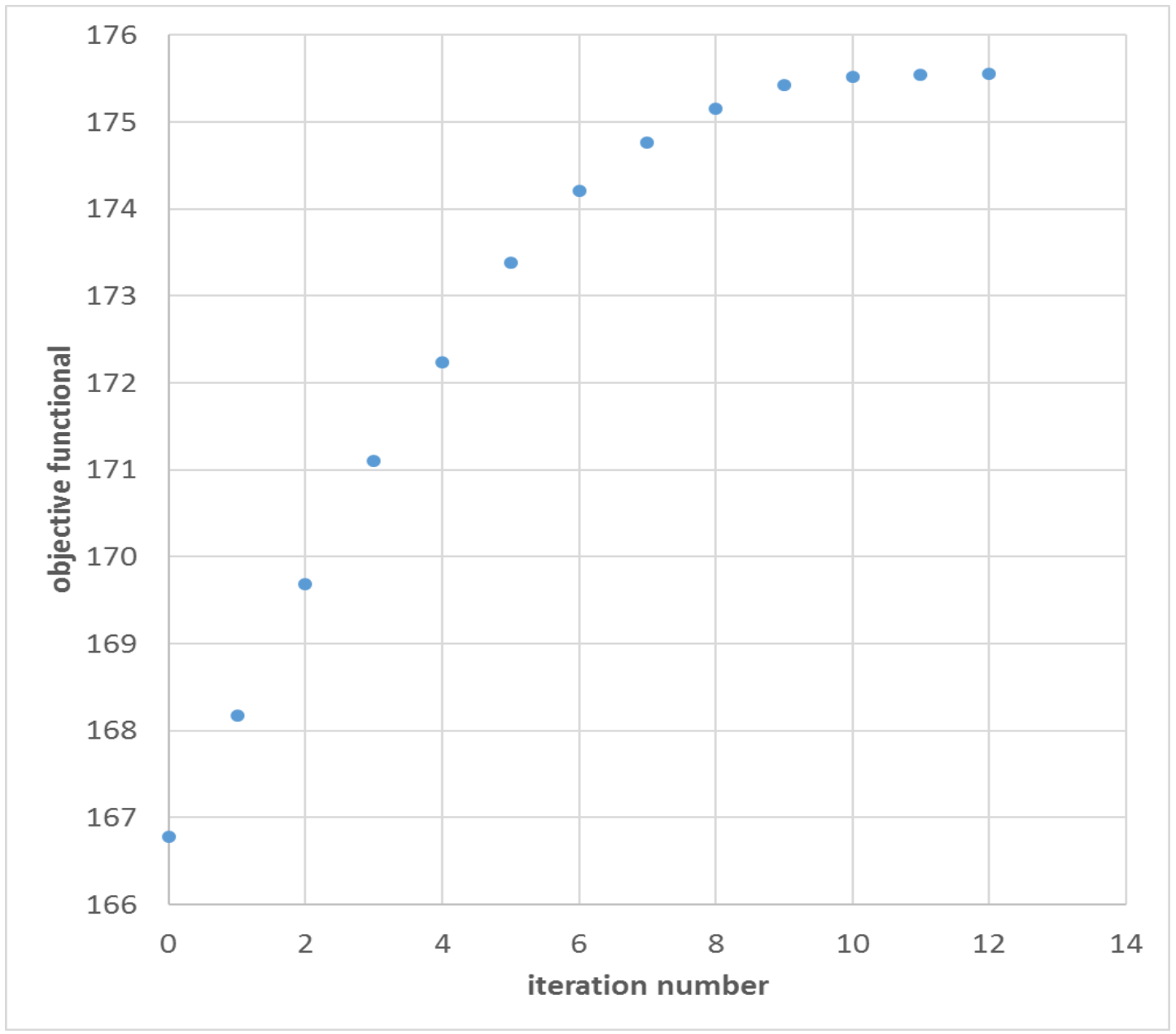

Figure 24: Objective functional versus iteration number for air injection (204 Darcy and Pressure $0.514 \mathrm{MPa})$

increased, the interfacial temperature started to reach maximum $90^{\circ} \mathrm{C}$.

Table 11 shows the final objective functional at five different initial interfacial temperatures. As it can be seen, by choosing the constant temperature of $25^{\circ} \mathrm{C}$ as an initial interfacial temperature guess, the maximum objective functional was 5.57 after 200 iterations. 
Table 11: Comparison of maximum objective functional obtained from different initial interfacial temperature

\begin{tabular}{|c|c|c|c|}
\hline $\begin{array}{l}\text { initial interfacial } \\
\text { temperature }\end{array}$ & iteration \# & final optimal function $\left[T_{\operatorname{int}}(t)\right]$ & $\begin{array}{l}\text { maximum } \\
\text { objective } \\
\text { functional }\end{array}$ \\
\hline $\begin{array}{l}\text { Periodic temperature } \\
\text { variation between } 90^{\circ} \mathrm{C} \\
\text { to } 75^{\circ} \mathrm{C} \\
\text { (after } 20 \mathrm{~min} \text { ) }\end{array}$ & 12 & $\begin{array}{l}\text { - first hour: constant } 90^{\circ} \mathrm{C} \\
\text { - afterward: periodic temperature } \\
\text { variation of } 90^{\circ} \mathrm{C} \text { to } 82^{\circ} \mathrm{C}\end{array}$ & 175.55 \\
\hline $\begin{array}{l}\text { Constant temperature of } \\
\qquad 90^{\circ} \mathrm{C} \\
\text { (after } 20 \mathrm{~min})\end{array}$ & 15 & $\begin{array}{l}\text { - first hour: constant } 90^{\circ} \mathrm{C} \\
\text { - afterward: periodic temperature } \\
\text { variation of } 90^{\circ} \mathrm{C} \text { to } 82^{\circ} \mathrm{C}\end{array}$ & 175.55 \\
\hline $\begin{array}{l}\text { Constant temperature of } \\
\qquad 25^{\circ} \mathrm{C}\end{array}$ & 200 & - constant $T_{\text {int }}$ of $32^{\circ} \mathrm{C}$ & 5.5700 \\
\hline $\begin{array}{l}\text { Periodic temperature } \\
\text { variation between } 90^{\circ} \mathrm{C} \\
\text { to } 50^{\circ} \mathrm{C} \\
\text { (after } 20 \mathrm{~min} \text { ) }\end{array}$ & 30 & $\begin{array}{l}\text { - first hour: constant } 90^{\circ} \mathrm{C} \\
\text { - afterward: periodic temperature } \\
\text { variation of } 90^{\circ} \mathrm{C} \text { to } 82^{\circ} \mathrm{C}\end{array}$ & 175.55 \\
\hline $\begin{array}{l}20 \text { minutes separation } \\
\text { between each periodic } \\
\text { variation of } 90^{\circ} \mathrm{C} \text { and } \\
\qquad 82^{\circ} \mathrm{C} \\
\text { (after } 20 \mathrm{~min} \text { ) }\end{array}$ & 13 & $\begin{array}{l}\text { - first hour: constant } 90^{\circ} \mathrm{C} \\
\text { - afterward: } 20 \text { min pulse periodic } \\
\text { temperature variation of } 90^{\circ} \mathrm{C} \text { to } \\
75^{\circ} \mathrm{C}\end{array}$ & 160.77 \\
\hline
\end{tabular}

Therefore, the optimal interfacial air temperature is to maintain $T_{\text {int }}$ at $90^{\circ} \mathrm{C}$ (after $20 \mathrm{~min}$ ) for 60 min, followed by periodic temperature variation between $90^{\circ} \mathrm{C}$ and $82^{\circ} \mathrm{C}$ with time (please see Figures 21 and 23). The maximum calculated objective functional is 175.55 . 


\subsubsection{Validation of Optimal Control Policy}

To validate the optimal injection temperature policy, the predicted objective functional corresponding to the optimal $\left[T_{\mathrm{int}}(t)\right]$ was compared to the experimental counterpart utilizing the same $\left[T_{\text {int }}(t)\right]$. In more detail, the simulated production rates were compared with the experimental ones obtained from another data set of an experiment conducted for the same physical model (set permeability) at the same operating conditions (optimal interfacial temperature $\left.\left[T_{\mathrm{int}}(t)\right]\right)$.

Figure 25 shows that the experimental and calculated heavy oil recovery agree very well for the same optimal $\left[T_{\text {int }}(t)\right]$. The results indicate that the predicted heavy oil recovery was $175.55 \mathrm{~g}$, and heavy oil recovered from the experimental work was $180.01 \mathrm{~g}$. Thus, the average of relative errors at all sample times in the mass of heavy oil recovered was $1.82 \%$. Furthermore, this figure shows the difference between the experimental optimal interfacial temperature and simulated interfacial temperature is very small with the average relative error less than $2 \%$. Therefore, the 


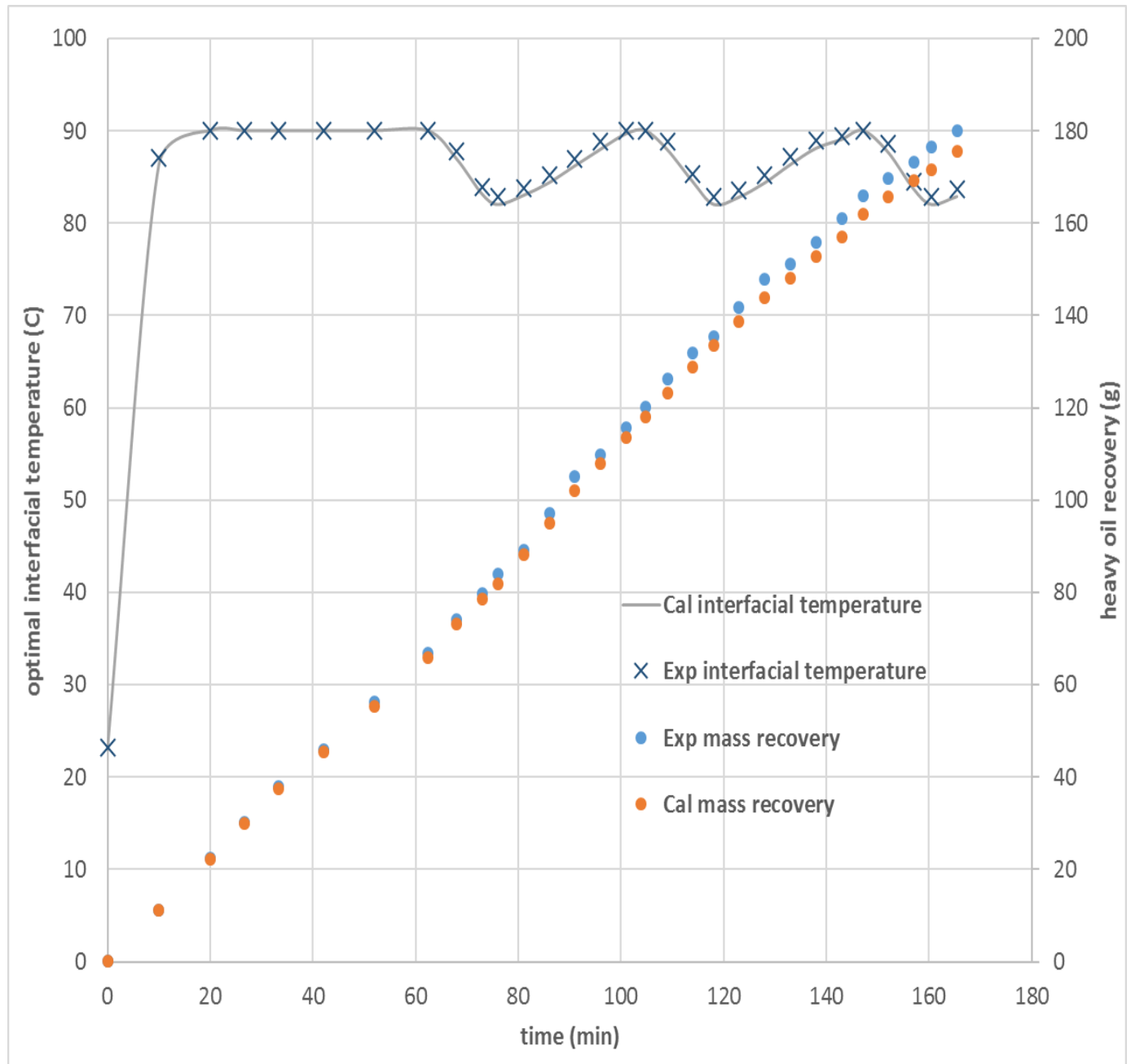

Figure 25: Experimental and calculated mass of live oil produced with time for air injection (Pressure 0.514 MPa, 204 Darcy)

small relative error demonstrates the confident of the optimal control strategy based on the developed process model.

\subsubsection{Enhancement of Heavy Oil Recovery}

The results in Table 11 shows that optimal $\left[T_{\text {int }}(t)\right]$ policy was achieved at periodic interfacial temperature variation between $90^{\circ} \mathrm{C}$ and $82^{\circ} \mathrm{C}$ (see Figures 21 and 22). The optimal $\left[T_{\text {int }}(t)\right]$ policy 
enhances the heavy oil recovery by $20.660 \%$ in comparison to that at the constant temperature of $90^{\circ} \mathrm{C}$.

\subsubsection{Permeability Effect on Optimal Policy}

In this section, we examine the impact of physical model permeability (40, 87, and 427 Darcy) on optimal $\left[T_{\text {int }}(t)\right]$ policy, as well as validation the model at different physical model permeability.

Figure 26 shows the air interfacial temperature versus time policy at the pressure of $0.514 \mathrm{MPa}$ and physical media permeability of 427 Darcy for 165 minutes of operational time. According to the figure, permeability has minor or no impact on optimal $\left[T_{\mathrm{int}}(t)\right]$. The objective functional improved upon increasing the permeability value to the maximum value of 260.19 (Figure 27).

The improvement of the objective functional at 427 Darcy (Figure 27) in comparison to computational run at 204 Darcy (Figure 22) may be attributed to the fact that the air can easier diffuse inside the physical model at a higher permeability. ${ }^{26}$ 


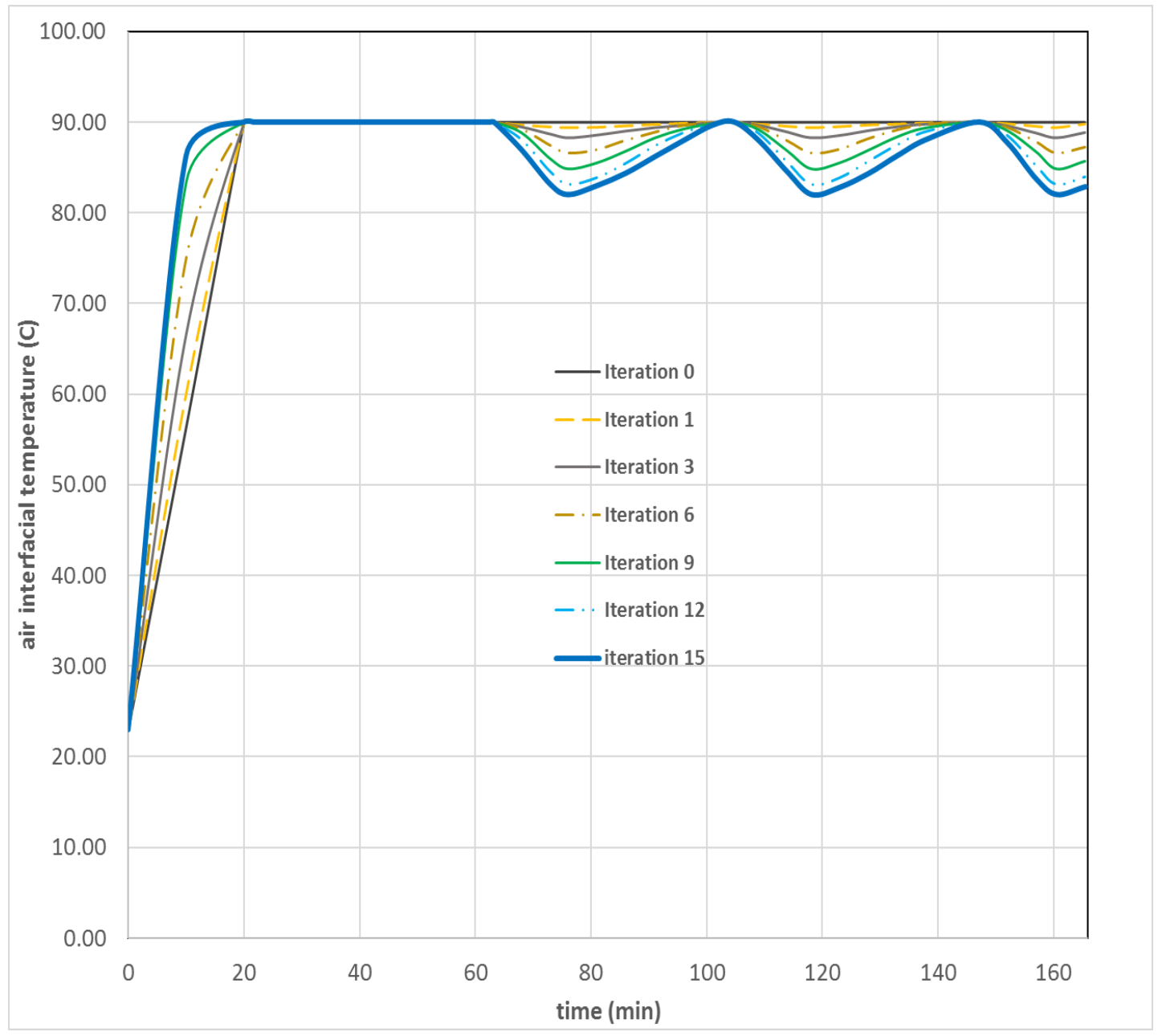

Figure 26: The interfacial air temperature $T_{\text {int }}(t)$ at physical model permeability of 427 Darcy and air pressure of $0.514 \mathrm{MPa}$ 


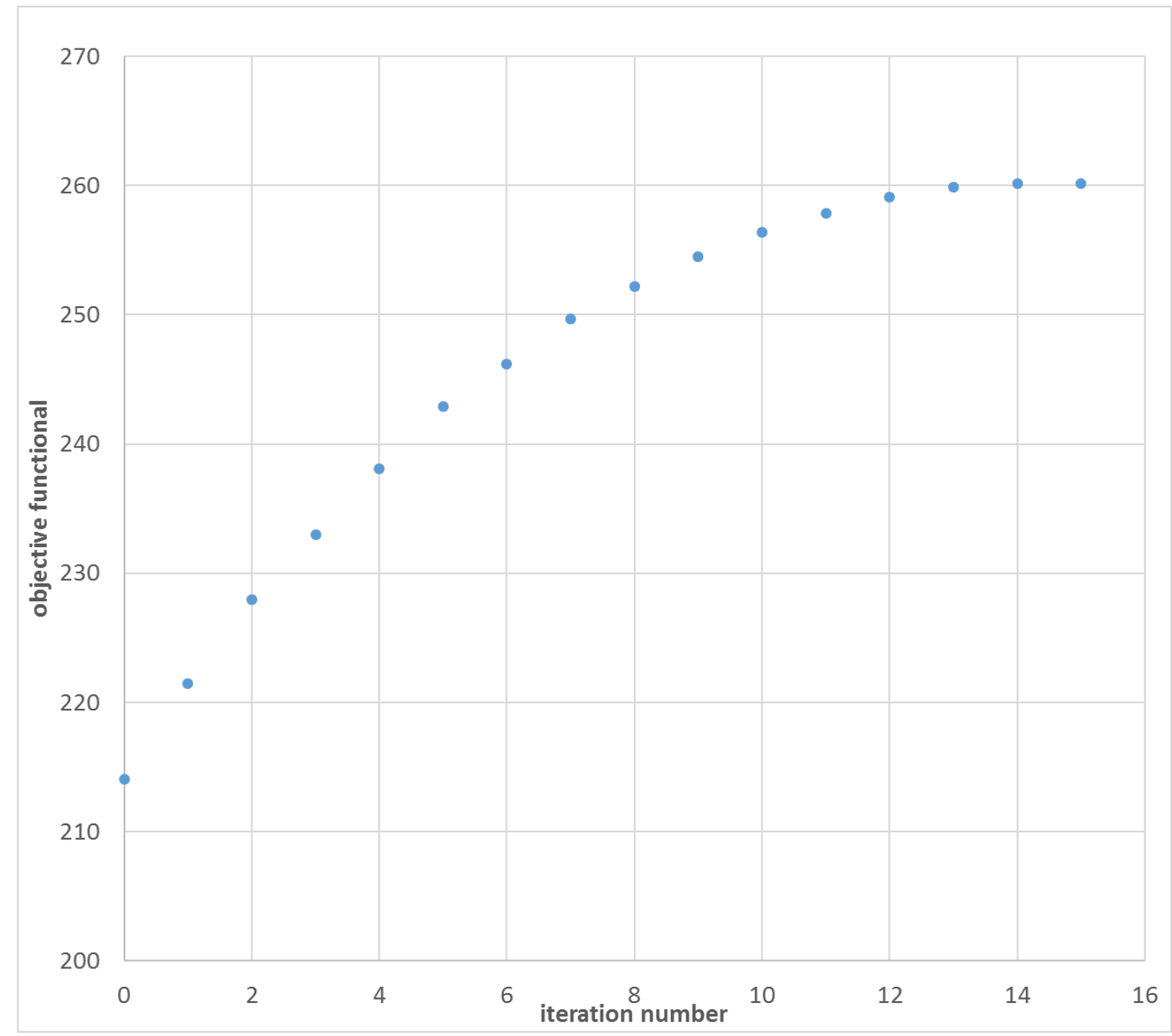

Figure 27: Objective functional versus iteration number for air injection (427 Darcy and Pressure $0.514 \mathrm{MPa}$ )

Figures 28 and 29 show the objective functional versus iteration number at 40 Darcy and 87 Darcy, respectively. Similar to Figure 24, the change was significant at the beginning, but the rate of improvement slowed down at final iteration. 


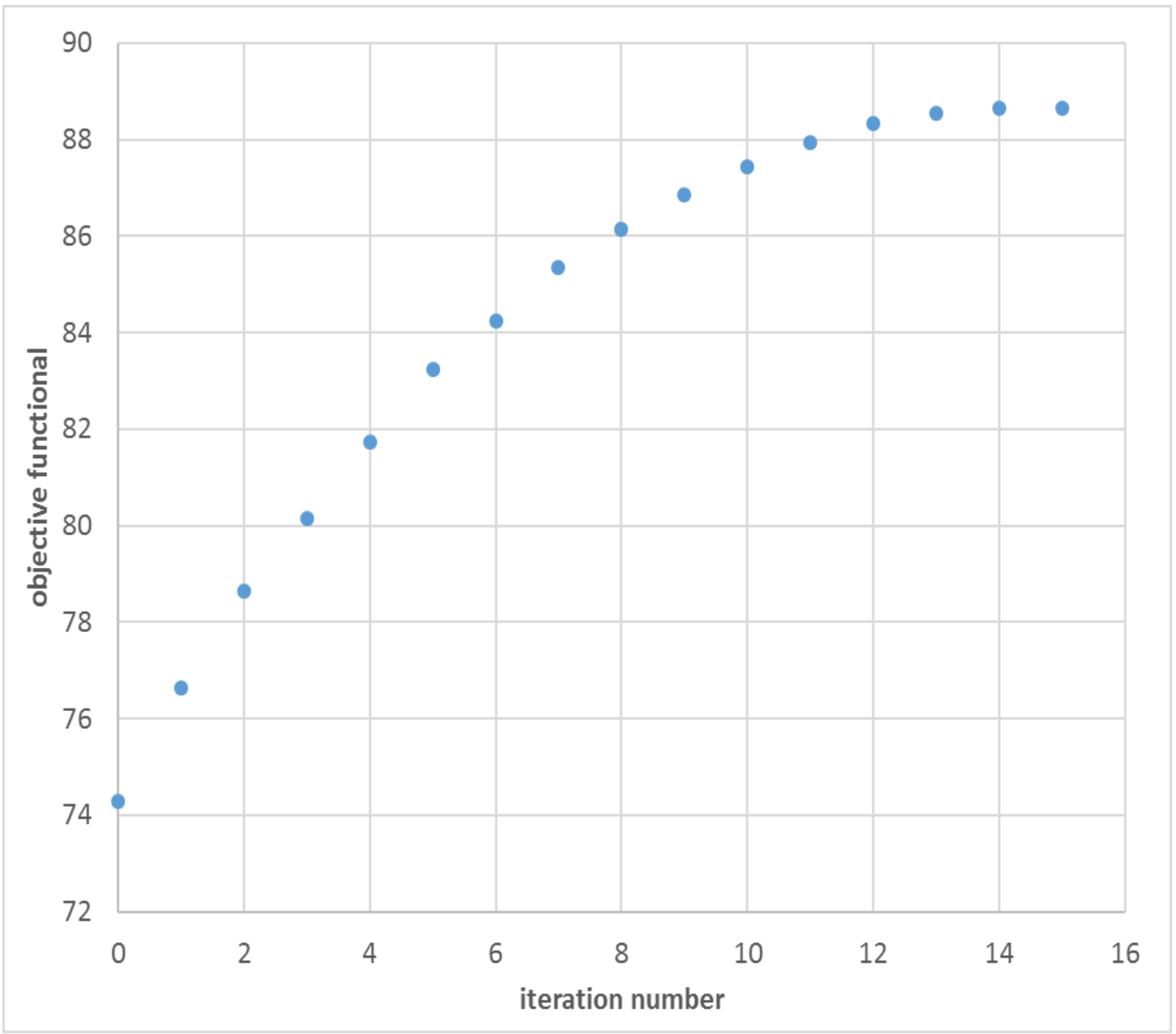

Figure 28: Objective functional versus iteration number for air injection (87 Darcy and Pressure $0.514 \mathrm{MPa}$ ) 


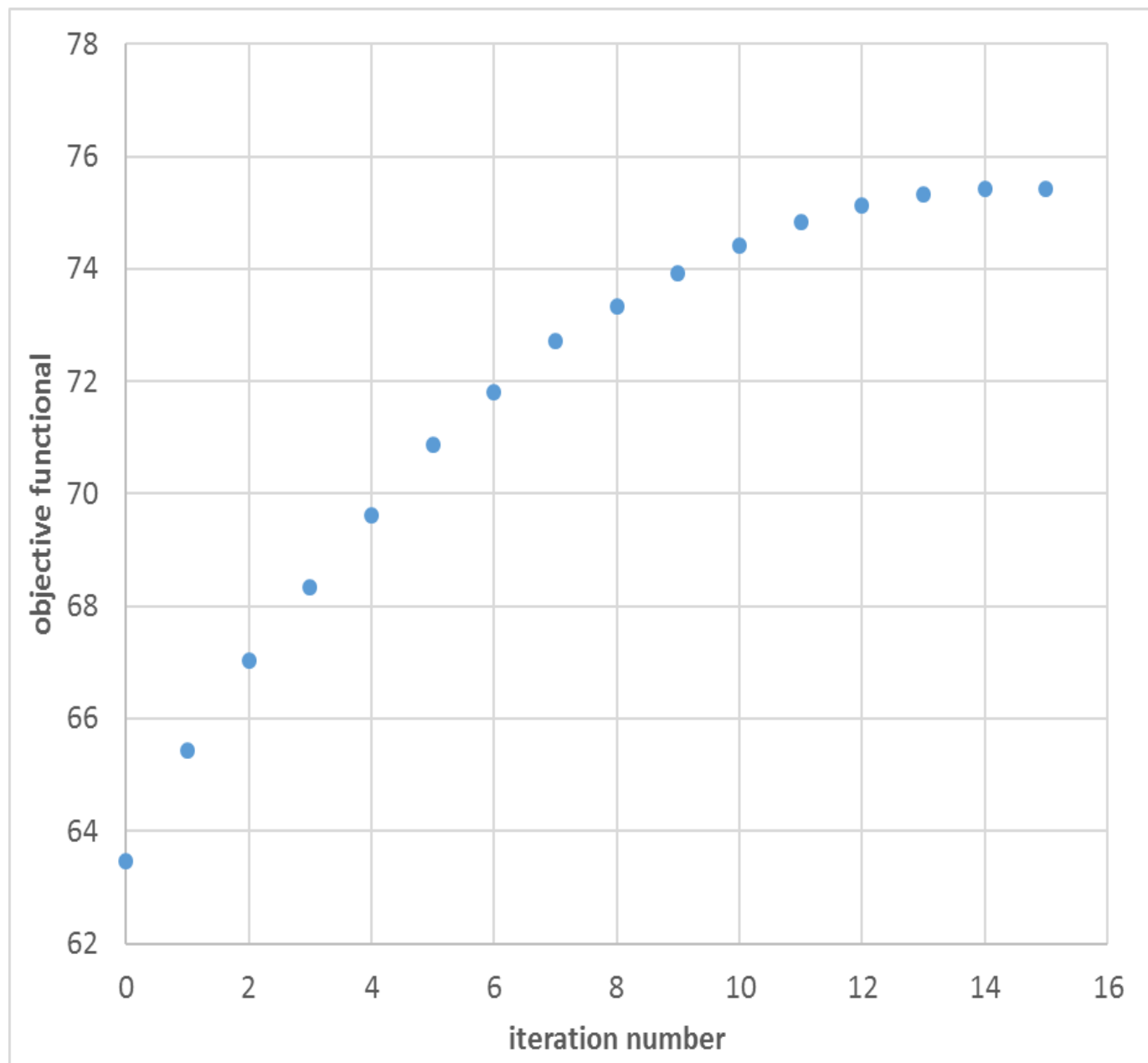

Figure 29: Objective functional versus iteration number for air injection (40 Darcy and Pressure $0.514 \mathrm{MPa})$

Lastly, it is required to validate the optimal control policy with experimental results for the computational runs above. Figure 30 shows the comparison between the calculated objective functional versus the experimental value at three different packs of permeability of 40,87 , and 427 Darcy. The average of relative errors for 40, 87, and 427 Darcy at all sample times in the mass of heavy oil recovered were found to be $2.1 \%, 2.8 \%$, and $3.6 \%$, respectively. Therefore, the low relative errors demonstrate that the optimal control developed in this study can be confidently applied to enhance oil recovery at different permeability as well. 


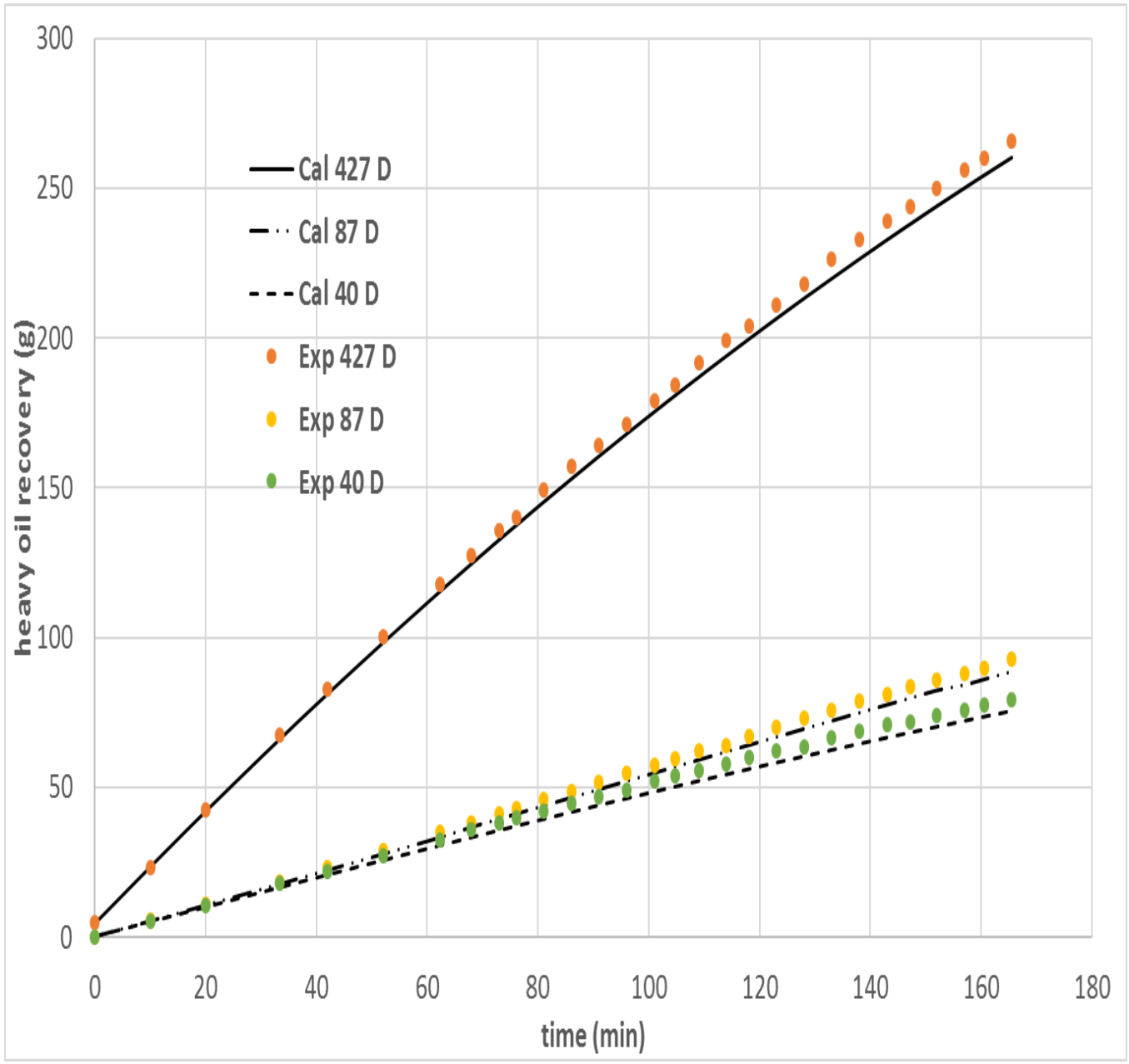

Figure 30: Experimental and calculated heavy oil recovery at three different medium permeability

\subsubsection{Sensitivity Analyses of the Model Parameters}

A sensitivity analysis of the model parameters on the calculated objective functional value was undertaken by varying live oil density $(\rho)$, live oil viscosity coefficient $(\mu)$, specific heat capacity $\left(C_{p}\right)$, thermal conductivity coefficient $(k)$, grids number, dispersion coefficient $[D(\omega, T)]$, and interfacial mass fraction $\left[\omega_{\text {int }}(t)\right]$.

The results of the sensitivity analyses were compared with the based final iteration objective functional value for 204 Darcy permeability, and 0.514 MPa (absolute) injection pressure. The 
sensitivity analyses were also experimentally validated. Table 12 shows the percentage change in the objective functional value.

- For $a \pm 5 \%$ variation in live oil density $(\rho)$, the change in the maximum objective functional is about $0.54 \%$ of the based value.

- For a $\pm 5 \%$ variation in viscosity coefficient $(\mu)$, the change in the maximum objective functional is about $1.16 \%$ of the based value.

- For a $\pm 5 \%$ variation in specific heat capacity $\left(C_{p}\right)$, the change in the maximum objective functional is about $0.55 \%$ of the based value

- For $\mathrm{a} \pm 5 \%$ variation in thermal conductivity coefficient $(k)$, the change in the maximum objective functional is about $0.48 \%$ of the based value

- The change in the maximum objective functional is about $1.3 \%$ of the based value when changing the number of grids in the radial direction. However, the change in maximum objective functional is about $0.7 \%$ of the based value when changing the number of grids in the vertical direction. The set modeling value for $\mathrm{N}_{\mathrm{z}}$ and $\mathrm{N}_{\mathrm{r}}$ are 20 and 10, respectively (Table 12).

- For a $\pm 5 \%$ variation in the dispersion coefficient $[D(\omega, T)]$, the change in the maximum objective functional is about $2.2 \%$ of the base value.

- For $\mathrm{a} \pm 5 \%$ variation in the air interfacial concentration $\left[\omega_{\text {int }}(t)\right]$ value, the change in the maximum objective functional is about $7.01 \%$ of the based value.

Thus, the objective functional is less sensitive to variation in live oil density, specific heat capacity, thermal conductivity coefficient, and grid numbers in comparison to viscosity coefficient $\left(\mu_{\mathrm{o}}\right)$, 
dispersion coefficient $[D(\omega, T)]$, and interfacial solvent concentration $\left[\omega_{\text {int }}(t)\right]$. Also, the initial $\left[T_{\mathrm{int}}(t)\right]$ guess is very important to determine the maximum objective functional (Table 11).

Lastly, we examined the sensitivity of initial dispersion $[D(\omega, T)]$ compared to optimal dispersion coefficient for 204 Darcy permeability, and $0.514 \mathrm{MPa}$ (absolute) injection pressure. For a $\pm 5 \%$ variation in the initial dispersion coefficient, the change in dispersion value is about $0.2 \%$ of the base value.

Table 12: Change in maximum objective functional by variation in parameters

\begin{tabular}{|c|c|c|}
\hline parameter & variation in parameter & $\%$ change in maximum I \\
\hline \multirow[t]{2}{*}{ live oil density } & $+5 \%$ & -0.54 \\
\hline & $-5 \%$ & 0.54 \\
\hline \multirow[t]{2}{*}{ viscosity coefficient } & $+5 \%$ & 1.16 \\
\hline & $-5 \%$ & -1.16 \\
\hline \multirow[t]{2}{*}{ specific heat capacity } & $+5 \%$ & -0.55 \\
\hline & $-5 \%$ & 0.55 \\
\hline thermal conductivity & $+5 \%$ & -0.48 \\
\hline coefficient & $-5 \%$ & 0.48 \\
\hline \multirow[t]{4}{*}{ grid numbers } & $\mathrm{N}_{\mathrm{r}}=25, \mathrm{~N}_{\mathrm{z}}=10$ & -1.29 \\
\hline & $\mathrm{N}_{\mathrm{r}}=15, \mathrm{~N}_{\mathrm{z}}=10$ & 1.29 \\
\hline & $\mathrm{N}_{\mathrm{r}}=20, \mathrm{~N}_{\mathrm{z}}=12$ & -0.70 \\
\hline & $\mathrm{N}_{\mathrm{r}}=20, \mathrm{~N}_{\mathrm{Z}}=7$ & 0.70 \\
\hline \multirow[t]{2}{*}{ dispersion coefficient } & $+5 \%$ & -2.20 \\
\hline & $-5 \%$ & 2.20 \\
\hline \multirow[t]{2}{*}{ interfacial concentration } & $+5 \%$ & -7.01 \\
\hline & $-5 \%$ & 7.01 \\
\hline
\end{tabular}




\subsubsection{Potential for Field Scale}

In field scale heavy oil reservoirs that are more than a few kilometers thick, the temperature and pressure would exceed the limits of this study. There is a possibility of minor oxidation reactions upon air injection into such reservoirs for more than a year. ${ }^{58,59}$

Given this caveat, the results of this experimental study are pertinent to heavy oil recovery from shallow reservoirs at low temperature and pressure conditions that are conducive to non-reactive environments. For those circumstances, this study demonstrates a concept that air injection with periodic temperature variation has the potential to enable significant oil recovery.

Table 13 shows heavy oil recoveries reported in previous investigations, which used different gas solvents with physical models similar to the ones in this study. It is observed that the recovery of 69.1\% OOIP obtained with air in this study is, on an average, about $16 \%$ lower compared to that with propane or butane. ${ }^{49,50,61}$

This is very encouraging given the fact that air is freely available while hydrocarbon solvents are costly and their use for oil recovery is therefore almost impractical at present. ${ }^{22,57}$ 
Table 13: Oil recoveries obtained in previous studies with different solvents

\begin{tabular}{|c|c|c|c|c|c|c|}
\hline reservoir model & solvent & heavy oil & $T\left({ }^{\circ} \mathrm{C}\right)$ & $\boldsymbol{P}(\mathrm{MPa})$ & $\%$ OOIP & ref. no. \\
\hline $\begin{array}{l}\text { cylindrical sand packed } \\
\text { model with glass beads. } \\
\text { Physical model } \\
\text { permeability: } 44.4,97.4, \\
\text { 220, and 439.2 Darcy }\end{array}$ & butane & $\begin{array}{c}\text { Fort Kent } \\
(14,500 \mathrm{mPa} \cdot \mathrm{s})\end{array}$ & 21 & $\begin{array}{l}0.09- \\
0.112\end{array}$ & 80 & 49 \\
\hline $\begin{array}{c}\text { cylindrical sand packed } \\
\text { model with glass beads. } \\
\text { Physical model } \\
\text { permeability: } 44.4,97.4 \text {, } \\
\text { 220, and 439.2 Darcy }\end{array}$ & propane & $\begin{array}{c}\text { Fort Kent } \\
(14,500 \mathrm{mPa} \cdot \mathrm{s})\end{array}$ & 21 & $\begin{array}{c}0.413- \\
0.68\end{array}$ & 92 & 50 \\
\hline $\begin{array}{l}\text { cylindrical sand packed } \\
\text { model with glass beads. } \\
\text { Physical model } \\
\text { permeability: 51, } 102, \\
\text { and } 204 \text { Darcy }\end{array}$ & propane & $\begin{array}{c}\text { Athabasca } \\
(225,000 \mathrm{MPa} . \mathrm{s})\end{array}$ & 21 & 0.790 & 89 & 48 \\
\hline $\begin{array}{l}\text { cylindrical sand packed } \\
\text { model with glass beads. } \\
\text { Physical model } \\
\text { permeability: } 110 \text { Darcy }\end{array}$ & butane & $\begin{array}{c}\text { Fort Kent } \\
(14,500 \mathrm{mPa} \cdot \mathrm{s})\end{array}$ & 23 & 0.11 & 85 & 57 \\
\hline
\end{tabular}




\section{Chapter 6: Conclusions}

In this work, air injection was considered for the first time to recover heavy oil at low temperature and pressure conditions under a non-reactive environment in a solvent-assisted gravity drainage process. The effect of constant as well as periodic air temperatures was examined in lab-scale experiments. The air was injected at $0.169,0.286,0.403$ and $0.514 \mathrm{MPa}$ absolute and temperatures in the range $25^{\circ} \mathrm{C}-90^{\circ} \mathrm{C}$ into lab-scale physical models of $40,87,204$, and 427 Darcy permeability and $38 \%$ porosity. The maximum heavy oil recovery of $58.2 \%$ of the OOIP was achieved using air at the constant temperature of $90^{\circ} \mathrm{C}$ and $0.514 \mathrm{MPa}$ pressure with 427 Darcy permeability of the physical model. The periodic variation of the air temperature between $75^{\circ} \mathrm{C}$ and $90^{\circ} \mathrm{C}$ boosted that recovery to $69.1 \%$ of the OOIP, which is an improvement by $18.6 \%$. Overall, the results showed that the utilization of freely available air at low pressures and periodically varying low temperatures was promising for oil recovery from shallow reservoirs under non-reactive conditions.

In the next step, a rigorous mathematical model of the lab-scale recovery process was developed with interfacial air temperature versus time as a control function. The conditions necessary for optimal control were derived and utilized in a computational algorithm to determine the optimal control.

Following are the salient contributions stemming from the mathematical and computational part of this work: 
1. The optimal interfacial temperature versus time (control policy) was determined between $90^{\circ} \mathrm{C}$ and $82^{\circ} \mathrm{C}$, which registered $20.66 \%$ increase in the oil recovery in comparison to the constant temperature of $90^{\circ} \mathrm{C}$.

2. The control policy was successfully validated using experiments. The heavy oil recovery with the optimal $\left[T_{\mathrm{int}}(t)\right]$ was found to be within $1.82 \%$ of that predicted by the optimal control algorithm.

3. The computation time reduced from 2 weeks to 5 days by scaling the dependent variables of the differential equations.

The recommendations for future work are as follows:

1. Optimal control algorithm used in this study is open loop. Investigate the performance of a close-loop optimal control for the system.

2. Improve the heavy oil experimental setup to enable quicker heating and cooling of the system, and explore better temperature policies.

3. Carry out similar investigations using different gases (i.e., carbon dioxide, propane, and butane) to gain deeper understanding of the effect of solvent temperature oscillations on oil production. 


\section{Appendix A: Porous Medium Permeability Calculation}

Sample calculation of porous media of 40 Darcy:

The permeability (K) was calculated by applying the following equation (Dullien, 1992)

$K=\frac{Q \mu \Delta x}{A \Delta P} \times 1.01325 \times 10^{12}$

where $Q$ is the volumetric fluid flow rate through the media, $A$ is the cross-section area of fluid flow through the medium, $\mu$ is the dynamic viscosity of the fluid, and $\Delta P$ is the pressure difference across the medium of thickness $\Delta x$. The values of these parameters are listed in Table 4 for glass beads of size in the range $0.248-0.210 \mathrm{~mm}$.

$$
\begin{gathered}
K=\frac{\left(1.66001 \times 10^{-5}\right)\left(1.8401 \times 10^{-5}\right)(0.21)}{\left(2.82 \times 10^{-3}\right)(577)} \times 1.01325 \times 10^{12} \\
K=40 \text { Darcy }
\end{gathered}
$$




\section{Appendix B: Solubility and Live Oil Density}

Sample calculation of air solubility (or dissolved mass fraction) and live oil density.

To calculate the atmospheric air solubility and live oil density, substitute in Equations (13) and (14) for all the parameters from Table 14:

Air dissolved mass fraction $=\frac{0.15302}{9.6421+0.15302}=0.015620$

Live oil density $=\frac{9.6421+0.15302}{11.731}=0.83481$

Table 14: Calculated air solubility and live oil density at $25^{\circ} \mathrm{C}$ and 204 Darcy

\begin{tabular}{cccccc}
$\begin{array}{c}\text { pressure } \\
(\mathrm{MPa} \\
\text { absolute })\end{array}$ & $\begin{array}{c}\text { dead oil } \\
\text { mass }(\mathrm{g})\end{array}$ & $\begin{array}{c}\text { live oil } \\
\text { volume }\left(\mathbf{c m}^{\mathbf{3}}\right)\end{array}$ & $\begin{array}{c}\text { collected air } \\
\text { mass }(\mathrm{g})\end{array}$ & $\begin{array}{c}\text { live oil } \\
\text { density } \\
\left(\mathbf{g} / \mathbf{c m}^{\mathbf{3}}\right)\end{array}$ & $\begin{array}{c}\text { dissolved air } \\
\text { mass fraction }\end{array}$ \\
\hline 0.169 & 4.6710 & 5.8105 & 0.035010 & 0.80941 & 0.0074370 \\
\hline 0.286 & 6.2632 & 7.7601 & 0.064030 & 0.81482 & 0.010115 \\
\hline 0.403 & 8.0610 & 9.9411 & 0.10601 & 0.82144 & 0.012979 \\
\hline 0.514 & 9.6402 & 11.731 & 0.15302 & 0.83481 & 0.015620
\end{tabular}

Also, Table 15 shows the air solubility, live oil density, and viscosity at different temperatures.

Table 15: The result of live oil viscosity, live oil density, and air solubility at different temperatures

\begin{tabular}{cccc}
$\begin{array}{c}\text { temperature } \\
\left({ }^{\circ} \mathrm{C}\right)\end{array}$ & $\begin{array}{c}\text { viscosity } \\
(\mathrm{mPa} . \mathrm{s})\end{array}$ & $\begin{array}{c}\text { live oil } \\
\text { density } \\
\left(\mathbf{g} / \mathbf{c m}^{\mathbf{3}}\right)\end{array}$ & $\begin{array}{c}\text { dissolved } \\
\text { air mass } \\
\text { fraction }\end{array}$ \\
\hline 25 & 11,500 & 0.83481 & 0.015601 \\
\hline 50 & 642.00 & 0.81510 & 0.061102 \\
\hline 75 & 86.800 & 0.79582 & 0.16603 \\
\hline 90 & 11.200 & 0.78452 & 0.46301
\end{tabular}


Lastly, Figure 31 shows the correlation between air temperature and viscosity in this study.

$\mu=2 \times 10^{11}(T)^{-5.118}$

with the $r^{2}$ value of 0.9671 .

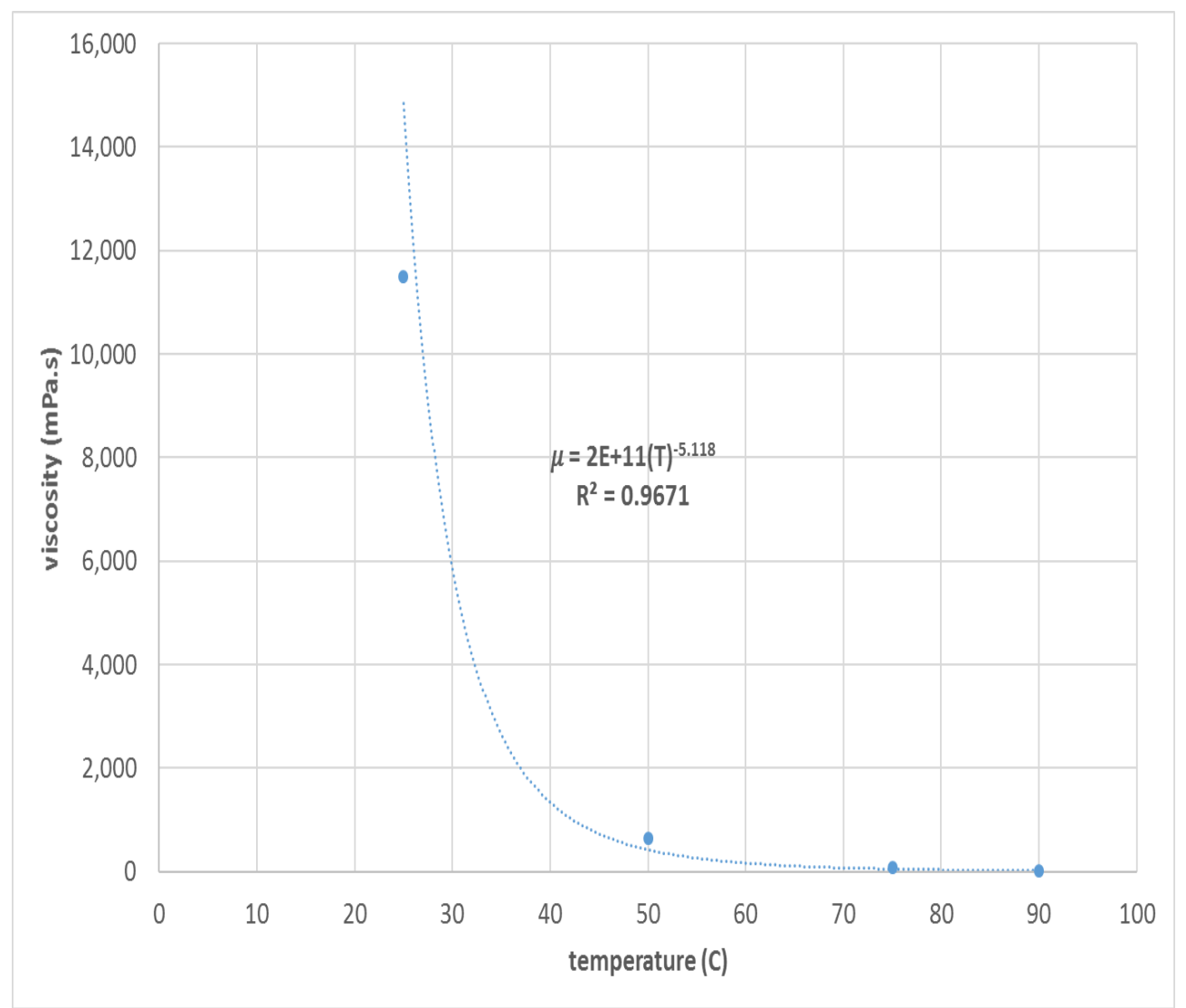

Figure 31: Variation of viscosity with air temperature at $0.514 \mathrm{MPa}$ absolute and 204 Darcy permeability of the physical model 


\section{Appendix C: Preliminary Experimental Results for Nitrogen Injection}

This appendix presents the impacts of permeability, pressure, and temperature on heavy oil recovery using nitrogen gas. In all the preliminary experiments, the experimental conditions are exactly same as atmospheric air injection, except in this part we have used pure nitrogen as a solvent (purity 99.9\%)

\section{$\underline{\text { Impact of Permeability }}$}

According to Figure 32, the heavy oil recovery increased marginally higher than atmospheric air injection ( 0.71 to $2.36 \%$ of the OOIP) (Figure 7$)$. This may be because unlike injection pure nitrogen gas, the atmospheric air injected has about $78 \%$ nitrogen gas.

Lastly, Figure 33 shows the correlation between the production rate and permeability. In both cases (air and nitrogen), the correlation has the same pattern. 


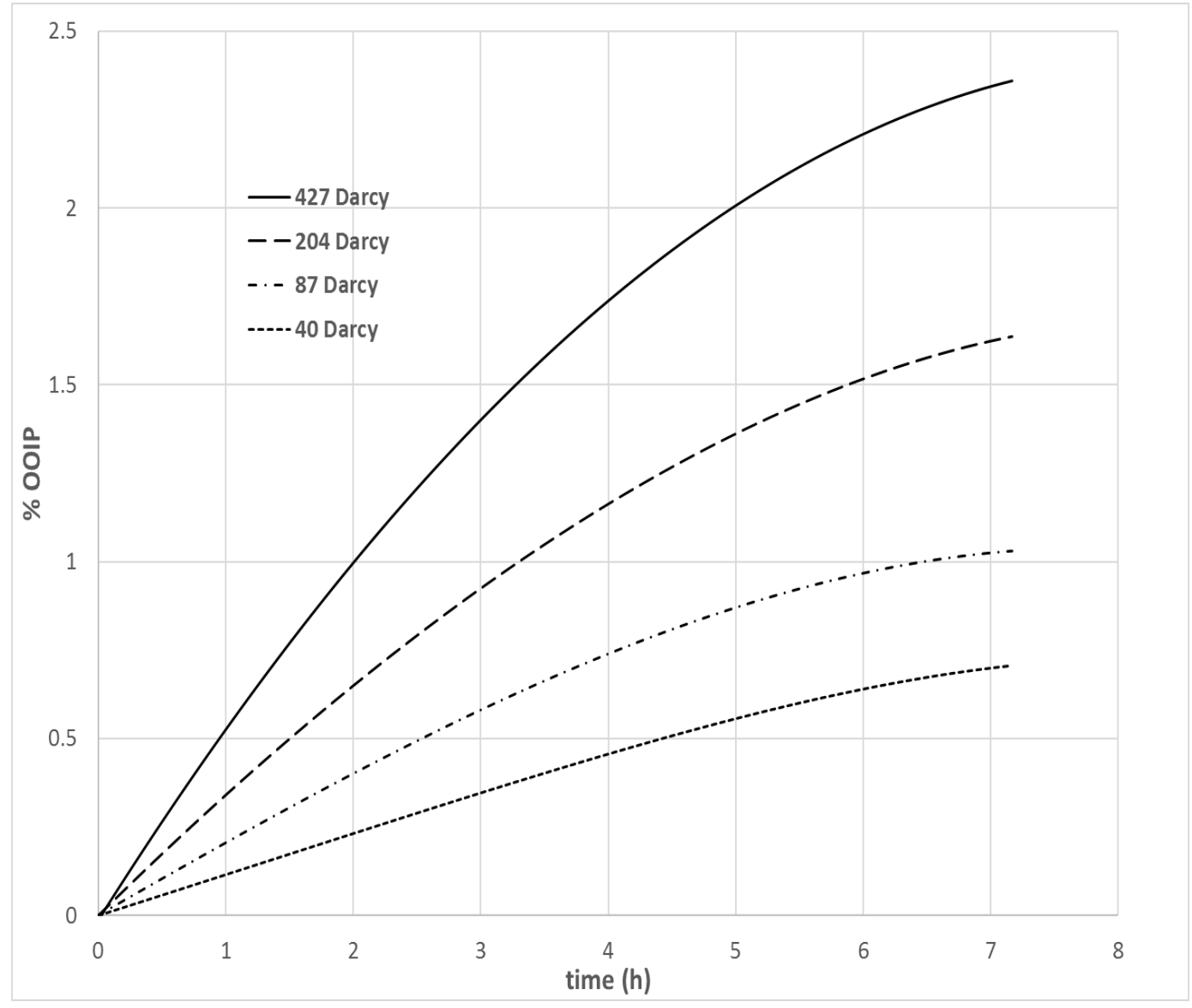

Figure 32: Oil recovery versus time for different permeabilities at $0.514 \mathrm{MPa}$ absolute and $25^{\circ} \mathrm{C}$. (Solvent: Nitrogen) 


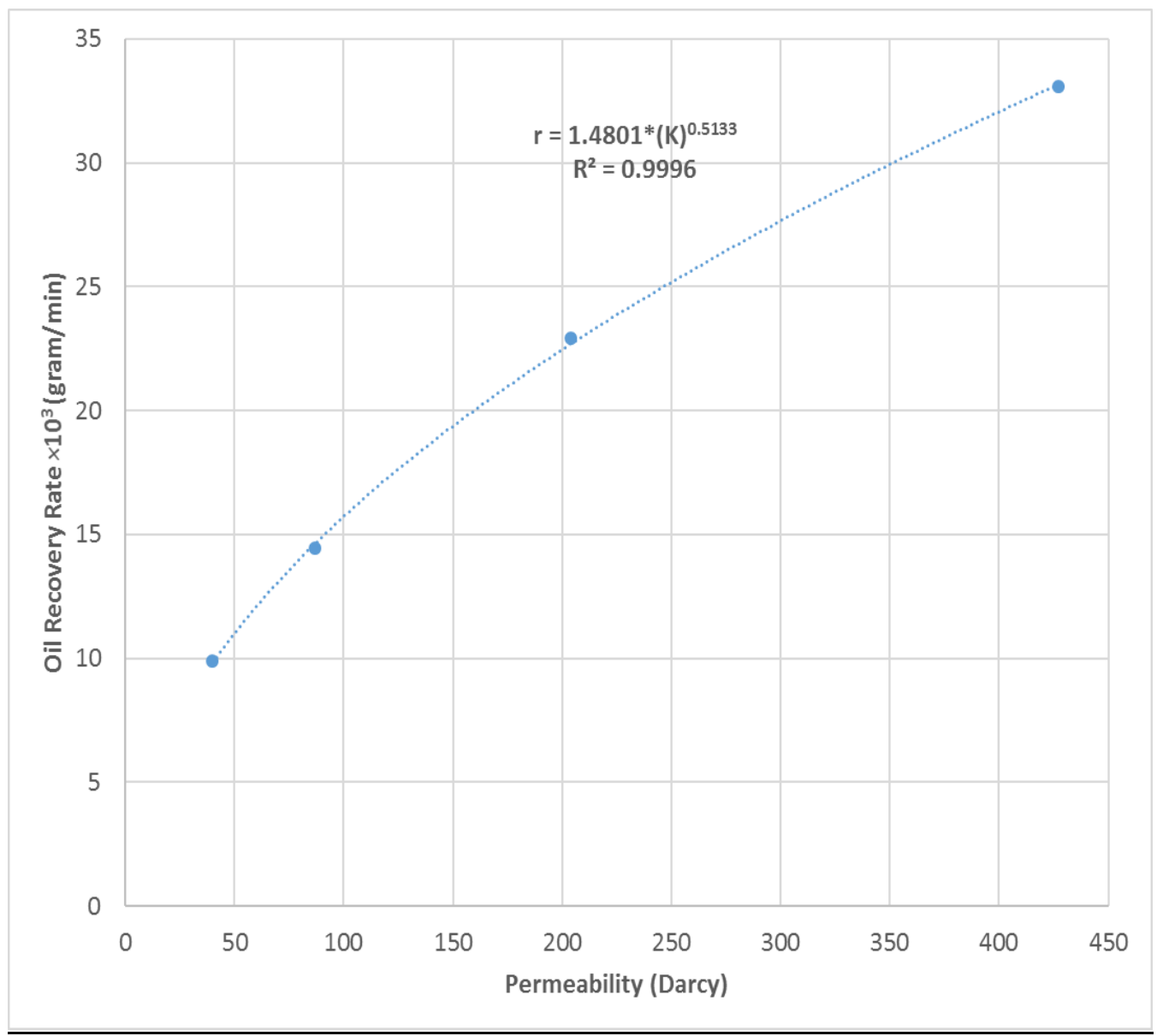

Figure 33: Variation of production rate with model permeability at pressure of $0.514 \mathrm{MPa}$ absolute and temperature of $25^{\circ} \mathrm{C}$ (Solvent: Nitrogen) 


\section{$\underline{\text { Impact of Pressure }}$}

As it can be seen in Figure 34, as the injection nitrogen pressure increased, the recovery factor using nitrogen gas increased from $0.78 \%$ to $1.65 \%$ of the OOIP.

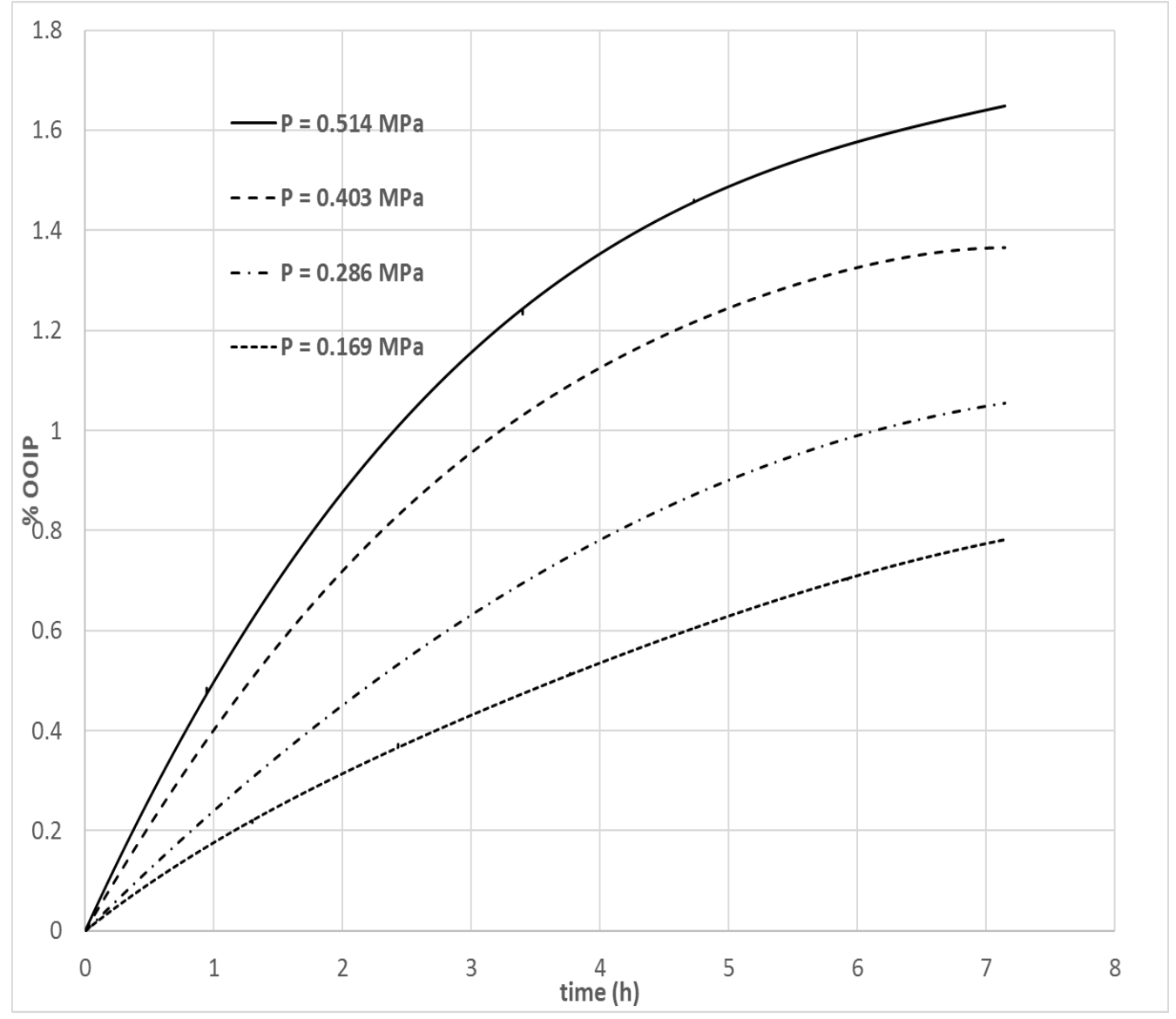

Figure 34: Oil recovery versus time at $25^{\circ} \mathrm{C}$ nitrogen temperature, 204 Darcy model permeability, and different air pressures (absolute) 


\section{Impact of Constant Temperature and Periodic Temperature Variation}

Similar to atmospheric air injection, the diffusivity of nitrogen in heavy oil was found to increase with temperature (Figure 35). The results show that by increasing temperature from $25^{\circ} \mathrm{C}$ to $90^{\circ} \mathrm{C}$, oil recovery rose from $1.63 \%$ to $42.41 \%$ of the OOIP.

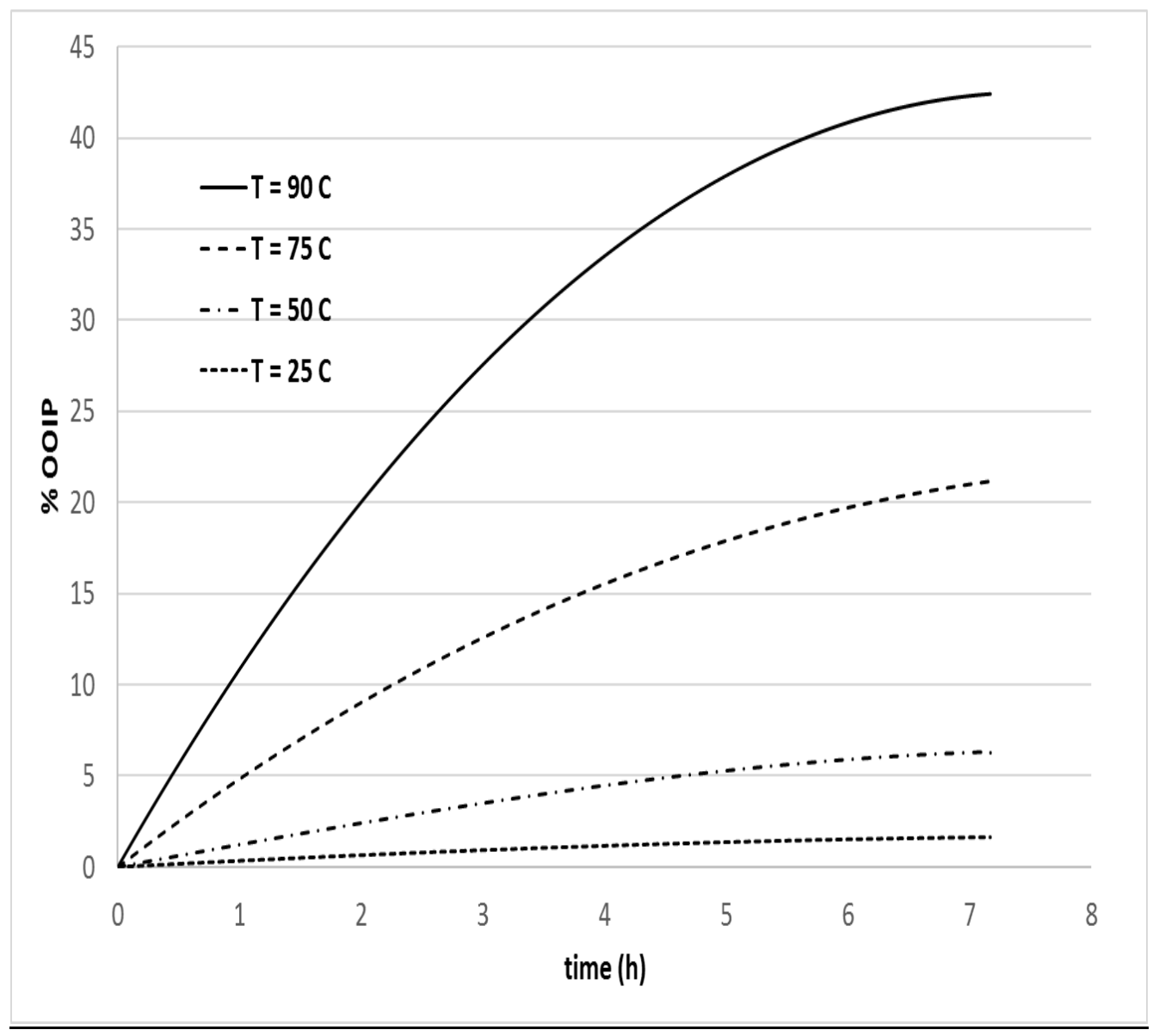

Figure 35: Oil recovery versus time at $0.514 \mathrm{MPa}$ absolute nitrogen pressure, 204 Darcy model permeability, and different nitrogen temperatures

We have also compared the effect of periodic temperature variation using nitrogen for heavy oil recovery under same experimental conditions (see Section 5.1.3). Figure 36 shows the impact of periodic temperature variation between $90^{\circ} \mathrm{C}$ and $75^{\circ} \mathrm{C}$ at $0.414 \mathrm{MPa}$ and the physical model of 
204 Darcy. As it was expected, we observed an enhancement of oil recovery increased from $42.41 \%$ to $49.86 \%$ of the OOIP using period temperature variation in comparison to the constant temperature $\left(90^{\circ} \mathrm{C}\right)$.

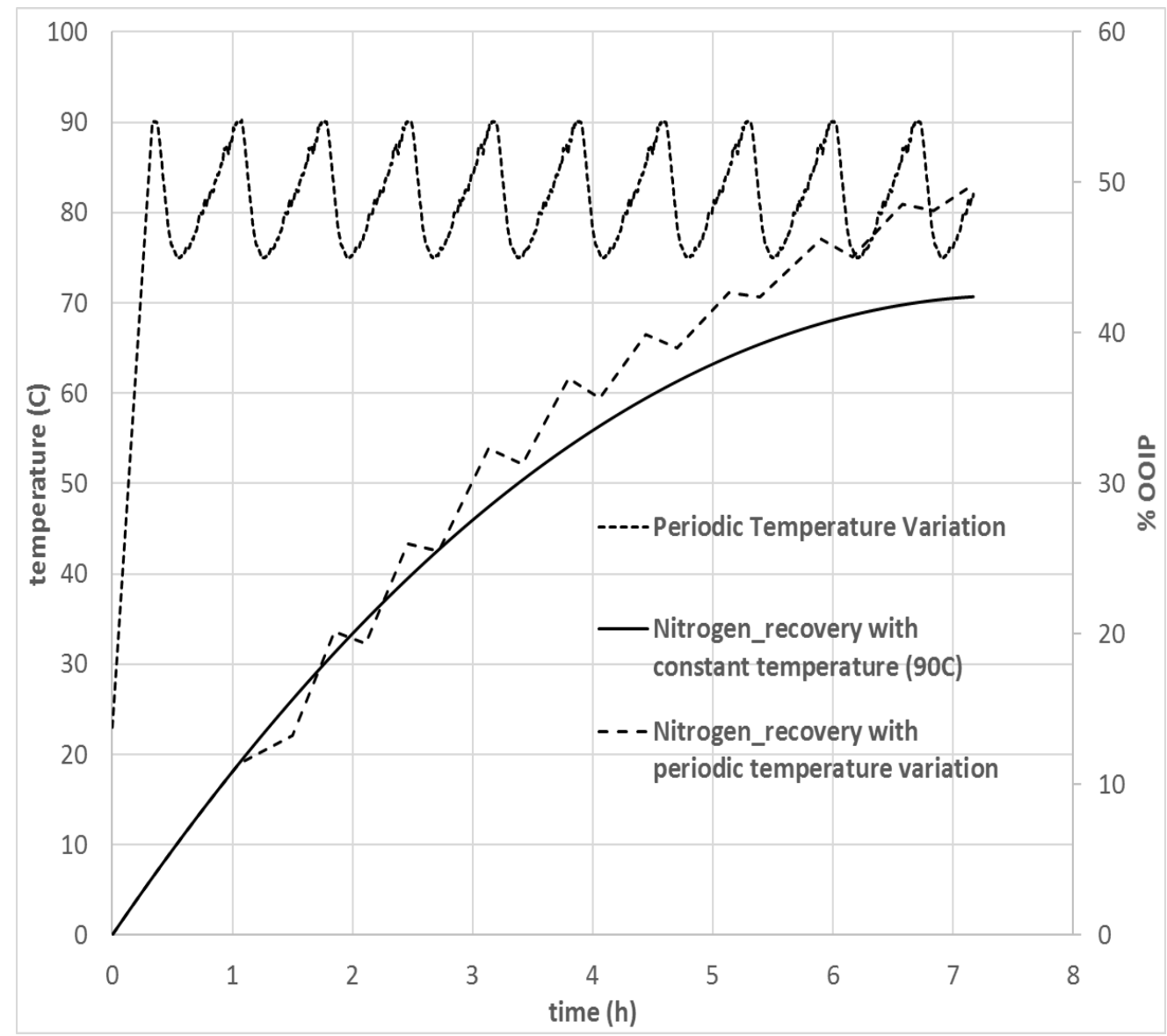

Figure 36: Oil recovery versus time at $0.514 \mathrm{MPa}$ absolute Nitrogen pressure, 204 Darcy model permeability, and constant $\left(90^{\circ} \mathrm{C}\right)$ as well as periodically varying nitrogen temperature in the range, $75-90^{\circ} \mathrm{C}$ 


\section{Effect of Varying Temperature with Different Permeabilities for Nitrogen Injection}

Similar results were observed using nitrogen injection under period temperature variation between $90^{\circ} \mathrm{C}$ and $75^{\circ} \mathrm{C}$, and different physical model permeability with the highest increase in \%OOIP was achieved as 427 Darcy with the value of $\% 71.20$ (Figures 37-39).

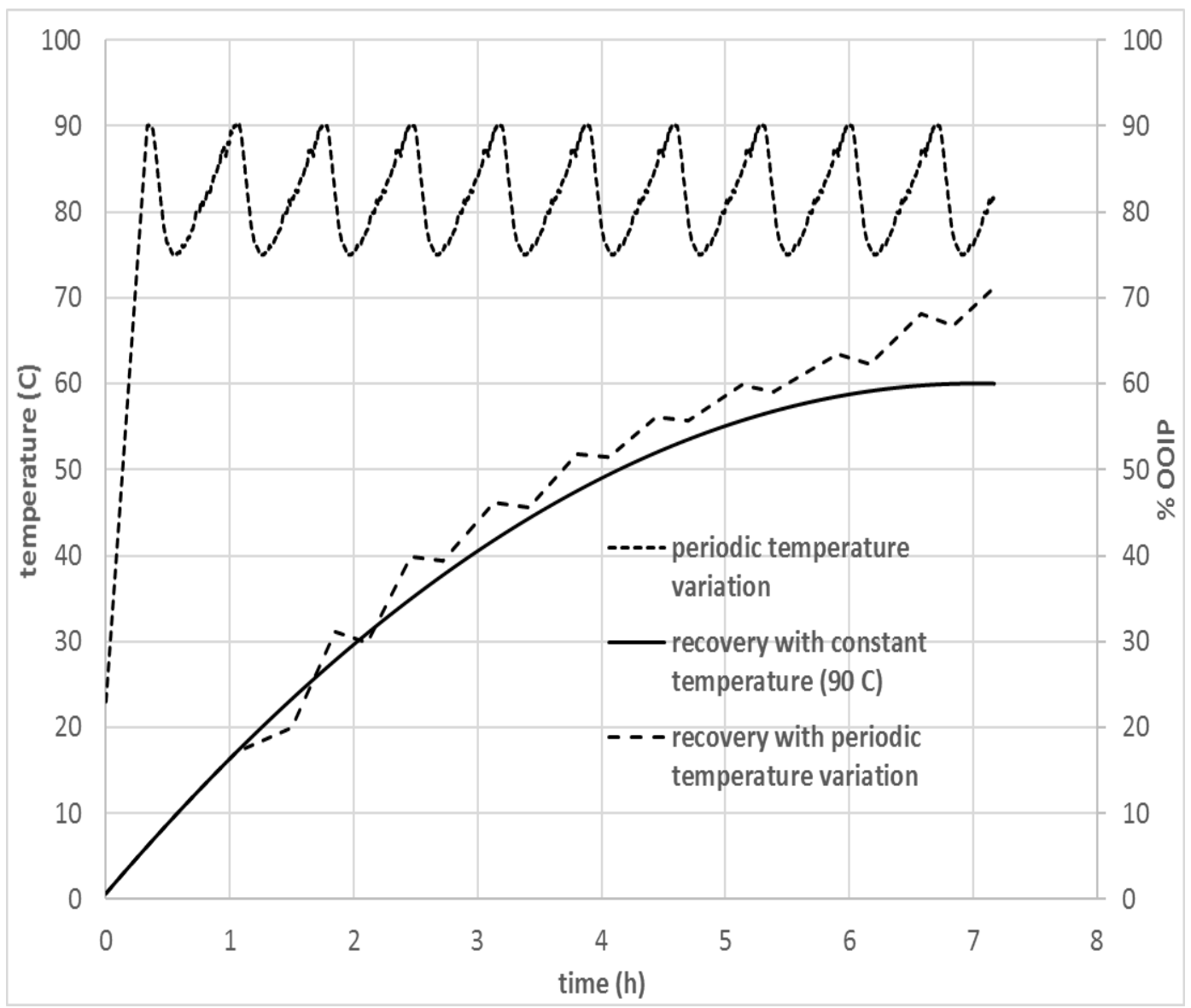

Figure 37: Oil recovery versus time at $0.514 \mathrm{MPa}$ absolute nitrogen pressure, constant $\left(90^{\circ} \mathrm{C}\right)$ as well as periodically varying air temperature in the range, $75-90^{\circ} \mathrm{C}$, and 427 Darcy model permeability 


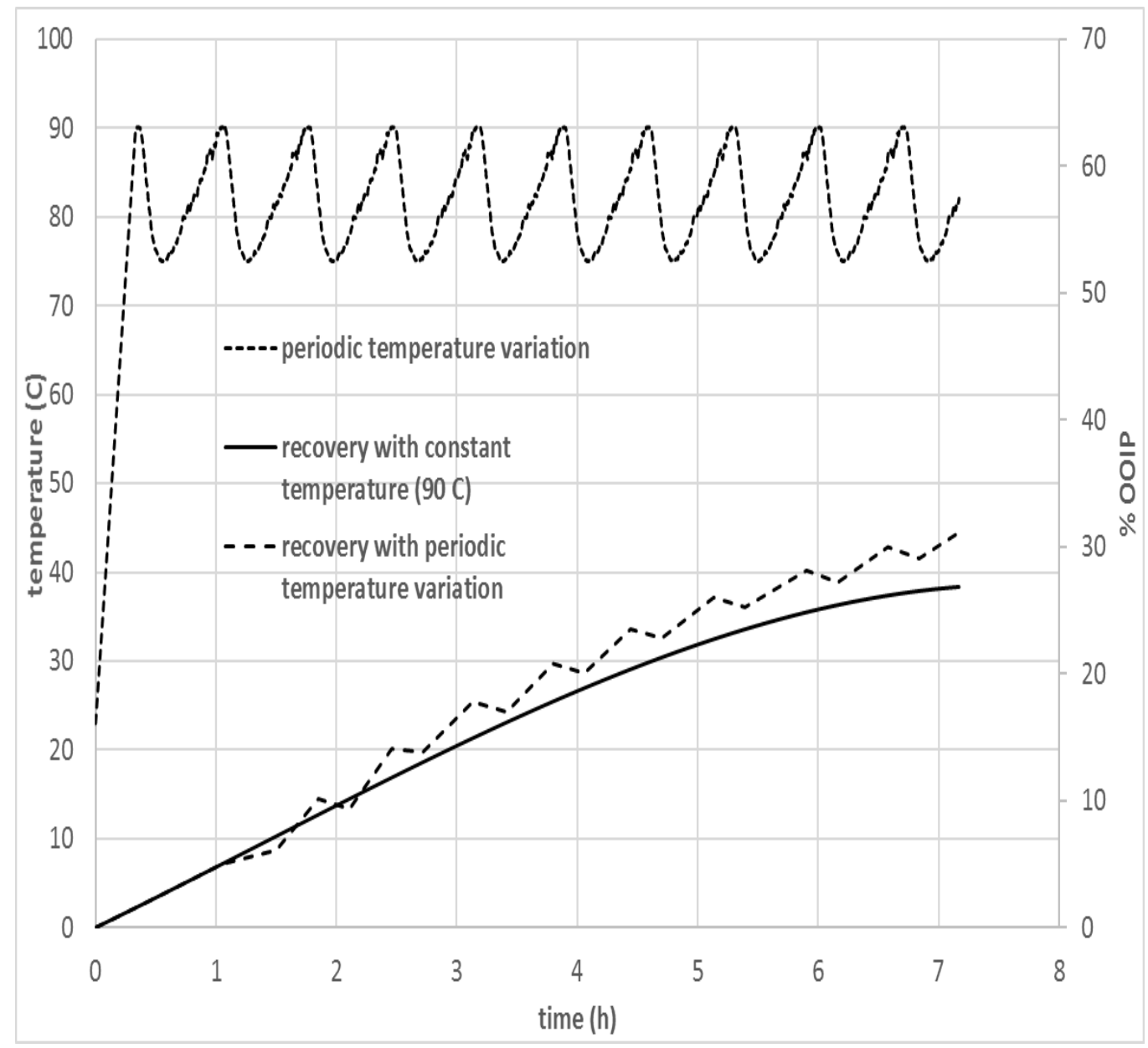

Figure 38: Oil recovery versus time at $0.514 \mathrm{MPa}$ absolute nitrogen pressure, constant $\left(90^{\circ} \mathrm{C}\right)$ as well as periodically varying air temperature in the range, $75-90^{\circ} \mathrm{C}$, and 87 Darcy model permeability 


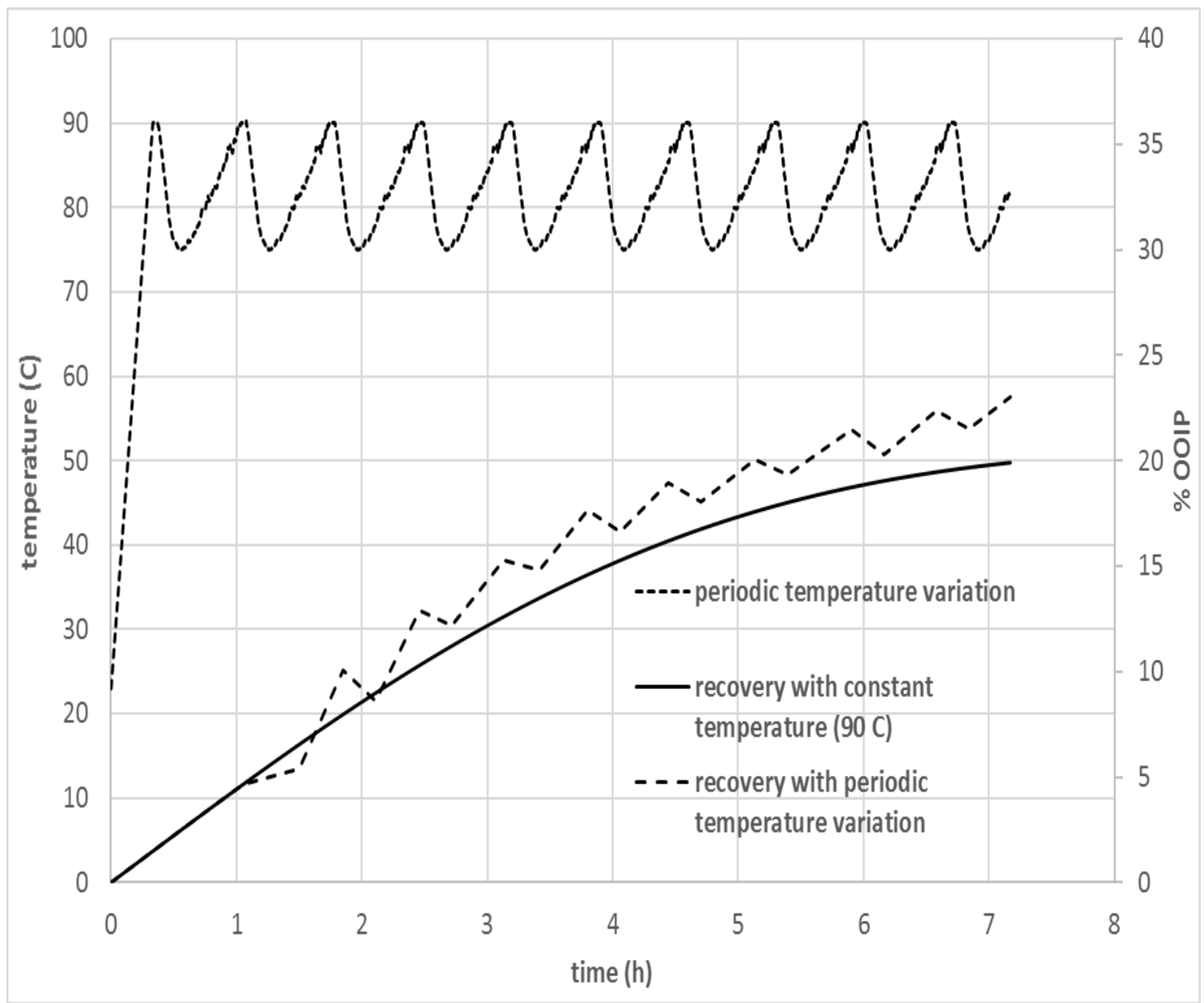

Figure 39: Oil recovery versus time at $0.514 \mathrm{MPa}$ absolute nitrogen pressure, constant $\left(90^{\circ} \mathrm{C}\right)$ as well as periodically varying air temperature in the range, $75-90^{\circ} \mathrm{C}$, and 40 Darcy model permeability

In summary, the above experimental results indicate that periodic temperature variation effectively enhanced oil recovery in comparison to the constant temperature of $90^{\circ} \mathrm{C}$ for both nitrogen and atmospheric air injection. Furthermore, the performance of nitrogen injection is marginally higher than air injection for heavy oil recovery. However, atmospheric air is free to access while capture and storage of nitrogen from the air is not cheap. 


\section{Appendix D: Dispersion Coefficient Calculation for Nitrogen Injection}

The initial and optimal $D(\omega, T)$ of nitrogen injection is plotted in Figure 40 at the injection pressure of $0.514 \mathrm{MPa}$, the physical model permeability of 204 Darcy, and different temperature of $25^{\circ} \mathrm{C}$, $50^{\circ} \mathrm{C}, 75^{\circ} \mathrm{C}$, and $90^{\circ} \mathrm{C}$. Similar to Figure 19 , the value of $[D(\omega, T)]$ respect to $25^{\circ} \mathrm{C}, 50^{\circ} \mathrm{C}, 75^{\circ} \mathrm{C}$, and $90^{\circ} \mathrm{C}$ increase to a maximum value, and then drops toward the end. Moreover, under the set operational conditions, the objective functional decreased monotonically to the minimum as shown in Figure 41. The maximum value of nitrogen dispersion coefficient is $2.556 \times 10^{-5}\left(\mathrm{~m}^{2} / \mathrm{s}\right)$ which is marginally higher than air dispersion coefficient.

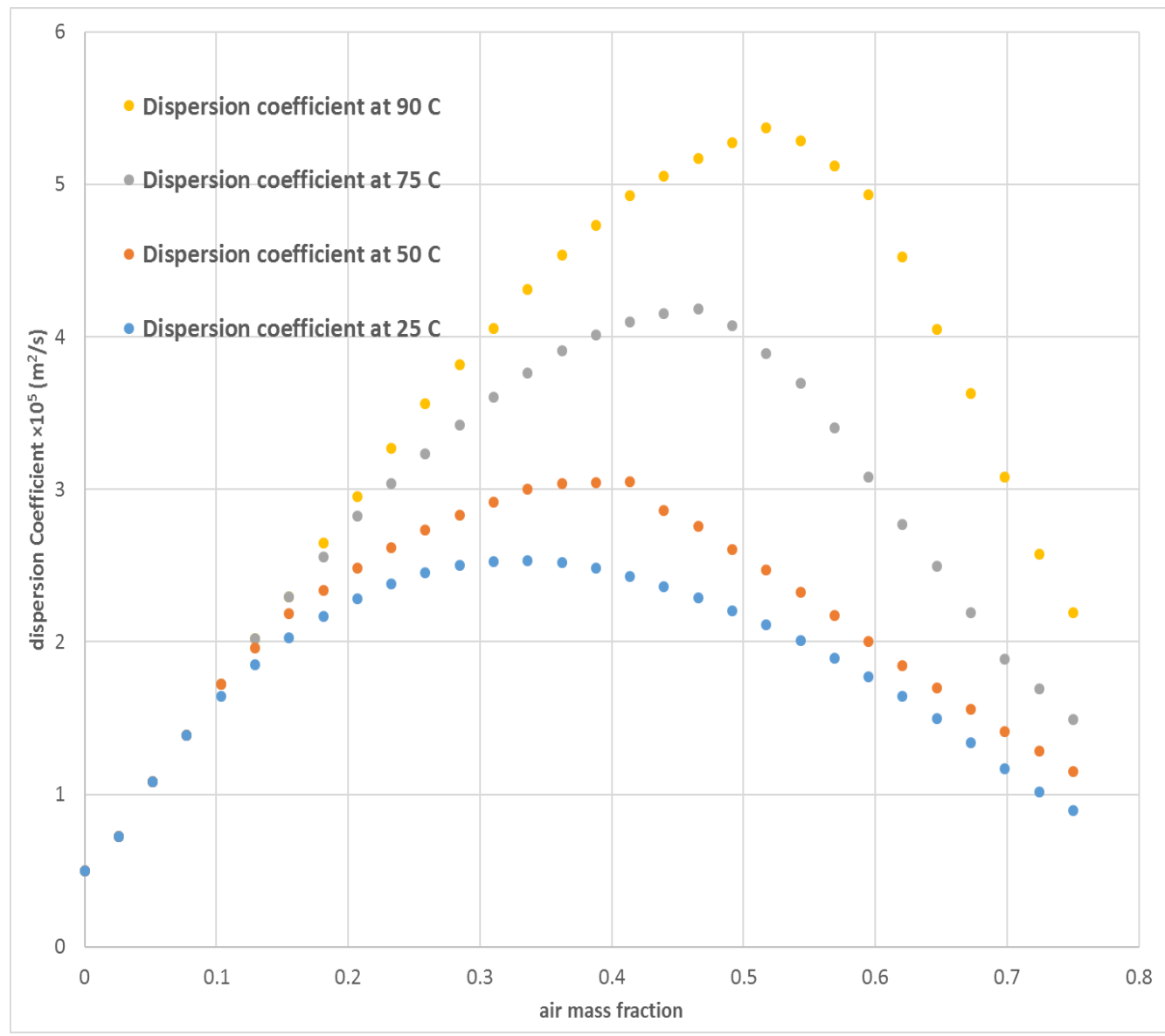

Figure 40: Dispersion coefficient function of nitrogen in heavy oil at different temperatures $\left(25^{\circ} \mathrm{C}\right.$, $50^{\circ} \mathrm{C}, 75^{\circ} \mathrm{C}$, and $90^{\circ} \mathrm{C}$ ) (Pressure 0.514 MPa; Permeability 204 Darcy) 


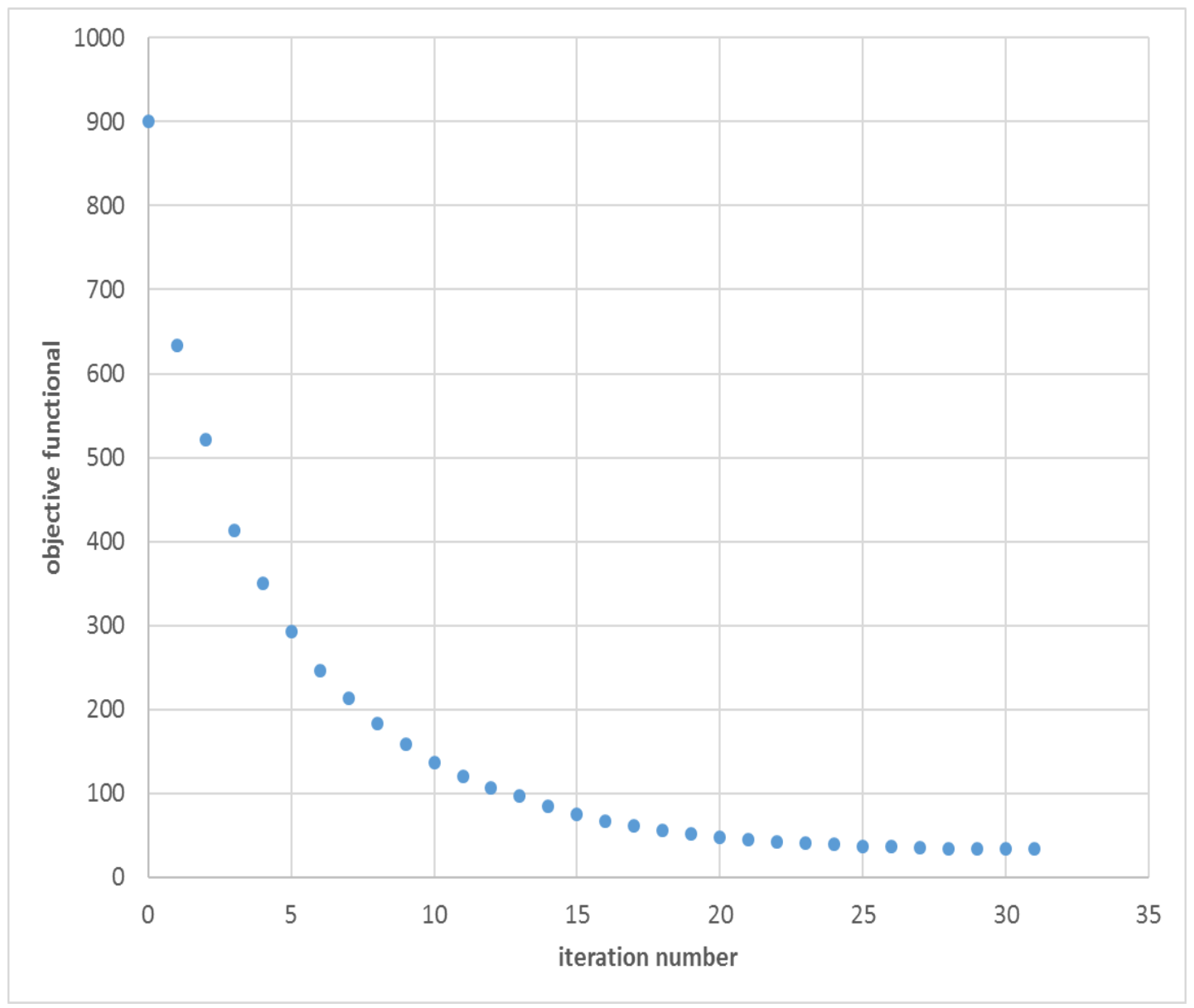

Figure 41: Nitrogen objective functional versus iteration number (Pressure $0.514 \mathrm{MPa}$; Permeability 204 Darcy) 


\section{Appendix E: Optimal Control Policy for Nitrogen Interfacial Temperature}

Figure 42 shows the nitrogen interfacial temperature versus time policy for different iteration based on an initial guess of a constant interfacial temperature of $90^{\circ} \mathrm{C}$ at the pressure of $0.514 \mathrm{MPa}$ and physical media permeability of 204 Darcy for 165 minutes of operational time. It resulted in an iterative increasing of the objective functional accompanied to the maximum improvement in $\left[T_{\text {int }}(t)\right]$. The objective functional increased monotonically to the highest value of 181.12 (Figure 43).

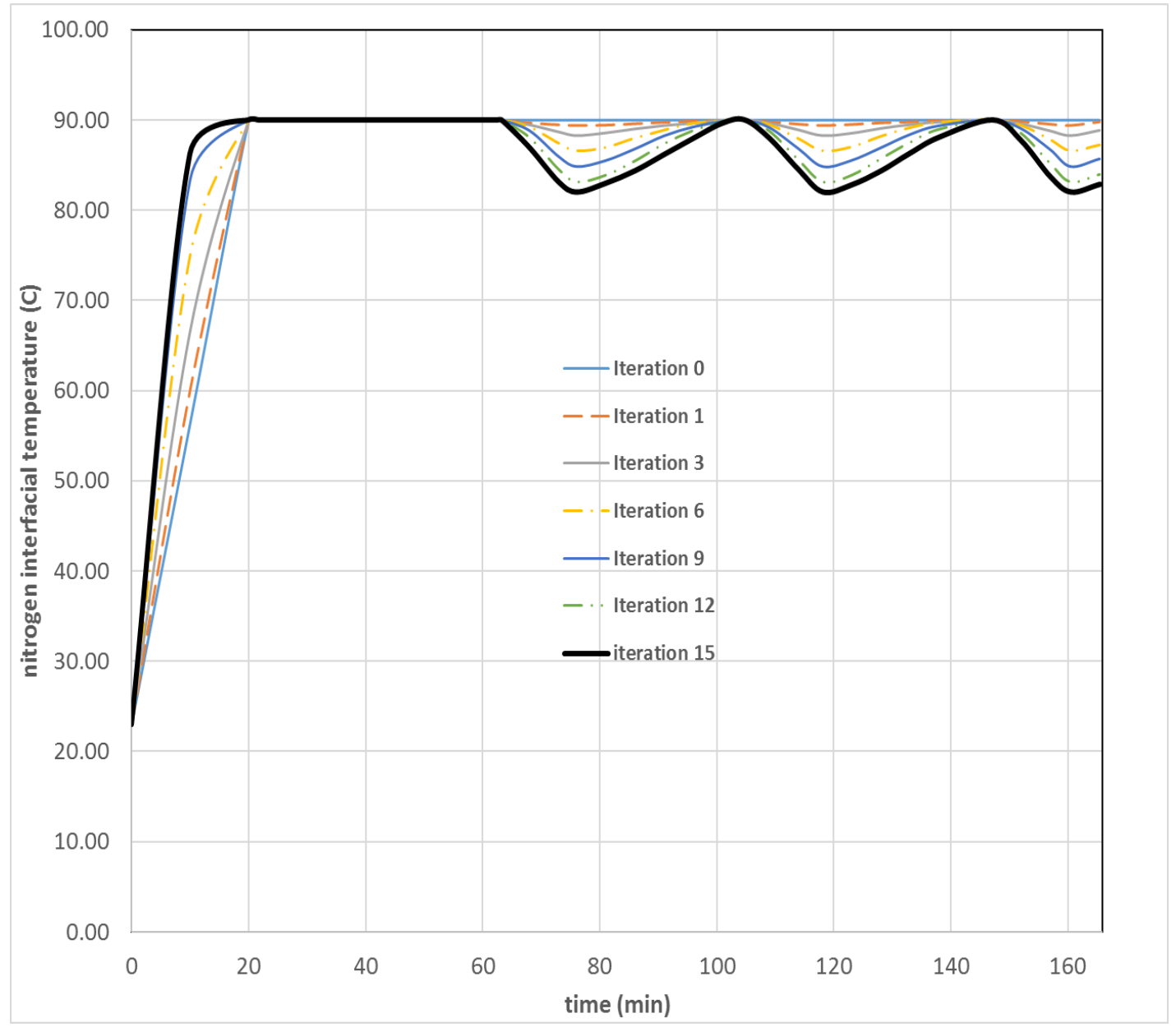

Figure 42: The nitrogen interfacial temperature $T_{\mathrm{int}}(t)$ at different iterations of the optimal control algorithm under an initial guess of the constant temperature of $90^{\circ} \mathrm{C}$ 


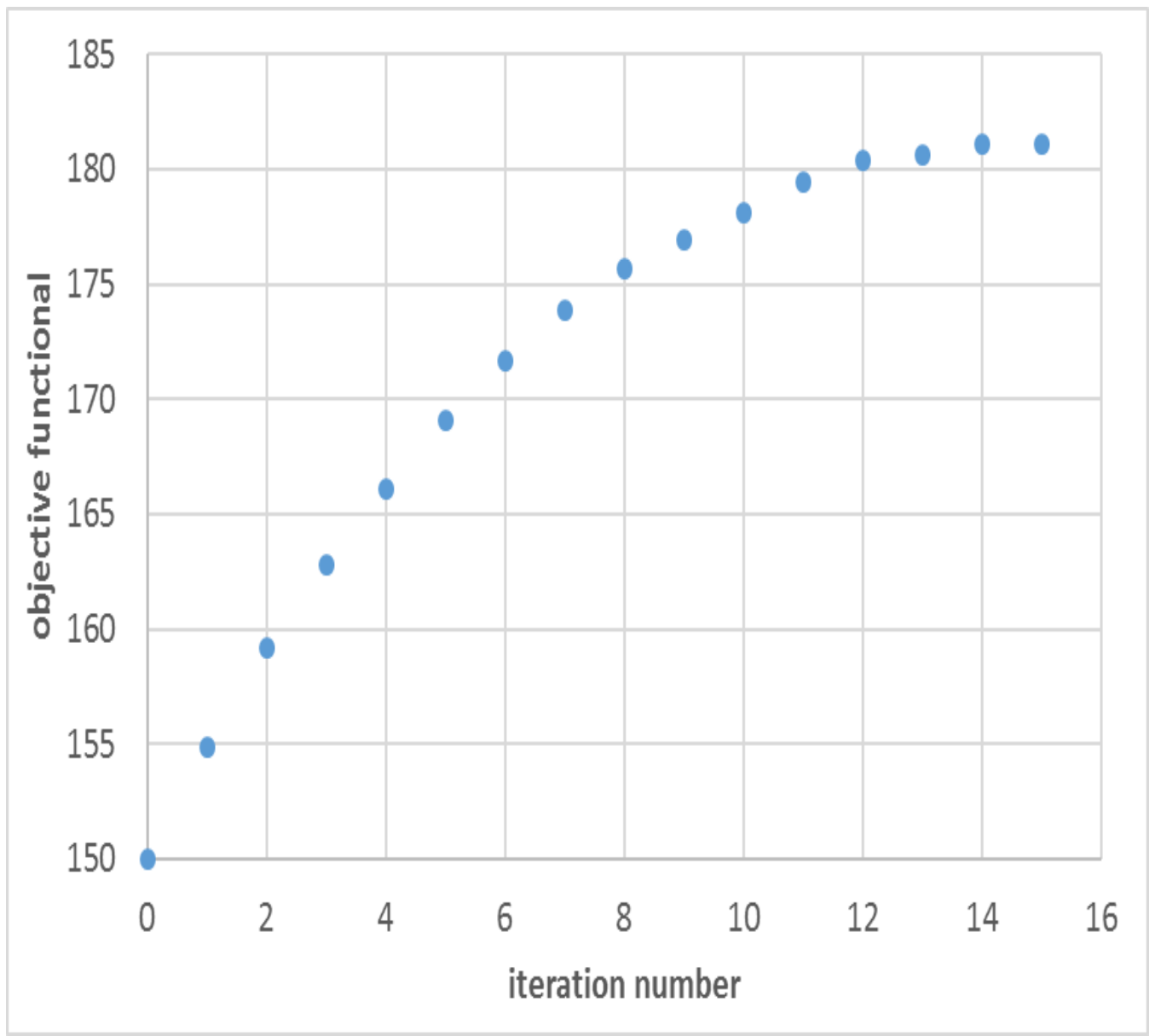

Figure 43: Nitrogen objective functional versus iteration number (204 Darcy and Pressure 0.514 $\mathrm{MPa})$ 


\section{$\underline{\text { Validation of Optimal Control Policy for Nitrogen Injection }}$}

Figure 44 shows that the experimental and calculated heavy oil recovery agree very well under the determined optimal $\left[T_{\text {int }}(t)\right]$. Thus, the average of relative errors at all sample times in the mass of heavy oil recovered was $2.01 \%$. Furthermore, this figure shows the difference between the experimental optimal interfacial temperature and simulated interfacial temperature is very small with the average relative error less than $2 \%$. Therefore, the little relative error demonstrates that we were able to validate the optimal control policy for nitrogen injection. Lastly, according to the results obtained (Figure 43), the optimal $\left[T_{\text {int }}(t)\right]$ policy enhances the heavy oil recovery significantly, with resulting in heavy oil recovery by $20.73 \%$ in comparison to the constant temperature of $90^{\circ} \mathrm{C}$. 


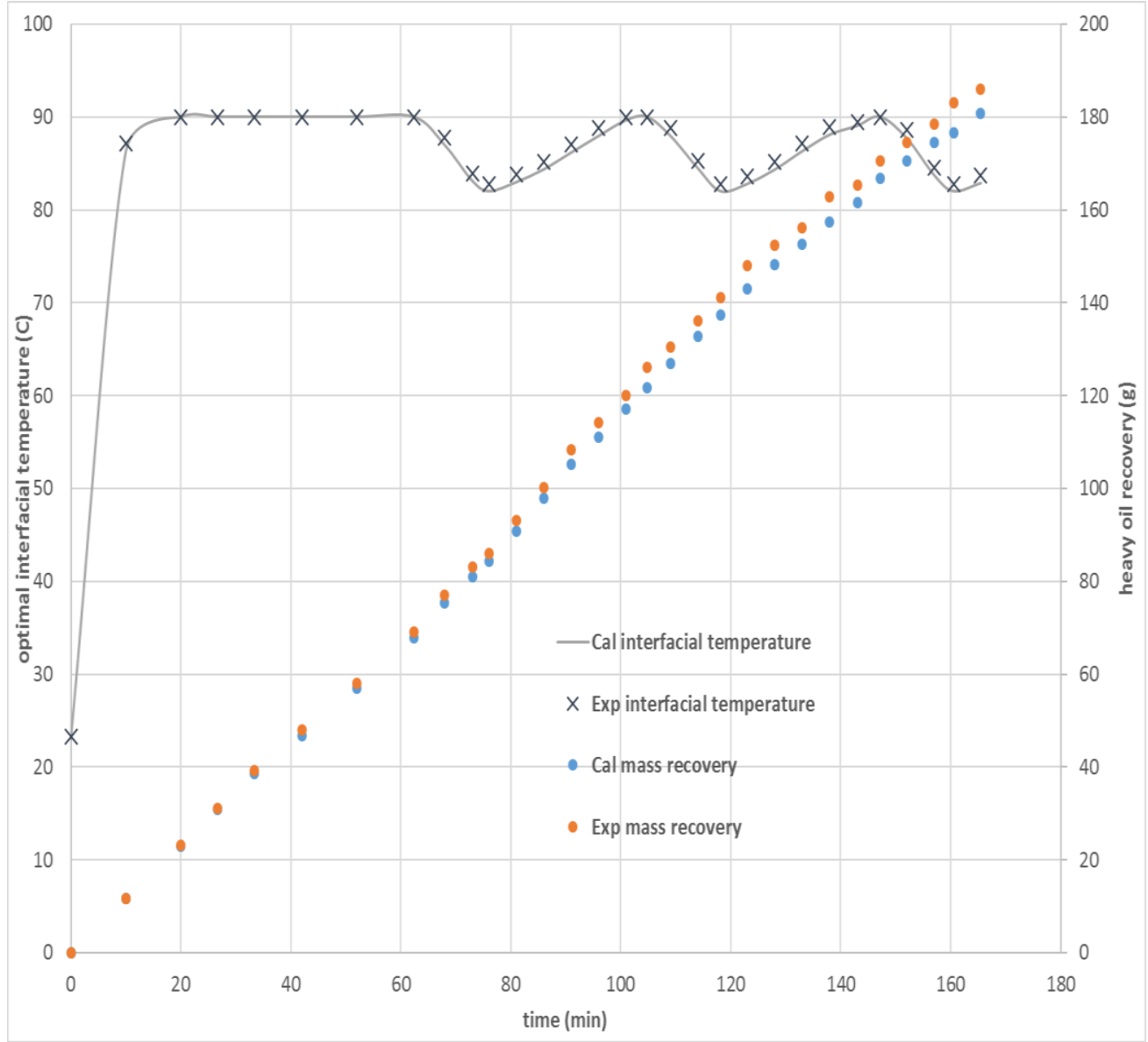

Figure 44: Experimental and calculated mass of live oil produced with time (Pressure 0.514 MPa, 204 Darcy, Solvent Nitrogen) 


\section{Appendix F: Permeability Effect on Optimal Policy for Nitrogen Injection}

Figures 45, 46, and 47 show the objective functional versus iteration number for nitrogen injection at 427, 87, and 40 Darcy, respectively.

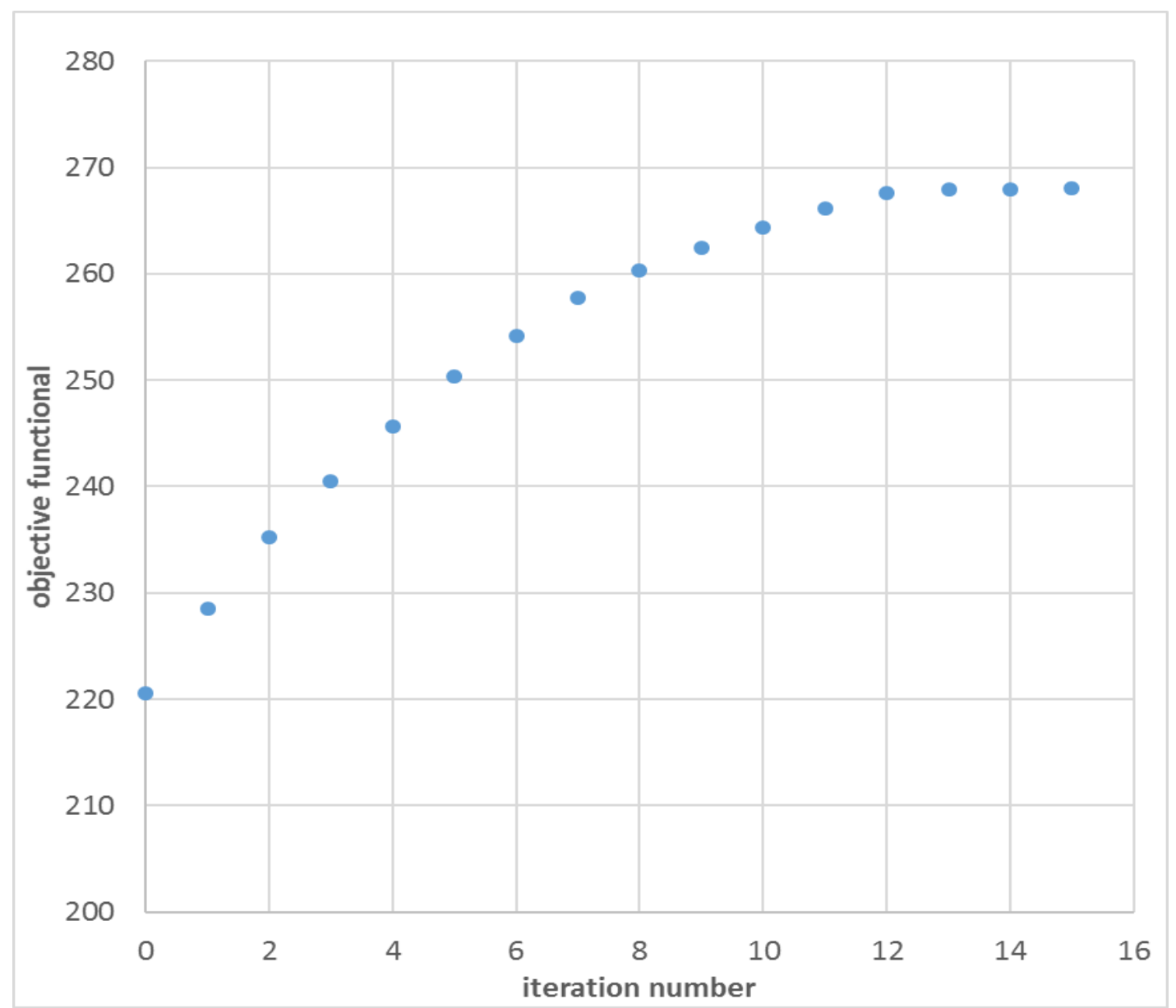

Figure 45: Nitrogen objective functional versus iteration number (427 Darcy and Pressure 0.514 $\mathrm{MPa})$ 


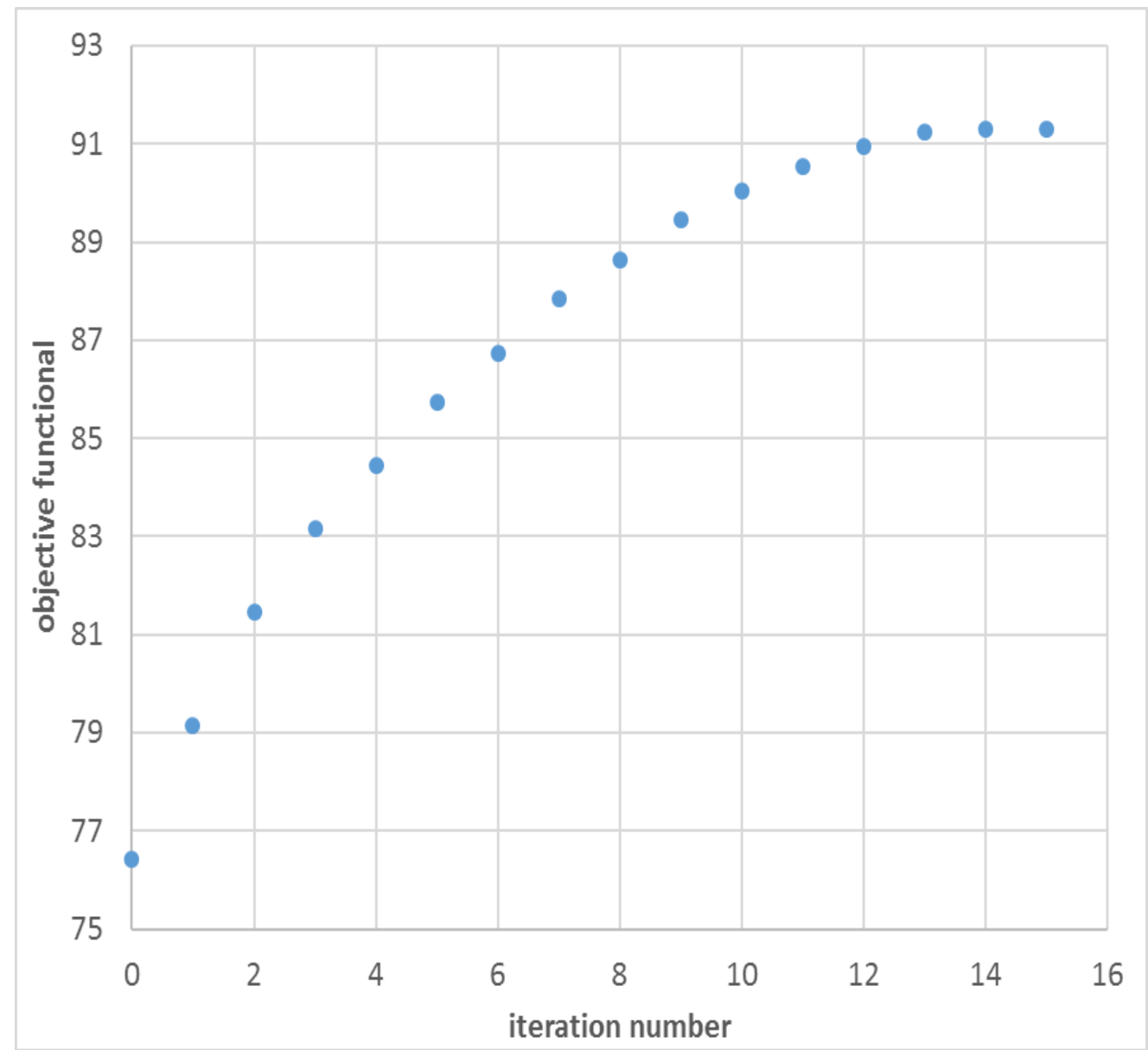

Figure 46: Nitrogen objective functional versus iteration number (87 Darcy and Pressure 0.514 $\mathrm{MPa})$ 


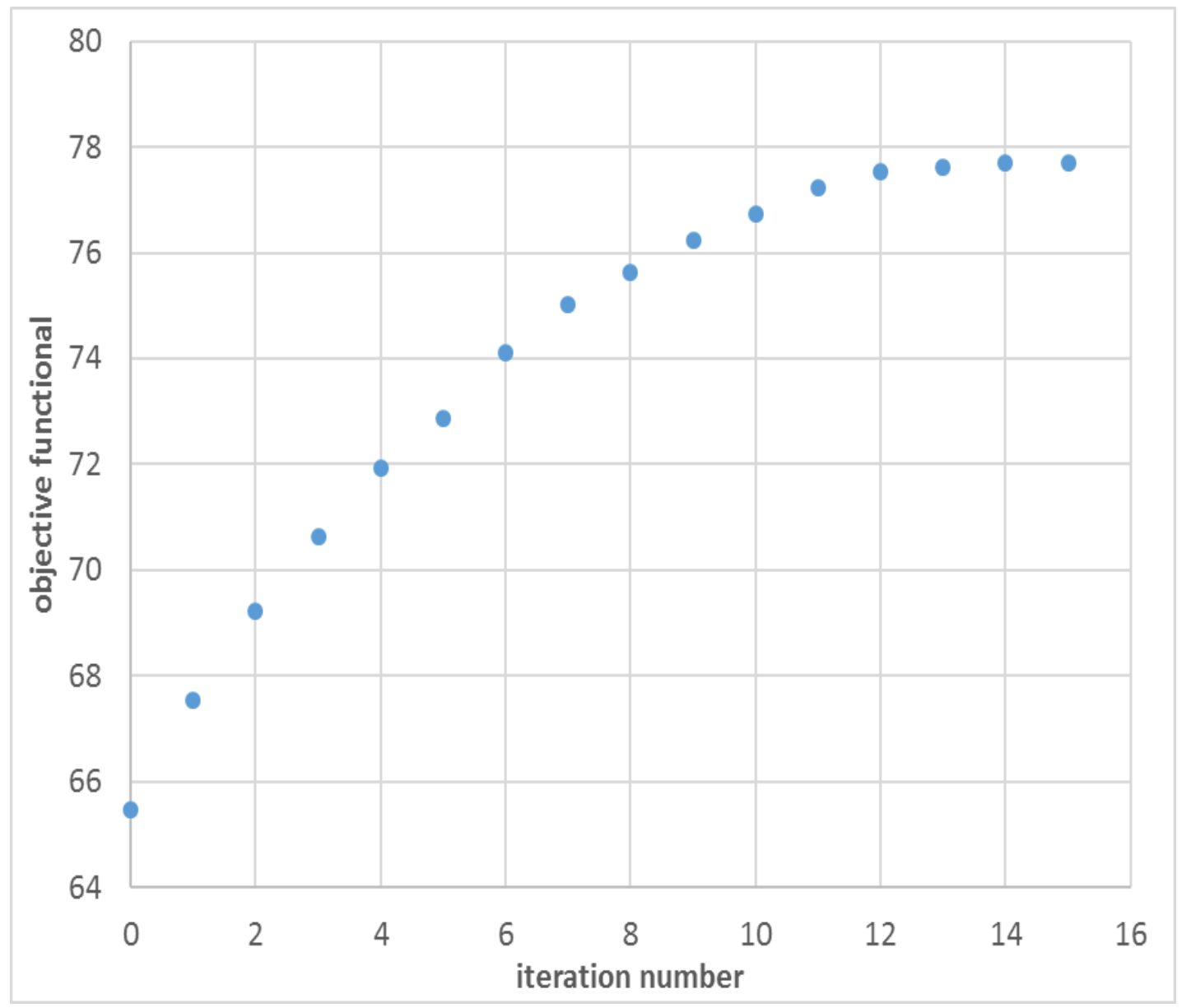

Figure 47: Nitrogen objective functional versus iteration number (40 Darcy and Pressure 0.514 $\mathrm{MPa})$ 
Validation of Optimal Control Policy for Nitrogen Injection at Different Permeability

Figure 48 shows the comparison between the calculated objective functional versus the experimental value at three different packs of permeability of 40, 87, and 427 Darcy. The average of relative errors for 40, 87, and 427 Darcy at all sample times in the mass of heavy oil recovered were found to be $2.2 \%, 3 \%$, and $3.9 \%$, respectively.

Thus, the calculated objective functional agree very well with experimental value at different permeability.

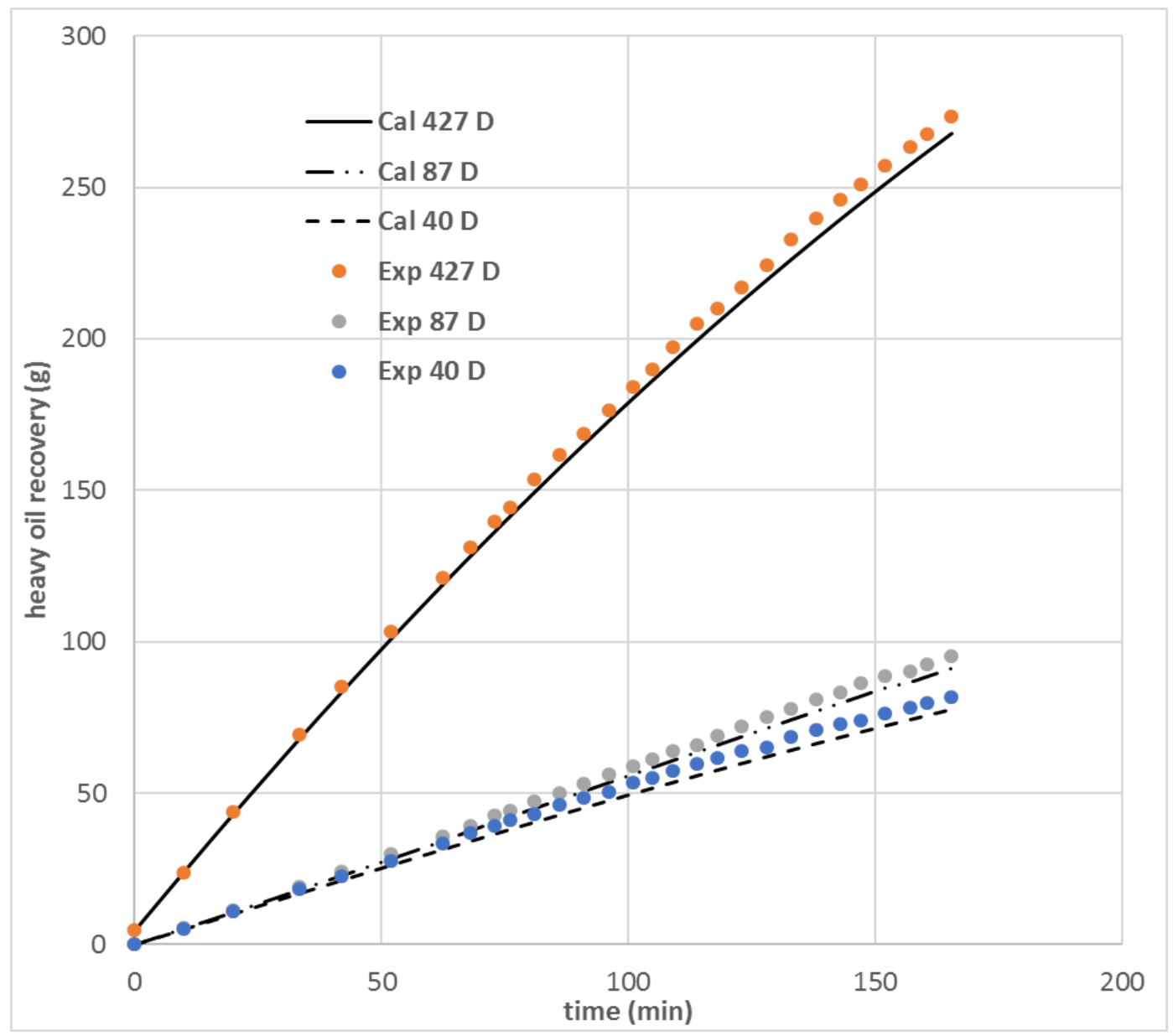

Figure 48: Experimental and calculated heavy oil recovery using Nitrogen at three different medium permeability 


\section{References}

1. Lyons, W.C.; Plisga, G.J. Standard Handbook of Petroleum and Natural Gas Engineering, 2th ed.; Elsevier, Amsterdam, Netherlands, 2005; pp 7-11.

2. National Energy Board, Canada's Oil Sands - Opportunities and Challenges to 2015: An Update. An Energy Market Assessment June 2006 Calgary; http://www.nebone.gc.ca/nrg/sttstc/crdlndptrlmprdct/rprt/archive/pprtntsndchllngs20152006/pprtntsndchl lngs20152006-eng.pdf, 2006; accessed in 22 October,2016; pp viii-x, 27-28.

3. Alvarado, V.; Manrique E. Enhanced Oil Recovery - Field Planning and Development Strategies; Gulf Professional Publishing, Texas, 2010; pp 7-11, 43-64, 133-153.

4. Head, I. M.; Jones, D. M., and Larter, S. R. Nature, 2003, 426, 344-352.

5. Larter, S.; Whilhelins, A.; Head, I; Koopmans, M; Aplin, A; Di Primio, R; Zwach, C; Erdmann, M; Telnaes, N; Org. Geochem, 2003, 34, 601-613.

6. Larter, S.; Huang, H.; Adams, J.; Bennett, B.; Jokanola, O.; Oldenburg, T.; Jones, M.; Head, I.; Riediger, C.; Fowler, M.; American Association of Petroleum Geologists Bulletin, 2006, 90, 921-938.

7. Meyer, R. F; Attanasi, E. D; Freeman, P. A; Heavy Oil and Natural Bitumen Resources in Geological Basins of the World. US Geological Survey, Virginia, 2007, Accessed March 22, 2017, http://pubs.usgs.gov/of/2007/ 1084/OF2007-1084v1.pdf

8. Speight, J.G. Enhanced Recovery Methods for Heavy Oil and Tar Sands; Gulf Publishing Company, Texas, 2009; pp 221-255.

9. Jayasekera, A. T.; Goodyear, S. G.; SPE Reservoir Evaluation \&amp; Engineering, 2000, 3, 371-379.

10. Shah A.; Fishwick, R.; Wood, J.; Leeke, G.; Rigby, S.; Greaves, M. Energy Environ. Sci., 2010, 3, 700-714

11. Banerjee, D.K. Oil Sands, Heavy Oil \& Bitumen - From Recovery to Refinery; PennWell, Oklahoma, 2012; pp 59-64.

12. Upreti, S.R.; Lohi, A.; Kapadia, R.A.; El-Haj, R. Energ. Fuel.2007, 21, 1562-1574

13. In Encyclopedia of Physical Science and Technology 3rd Edition, vol. 18. Robert A. Meyers Ed., Academic Press, 2001, pp 503-518 
14. Stosur, G.J. EOR: Past, Present and What the Next 25 Years May Bring; Proceedings of the Society of Petroleum Engineers (SPE); Kuala Lumpur, Malaysia, October 20-2, 2003 (SPE 84864).

15. Abu El Ela, M.; Samir, M.; Sayyouh, H.; El Tayeb, S. Oil. Gas. J.2008, 106, 40-45.

16. Satter, A.; Iqbal, G.M.; Buchwalter, J.L.; Practical Enhanced Reservoir Engineering: Assisted with Simulation Software. PennWell, Oklahoma, 2008, pp 549-566

17. Li, W.; Mamora, D.D. Phase Behavior of Steam with Solvent Co-injection under Steam Assisted Gravity Drainage (SAGD) Process; SPE EUROPEC/EAGE Annual Conference and Exhibition, Barcelona, Spain 14-17 June, 2010 (SPE 130807).

18. Alvarez, J.; Han, S.; Journal of Petroleum Science Research. 2013, 2, 116-127.

19. Upreti, S.R.; Mehrotra, A.K. Can. J. Chem. Eng.2002, 80, 116-125.

20. Manrique, E.J.; Thomas, C.P.; Ravikiran, R.; Kamouei, M.I.; Lantz, M.; Romero, J.L.; Alvarado, V. EOR: Current Status and Opportunities; SPE Improved Oil Recovery Symposium, Oklahoma, USA 24-28 April, 2010 (SPE 130113).

21. Dooley, J.J.; Dahowski, R.T.; Davidson, C.L. CO2-driven Enhanced Oil Recovery as a Stepping Stone to What?; U.S. Department of Energy, http://www.pnl.gov/main/publications/external/technical_reports/PNNL-19557.pdf, 2010; accessed in 12 February, 2014; pp 2-27.

22. Alvarado, V.; Manrique, E. Energies. 2010, 3, 1529-1575.

23. Moritis, G. Oil. Gas. J.2008, 106, 41-42, 44-59.

24. Huang, T.; Zhou, X.; Yang, H.; Liao, G.; Zeng, F. Petroleum. 2017, 3, 68-78

25. Telmadarreie, A., Evaluating the Potential of $\mathrm{CO} 2$ Foam and CO2 Polymer Enhanced Foam for Heavy Oil Recovery in Fractured Reservoirs: Pore-Scale and Core-Scale Studies. PhD's thesis, University of Alberta, Alberta, Canada. 2017, pp 7-17, 36-39

26. Manrique, E.J.; Muci, V.E.; Gurfinkel, M.E. Reserv. Eval. Eng.2007, 10, 667-686.

27. Moritis, G. Oil. Gas. J.2001, 99, 68-73.

28. Muggeridge, A.; Cockin, A.; Webb, K.; Frampton, H.; Collins, I.; Moulds, T.; Salino, P. Phil. Trans. R. Soc. A.2014, 372, 20120320.

29. Rafiq Islam, M.; Chhetri, A.B.; Khan, M.M. Greening of Petroleum Operations: The Science of Sustainable Energy Production. Wiley-Scrivener Publishing LLC, Massachusetts, 2010, pp 577-633 
30. Gurgel, A.; Moura, M.C.P.A.; Dantas, T.N.C.; Barros Neto, E.L.; Dantas Neto. A.A.; Brazilian Journal of Petroleum and Gas. 2008, 2, 83-95.

31. Sheng, J., Modern Chemical Enhanced Oil Recovery - Theory and Practice. Elsevier, Amsterdam, 2011, pp 101-206, 239-335, 501-567

32. Sandersen, S.B., Enhanced Oil Recovery with Surfactant Flooding. PhD's thesis, Technical University of Denmark, Copenhagen, Denmark. 2012, pp 6-20

33. Lazar, I.; Petrisor, I.G.; Yen, T.F.; Petrol. Sci. Technol. 2007, 25, 1353-1366.

34. Fan, C.; Zan, C.; Zhang, Q.; Shi, L.; Hao, Q.; Jiang, H.; Wei, F. Ind. Eng. Chem. Res.2015, $54,6634-6640$.

35. Wang, C.; Liu, H.; Pang, Z.; Wang, J.; Chen, C.; Wang, C.; Wu, Z. Energy Fuels. 2015, $29,6242-6249$.

36. Butler, R. M.; Mokrys, I. J. J. Can. Pet. Technol. 1991, 30, 97-106

37. Das, S. K.; Butler, R. M. J. Pet. Sci. Eng. 1998, 21, 43-59

38. Kapadia, R.A. Dispersion Determination in Vapex: Experimental Design, Modeling and Simulation. Master's thesis, Ryerson University, Toronto, Ontario, Canada, 2004, pp 1126

39. Lederer, E. L. Proceedings of World Petroleum Congress, South Kensington, London, July 19-25, 1933; Dunstan, A. E., Sell, G., Eds.; Offices of the Congress: London, 1934.

40. Das, S. K.; Butler, R. M. Can. J. Chem. Eng. 1996, 74, 985-992

41. Jin, W. Heavy Oil Recovery Using the Vapex Process. Master's thesis, University of Waterloo, Waterloo, Ontario, Canada, 1999, pp 11-26

42. Upreti, S.R.; Mehrotra, A.K. Ind. Eng. Chem. Res.2000, 39, 1080-1087

43. Hayduk, W.; Cheng, S. C. Chem. Eng. Sci. 1971, 26, 635-646

44. Perkins, T. K.; Johnston, O. C. SPE J. 1963, 3, 70-84

45. Pal, K.; Joyce, M.K.; Fleming, P.D., TAPPI JOURNAL. 2006, 9, 1-3

46. Ediriweera, M.P.; Halvorsen, B.M., A Study of the Effect of Relative Permeability and Residual Oil Saturation on Oil Recovery; Proceedings of the $56^{\text {th }}$ SIMS, Linkoping, Sweden, October 07-09, 2015

47. Kirk, D., Optimal Control Theory: An Introduction, Courier Corporation, 2004, 167-177

48. Upreti, S. R., Optimal Control for Chemical Engineers, 1 edition, Taylor and Francis Group, FL, USA, CRC Press, 2012, pp 57-85, 185-231, 235-265 
49. Abukhalifeh, H. Determination of Concentration-dependent Dispersion of Propane in Vapor Extraction of Heavy Oil; PhD thesis, Ryerson University, Canada, 2010, pp 89-95, 98-104

50. Muhamad, H. Optimal Control of Vapor Extraction of Heavy Oil; $\mathrm{PhD}$ thesis, Ryerson University, Canada, 2012, pp 33-34, 59-86, 94-97

51. El-Haj, R. Experimental Determination of Solvent Gas Dispersion in Vapex Process; MASc Thesis, Ryerson University, Canada, 2007, pp 28-35, 56-68

52. Oduntan, A.R. Heavy Oil Recovery Using the Vapex Process: Scale Up and Mass Transfer Issues. Master's Thesis, University of Waterloo, Waterloo, Canada, 2001, pp 12-30

53. Muhamad, H.; Upreti, S. R.; Lohi, A.; Doan, H. Energy \& Fuels.2012, 26, 3514-3520

54. Moghadam, S.; Nobakht, M.; Gu, Y. J. Pet. Sci. \& Eng.2009, 65, 93-104

55. Hepler, L.G.; Hsi, C. AOSTRA Technical Publication Series \#6: Technical Handbook on Oil Sands; Bitumens and Heavy Oils, Alberta Oil Sands Technology and Research Authority, Alberta 1989; pp 281-300.

56. Ajumobi, A. A Study on the Heavy Crude Oil Viscosity Reduction with the Dissolution of Nitrogen and Carbon Dioxide; MASc Thesis, The City College of New York, U.S., 2015, pp 18, 27-31, 36-37.

57. El-Haj, R.; Lohi, A.; Upreti, S.R. J. Pet. Sci. Technol.2009, 67, 41-47.

58. Mailybaev, A.A.; Bruining, J.; Marchesin, D. Combustion and Flame 2011, 158, 10971108.

59. Khoshnevis Gargar, N. Combustion for Enhanced Recovery of Light Oil at Medium Pressures. MASc Thesis, University of Calgary, Canada, 2014, pp 71-90.

60. Galas, C.; Clements, A.; Jaafar, E.; Jeje, O.; Holst, D.; Holst, R. Identification of Enhanced Oil Recovery Potential in Alberta phase 2 Final Reportfor Energy Resources Conservation Board. http://www.ags.gov.ab.ca/publications/pdf_downloads/ercb-eor-report2.pdf, 2012; accessed in 14 October, 2015; pp 2-14.

61. Imran, M. Dispersion Coefficient Determination in Vapex Process. MASc Thesis, Ryerson University, Canada, 2008, pp 56-65. 